\title{
The Feasibility of Establishing a Morphological Cell Profiling Assay to Assess the Bioactivity of Natural Products
}

\author{
By
}

Lauren Taylor

A thesis submitted to Victoria University of Wellington - Te Herenga Waka in fulfilment of the requirements for the degree of Master of Biomedical Science

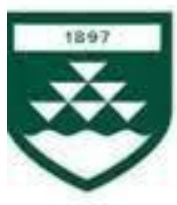

VICTORIA UNIVERSITY OF

WELLINGTON

TE HERENGA WAKA

June 2021 


\section{Acknowledgements}

Completing a Masters degree has been an "unprecedented" time, and that's without the global pandemic on top of it. Without trying to sound cliché, the work that has gone into this thesis would not have been possible without my supervisors, my lab colleagues, my family and friends. You have all supported me in such different ways that have collectively made a massive difference to not only this body of work, but also my wellbeing, so thank you.

To Melanie McConnell, a monstrous thank you must obviously go to you. You are an incredibly intelligent and understanding person with tonnes of spunk, and because of that, as well as your unfaltering supervision, the past two and a half incredibly hard years have been filled with far less stress and more happiness than they could have.

To Rob Keyzers, a huge thank you must also go to you. Your support and chemistry knowledge over this project and the trust you have put in me over the past few years has been quite frankly, unbelievable. I cannot wait to continue working with you for the duration of my doctorate.

To the MMc lab members, Leticia Castro, Matthew Rowe, Georgia Carson, Rebecca Dawson, Anna Tribe, Saskia Ymker, Brittany Lewer, Devlin Forsythe, Helena Abolins-Thompson, Elena Orsman, Danielle Lewthwaite, Huri Bakhshi and Nico Lieffering thank you for your support and knowledge, and putting up with me and my chaotic ways in the lab and office.

To the RAK lab members, Joe Bracegirdle, Sarah Andressand, Helen Woolner, Jonathan Singh, Natascha Lewe, Manu Blank, Jennie Ramirez and Kate Greer, you instructions have been invaluable for the past four years and I would have been lost without you.

To the Wellington Microscopy Group, especially Alfonso Schmidt from Malaghan Institute for Medical Research, Lee Botes from Olympus, as well as Kendra Boyes and Mike Fairhurst.

To my family, thank you for being my escape place, my financial aid, and my rocks throughout such a hectic time. And naturally thanks Felix for all the cuddles.

To my friends, Jack, Alice, Danielle, Emma, Caitlin, Kaileen and Elaina; thank you for the constant emotional support and being an unwavering source of comfort and laughter.

And lastly, and definitely the least, thank you to 2020 for all the learning opportunities. \#gratefulforthegrowth 


\section{Abstract}

Morphological cell profiling (MCP) is an assay which quantifies the morphology of cells and cellular components. Changes in the morphology of cells following compound treatment has been shown to assess the bioactivity, and even propose a target of said compound. It is a powerful assay that can be used to assess novel compounds for drug candidature. However, it is currently not transferable between institutions. Thus, this project evaluated the feasibility of establishing an MCP assay at Victoria University of Wellington - Te Herenga Waka to assess the bioactivity of novel natural products from the natural products laboratory.

First, a new individualistic approach to select compound concentration for MCP was assessed by flow cytometry with six exemplary drugs. Results concluded this flow cytometry approach can realise an ideal concentration for individual compounds in which the compound treatment was bioactive but not cytotoxic. This approach provides more biologically relevant and accurate information about a compound's bioactivity than previous MCP methods.

Second, the feasibility of establishing the assay was assessed by testing the same six drugs through protocol common to MCP assays. Various stains and microscopes were tested for suitability for an MCP; image quantitative software CellProfiler was evaluated for MCP analysis ease; and quality control protocol was attempted. Ultimately, an MCP is currently not feasible at Te Herenga Waka as multiple components to establish the assay are too difficult and time intensive to complete.

Third, discorhabdin E, a pyrroloiminoquinone alkaloid, was isolated from the New Zealand marine sponge, Latrunculia kaakaariki. The compound was tested as if it were a novel natural product compound through the new flow cytometry concentration selection protocol to test its effectivity, and was subsequently assessed by morphological analysis. 


\section{Table of Contents}

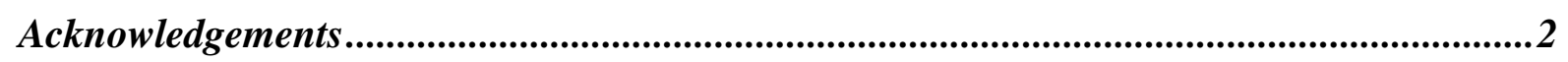

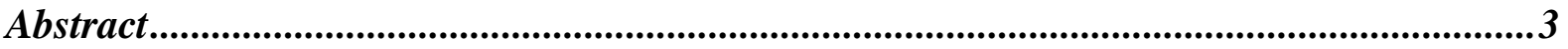

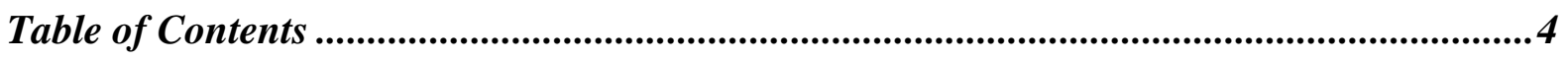

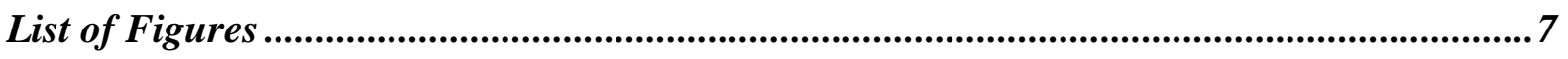

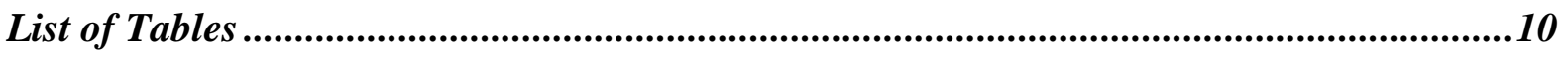

List of Abbreviations .....................................................................................................................11

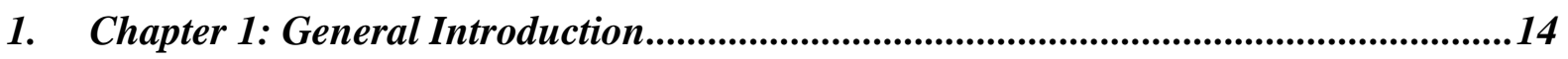

1.1. Drug Discovery …..................................................................................................................................15

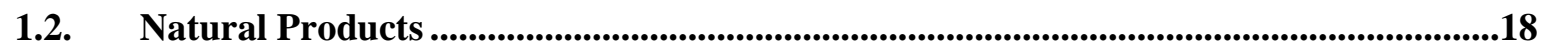

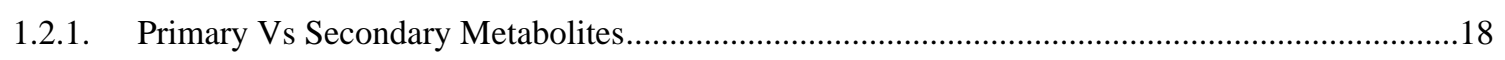

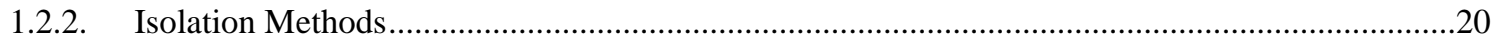

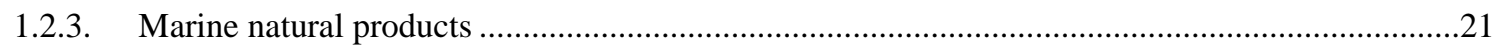

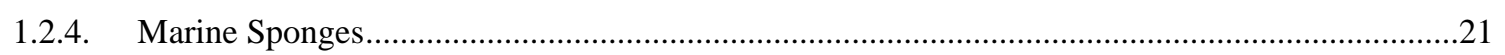

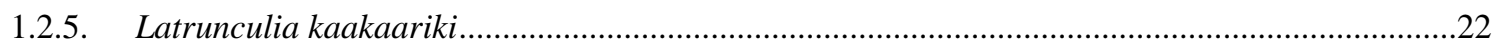

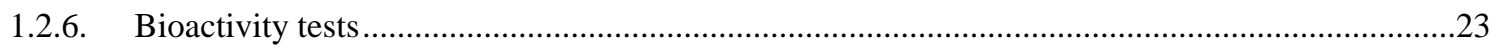

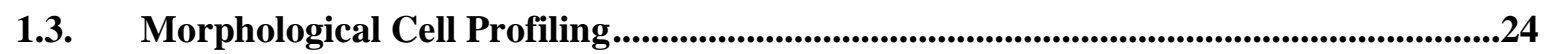

1.3.1. Morphological Cell Profiling assay development..................................................................24

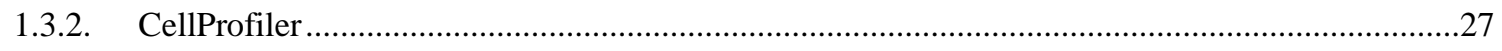

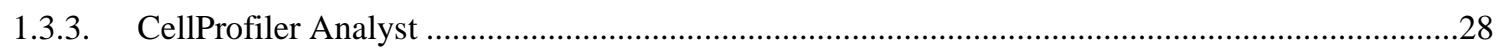

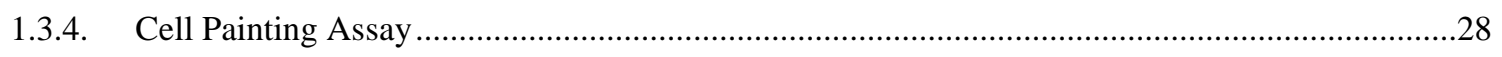

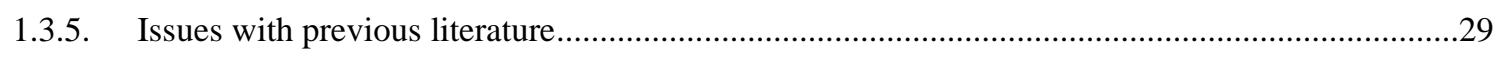

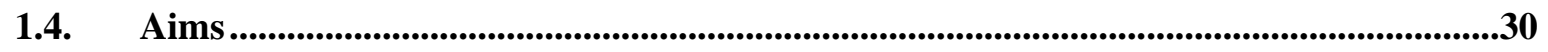

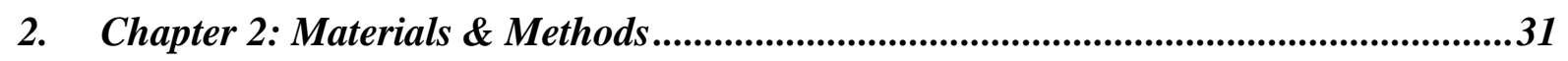

2.1. Biology ….......................................................................................................................................32

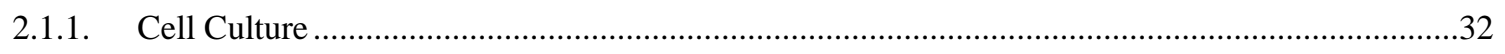

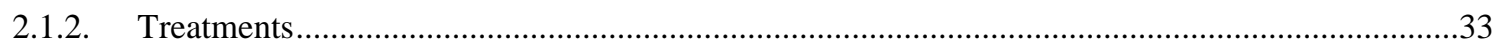

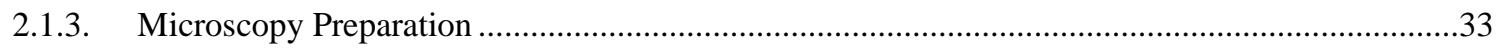

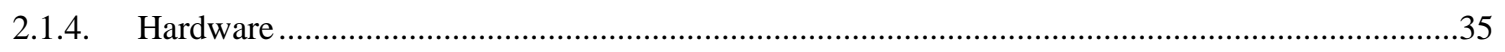

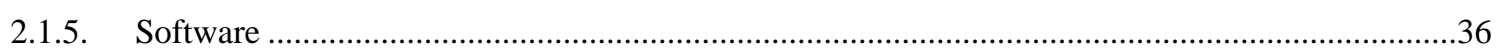




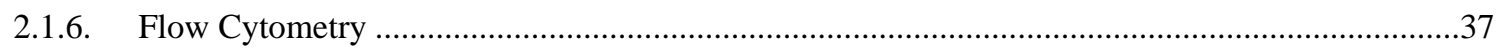

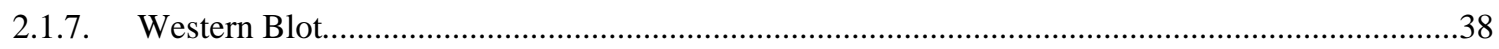

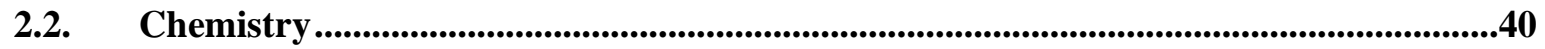

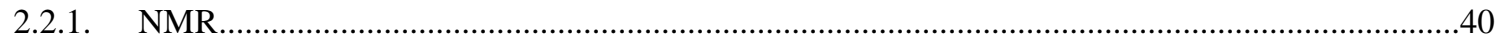

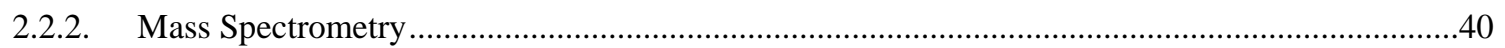

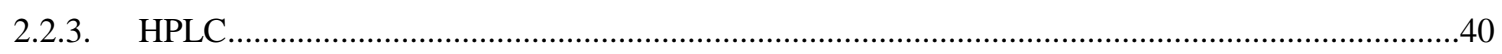

2.2.4. Benchtop Column Chromatography ….......................................................................... 41

2.2.5. Isolation of pyrroloiminoquinones from Latrunculia kaakaariki ..............................................41

3. Chapter 3: Development of a flow cytometry-based method to determine compound concentration for a MCP assay ..................................................................................................43

3.1. Introduction ...................................................................................................................................44

3.2. Aims ..................................................................................................................................45

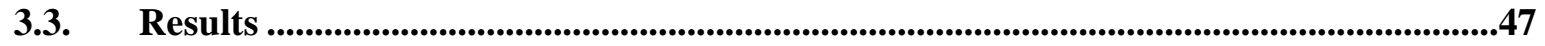

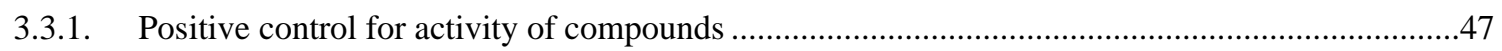

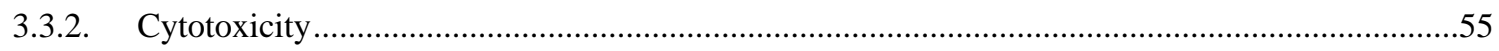

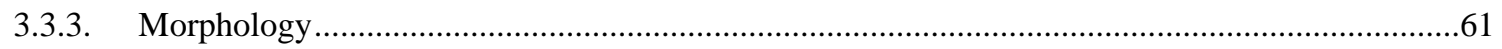

3.4. Discussion ........................................................................................................67

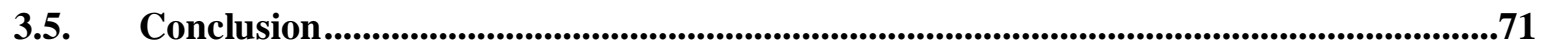

4. Chapter 4: Establishing the foundations of an imaged-based MCP at Te Herenga

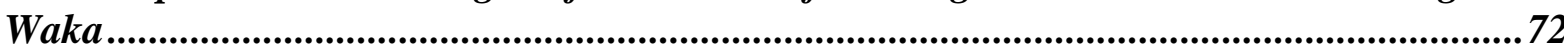

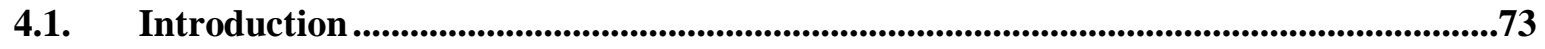

4.2. Aims ........................................................................................................

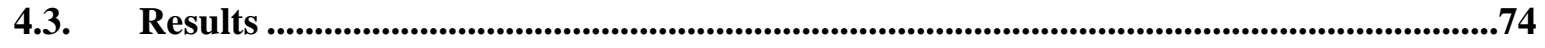

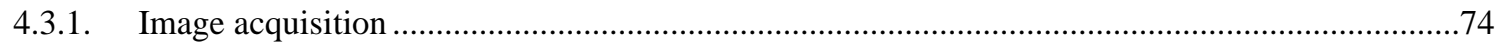

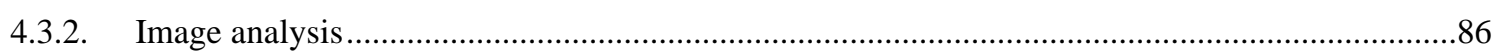

4.4. Discussion ....................................................................................................................................95

4.5. Conclusion .....................................................................................................................................97

5. Chapter 5: Isolate compounds from Latrunculia kaakaariki to assess if the protocol established in this project is fit for purpose ..............................................................................98

5.1. Introduction ............................................................................................................................99

5.2. Aims ................................................................................................................................99

5.3. Results …….................................................................................................................................100 


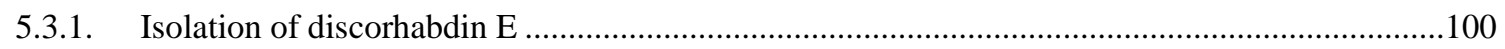

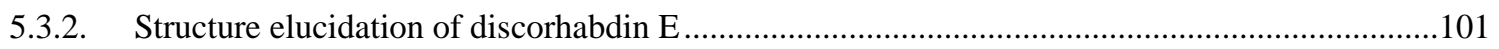

5.3.3. Discussion to confirm discorhabdin E, not discorhabdin G................................................104

5.3.4. Bioactivity assessment of discorhabdin E by flow cytometry ............................................108

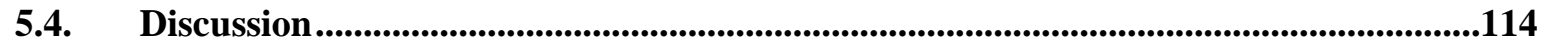

5.5. Conclusion.........................................................................................................115

6. Chapter 6: Final Discussion .......................................................................116

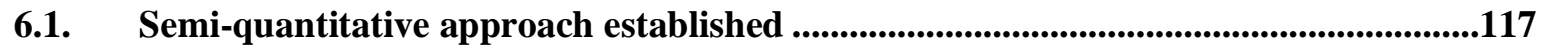

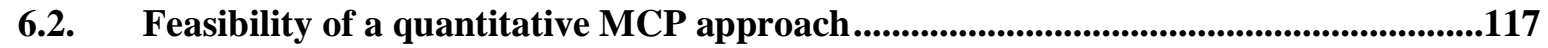

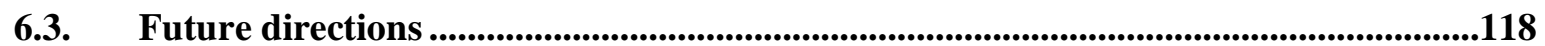

6.4. Concluding remarks......................................................................................................119

Appendix..........................................................................................................................120

References ............................................................................................................148 


\section{List of Figures}

Figure 1: A schematic of the drug discovery and development pipeline. Examples of some of the procedures for each step are noted. Adapted from Mohs \& Greig (2017).

Figure 2: Phase Success of academic drug discovery and development between 1991 and 2010. Adapted from Takebe et al. (2018).

Figure 3: Examples of primary and secondary metabolites. Primary metabolites are D-glucose and vitamin A which are a carbohydrate and a lipid respectively. Secondary metabolites are 2,3,4-trichloro-7iodoindole and callophycol $C$, which are an indole and a meroterpenoid, respectively isolated by Dr Helen Woolner, VUW.

Figure 4: A schematic of the differences between spectroscopy-guided and bioactivity-guided isolation.

Figure 5: Examples of pyrroloiminoquinone alkaloids.

Figure 6: A depiction of how different drugs change the morphology of cells in different ways.

Figure 7: Acquisition of quantifiable data for cell morphology profiling. Adapted from Futamura et al (2012), with image segmentation images taken from an educational resource from cellprofiler.org/outreach.

Figure 8: Chemical structure of the six exemplar drugs used.

Figure 9: Taxol treatment for 48 hours skews the cell cycle to favour G2.

Figure 10: Zampanolide treatment skewed the cell cycle to favour S-phase and G2.

Figure 11: Doxorubicin treatment skewed the cell cycle.

Figure 12: BEZ235 treatment skewed the cell cycle to increase the percentage of cells in G1 and reduce the percentage in S-phase.

Figure 13: A66 treatment skewed the cell cycle to increase the percentage of cells in G1.

Figure 14: Temozolomide treatment did not change the percentage of cells in cell cycle phases.

Figure 15: Abundance of pAkt is reduced after BEZ235 treatment.

Figure 16: Abundance of pAkt is reduced after A66 treatment.

Figure 17: Taxol treatment increased percentage of affected and dead A549 cells in a concentration dependent manner.

Figure 18: Zampanolide treatment increased percentage of affected and dead A549 cells in a concentration dependent manner.

Figure 19: Doxorubicin treatment increased percentage of affected and dead A549 cells in a concentration dependent manner.

Figure 20: BEZ235 treatment increased percentage of affected and dead A549 cells in a concentration dependent manner.

Figure 21: A66 treatment did not affect and or kill A549 cells.

Figure 22: Temozolomide treatment did not affect and or kill A549 cells. 
Figure 23: Taxol treatment affected forward scatter (FSC) and cell granularity (SSC).

Figure 24: Zampanolide treatment affected forward scatter (FSC) and cell granularity (SSC).

Figure 25: Doxorubicin treatment affected forward scatter (FSC) and cell granularity (SSC).

Figure 26: BEZ235 treatment affected forward scatter (FSC) and cell granularity (SSC).

Figure 27: A66 treatment affected forward scatter (FSC) and cell granularity (SSC).

Figure 28: Temozolomide treatment affected forward scatter (FSC) and cell granularity (SSC).

Figure 29: Zampanolide treatment made cells larger and rounder in brightfield.

Figure 30: Doxorubicin treatment made cells larger in brightfield.

Figure 31: BEZ235 treatment made cells clump together and lose protrusions in brightfield.

Figure 32: Stained A549 cells imaged on the IN Cell Analyser 6500.

Figure 33: Stained A549 cells imaged on the Olympus FV3000.

Figure 34: Examples of image abberations made as a result of the autofocus function on the IN Cell Analyser 6500.

Figure 35: A549 morphology of nucleus and cell after treatment with 250 nM of Taxol and 25 nM of zampanolide.

Figure 36: A549 morphology of nucleus and cell after treatment with 62 nM of doxorubicin.

Figure 37: A549 morphology of nucleus and cell after treatment with $62 \mathrm{nM}$ of BEZ235 and $1 \mu \mathrm{M}$ of A66.

Figure 38: A549 morphology of nucleus and cell after treatment with $10 \mu M$ of temozolomide.

Figure 39: User Interface of CellProfiler with close up of modules.

Figure 40: General method of MCP specific to Te Herenga Waka.

Figure 41: Data output from CellProfiler pipeline depicting image segmentation of nuclei, cytoplasm and actin.

Figure 42: Examples of boxplots generated from morphological data generated by the optimised CellProfiler pipeline with images of a range of doxorubicin treated A549 cells from the Olympus FV3000.

Figure 43: CellProfiler Analyst user interface.

Figure 44: Extraction and isolation of Latrunculia kaakaariki.

Figure 45: Schematic of COSY, HSQC and HMBC correlations superimposed onto discorhabdin E.

Figure 46: Chemical structures of Discorhabdin $E$ and $G$.

Figure 47: Discorhabdin E treatment affected and killed A549 cells.

Figure 48: Discorhabdin E treatment affected forward scatter (FSC) and cell granularity (SSC).

Figure 49: A549 morphology of nucleus and cell after treatment with $1 \mu M$ and $2 \mu M$ of Discorhabdin $E$.

Figure 50: A549 morphology of nucleus and cell after treatment with formic acid at concentrations equivalent to the residual amounts in the $1 \mu M$ and $2 \mu M$ Discorhabdin $E$ samples. 
Appendix 1: Gating strategy for cell cycle assay.

Appendix 2: Gating strategy for cytotoxicity assay.

Appendix 3: Morphology parameters compared to PI content to understand how cytotoxicity related to morphological change.

Appendix 4: Code made by Danyl MacLaughlan for RStudio to transform CellProfiler quantified morphology data spreadsheet into box plots.

Appendix 5: Code made by Jeffrey Sheridan for RStudio to change file names from IN Cell Analyser 6500 to format CellProfiler regular expression in Metadata module can recognise.

Appendix 6: CellProfiler module parameters for morphology quantification.

Appendix 7: CellProfiler module parameters for quality control pipeline.

Appendix 8: Positive ion mass spectrometry data - Fraction 70A.

Appendix 9: Positive ion mass spectrometry data - Fraction $70 B$.

Appendix 10: Positive ion mass spectrometry data - Fraction 70C.

Appendix 11: Positive ion mass spectrometry data - Fraction 70D.

Appendix 12: Positive ion mass spectrometry data - Fraction $70 D$.

Appendix 13: ${ }^{13}$ C NMR of discorhabdin E.

Appendix 14: COSY spectrum of discorhabdin E.

Appendix 15: HSQC spectrum of discorhabdin E.

Appendix 16: HMBC spectrum of discorhabdin E.

Appendix 17: ${ }^{1}$ H NMR spectrum of pure discorhabdin E. 


\section{List of Tables}

Table 1: The estimated time and cost of the steps of the drug discovery and development pipeline. Adapted from Mohs \& Greig (2017).

Table 2: Comparison of the microscopes available to Te Herenga Waka researchers.

Table 3: NMR data of Discorhabdin E (d $\left.\sigma^{-D M S O}\right)$.

Table 4: Experimental and literature ${ }^{1} \mathrm{H}(600 \mathrm{MHz})$ and ${ }^{13} \mathrm{C}$ NMR data of Discorhabdin $E$ and $G$ in $\mathrm{CD}_{3} \mathrm{OD}$, and compared to Fraction $70 \mathrm{~A} / \mathrm{B}$. 


\section{List of Abbreviations}

3T3 Mouse fibroblast cell line

3T3-J2 Mouse embryonic fibroblast cell line

A549 Lung adenocarcinoma cell line

ACS American Chemical Society

APS Ammonium persulfate

BSA Bovine serum albumin

$\mathrm{CD}_{3} \mathrm{OD} \quad$ Deuterated methanol

CFSE Carboxyfluorescein succinimidyl ester

$\mathrm{CH}_{3} \mathrm{NO}_{2} \quad$ Nitromethane

$\mathrm{CO}_{2} \quad$ Carbon dioxide

COSY Correlated Spectroscopy

CPA CellProfiler Analyst

d6-DMSO Deuterated dimethyl sulfoxide

DAPI 4',6-Diamidino-2-Phenylindole

$\mathrm{ddH}_{2} \mathrm{O} \quad$ Double distilled water

DMSO Dimethyl sulfoxide

DNA Deoxyribonucleic acid

ECL Enhanced chemiluminescent

EDTA Ethylenediaminetetraacetic acid

ER endoplasmic reticulum

EtOH Ethanol

FBS $\quad$ Foetal bovine serum

FDA Food and Drug Administration

FSC Forward scatter

G1 Gap 1

G2 Gap 2 
GPU Graphic Processing Unit

$\mathrm{H}_{2} \mathrm{O} \quad$ Water

HEKTE Renal epithelium cell line

HeLa Cervical cancer cell line

HepG2 Human liver cell line

HIF-1 Hypoxia-inducible factor 1

HMBC Heteronuclear multiple bond correlation

HPLC High performance liquid chromatography

HRESIMS High resolution electron spray ionisation mass spectrometry

HRP Horse radish protein

HSQC Heteronuclear single quantum coherence spectroscopy

HTB-9 Human bladder carcinoma cell line

HUVEC Human umbilical vein endothelial cell line

IC50 Half maximal inhibitory concentration

IgG Immunoglobin $\mathrm{G}$

$\mathrm{K}_{2} \mathrm{PO}_{4} \quad$ Dipotassium hydrogen phosphate

$\mathrm{KCl} \quad$ Potassium chloride

LC-MS Liquid chromatography - mass spectrometry

M Mitosis

MCF-7 Breast cancer cell line

MCP Morphological Cell Profiling

$\mathrm{MeOH} \quad$ Methanol

$\mathrm{Na}_{2} \mathrm{HPO}_{4} \quad$ Disodium hydrogen phosphate

$\mathrm{NaCl} \quad$ Sodium chloride

NMR Nuclear Magnetic Resonance

pAkt Phosphorylated Akt

PBS Phosphate-buffer saline

PBS-T Phosphate-buffered saline supplemented with Tween

PCR Polymerase Chain Reaction 
PFG Pulse field gradient

PI Propidium iodide

PI3K Phosphoinositide 3-kinase

PVDF Polyvinylidene fluoride

Q-TOF Quadruple time-of-flight mass spectrometry

RAM Random Access Memory

RNA Ribonucleic acid

RT Room temperature

S Synthesis

SAR Structure activity relationship

SDS Sodium dodecyl sulfate

SEM Standard error mean

SH-SY5Y Neuroblastoma cell line

SSC Side scatter

TEMED Tetramethylethylenediamine

Tris Tris(hydroxymethyl)aminomethane

U2OS Osteosarcoma cell line

V Volts

VUW Victoria University of Wellington 


\section{Chapter 1: General Introduction}




\subsection{Drug Discovery}

Drug discovery and development is exceedingly expensive in time, money and resources. As shown in figure 1, the reason for this can be realised. In order for a drug candidate to become commercially available, it must pass the four main stages of this pipeline: basic research, preclinical development, clinical trials, and regulatory approval. ${ }^{1}$ Within these stages there are a number of phases which prove the drug candidate is efficacious and safe (examples of these phases are stated in figure 1). The drug only proceeds to the next step within the drug discovery and development pipeline if these tests are passed satisfactorily. Each test requires copious amounts of costly laboratory resources, and researchers to carry them out, who must be paid for their time. Overall, this adds up. The time and cost of each phase is shown in table 1. It is estimated one drug takes approximately 10-15 years to get through this pipeline and costs on average upwards of US $\$ 1.5$ billion per drug.

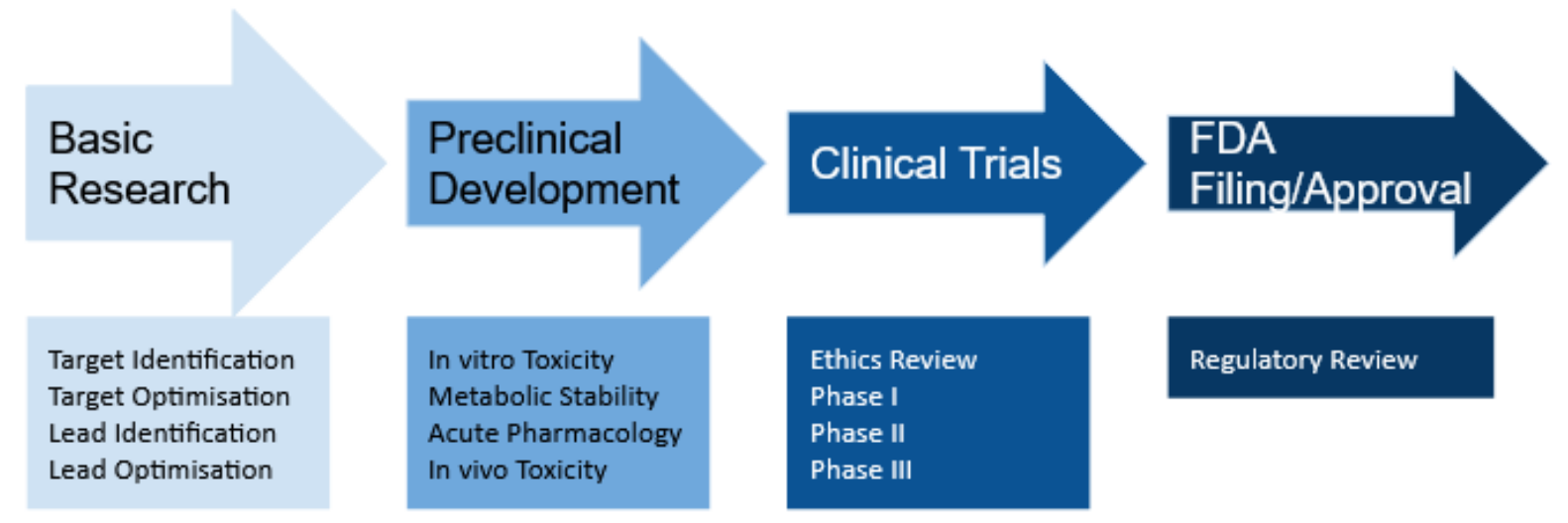

Figure 1. A schematic of the drug discovery and development pipeline. Examples of some of the procedures for each step are noted. Adapted from Mohs \& Greig (2017). 
Table 1. The estimated time and cost of the steps of the drug discovery and development pipeline. Adapted from Mohs \& Greig (2017).

\begin{tabular}{|c|c|c|c|c|c|c|c|c|c|}
\hline & $\begin{array}{c}\text { Target } \\
\text { to Hit }\end{array}$ & $\begin{array}{c}\text { Hit to } \\
\text { Lead }\end{array}$ & $\begin{array}{c}\text { Lead } \\
\text { Optimisation }\end{array}$ & Preclinical & Phase I & Phase II & Phase III & $\begin{array}{c}\text { Sub to } \\
\text { Launch }\end{array}$ & Total \\
\hline $\begin{array}{c}\text { Cycle } \\
\text { time (yrs) }\end{array}$ & 1.0 & 1.5 & 2.0 & 1.0 & 1.5 & 2.5 & 2.5 & 1.5 & 13.5 \\
\hline $\begin{array}{c}\text { Cost/ } \\
\text { Launch } \\
\text { (US } \\
\text { \$mil) }\end{array}$ & $\$ 94$ & $\$ 166$ & $\$ 414$ & $\$ 150$ & $\$ 273$ & $\$ 319$ & $\$ 314$ & $\$ 48$ & $\mathbf{\$ 1 , 7 7 8}$ \\
\hline
\end{tabular}

On top of this, the success rate of this pipeline is incredibly low. The percentage of drugs which made it through each phase from preclinical development to Food and Drug Administration (FDA) approval in the United States is shown in figure 2. Between 1991 and 2010 , only $6 \%$ of drugs which made it to the preclinical stage were approved by the FDA. ${ }^{2}$ Together with the data shown in table 1, this suggests that hundreds of billions of dollars are being spent on drugs not suitable for clinical use. ${ }^{1,2}$ For example, a drug that fails to enter Phase II trials will already have cost $\sim \$ 1$ b (table 1). ${ }^{1}$ Further, trends show that approvals for new drugs are stagnating, the cost of investment in research and development is increasing, and limitations such as regulatory burdens are tightening. ${ }^{3-6}$ Thus, innovation and technological advancements are a necessity to reduce the impact of these stressors on the pharmaceutical industry, and increase the discovery of breakthrough medicines. 


\section{Phase Success}

$100.00 \%$

\section{$87.50 \%$}

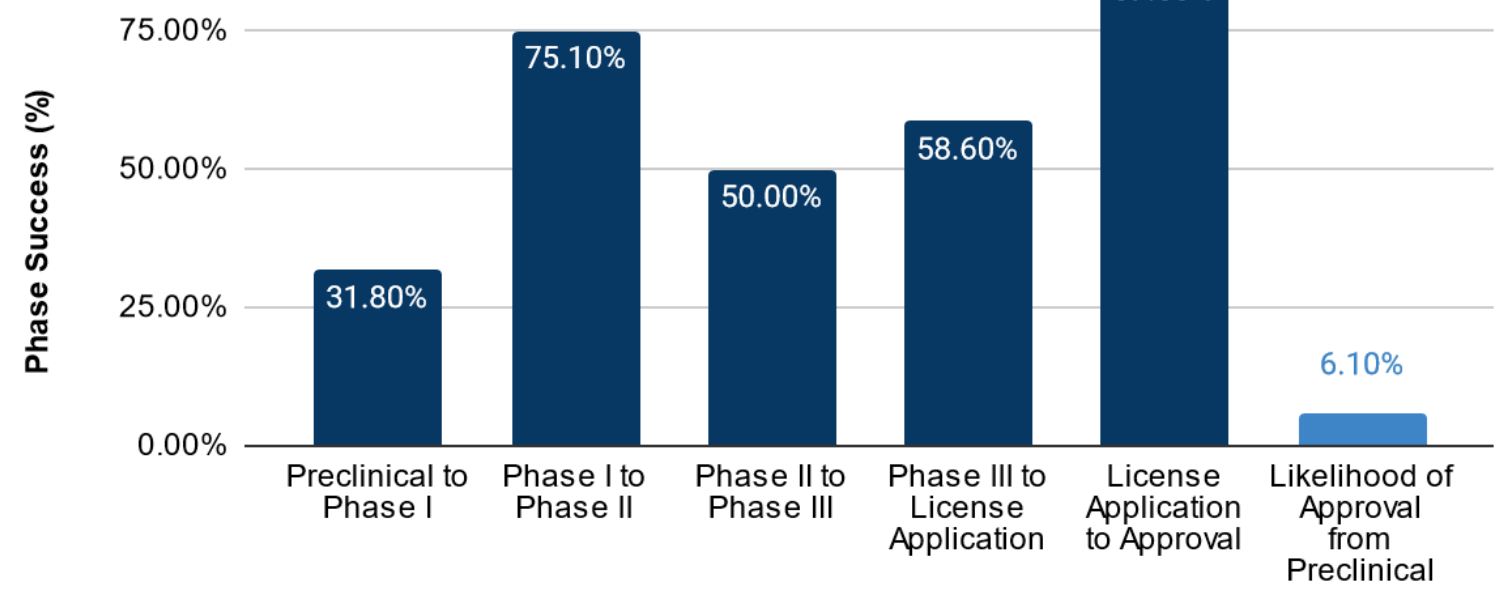

Phases

Figure 2. Phase Success of academic drug discovery and development between 1991 and 2010. Adapted from Takebe et al. (2018).

The purpose of the basic research stage within the drug discovery and development pipeline is to align a discovered compound with a primary medicinal purpose. The conventional approach to this is through four main steps: target identification, target validation, lead identification and lead optimisation. ${ }^{1,7}$ Target identification attempts to determine biological structures a drug candidate will bind to. ${ }^{7}$ Target validation confirms which biological structure it binds to. Then, exploration of the drug candidate's structures are synthesised and tested to identify Structure Activity Relationships (SAR). From this, multiple compounds with similar structures are synthesised. Lead identification selects which of these compounds binds most favourably to the target. This is then followed by lead optimization, where further, minor alterations are made to the lead drug candidate's structure to optimise favourable binding to the target.

During target identification, there are a large number of potential screens that could be carried out on drug candidates. Examples of these include SAR tests (i.e. affinity chromatography and reverse molecular docking), genomic methods (i.e. haploinsufficiency and homozygous profiling), and phenotypic methods (i.e. morphological cell profiling 
(MCP)). However, specific screening tests employed by each institution vary greatly. This is due to basic experimental limitations, for example, differences in compound discovery methods and the availability of the compound. There are also institutional restrictions such as resource availability, and the conservative use of established protocols. Consequently, the most efficacious screening techniques within the pharmaceutical industry are not regularly practised at educational/research institutes. Whilst this highlights deficits and issues within the drug discovery and development pipeline for the pharmaceutical industry, it also illustrates the constant need to try to implement innovative screening techniques within academic institutions.

Victoria University of Wellington - Te Herenga Waka (VUW) is no exception to these circumstances. In fact, due to the relative modesty of the institution's financial circumstances, the laboratories involved with drug discovery and development fall victim to the previously described limitations more frequently.

\subsection{Natural Products}

Researchers increase the discovery of successful drug candidates through enhancing compound discovery methods. Successful molecular pharmaceuticals are reputably sourced from either synthetic, natural or biotechnical backgrounds. Notably, approximately a quarter of all drugs that were approved from 1981 to 2019 are natural products or natural productderived. ${ }^{8,9}$ However, some chemists argue half of all drugs clinically used today are natural products, natural product-derived or natural product-inspired. ${ }^{10,11}$ This demonstrates nature is a vital source for drug candidacy.

Natural products are compounds produced by living organisms, such as plants, bacteria, fungi and marine organisms. For example, rapamycin was first characterised in 1975 from the bacteria Streptomyces hygroscopicus, and in 1999 it was FDA approved as an immunosuppressant. ${ }^{12,13}$ It is currently still being investigated for its anti-tumorigenic and anti-ageing properties. ${ }^{14-16}$

\subsubsection{Primary Vs Secondary Metabolites}

Natural products are labelled as primary or secondary metabolites based on their executive function within the living organism it is extracted from. Primary metabolites are compounds 
that are necessary for all organisms' survival such as carbohydrates and lipids whilst secondary metabolites are compounds which are not necessary for survival but enhance the producer's chance of survival or growth within their environment. Both metabolites have useful bioactive properties. However, secondary metabolites have a vast array of unique chemical scaffolds present as a result of the organism's evolutionary optimisation to its environment. Examples of primary and secondary metabolites are shown in figure 3. This figure shows the structural complexity of secondary metabolite's unique scaffolds easily juxtaposed to those of primary. Due to this, secondary metabolite extraction is believed by some researchers to supply more new molecular entities to the pharmaceutical industry than primary metabolites.

\section{Primary metabolites}<smiles>O=C[C@H](O)[C@H](O)[C@H](O)[C@H](O)CO</smiles>

D-glucose<smiles>CC1=C(/C=C/C(C)=C/C=C/C(C)=C/CO)C(C)(C)CCC1</smiles>

Vitamin A

Secondary metabolites<smiles>Clc1[nH]c2c(I)ccc(Cl)c2c1Cl</smiles>

2,3,4-trichloro-7-iodoindole<smiles>C=C1CC[C@@H]2[C@](C)(CCC(Cl)=C(C)C)[C@H](Br)CC[C@@]2(C)[C@@H]1Cc1cc(Br)cc(Br)c1O</smiles>

Callophycol C

Figure 3. Examples of primary and secondary metabolites. Primary metabolites are D-glucose and vitamin $A$ which are a carbohydrate and a lipid respectively. Secondary metabolites are 2,3,4-trichloro-7iodoindole and callophycol $\mathrm{C}$, which are an indole and a meroterpenoid, respectively isolated by Dr Helen Woolner, VUW. 
Secondary metabolites are found and isolated from the host organism through a screening method, often either bioactivity or spectroscopy/chemically-guided isolation. Bioactivityguided isolation is a method whereby extracts of an organism are fractionated and tested using a bioassay. Extracts that prove to be bioactive are then further assessed until the singular remaining isolate(s) is found and structurally determined spectroscopically. As shown in figure 4, using this approach a lot of bioactive compounds are found, however, very few are novel. Alternatively, spectroscopy-guided isolation is a method whereby compounds are isolated and characterised first using some chemical signature and then tested for bioactivity. Using this method, as shown in figure 4, very few bioactive compounds are found, however a large proportion of them are novel. So, despite drastically different approaches, it is believed both produce relatively equal and impressive yields of novel bioactive isolates.

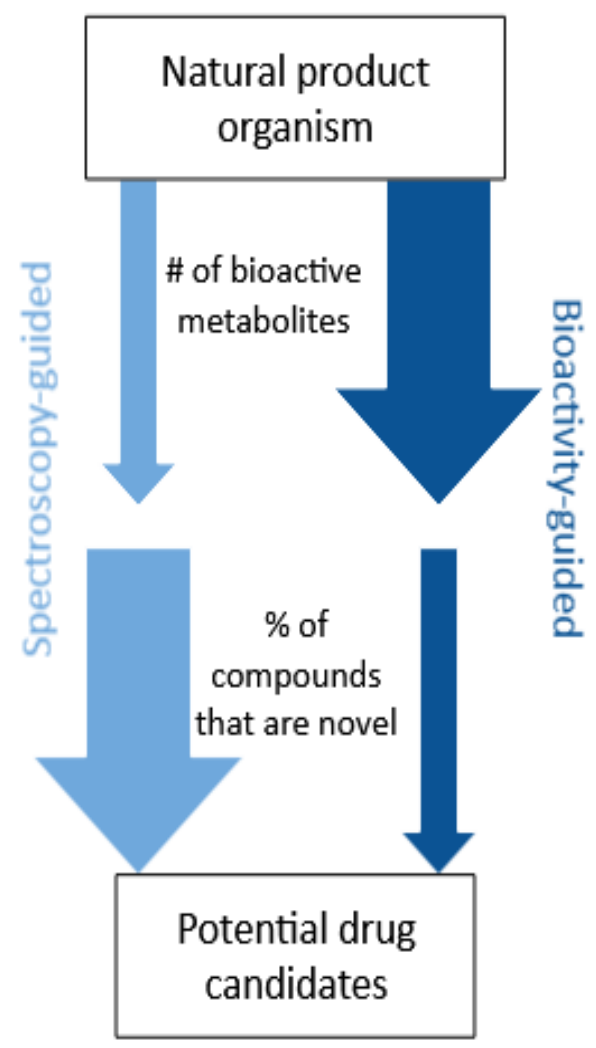

Figure 4. A schematic of the differences between spectroscopy-guided and bioactivity-guided isolation. 


\subsubsection{Natural products at Te Herenga Waka}

In the Natural Products laboratory at Te Herenga Waka, spectroscopy-guided isolation is the predominant isolation and characterisation method used to date. This laboratory has discovered numerous compounds over the past 15 years from Tongan and New Zealand marine algae, sponges and tunicates. For example, as part of her doctoral thesis submitted in 2017, Dr Helen Woolner isolated 15 new halogenated indoles (for example, 2,3,4-trichlor-7iodoindole in figure 3) from the red alga Rhodophyllis membranacea and six new meroterpenoids (for example, callophycol C in figure 3) from Tongan alga Callophycus serratus.

\subsubsection{Marine natural products}

The natural products laboratory has been successful in finding new compounds from marine organisms. ${ }^{17-25}$ The ocean makes up 70\% of the Earth's surface and contributes some of the most diverse and complex ecosystems in the world. ${ }^{26}$ With only $20 \%$ of the ocean floor mapped ${ }^{27}$ and already a rich diversity of unique organisms known within the ocean, ${ }^{28}$ it stands to reason that there are thousands more organisms or entire classes potentially even phylum yet to be discovered. With this, the potential for new bioactive compounds is immense. In the 2021 literature reviews of Marine Natural Products, the authors stated that 1490 new compounds were discovered in 2019 alone from marine organisms including bacteria, cyanobacteria, fungi, dinoflagellates, algae, sponges, cnidarians, molluscs, tunicates and echinoderms. ${ }^{29}$ For the purposes of this thesis I have focussed on marine sponges because, as mentioned above, they are a major research focus of the natural products laboratory at Te Herenga Waka.

\subsubsection{Marine Sponges}

Having lived on reef beds for 700 to 800 million years, marine sponges are the oldest extant multicellular animals in the world. ${ }^{30}$ They are metazoans that rely on their flagellates to inhale water in a unidirectional manner through epidermal filtering cells which "feed on" particulates such as bacteria. ${ }^{31}$ As of 2019 there are 15,000 species described, many of which are taxonomically characterised by the morphology of their skeletal spicules, which may also play a defensive role. ${ }^{30,32}$ Marine sponges are a particularly rich source of natural products with sponge-derived compounds comprising of $30 \%$ of all marine natural products discovered. ${ }^{30}$ Further, novel sponge-derived compounds are still being discovered today. 
From 2015 to 2020, 1403 new compounds sourced from marine sponges were reported. ${ }^{29}$ These compounds have extensively diverse structures, including alkaloids, terpenoids, peptides, polyketides, steroids and macrolides. Naturally, they have a diverse range of biological applications including antimicrobial, anticancer, antifungal, antiviral, antiinflammatory and antiparasitic activities. ${ }^{33-37}$ The reason these organisms contain such abundant and diverse bioactive secondary metabolites is not fully known. However, theories imply these are defence mechanisms to stop ingestion by carnivorous fish, fouling, and competition for space with other reef habitants. ${ }^{32}$ Other theories suggest that it is not necessarily the sponge itself that produces these compounds, but rather symbiotic bacteria using the sponge as a host producing secondary metabolites. ${ }^{38}$

\subsubsection{Latrunculia kaakaariki}

New Zealand's marine environment is thought to be home to a wide diversity of marine organisms, and hence natural products. Geographical isolation and evolutionary adaptation for millions of years has helped shape this unique marine environment. As a result, New Zealand is the perfect space to explore novel marine natural product compounds. In this thesis I have isolated compounds from the NZ marine sponge Latrunculia (du Bocage) kaakaariki. The genus Latrunculia is mostly found in the South Pacific, ${ }^{39}$ Antarctica $^{35,40}$ and the Red Sea. ${ }^{41}$ Interest in this sponge genus was sparked in 1975 when a fish toxin was discovered in the Red Sea sponge Latrunculia magnifica. ${ }^{42}$ More research in the 80 's found more cytotoxic components, most notably the macrolide latrunculin A. Latrunculin A is an actin fibre destabiliser with proven direct inhibition of G-actin polymerisation ${ }^{43}$ and is the most widely used actin modulator in molecular biology studies worldwide; it is commercially available from Sigma-Aldrich. Due to this, the drug had huge potential as an anti-cancer agent. This was because cytoskeletal actin polymerisation has a huge role in the direction of cellular protrusions, adhesion, and polarity, as well as the organisation of intracellular organelles. In cancer these functions can become abnormal and help metastasis, angiogenesis and cell division, thus the impediment of these cellular functions can restrain tumour growth. The latrunculin compounds are also known to suppress the activation of hypoxia-inducible factor 1 (HIF-1); another anti-cancer activity that impedes angiogenesis. ${ }^{44}$ They are also said to have antimicrobial effects. ${ }^{33}$ It should be noted that latrunculin A has not progressed into clinical use, nor does it appear to be in current clinical trials. 
Other compound families have been isolated from Latrunculia species. These include pyrroloiminoquinone alkaloids including discorhabdins; ${ }^{34}$ makaluvamines, the biosynthetic derivatives of discorhabdins; ${ }^{45}$ and tsitsikammamines, the non-halogenated pyrroloiminoquinones (figure 5). ${ }^{35}$ There are nine species of Latrunculia du Bocage currently known in NZ. ${ }^{46}$ In fact, discorhabdins A, B and C were initially discovered in New Zealand from Latrunculia sp. following a cytotoxicity bioassay-guided approach. ${ }^{39}$ Since then, NZ Latrunculia species have been extensively studied to find new and more bioactive derivatives of these compounds. $34,35,45,47,48$<smiles>O=C1C(Br)=CC2(C=CNC3C(=O)C4NC=C5CCN=C(C34)C52)C=C1Br</smiles>

Discorhabdin C<smiles>NC1=CC2=NCCc3c[nH]c(c32)C1=O</smiles>

Makaluvamine A<smiles>O=C1c2[nH]cc3c2C(=NCC3)c2c(-c3ccc(O)cc3)c[nH]c21</smiles>

Tsitsikammamine A

Figure 5. Examples of pyrroloiminoquinone alkaloids.

In this thesis, Latrunculia kaakaariki was investigated for novel pyrroloiminoquinone alkaloid secondary metabolites.

\subsubsection{Bioactivity tests}

A typical extension from the work done in the natural products laboratory at Te Herenga Waka is to run the novel compounds through a bioactivity assay. ${ }^{18,24}$ As the laboratory typically uses a spectroscopy-guided isolation, an isolated compound's biological activities are not known. The bioactivity tests conducted on the novel compounds may include, but are not limited to: cytotoxicity, proliferation and kinase inhibition screens, and antimicrobial and antifungal assays. However, the most commonly used is a cytotoxicity assay as it is the simplest and most cost effective. 


\subsection{Morphological Cell Profiling}

Having said that, Paracelsus' words "The dose makes the poison" exemplifies how cytotoxicity assays are not always a good indicator for bioactivity. Unfortunately, many compounds are screened for bioactivity using overly-simplistic cytotoxicity assays. If a drug increases cell death, the compound is said to be bioactive. However, a cell is a complex living thing which must utilise energy, transport molecules and reproduce. In order to do these tasks, there are many metabolic and signalling processes a cell must complete. In short, a cell can do much more than just die. When cells are exposed to drugs and compounds, they interact with these vital operations, thus a better screening approach would be one that tests for changes in a functional effect, and points towards a mechanism of action. There are a number of assays that effectively pinpoint a mechanism of action, however, most of them require complicated optimisation experiments which are only specific to that drug. One way this could be circumvented is to create an assay which is high-throughput, non-specific to the drug being tested, and has the capacity to not only conclude basic information about its bioactivity, but also distinguish a mechanism of action. One such method is a morphological cell profiling assay.

The technologies needed for an assay of this calibre are already often present in small chemical biology laboratories. All that is needed is a high-throughput confocal microscope, fluorescent stains for cellular components and cell culture capabilities. Te Herenga Waka has all the facilities above. Thus, the feasibility of establishing such an assay at the university is promising. Further, by doing this assay, the time, money and resources spent at the early stage of the drug discovery and development pipeline will be reduced. This is because the assay can aggregate the simple bioactivity tests necessary for the natural products laboratory's novel compounds and the target identification step from the drug discovery and development pipeline into one. Fundamentally, if implemented, its usefulness could be invaluable to the university. Due to this, this project attempted to assess the feasibility of establishing a morphological cell profiling assay at Te Herenga Waka.

\subsubsection{Morphological Cell Profiling assay development}

A morphological cell profiling assay is a quantitative and unbiased visual-based assay which utilises the theory that drugs with different mechanisms of action will cause different morphological responses in cells (figure 6). ${ }^{49-51}$ Thus, such an assay can identify a potential drug target from quantifying cell morphological features. ${ }^{45}$ There is a large array of 
morphological features that can be quantified using microscopy and imaging software, such as cell size and shape. ${ }^{50}$ Size is often measured as the area and perimeter of the cell. Shape is measured by multiple parameters, such as radius and diameter length, as well as circularity and ellipticity. In figure 7 the methodologies of acquiring this data can be seen. The basis of morphological cell profiling relies on the acquisition of a "Morphobase," that is, a database detailing drugs with known targets and the morphological changes they cause in the cells. ${ }^{52}$ Then, the morphological changes that occur with new compounds with unknown targets can be recorded and compared to the "Morphobase." If there are similarities between the output of the new compound with those of drugs with known targets, the new compounds target can be proposed as having a similar, if not the same target as the known drug. ${ }^{52,53}$

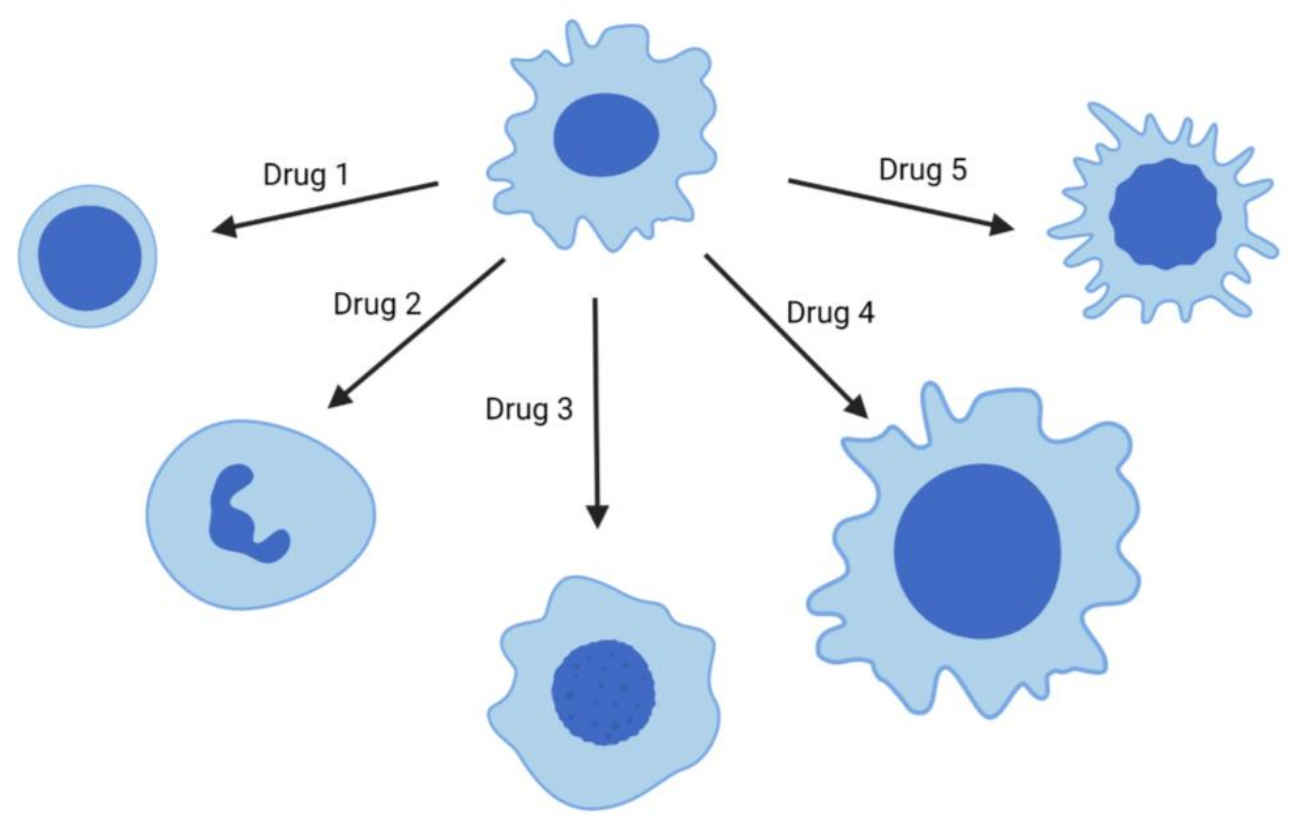

Figure 6. A depiction of how different drugs change the morphology of cells in different ways. 


\section{Image segmentation}
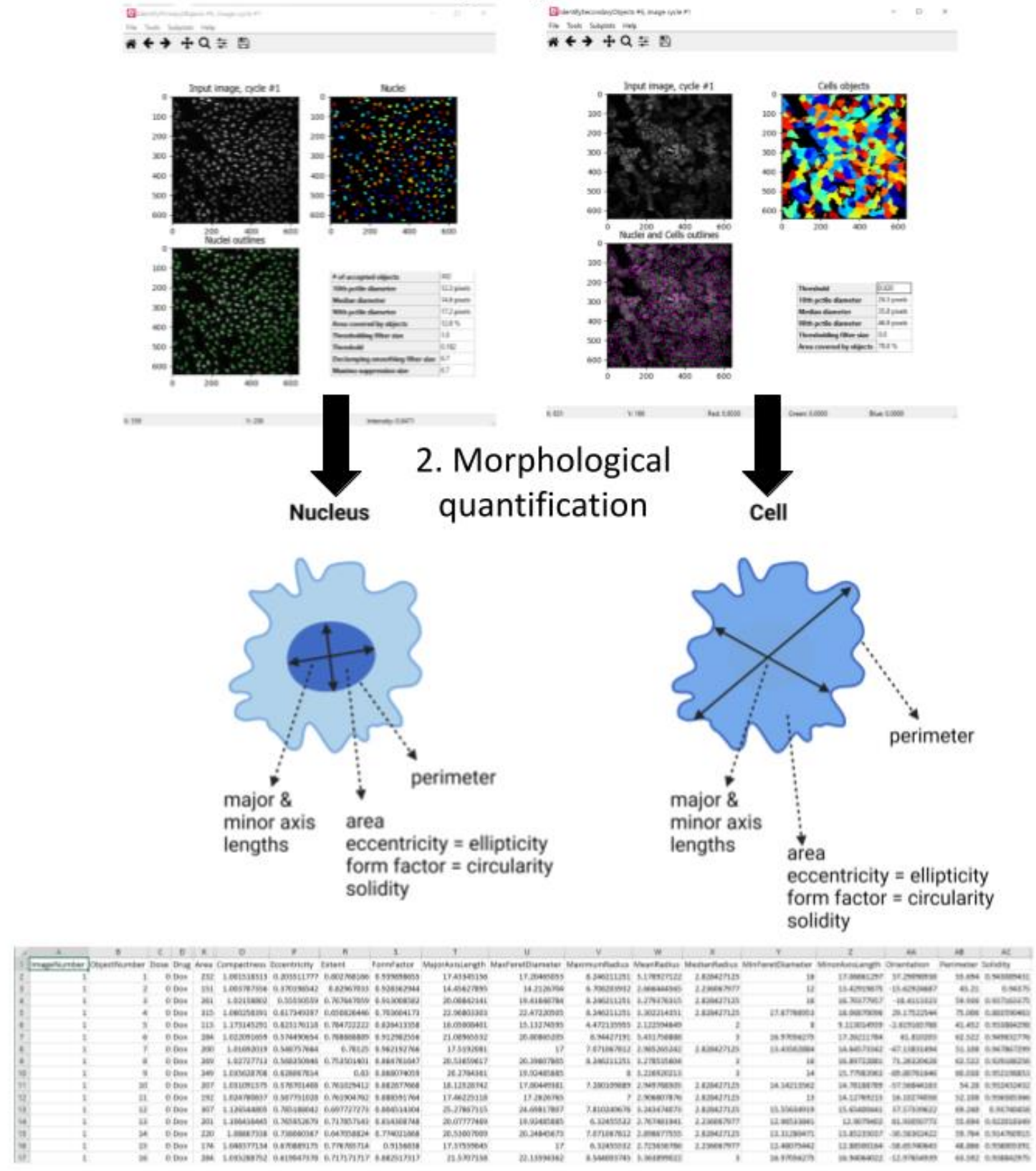

Figure 7. Acquisition of quantifiable data for cell morphology profiling. Adapted from Futamura et al (2012), with image segmentation images taken from an educational resource from cellprofiler.org/outreach.

Since the idea of a morphological cell profiling assay arose in the early 2000s, methodologies, software and instruments have advanced enough to make it possible. ${ }^{45-48}$ Microscopes have high-throughput and high-content capacity, modern software has extensive 
machine learning competence, and methods have been comprehensively optimised by experts in the field. Over the years, laboratories have attempted to create versions of the assay. ${ }^{52,54,55}$

For example, Sumiya et al. (2011) produced a small and target-specific version. ${ }^{54}$ To do this, they quantified two morphological variables; the area of the cytoplasm, and the distance between the centre of the nucleus and the centre of the cell. Additionally, they only analysed cellular responses for drugs which target structural proteins, that is, microfilaments and tubules. This bivariate analysis approach to morphological cell profiling, whilst simple, accurately identified the natural product compounds bisebromoamide and miurenamide $\mathrm{A}$ as actin filament stabilisers.

Other laboratories were more ambitious with their approach. By 2007, laboratories had the capability to conduct multivariate screening on single cells to analyse drug activity. Loo et al. (2007) analysed 276 morphological features on approximately 37 million cells to create their drug profile screen. ${ }^{55}$ These analyses, however, were very complex and computer dataprocessing intensive, thus making it infeasible to reproduce in all laboratories.

Futamura et al. (2012) created a simpler, high-content, multivariate cell morphology-based assay. ${ }^{45}$ This research defined what a morphobase is and evaluated fewer, but more biologically relevant cell morphological variables. A compound library of 207 drugs was used and the computer analysis appears more streamlined. This assay allowed researchers to look at bioactivity holistically. To do this, principal component analysis encompassed all morphological variables measured and grouped the drugs accordingly from the compound library with similar targets. It was then demonstrated that it correctly identified the targets of three compounds.

\subsubsection{CellProfiler}

Despite this early research, it was a cell phenotypic identification and quantification software called CellProfiler which revolutionised morphological cell profiling assays and propelled it to the forefront of drug discovery and development. ${ }^{56}$ The software uses a customisable module system to construct a pipeline personalised for any laboratory's phenotypic analysis needs. ${ }^{50,56-59}$ In a morphological cell profiling context, a pipeline can be customised to accurately identify and segment specific cellular components which have been stained with fluorescent dyes. From this, the cellular components can be morphologically quantified. 
In 2016, Bray et al. first highlighted the softwares applicability for a morphological cell profiling assay ${ }^{60}$ Whilst this paper only demonstrates that software could differentiate between drug treated and control cells based on differences in morphological features after analysis had been completed, it does suggest a cell morphology bioassay could be created with this method and technology. In 2019, the assay was established in a small chemical biology laboratory. ${ }^{61}$ This highlights the feasibility of the method for other small chemical biology laboratories, such as at Te Herenga Waka.

\subsubsection{CellProfiler Analyst}

An important element to this research is quality control of the images produced from the microscope. When images are acquired through a high-throughput method, the autofocus function is not entirely accurate. This results in image aberrations, saturation issues and blurriness. The consequence of these image defects will cause segmentation in CellProfiler to be incorrect and thus the morphological variables quantified will be inaccurate. To fix this, a sister software to CellProfiler, called CellProfiler Analyst (CPA), was created. This software has a machine learning algorithm within it called a classifier. It works by utilising an image quality measurement pipeline database created in CellProfiler, and then requiring the user to manually sort images into classes usually based on the user's opinion of saturation and blurriness in each image. Once enough images have been manually quality controlled, the software has the capability to flag all sub-standard images and extract them from future CellProfiler pipeline analysis. Thus, this efficiently enables accurate quantification of cellular morphological variables in all the remaining images.

\subsubsection{Cell Painting Assay}

A morphological cell profiling assay can quantify multiple cellular components morphology with CellProfiler, if careful selection of fluorescent dyes is made prior to imaging. This process has been optimised by the creators of CellProfiler for the purposes of morphological cell profiling assays. The dye list and protocol used is now described as a cell painting assay. The original goal of a cell painting assay was to gather as much morphological data about the cell without harming the cells integrity or disrupting the ability for image-based quantitative analysis to be performed. ${ }^{14-16}$ This assay was first described in 2013 by Gustafsdottir et al. ${ }^{62}$ It used six stains and five fluorescent channels to visualise seven cellular components with a confocal microscope. The cellular components were the nuclei, nucleoli, endoplasmic reticulum (ER), Golgi apparatus, actin filaments, mitochondria, and the plasma membrane. 
The assay was then optimised in 2016 by Bray et. al. and slight changes to the stains used were made. ${ }^{60}$ Instead, only five stains were used and cytoplasmic RNA would also be detected. Since then, the new protocol has been used a number of times to characterise small molecules and find their potential mechanism of action. ${ }^{1,12,15}$

Bray et. al. also showed that this generic staining protocol can be used on multiple cell lines. ${ }^{60}$ These include but are not limited to U2OS, A549, MCF-7, 3T3, HTB-9, HeLa, HepG2, HEKTE, SH-SY5Y, HUVEC, primary human fibroblasts and primary human hepatocytes/3T3-J2 fibroblast co-cultures. This highlights that the assay is cell type diverse. Bray et al. stated that the only change to the assay required between cell types is the seeding density due to the differences in proliferation rate and cell death that drugs will have on different cell types. They recommended the optimal seeding density is one where individual cells infrequently overlap by the time of image analysis. They also recommended an adherent cell line with a flat morphology be used.

\subsubsection{Issues with previous literature}

In previous literature there is no appropriate protocol stating how to determine the best concentration for each drug for the morphobase for morphological cell profiling. Some have standardised the concentration of drugs used, whilst others have adopted complex mathematical algorithms that are infeasible to reproduce. Some even, such as Bray et al., do not give any explanation as to how the best concentration was determined. In Svenningsen \& Poulsen (2019), ${ }^{61}$ the work from the small chemical biology laboratory, three concentrations of drugs were used to create a morphobase. They were $100 \mathrm{nM}, 1 \mu \mathrm{M}$ and $10 \mu \mathrm{M}$. They declared multiple concentrations were used to overcome nonspecific drug effects, such as toxicity, often seen at $10 \mu \mathrm{M}$ - the maximum concentration typically used to assess bioactivity of novel compounds in chemistry journals. However, this standardisation of drugs using multiple concentrations is still not successful in overcoming this issue, as drugs have huge varying effects and toxicities. It is unlikely that even one of these three concentrations tested will capture the cell morphology most representative of the drug's activity. A better approach would be to test each drug individually and find a "Goldilocks" concentration in which it is active, but not toxic.

One way this issue could be overcome is through flow cytometry as it can measure cytotoxicity and basic morphological characteristics, such as cell size and granularity can be measured from the same sample. ${ }^{7}$ It is also a high-throughput method which can detect these 
features in a few seconds per sample, requires minimal sample preparation, and the software for analysis is easy to use. Due to these qualities, it was used for determining a drugs Goldilocks concentration for an image-based morphology assay.

\subsection{Aims}

In this thesis, the following was addressed:

1. Explored the feasibility of a flow cytometry-based method to determine the Goldilocks bioactive concentration on drugs and novel compounds for morphological cell profiling

2. Examined the feasibility of establishing a morphological cell profiling assay at Te Herenga Waka

3. Isolated compounds from Latrunculia kaakaariki and used those compounds as proof of principle the methods established in the first two results chapters are practical. 
Chapter 2: Materials \& Methods 


\subsection{Biology}

\subsubsection{Cell Culture}

A549 cells (ATCC@ CCL-185 ${ }^{\mathrm{TM}}$ ) are an adherent epithelial carcinoma cell line originally obtained from the lung of a 58-year-old Caucasian male. They were cultured in DMEM (Gibco, Life Technologies) supplemented with 5\% foetal bovine serum (FBS) (Gibco, Life Technologies) and 1\% glutamax (Life Technologies).

Cells were grown in T75 cell culture flasks and incubated in a humidified incubator (Thermo Scientific) at $37^{\circ} \mathrm{C}$ with a $5 \% \mathrm{CO}_{2}$ conditioned atmosphere. Confluency was checked using a phase-contrast inverted microscope (Olympus IX51, Olympus, Japan). When 80\% confluency was reached, cells were passaged into a new T75 flask. Growth media was aspirated and cells were washed with $2 \mathrm{~mL}$ PBS (Life Technologies). Trypsin-EDTA (Life Technologies) ( $2 \mathrm{~mL}, 0.05 \%)$ was then applied to the cells and immediately aspirated. Cells were then incubated until they had lifted off the flask surface. Typically, A549 cells would require approximately 10 mins. Once lifted, $10 \mathrm{~mL}$ media was applied to the cells to neutralise the trypsin. A proportion of cells were transferred into a new T75 flask and supplemented with media to make the final volume $12 \mathrm{~mL}$.

All cells in culture were handled in sterile conditions in a Logic Labconco® Biological safety cabinet (Total Lab Systems Ltd.). The apparatus used within the cabinet was sprayed with $70 \%$ EtOH (Pure Science in distilled $\mathrm{H}_{2} \mathrm{O}$ ) before use.

Cells were regularly tested for mycoplasma by PCR testing (e-MYCO, Intron Biotech)..

\subsubsection{Storage}

All cells not actively cultured were stored in $1 \mathrm{~mL}$ cryovials in a liquid nitrogen cryostorage tank (Thermo Scientific).

\subsection{Freezing Down Cells}

To prepare the cells for long term storage, freezing media consisting of $70 \%$ media, $20 \%$ FBS, 10\% DMSO (Sigma Aldrich) was made. Cells were resuspended in the freezing media to a density of $1 \times 10^{\circ}$ cells $/ \mathrm{mL}$ and aliquoted at $1 \mathrm{~mL} / \mathrm{vial}$ into cryovials. The cryovials were then added to the CoolCell ${ }^{\circledR}$ Cell Freezing Containers and brought down slowly to $-80^{\circ} \mathrm{C}$. After 4-24 hours, the vials were transferred into liquid nitrogen for long term storage. 


\subsection{Thawing Cells}

When a cell line was needed for culture the desired cell vial was retrieved from the liquid nitrogen cryostorage tank. It was rapidly thawed in a $37^{\circ} \mathrm{C}$ water bath, transferred to $9 \mathrm{~mL}$ of pre-warmed media in a $15 \mathrm{~mL}$ centrifuge tube and inverted slowly. Cells were centrifuged at 400xg for 4 mins on a centrifuge (Gyrozen). Once pelleted, the media was aspirated to remove as much DMSO as possible. The cell pellet was then resuspended in fresh growth media and transferred to a T25 flask. A549 were left to incubate for at least 72 hours before media was replaced due to their fragility post-thawing.

\subsubsection{Treatments}

Drugs used were doxorubicin (obtained from Wellington Regional Hospital pharmacy, dissolved in saline for use in patients), temozolomide (Selleck Chemicals, dissolved in DMSO), Taxol and zampanolide (both gifted by Professor John Miller, VUW; dissolved in EtOH), BEZ235 and A66 (both gifted by Professor Peter Shepherd, University of Auckland; dissolved in DMSO).

\subsubsection{Microscopy Preparation}

\subsubsection{Histogrip}

Coverslips (22 x $22 \mathrm{~mm}$ ) (BioStrategy) were treated with (3-aminopropyl)triethoxysilane (Histogrip) (99\%, Sigma Aldrich) to ensure cells could adhere to the glass surface of the coverslip. Coverslips were placed into a beaker and covered in a Histogrip:acetone solution (1:50) and were then placed on a rotary shaker (115 rpm, $5 \mathrm{mins})$. Two acetone washes were completed following Histogrip treatment. The slides were then finally washed with EtOH and placed into a Schott bottle with $\mathrm{EtOH}(100 \%)$ until the coverslips were required.

Histogrip treated coverslips were placed into a 6-well plate with EtOH and incubated for at least one hour. The EtOH was aspirated and the coverslips were washed with sterile PBS. Cells were then plated onto the coverslip, which were subject to treatment (section 2.1.2) and staining (section 2.1.3.2). A small drop of DAPI containing mounting media was used to mount the coverslip, and clear nail polish used to seal and fasten the coverslip to the slide. 


\subsubsection{2. $\quad$ Staining}

\subsection{DAPI}

4',6-diamidino-2-phenylindole (DAPI) was used to stain DNA. Mounting media containing DAPI (VECTASHIELD) was used for all nuclei staining and stored in the fridge. DAPI was used as per the manufacturers instructions.

\subsection{CFSE}

CellTrace $^{\mathrm{TM}}$ Carboxyfluorescein succinimidyl ester (CFSE) Cell Proliferation Kit (Life Technologies) was used to stain cytoplasmic proteins before the cells were fixed and permeabilised. Anhydrous CFSE was removed from a $-20^{\circ} \mathrm{C}$ freezer and dissolved in DMSO at $5 \mathrm{mM}$. PBS was added to washed cells at $1 \times 10^{\circ}$ cells $/ \mathrm{mL}$. CFSE $(1 \mu \mathrm{L} / \mathrm{mL}, 5 \mu \mathrm{M})$ was then added and incubated for 20 mins before cells were washed and resuspended in growth media.

\subsection{ActinRed}

Molecular Probes ActinRed 555 ReadyProbes Reagent (Thermo Scientific) was used to stain actin filaments. Cells were fixed and permeabilised by EtOH (100\%) prior to use. ActinRed ${ }^{\mathrm{TM}}$ reagent was added, $1 \mathrm{drop} / \mathrm{mL}$, into growth media, and cells were incubated in ActinRed for 30 mins. Following incubation, the cells were washed with 1x PBS.

\subsection{CellMask}

Invitrogen $^{\mathrm{TM}}$ CellMask ${ }^{\mathrm{TM}}$ Deep Red Plasma membrane stain (Invitrogen ${ }^{\mathrm{TM}}$ C10046) was used to stain plasma membrane. A working solution of the stain was prepared 1000x in media. $1 \mathrm{~mL}$ of the working solution was added to each well on a 6 well plate prior to the cells being fixed and permeabilised. The cells were incubated for $10 \mathrm{mins}$ before being washed with PBS.

\subsubsection{General cell preparation}

Once 6-well plates had been prepped with Histogrip-treated coverslips, cells were plated at $5 \%$ confluency to ensure they would not be more than $50 \%$ confluent on the day of fixation. Cells were incubated for 24 hours and checked whether cell adhesion to the coverslip had occurred. They were then treated with the relevant drug and left to incubate for 48 hours. 
After drug treatment, the cells were stained, fixed and permeabilised in solvent. Whether the stain was applied before or after fixation and permeabilisation is described in each protocol.

Fixation and permeabilisation utilised cold $\mathrm{EtOH}(70 \%)$. To fix and permeabilise the cells media was aspirated and then washed with PBS. Cold EtOH $(70 \%, 2 \mathrm{~mL})$ was then added to each well and left at RT for 2 mins. The EtOH was immediately aspirated and the cells washed with PBS. Staining protocol could then immediately continue.

Once staining was completed the coverslip could be secured to a frosted microscope slide (25 x $75 \mathrm{~mm}$ x $1 \mathrm{~mm}$ ) (Thermo Scientific). A drop of DAPI mounting media was applied to the slide $1 \mathrm{~cm}$ from the top of the non-frosted slide of the slip. This was done to ensure that the coverslip was placed in identical places across slides for automation of the image acquisition process. The coverslip was then placed cell side down using tweezers by carefully dragging it along the surface of the slide until it reached the mounting media droplet. This should cause the mounting media to spread evenly along the coverslip edge. The coverslip could then be dropped gently onto the slide. If the coverslip slid along the slide surface, tweezers could be used to gently adjust its position. A KimWipe ${ }^{\mathrm{TM}}$ was then placed over the coverslip and slide and gently pressed down. This was done to remove any air bubbles from under the coverslip and to remove excess mounting media. Clear nail polish was then administered to every corner of the coverslip and left to dry. Once dry the clear nail polish could be thinly applied along the edges of the coverslip to secure it. Once dry the slides were then stored in slide boxes to ensure there was no light exposure.

\subsubsection{Hardware}

\subsubsection{Microscopes}

Three microscopes with fluorescent capabilities were used.

Olympus FV3000 is a laser-scanning confocal microscope. Protocol was optimised to the following: 9 images were taken per slide with a 40x magnification lens. 15 planes for a Zstack were taken per image between set focal planes which encompassed the whole cell. These focal planes were manually optimised for every slide taking into consideration all stain's channels used. Scan size (resolution) was 800 x 800 pixels. Non-sequential mode was used. 
The Olympus Slide Scanner VS200 is a high-throughput fluorescent microscope. Images were taken randomly over the slide with protocol optimised by Alfonso Schmidt, Malaghan Institute of Medical Research.

The IN Cell Analyser 6500 HS (GE Healthcare/Cytiva) is a high-content laser-scanning confocal microscope. Protocol was optimised to the following: 100 images per stain were taken at one focal plane per slide with a 10x magnification lens. The focal plane was optimised using the autofocus tool on the DAPI stained cells. The other stain's channels would automatically focus from this parameter being set, provided the focal plane offset was manually optimised for each channel. The resolution quality of the image was predetermined by the Confocal. Exposure time was also optimised for each stain and the images were automatically taken in a sequential manner.

\subsubsection{Computers}

Image analysis was carried out using a Windows 10 Workstation running 2 Intel Xeon 2.2 GHz processors with 64 GB RAM and 64 GB shared GPU memory.

Additional analysis using Cell Profiler was carried out on a Windows 2016 Server running 2 Intel Xeon $2.3 \mathrm{GHz}$ processors with $64 \mathrm{~GB}$ RAM.

\subsubsection{Software}

\subsubsection{Image Analysis Software}

Morphological analysis was conducted with three software. They were CellProfiler ${ }^{\mathrm{TM}}$ 4.1.3, CellProfiler ${ }^{\mathrm{TM}}$ Analyst 2.2.0, and Image (FIJ).

\subsubsection{RStudio}

Morphological data output was analysed by RStudio. R transformed the Excel spreadsheets generated by CellProfiler into boxplots using code written by Danyl McLaughlan (appendix 1).

RStudio was also required to transform file names automatically generated from the IN Cell Analyser 6500 confocal to a format CellProfiler could recognise to extract metadata from. This code was written by Jeffrey Sheridan (appendix 2). 


\subsubsection{Flow Cytometry}

All flow cytometry data was obtained on a FACSCanto II (BD, Bioscience, USA) and data was analysed using FlowJo v.10 software (TreeStar Inc. OR, USA).

\subsubsection{1. $\quad$ Cell Cycle Assay}

Cells were plated at 5\% confluency in 6-well plates and incubated for 24 hours to allow adhesion to the plate. They were then treated for 48 hours with the relevant concentrations of drug.

Following treatment, the cells were trypsin digested and transferred into a $15 \mathrm{~mL}$ centrifuge tube and centrifuged in a RT centrifuge for 5 mins at 400xg. The cells were washed with PBS and centrifuged. The subsequent pellet was resuspended in $300 \mu \mathrm{L}$ of PBS. Concurrently, 5 mL Eppendorf tubes with $700 \mu \mathrm{L}$ of ice-cold EtOH (100\%) were prepared. The $300 \mu \mathrm{L}$ cell/PBS solution was then added dropwise to the EtOH Eppendorf tube whilst vortex mixing to fix and permeabilize the cells in their current cell cycle state and allow for PI stain penetration. The cells were left for 30 mins, or up to 3 weeks in the $4^{\circ} \mathrm{C}$ fridge. Before staining cells were centrifuged (Eppendorf) at RT for 5 mins at 400xg and the EtOH/PBS solution was aspirated off.

To stain the cells a solution containing PI (1:500 of $5 \mathrm{mg} / \mathrm{mL}$, Sigma) and RNase A (1:40 of $10 \mathrm{mg} / \mathrm{mL}$ in $10 \mathrm{mM}$ TrisC $\mathrm{pH}$ 7.5/15 mM NaCl, Thermo Scientific) was made up in PBS and used to resuspend the cell pellet. The cells were transferred into FACS tubes (Corning), incubated at $37^{\circ} \mathrm{C}$ for $15-30$ mins before flow cytometry analysis. Cells were identified on the basis of forward scatter/side scatter, and DNA content determined on the PE-A channel. Single cells were gated using PE-A vs PE-W (appendix 3), and the cell cycle phases were determined using the in-built FlowJo v10 algorithm, based on DNA content.

\subsubsection{Cytotoxicity Assay}

Cells were plated at 5\% confluency in 6 well plates and treated with drugs at relevant concentrations for 48 hours prior to trypsin digestion. Once the cells were lifted they were pelleted, washed with PBS, pelleted again and then resuspended in a PI/PBS solution and transferred to a FACS tube. The cells were left on ice for $15 \mathrm{~min}$ to 2 hours before analysis to allow PI stain binding. Cells were identified on the basis of forward scatter/side scatter, and dead vs alive cells on the PE-A channel. Cells were gated using forward scatter to exclude 
debris or doublet cells. Dead cells were identified by the cluster of cells with high PE-A, and affected cells were categorised as any cell with a PE-A signal above the main PI-negative population (appendix 4).

\subsubsection{Western Blot}

Cells were serum starved for 24 hours to stop flux through signalling pathways. They were then treated with a drug at a relevant concentration for one hour or media for the control. FBS supplemented media was then applied for 15 mins to allow for signalling pathway activation. The cells were then washed with PBS twice and then lysed by pipetting the cells up and down at least 20 times in a lysis buffer $\left(50 \mu \mathrm{L} ; 70 \mathrm{mM} \mathrm{NaCl}, 20 \mathrm{mM}\right.$ Tris, $0.1 \%$ IGEPAL, Halt ${ }^{\mathrm{TM}}$ Protease Inhibitor Cocktail (100x)). The cells were left on ice for one hour to allow for lysis to occur. The lysed cells were then centrifuged (Gyrozen) at $4^{\circ} \mathrm{C}$ for 5 mins at $400 \mathrm{xg}$. The supernatant was then transferred into a fresh Eppendorf tube and then either used immediately or frozen at $-80^{\circ} \mathrm{C}$.

Protein levels were quantified to ensure the same amount was being analysed between the control and treatment samples using the Qubit ${ }^{\circ}$ Protein Assay Kit. After protein quantification, $50 \mu \mathrm{g}$ of sample was transferred into another Eppendorf tube. Laemmli Buffer (3x; 3\% SDS, $15 \%$ beta-mercaeptoethanol, $30 \%$ glycerol, $0.1 \mathrm{M}$ Tris $\mathrm{pH} 6.8$, and $0.01 \%$ of bromophenol blue) was added into each tube. The protein samples were then heated to $95^{\circ} \mathrm{C}$ for 5 mins.

Gels were created before protein quantification. Gel casting cassettes (Bio-RAD) were made up and loaded with resolving and stacking gels respectively until set. Resolving gel $(8 \mathrm{~mL}$ $\mathrm{ddH}_{2} \mathrm{O}, 5 \mathrm{~mL}$ 1.5 M Tris 8.8, $200 \mu \mathrm{L}$ 10\% SDS, $6.66 \mathrm{~mL}$ acrylamide, $100 \mu \mathrm{L} \mathrm{10 \%} \mathrm{APS,} 10$ $\mu \mathrm{L}$ TEMED) was made and transferred into glass plates in the casting cassette. Isopropanol was then placed gently into the glass plate to cover the gel. Once the resolving gel was set,

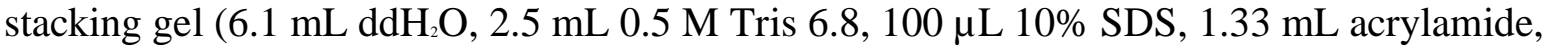
$50 \mu \mathrm{L} 10 \%$ APS, $10 \mu \mathrm{L}$ TEMED) was transferred into the glass plates on top of the resolving gel. A $1.5 \mathrm{~mm}$ comb was then immediately added to create wells. The gel was left for approximately 30 mins to set.

Running and Transfer buffers were also cooled in a $4^{\circ} \mathrm{C}$ refrigerator before protein quantification. Running buffer was made with $120 \mathrm{mM}$ glycine, $40 \mathrm{mM}$ Tris, $0.1 \%$ (w/v) 
SDS and $\mathrm{ddH}_{2} \mathrm{O}(1 \mathrm{~L})$. Transfer buffer was made with $120 \mathrm{mM}$ glycine, $40 \mathrm{mM}$ Tris, $20 \%$ (v/v) $\mathrm{MeOH}$ and $\mathrm{ddH}_{2} \mathrm{O}(1 \mathrm{~L})$.

Protein samples and a PageRulerTM Plus Prestain Protein Ladder ( $5 \mu \mathrm{L}$, Thermo Scientific) were added to wells. Gels were run for approximately one hour at $160 \mathrm{~V}$ in a gel tank (BioRAD) until the DNA ladder was well separated.

The protein was transferred onto a PVDF membrane (Immobilon ${ }^{\circledR}$ ). The membrane was cut to an appropriate size and activated using $100 \% \mathrm{MeOH}$. A tray was filled with transfer buffer and a sandwich plate with 2 sponges placed into the tray. The stacking gel was removed from the gel and placed on top of filter paper on the black side of the sandwich plate's sponge. The activated membrane was placed on top of the gel, followed with more filter paper. The sandwich plate was closed and placed into a balanced gel tank. Transfer was conducted at 20 $\mathrm{V}$ in the cold room overnight.

The membrane was then stained with Ponceau stain $(0.2 \%(\mathrm{w} / \mathrm{v})$ Ponceau S, 3\%(v/v) acetic acid) for 5 mins on a rocker. This was followed by a PBS-T (137 mM NaCl, $2.7 \mathrm{mM} \mathrm{KCl}, 10$ $\mathrm{mM} \mathrm{Na}_{2} \mathrm{HPO}_{4}, 1.8 \mathrm{mM} \mathrm{K}_{2} \mathrm{PO}_{4}, 0.01 \%(\mathrm{v} / \mathrm{v})$ Tween 20) until a signal was seen. Then the membrane was blocked for $1 \mathrm{hr}$ at room temperature on a rocker with a 5\% BSA/PBS-T solution.

A pAkt (S473) rabbit antibody (Cell Signalling Technology) was made up in the 5\% BSA/PBS-T at a 1:1000 dilution in a container. The membrane was rocked in this solution for an hour at room temperature. The membrane was then washed three times for 15 mins each with PBS-T ( 10 mL). A 1:7000 dilution of goat anti-rabbit horseradish protein (HRP) IgG secondary antibody (BioLegend) was made in PBS-T, added, and left to rock for $1 \mathrm{hr}$ at room temperature.

An ECL substrate (1:1 of each reagent, ECL Plus Western Blotting Substrate Kit, Thermo Scientific) was made up for imaging. Membranes were dunked in the ECL substrate and placed on a clean tray to be imaged on the Amersham Imager 600 .

The membrane was then stripped of antibody with $5 \mathrm{~mL}$ of the Restore ${ }^{\mathrm{TM}}$ Western Blot Stripping Buffer (Thermo Scientific) on a rocker for 30 mins and reblocked with 5\% BSA/PBS-T. Blotting was repeated with a 1:10000 dilution of monoclonal $\beta$-actin mouse antibody (Sigma Life Sciences), detected with goat anti-mouse HRP IgG (BioLegend). 


\subsection{Chemistry}

Latrunculia kaakaariki (638 g) was collected on Dec $2^{\text {nd }} 2003$ at Princess Island, which is part of the Three Kings Islands group. It was collected using SCUBA at a depth of around 15 m. It was stored at $-18^{\circ} \mathrm{C}$ in the School of Chemical and Physical Sciences, VUW, until extraction. A voucher specimen (MNP7338) is stored there.

\subsubsection{NMR}

A $600 \mathrm{MHz}$ Varian Direct Drive spectrometer equipped with a $5 \mathrm{~mm}$ PFG dual broadband probe or a JEOL JNM-ECZ600R with a nitrogen cooled $5 \mathrm{~mm}$ SuperCOOL cryogenic probe were used to record the NMR spectra of all compounds $\left(600 \mathrm{MHz}\right.$ for ${ }^{1} \mathrm{H}$ nuclei and 150 $\mathrm{MHz}$ for ${ }^{13} \mathrm{C}$ nuclei).

The residual solvent peak was used as an internal reference for ${ }^{1} \mathrm{H}\left(\delta_{\mathrm{H}} 3.31, \mathrm{CD}_{3} \mathrm{OD} ; 2.50, d \sigma^{-}\right.$ DMSO $)$ and ${ }^{13} \mathrm{C}\left(\delta_{\mathrm{C}} 49.00, \mathrm{CD}_{3} \mathrm{OD} ; 39.52, d 6\right.$-DMSO) chemical shifts. Samples were quantified by ${ }^{~} \mathrm{H}$ NMR spectroscopy using an internal $\mathrm{CH}_{3} \mathrm{NO}_{2}$ standard and the acquisition parameters described by West. ${ }^{63}$ Standard pulse sequences supplied by the manufacturer were used for ${ }^{1} \mathrm{H},{ }^{13} \mathrm{C}$, COSY, HSQC and HMBC spectroscopy experiments as described throughout this research.

\subsubsection{Mass Spectrometry}

High-resolution electron spray ionisation mass spectrometric (HRESIMS) data were obtained with an Agilent 6530 Accurate Mass Q-TOF equipped with an Agilent 1260 HPLC system for liquid delivery. The samples were analysed using positive ion polarity and a previously established protocol by Dr Joe Bracegirdle.

Samples were transferred to mass spectrometry sample vials and reconstituted to an approximate concentration of $1 \mathrm{mg} / \mathrm{mL}$ Injections $(0.1 \mu \mathrm{L})$ of the sample were analysed by Liquid Chromatography-Mass Spectrometry(LC-MS). Data was analysed on Mass Hunter.

\subsubsection{HPLC}

HPLC purifications were carried out using a Rainin Dynamax SD-200 solvent delivery system with $25 \mathrm{~mL}$ pump heads with a Varian Prostar 335 diode array detector. 
Analytical $(4.6 \mathrm{~mm} \times 250 \mathrm{~mm}, 1 \mathrm{~mL} / \mathrm{min})$ or semi-preparative $(10 \mathrm{~mm} \times 250 \mathrm{~mm}, 4$ $\mathrm{mL} / \mathrm{min}$ ) octadecyl-derivatised silica (C18, $5 \mu \mathrm{m}, 100 \AA$ ) HPLC columns (Phenomenex) were used.

Solvents used were of HPLC grade (Sigma Aldrich and AK Scientific) and $\mathrm{H}_{2} \mathrm{O}$ was distilled.

\subsubsection{Benchtop Column Chromatography}

Reversed-phase column chromatography was completed using Supelco Diaion HP20 chromatographic resin.

Solvents were analytical grade (Sigma Aldrich and AK Scientific) and $\mathrm{H}_{2} \mathrm{O}$ was distilled.

\subsubsection{Isolation of pyrroloiminoquinones from Latrunculia kaakaariki}

\subsubsection{Extraction}

Latrunculia kaakaariki (100g) was cut into $\sim 2 \mathrm{~cm}^{3}$ pieces and extracted twice in $400 \mathrm{~mL}$ of $\mathrm{MeOH}$. The first extraction was left for 24 hours in a $4^{\circ} \mathrm{C}$ refrigerator, then filtered. The second extraction was left for a week in a $4^{\circ} \mathrm{C}$ refrigerator.

\subsubsection{2. $\quad$ Isolation (figure 44)}

A reversed-phase HP20 column $(160 \mathrm{~mL})$ was prepared by washing acetone $(500 \mathrm{~mL})$ and then $\mathrm{H}_{2} \mathrm{O}(500 \mathrm{~mL})$. The sponge methanolic extract was then cyclic loaded onto the column: the second extract was put through the column first, followed by the first extract. The eluents were combined and diluted by half with $800 \mathrm{~mL} \mathrm{H}_{2} \mathrm{O}$. The diluted eluent was then passed back through the column. The eluent was diluted by half again $\left(1600 \mathrm{~mL} \mathrm{H}_{2} \mathrm{O}\right)$ and passed through the column a final time.

The column was washed with $\mathrm{H}_{2} \mathrm{O}(480 \mathrm{~mL}$, discarded). Fraction one was eluted off the column with $480 \mathrm{~mL}$ of $30 \%$ acetone in $\mathrm{H}_{2} \mathrm{O}$ solution. Fraction two was eluted with $480 \mathrm{~mL}$ of $75 \%$ acetone in $\mathrm{H}_{2} \mathrm{O}$ solution. The column was subsequently dried for 20 mins with air. Fraction three was then eluted off the column into a round bottom flask with $480 \mathrm{~mL}$ of $100 \%$ acetone. The acetone was evaporated in vacuo to give $320.2 \mathrm{mg}$.

Fraction 1 and 2 were backloaded onto separate reversed-phase HP20 columns ( $80 \mathrm{~mL}$ each). Before backloading, the columns were washed with $200 \mathrm{~mL}$ of acetone, then $200 \mathrm{~mL}$ of $\mathrm{H}_{2} \mathrm{O}$. To backload, the eluents were put through their respective columns. Each was then diluted 
1:1 with $\mathrm{H}_{2} \mathrm{O}$, then passed through the columns again. This was repeated. The columns were dried for 20 mins with air and the fractions were collected by passing acetone $(240 \mathrm{~mL})$ through the column. The eluents were dried in vacuo. Fraction one had a mass of $55.9 \mathrm{mg}$, and fraction two had a mass of $162.1 \mathrm{mg}$. Fraction one and two were then analysed by mass spectrometry, of which fraction one appeared to contain pyrroloiminoquinone alkaloids.

\subsubsection{Fractionation of Discorhabdins}

Fraction 1 was re-dissolved in $\mathrm{MeOH}(1 \mathrm{~mL})$ and was passed through a syringe microfilter (Jet Biolfil $^{\circledR}, 13 \mathrm{~mm}$, nylon membrane, $0.22 \mu \mathrm{m}$ ) to remove precipitate. The remaining sample was purified on a semi-preparative C18 HPLC column $\left(\mathrm{MeOH} / \mathrm{H}_{2} \mathrm{O}\right)$ to yield 3 fractions. The combined samples $70 \mathrm{~A}$ and $\mathrm{B}(2.8 \mathrm{mg})$ were further purified on an analytical C18 HPLC column $\left(\mathrm{MeOH} / \mathrm{H}_{2} \mathrm{O}\right)$, yielding discorhabdin E $(0.44 \mathrm{mg})$. Combined samples 70 $\mathrm{C}$ and D (3.7 mg) were analysed by mass spectrometry to yield a potentially unknown discorhabdin species, however the compound was unstable and further analysis and purification could not be attempted.

\subsubsection{Discorhabdin E}

Discorhabdin E: purple solid; HRESIMS m/z $384.037[\mathrm{M}+\mathrm{H}]^{+}$(appendix 8 ); ${ }^{1} \mathrm{H}$ and ${ }^{13} \mathrm{C}$ NMR data, (table 3, figure 45 and appendices $12 \& 13$ ):

${ }^{1}$ H NMR (600 MHz, d6-DMSO) $\delta_{\mathrm{H}} 7.70(\mathrm{~d}, \mathrm{~J}=2.7 \mathrm{~Hz}, 1 \mathrm{H}, \mathrm{H}-1), 7.22(\mathrm{dd}, \mathrm{J}=10.0,2.7 \mathrm{~Hz}$, 1H, H-5), 7.05 (s, 1H, H-14), 6.22 (d, J = 9.7 Hz, 1H, H-4), 3.86 (dd, J = 14.9, 8.8 Hz, 1H, H17), 3.42 (m, 2H, H-8), 2.54 (td, J = 9.8, 3.2 Hz, 2H, H-16), 1.82 (m, 2H, H-7).

${ }^{13}$ C NMR (150 MHz, d6-DMSO) $\delta \mathrm{C} 178.6$ (C-3), 158.1 (C-19). 157.6 (C-1), 157.5 (C-5), 154. 9 (C-10), 124.9 (C-4), 124.8 (C-16), 122.9 (C-21), 122.2 (C-12), 122.0 (C-2), 117. 8 (C15), 101.4 (C-20), 49.9 (C-17), 44.5 (C-6), 37.5 (C-8), 34.0 (C-7), 18.4 (C-16). 


\section{Chapter 3: Development of a flow cytometry-based method to determine compound concentration for a MCP assay}




\subsection{Introduction}

In this chapter, the feasibility of using a flow cytometry-based method to determine the optimal bioactive concentration for compounds in a morphological cell profiling assay is described.

Researchers have previously standardised concentrations of compounds at which they immediately began imaging cells and analysing the morphological changes. Svenningsen \& Poulsen (2019) used three concentrations of $100 \mathrm{nM}, 1 \mu \mathrm{M}$ and $10 \mu \mathrm{M} .{ }^{61}$ Whilst this standardisation of compound concentrations is simple, it means the quantified and analysed morphological parameters of the cells may not be relevant to the bioactivity of the respective compound, or its mechanism of action. As every drug has a different level of bioactivity, a concentration that is cytotoxic for one drug, may not be cytotoxic for another. If these concentrations are used in a morphological cell profiling assay, for some drugs the morphology quantified is representative of cell death, while others show no bioactivity at all, despite the standard concentrations being chosen carefully. Thus, the concentration used is an important consideration to determine whether a compound is bioactive.

Further, the accuracy of using a standard concentration method to propose a compound's target has never been explored, thus it is not known how precise this data is at informing researchers of bioactivity or potential molecular/cellular targets. So, in the context of screening natural product compounds through a MCP assay, with the future intentions to develop this assay to have the capacity to propose a target for novel natural product compounds, it is vital that each compound used - whether setting up a 'morphobase' or screening novel compounds, is used close to the drug's most bioactive, but not cytotoxic, concentration as possible.

To address this, a new method to determine the concentration each compound should be used at to treat and image cells in a MCP assay was developed. Cells were exposed to six exemplar drugs with known effects. Flow cytometry was used to look at both cytotoxicity via dye exclusion, and at the basic morphological parameters of size and cytoplasmic complexity. Each drug was tested at a range of concentrations, and the data was used to attempt to identify a concentration that affected the cell without inducing death, that is, a "Goldilocks" concentration. 
All experiments used the cell line A549. This cell line was chosen due to its relevance in microscopy-based assays, due to its flat and uniform morphology.

Exemplar drugs were chosen, identified by their target, and split into classes accordingly. The drugs used were: microtubule-stabilisers, zampanolide and Taxol, ${ }^{64-73}$ DNA-damaging agents, doxorubicin and temozolomide ${ }^{74-78}$ and PI3K inhibitors, BEZ235 and A66 (figure 8). ${ }^{79,80}$ Temozolomide was used as a negative control - it requires a very high concentration for use in vitro, ${ }^{78}$ and has very little effect on morphology or viability of cells at lower concentrations.

\subsection{Aims}

1. Confirm the exemplar drugs are active

2. Create a flow cytometry assay to determine the optimal concentration for a compound to be used at for a MCP assay

3. Assess whether the flow cytometry morphology parameters add supplementary information to help determine the Goldilocks concentration of a novel natural product compound for an MCP assay. 
<smiles>COc1cccc2c1C(=O)c1c(O)c(C(C)(C)O[C@H]3C[C@@H](N)[C@H](O)[C@H](C)O3)c(C[C@H](O)C(=O)CO)c(O)c1C2=O</smiles>

Doxorubicin<smiles>Cn1c(=O)n(-c2ccc(C(C)(C)C#N)cc2)c2c3cc(-c4cnc5ccccc5c4)ccc3ncc21</smiles>

BEZ235<smiles>Cn1nnc2c(C(N)=O)ncn2c1=O</smiles>

Temozolomide<smiles>Cc1nc(NC(=O)N2CCC[C@H]2C(N)=O)sc1-c1csc(C(C)(C)C)n1</smiles>

A66<smiles>[CH][C@H](C/C(C)=C/[C@H]1CC(=C)O1)OC(=O)/C=C/C=C(/C)CC(=O)CCC[C@H]1CC(=C)C[C@H]([C@@H](O)NC(=O)/C=C\C=C\C)O1</smiles>

Zampanolide

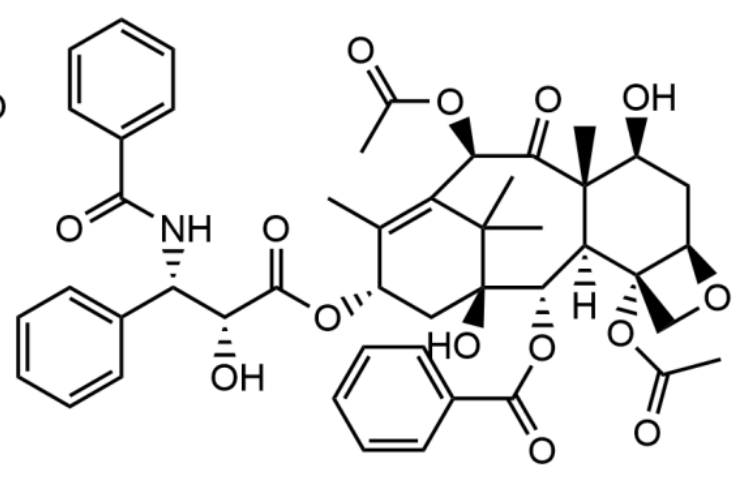

Taxol

Figure 8. Chemical structure of the six exemplar drugs used. 


\subsection{Results}

\subsubsection{Positive control for activity of compounds}

\subsubsection{1. $\quad$ Cell cycle analysis}

In order to check the drugs were active prior to morphological analysis, each was tested to determine the effect on cell cycle. Initially, this assay was used to assess the microtubule stabilisers, which are known to arrest cells in G2/M of the cycle. The assay was extended to all the drugs, as cell cycle arrest is a common response to cellular perturbation.

Cellular DNA content changes as the cell progresses through the different phases of the cell cycle. For example, cells in G2 will have twice the amount of DNA as cells in G1.

Conversely, cells in S phase have an intermediary amount of DNA between G1 and G2, as S phase is when a cell is actively synthesising DNA. A population of actively dividing cells has a distribution that makes a characteristic histogram, with the majority of cells in G1 at any given time (appendix 1).

The DNA content of A549 cells was measured with a fluorescent dye (propidium iodide, PI) that binds to nucleic acid. Cells were fixed and permeabilised in $70 \%$ ethanol, cellular RNA was broken down by RNAse digestion, followed by incubation in PI, which bound to the DNA. DNA content was determined by direct measurement of the fluorescent intensity of the PI stain in a minimum of 10,000 cells. The distribution of cells was determined and depicted as the proportion of cells in each cell cycle phase as a percentage of total cells measured.

DNA content was compared between treated and untreated cells. Concentrations for each drug were chosen based on IC $_{50}$ values from literature, with the exception of temozolomide. ${ }^{64,67,68,76,78,81-87}$ No tests were conducted with drug concentrations above $10 \mu \mathrm{M}$. This was based on the guidelines for bioactivity tests published in the American Chemical Society (ACS) Journal of Natural Products, ${ }^{88}$ which states that $10 \mu \mathrm{M}$ is the maximum concentration at which a compound should be deemed cytotoxic. Consequently, Temozolomide was not treated at concentrations close to the $\mathrm{IC}_{50}$ value as this is approximately $500 \mathrm{mM}$ in vitro for most cell types.

As expected, most of the untreated cells $(\sim 65 \%)$ were in $\mathrm{G} 1$ of the cell cycle. The remaining cells were split between S ( 10\%) and G2 ( 25\%) phases (figures 9 - 14) ${ }^{89}$ 
To confirm that the microtubule stabiliser Taxol was active, cells were treated with $250 \mathrm{nM}$ and $500 \mathrm{nM}$ for 48 hours (figure 9). Both $250 \mathrm{nM}$ and $500 \mathrm{nM}$ treatments showed an increase in proportion of cells in $\mathrm{G} 2$ from $\sim 25 \%$ to $\sim 83 \%$. The increased G2 was consistent with the action of a microtubule stabiliser. ${ }^{64}$

\section{Taxol Cell Cycle}

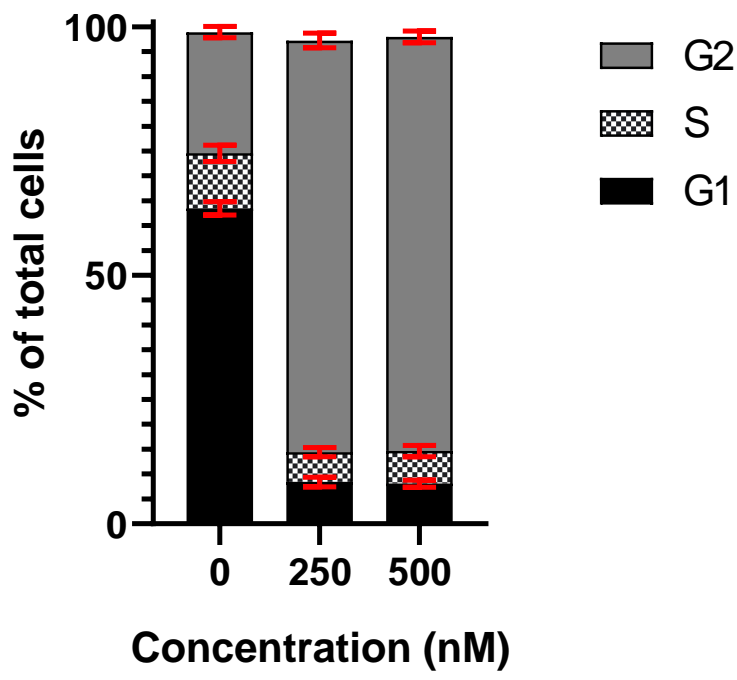

Figure 9. Taxol treatment for 48 hours skews the cell cycle to favour G2.

Concentration of drug treatment was plotted on the $x$-axis. The percentage of total cells in the analysed sample is plotted on the y-axis. 20,000 cells DNA content was analysed by flow cytometry PE-A channel per drug treatment. Proportions of cells in each cell cycle phase was determined with FlowJo's inbuilt cell cycle algorithm. The cell cycles are depicted by different shades: $G 1=$ black, $S=$ checker, $G 2=$ grey. The assay was run in technical duplicate and biological triplicate. Error bars $=$ Standard Error Mean (SEM) were determined on Excel, where $n=3$ (biological replicates).

Similar results were seen with the other microtubule stabiliser, zampanolide, which was tested at concentrations of $12 \mathrm{nM}$ and $25 \mathrm{nM}$. The cell cycle results (figure 10) confirmed bioactivity. As the concentration of treatment increased, the percentage of cells in G2 and S phase increased whilst G1 decreased. At $25 \mathrm{nM}, \sim 75 \%$ of cells were in G2 and S phase, compared to $\sim 35 \%$ in the untreated samples. A 1:1 ratio between G2 and S phase was consistent at all concentrations. These results were consistent with what you would expect from a microtubule stabiliser. However, the consistent 1:1 ratio between $\mathrm{G} 2$ and $\mathrm{S}$ phase with zampanolide treatment increases is an interesting observation as it is unlike what is seen with Taxol treatment. This could be due to different relative bioactive concentrations being tested. 
However it is more likely that despite both being microtubule stabilisers which bind to the taxane site of beta-tubulin, it is how they bind and to which specific protein residues which causes this difference. ${ }^{64,69,90}$ Zampanolide covalently binds, whilst Taxol does not. ${ }^{73,90}$ Another possibility the drugs have other secondary mechanisms of action that affect the cell cycle.

\section{Zampanolide Cell Cycle}

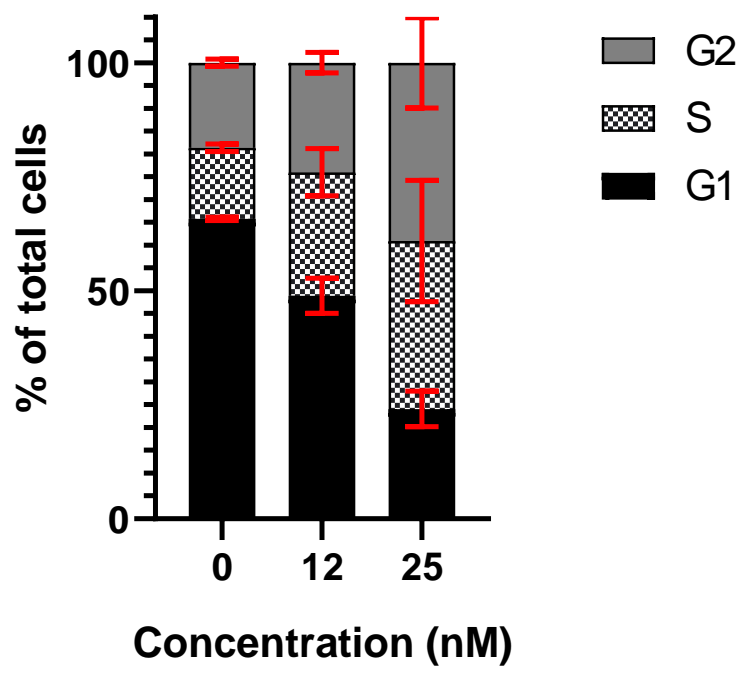

Figure 10. Zampanolide treatment skewed the cell cycle to favour S-phase and G2.

Concentration of drug treatment was plotted on the $x$-axis. The percentage of total cells in the analysed sample is plotted on the y-axis. 20,000 cells DNA content was analysed by flow cytometry PE-A channel per drug treatment. Proportions of cells in each cell cycle phase was determined with FlowJo's inbuilt cell cycle algorithm. The cell cycles are depicted by different shades: $G 1=$ black, $S=$ checker, $G 2=$ grey. The assay was run in technical duplicate and biological triplicate. Error bars $=$ Standard Error Mean (SEM) were determined on Excel, where $n=3$ (biological replicates).

Doxorubicin was used at $500 \mathrm{nM}$ and $1 \mu \mathrm{M}$. The doxorubicin cell cycle results (figure 11) showed both concentrations reduced the percentage of cells in S-phase to almost zero. Compared to untreated, which showed $\sim 10 \%$ of cells are in S-phase, doxorubicin caused a noticeable difference. The $500 \mathrm{nM}$ doxorubicin treatment showed the percentage of cells in G1 and G2 were evenly split between the two. This showed doxorubicin treatment increased the percentage of cells in G2, compared to the control, where the ratio is 2:1 These results are consistent with what you would expect from a DNA damaging agent. Doxorubicin inhibits topoisomerase II and in doing so induces many double strand breaks during 
replication. ${ }^{74,75,91,92}$ Due to these effects, the cell cycle arrests at the G2/M checkpoint that is pivotal to stop DNA-damaged cells replicating. ${ }^{91,92}$ At $500 \mathrm{nM}$ doxorubicin treatment, it is a reasonable assumption the increase in percentage of cells in $\mathrm{G} 2$ is accounted for by the G2/M checkpoint block. Thus, doxorubicin is most likely still viable as a topoisomerase II inhibitor. The $1 \mu \mathrm{M}$ doxorubicin treatment however, showed the proportion of cells in G1 and G2 is similar to the control, 2:1. This shift could be accounted for a number of reasons. For example, human error. However, it is more likely cell death occurred. A cell will be arrested in $\mathrm{G} 2$ at the G2/M checkpoint if only small amounts of DNA-damage occurs, but if DNA damage is too great, the cell will undergo cell death. It is rational to assume at $1 \mu \mathrm{M}$, too much DNA damage occurred and the drugs cytotoxic effects are seen instead. Considering cells with more DNA damage are more likely to arrest in G2 and subsequently die, the cell cycle phase ratios at $1 \mu \mathrm{M}$ may be similar to the control.

\section{Doxorubicin Cell Cycle}

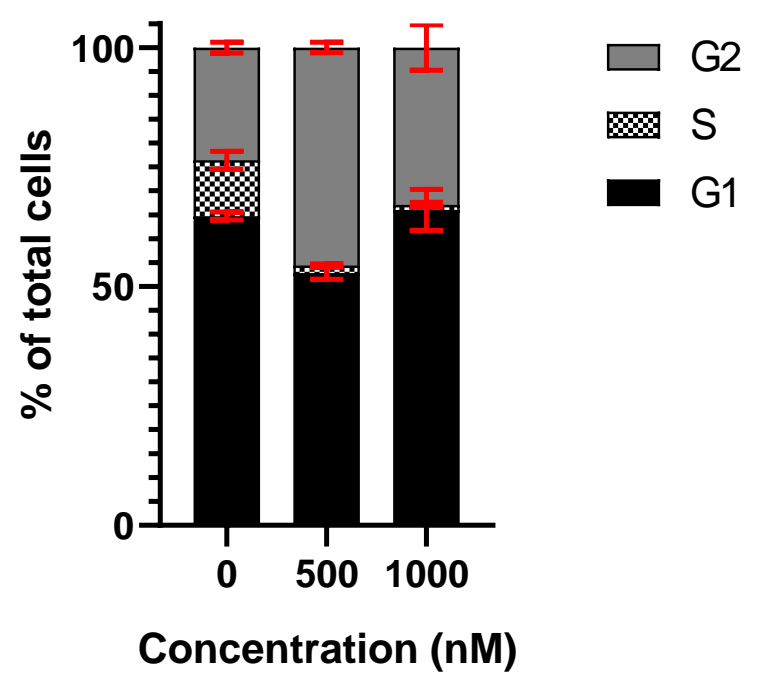

Figure 11. Doxorubicin treatment skewed the cell cycle.

Concentration of drug treatment was plotted on the $x$-axis. The percentage of total cells in the analysed sample is plotted on the y-axis. 20,000 cells DNA content was analysed by flow cytometry PE-A channel per drug treatment. Proportions of cells in each cell cycle phase was determined with FlowJo's inbuilt cell cycle algorithm. The cell cycles are depicted by different shades: $G 1=$ black, $S=$ checker, $G 2=$ grey. The assay was run in technical duplicate and biological triplicate. Error bars = Standard Error Mean (SEM) were determined on Excel, where $n=3$ (biological replicates).

BEZ235, a PI3K inhibitor was applied at $500 \mathrm{nM}$ and $1 \mu \mathrm{M}$, which led to over $90 \%$ of cells in G1 after BEZ235 treatment at both $500 \mathrm{nM}$ and $1 \mu \mathrm{M}$ (figure 12). This is a huge shift 
compared to the control, where only $\sim 65 \%$ of the cells were in G1. The reason for this shift can be explained by the drug's mechanism of action. The drug binds to the protein PI3K and inhibits the Akt cell signalling pathway. ${ }^{93}$ This pathway is involved in cell growth and proliferation. Specifically, it plays a role in G1/S transition and G2/M transition. ${ }^{94}$ Thus, if there was inhibition of this pathway, the cells may not be able to transition to the next phase. It is feasible BEZ235 inhibits cells from completing the G1/S transition at a slower rate than other cell cycle transitions, therefore stagnating the cells in G1. This confirmed BEZ235 is still active after years in the freezer, and shows activity consistent with a PI3K inhibitor, however, this needs to be confirmed further.

\section{BEZ235 Cell Cycle}

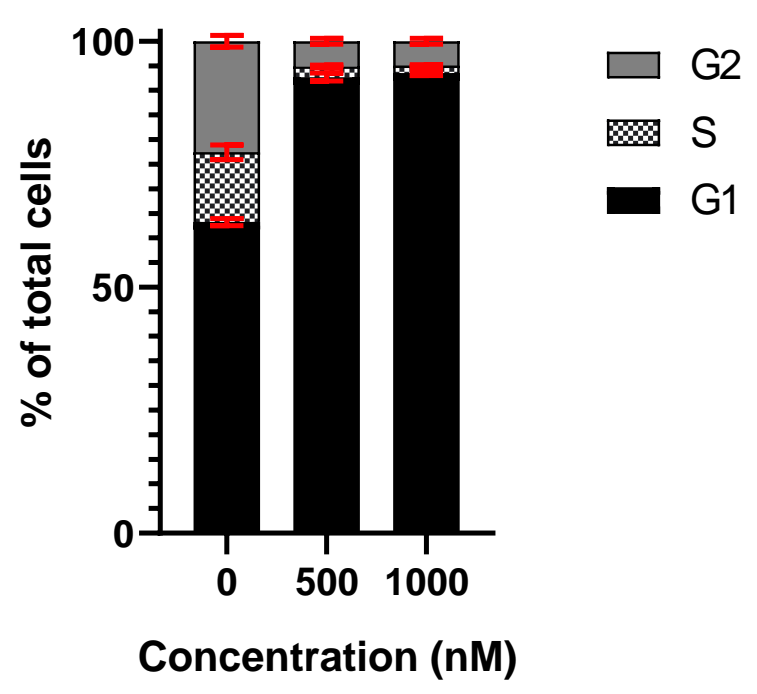

Figure 12. BEZ235 treatment skewed the cell cycle to increase the percentage of cells in G1 and reduce the percentage in $S$-phase.

Concentration of drug treatment was plotted on the $x$-axis. The percentage of total cells in the analysed sample is plotted on the y-axis. 20,000 cells DNA content was analysed by flow cytometry PE-A channel per drug treatment. Proportions of cells in each cell cycle phase was determined with FlowJo's inbuilt cell cycle algorithm. The cell cycles are depicted by different shades: G1 = black, $S=$ checker, G2 = grey. The assay was run in technical duplicate and biological triplicate. Error bars = Standard Error Mean (SEM) were determined on Excel, where $n=3$ (biological replicates).

A66, also a PI3K inhibitor, was tested at $500 \mathrm{nM}$ and $1 \mu \mathrm{M}$. The results from the cell cycle assay showed cell cycle shifts as the concentration increased (figure 13). Whilst these shifts were more subtle than BEZ235 at $500 \mathrm{nM}$ and $1 \mu \mathrm{M}$, the same trend towards G1 arrest can be 
seen. The percentage of cells in each cell cycle phase favoured an increase in G1 as concentration of A66 increased. A slight reduction in the percentage of cells in S-phase was seen as the concentration of A66 increased, also consistent with BEZ235 results. Together it confirms A66 is active.

\section{A66 Cell Cycle}

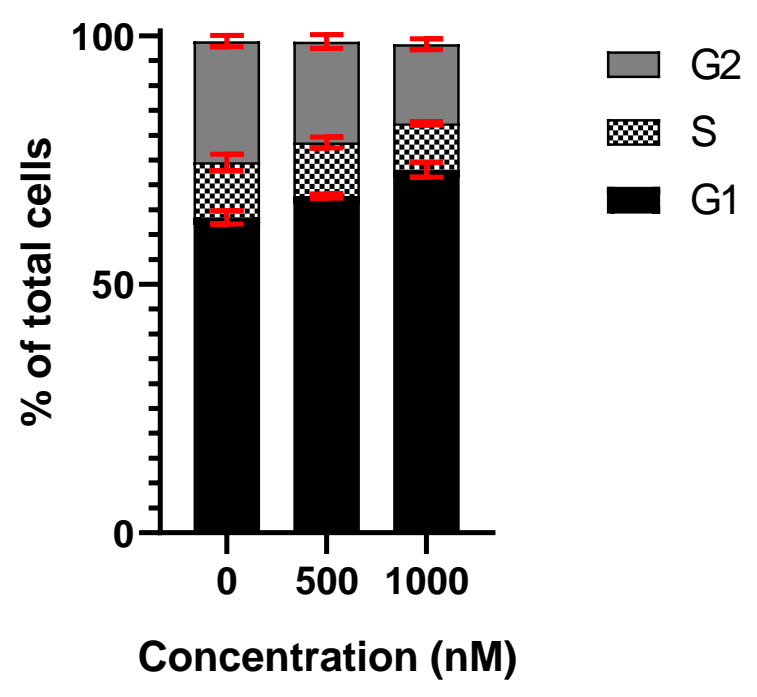

Figure 13. A66 treatment skewed the cell cycle to increase the percentage of cells in G1.

Concentration of drug treatment was plotted on the $x$-axis. The percentage of total cells in the analysed sample is plotted on the y-axis. 20,000 cells DNA content was analysed by flow cytometry PE-A channel per drug treatment. Proportions of cells in each cell cycle phase was determined with FlowJo's inbuilt cell cycle algorithm. The cell cycles are depicted by different shades: $G 1=$ black, $S=$ checker, $G 2=$ grey. The assay was run in technical duplicate and biological triplicate. Error bars = Standard Error Mean (SEM) were determined on Excel, where $n=3$ (biological replicates).

As previously mentioned, the $\mathrm{IC}_{50}$ values of temozolomide cited in previous literature were not used. Instead, $500 \mathrm{nM}$ and $1 \mu \mathrm{M}$ were used for the cell cycle assay. As expected, no difference was seen in cell cycle phases between the control and the temozolomide treated cells for the cell cycle assay (figure 14). 


\section{Temozolomide Cell Cycle}

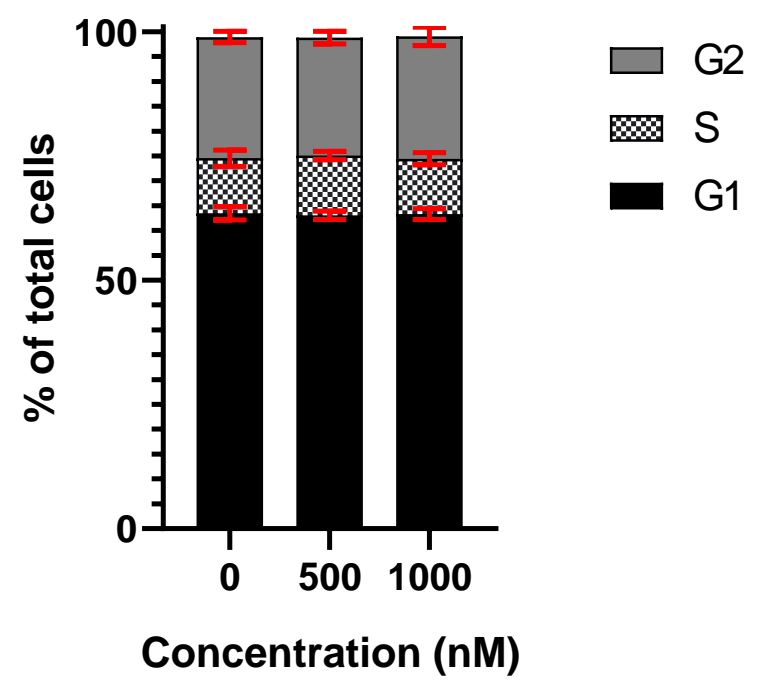

Figure 14. Temozolomide treatment did not change the percentage of cells in cell cycle phases.

Concentration of drug treatment was plotted on the $x$-axis. The percentage of total cells in the analysed sample is plotted on the y-axis. 20,000 cells DNA content was analysed by flow cytometry PE-A channel per drug treatment. Proportions of cells in each cell cycle phase was determined with FlowJo's inbuilt cell cycle algorithm. The cell cycles are depicted by different shades: $G 1=$ black, $S=$ checker, $G 2=$ grey. The assay was run in technical duplicate and biological triplicate. Error bars $=$ Standard Error Mean (SEM) were determined on Excel, where $n=3$ (biological replicates). 


\subsubsection{2. $\quad$ Inhibition of PI3K signalling using Western Blots}

The cell cycle assay results for BEZ235 and A66 confirmed the drugs had some activity but only BEZ235 was confirmed as an active PI3K inhibitor. To ensure the PI3K cell signalling pathway was inhibited, A549 cells were serum-starved overnight to stop flux through cell signalling pathways. Then BEZ added for one hour. The signalling pathways were reactivated with FBS supplemented media for 15 minutes before the cells were lysed. The phosphorylation of the Akt protein was analysed with and without BEZ treatment.

Phosphorylation of Akt occurs when PI3K is active. ${ }^{82,94}$ Therefore, if PI3K is inhibited there should be a reduction in the amount of pAkt within a cell.

In figure 15, there was a less intense Akt band in the BEZ235 treated lane, compared to untreated. The $\beta$-actin control was the same size for both samples. This indicates the drug is directly inhibiting the Akt pathway.

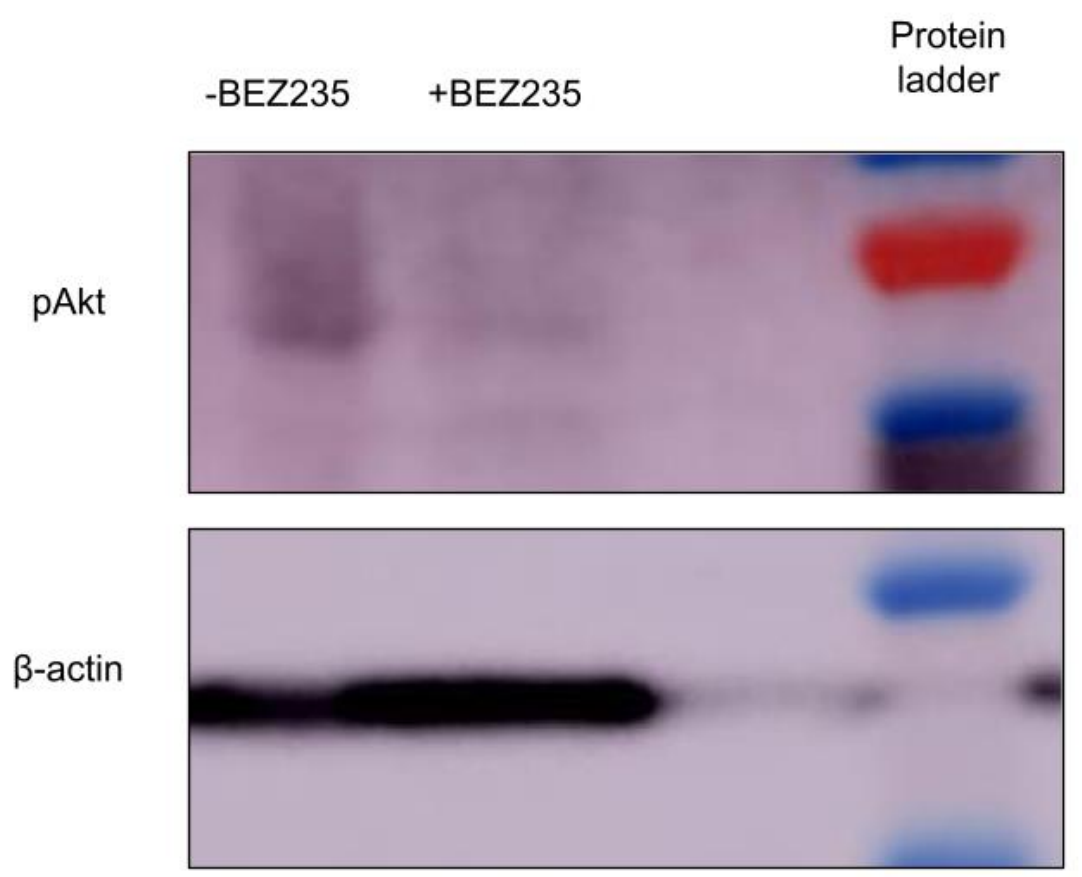

Figure 15. Abundance of pAkt is reduced after BEZ235 treatment.

Western blot for pAkt $(M W=60 \mathrm{kDa})$ was detected with an antibody specific to ser473P. $\beta$-actin $(M W=42$ kDa) was used as a control. Lane $1=$ control without BEZ235 treatment, lane $2=1 \mu$ M of BEZ235 treatment, Lane 3 = empty, Lane $4=$ protein ladder. In the pAkt blot the red band in lane 4 is $70 \mathrm{kDa}$ and the blue band below it is $55 \mathrm{kDa}$. In the $\beta$-actin blot the top blue band is $55 \mathrm{kDa}$ and the bottom blue band is $35 \mathrm{kDa}$. 
A preliminary attempt for A66 is shown in figure 16. Technical issues and time restrictions meant this Western blot was not conclusive nor repeated.

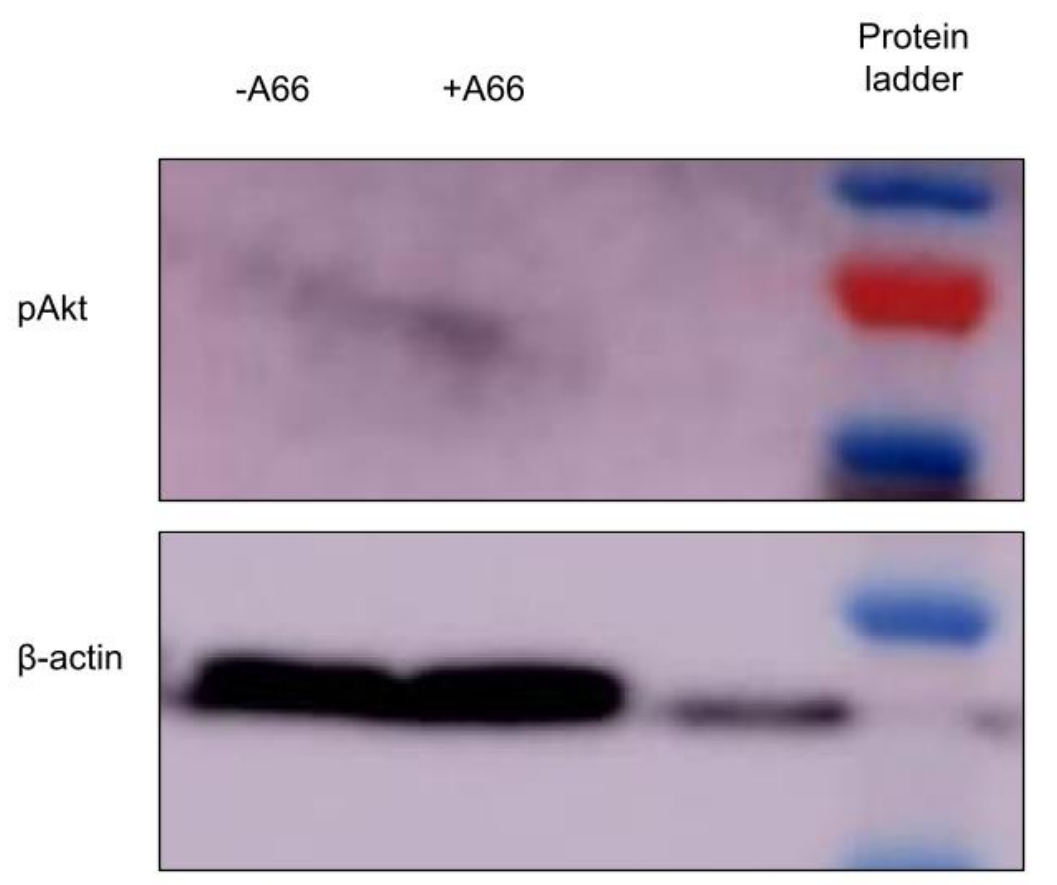

Figure 16. Abundance of pAkt is reduced after A66 treatment.

Western blot for pAkt $(M W=60 \mathrm{kDa})$ was detected with an antibody specific to ser473P. $\beta$-actin $(M W=42$ $k D a)$ was used as a control. Lane $1=$ control without BEZ235 treatment, lane $2=1 \mu \mathrm{M}$ of BEZ235 treatment, Lane $3=$ empty, Lane $4=$ protein ladder. In the pAkt blot the red band in lane 4 is $70 \mathrm{kDa}$ and the blue band below it is $55 \mathrm{kDa}$. In the $\beta$-actin blot the top blue band is $55 \mathrm{kDa}$ and the bottom blue band is $35 \mathrm{kDa}$.

\subsubsection{Cytotoxicity}

Cytotoxicity assays were carried out by flow cytometry to assess the drug's toxicity on A549 cells. This assay was undertaken to address concerns surrounding concentration standardisation seen in previous literature to determine a Goldilocks concentration. To do this, cytotoxicity of each drug was measured by examining cell viability. PI will permeate cells with compromised plasma membranes. This occurs when a cell is dead or stressed within its environment, for example, it is in media supplemented with a toxic drug. Flow cytometry measures PI fluorescent intensity within cells. High fluorescent intensity is analogous to non-viable cells, that is, dead as irreversible plasma membrane permeabilization has occurred. Conversely, viable cells have a no PI uptake and a low fluorescent intensity as 
their plasma membranes are unimpaired. Cells which are stressed will have an intermediary fluorescent intensity between the two, a phenomenon presumably due to low levels of plasma membrane permeabilisation. The gating strategy is shown in appendix 2. First cells were gated to remove debris. 'Dead cells' were identified and gated as a cluster of cells with the highest stain uptake. 'Affected cells' included both 'dead cells' and stressed cells which had taken up an intermediary amount of stain compared to the control. Concentrations for each drug were chosen based on $\mathrm{IC}_{50}$ values from literature, again with the exception of temozolomide.

Taxol was tested at a range of concentrations between 62 to $1 \mu \mathrm{M}$. The cytotoxicity assay results for Taxol showed it is cytotoxic (figure 17). As the concentration increased, the percentage of dead cells in the sample increased. The percentage of affected cells also increased in a concentration dependent manner. These results suggested what concentration the drug began to be active.

Taxol Affected Cells

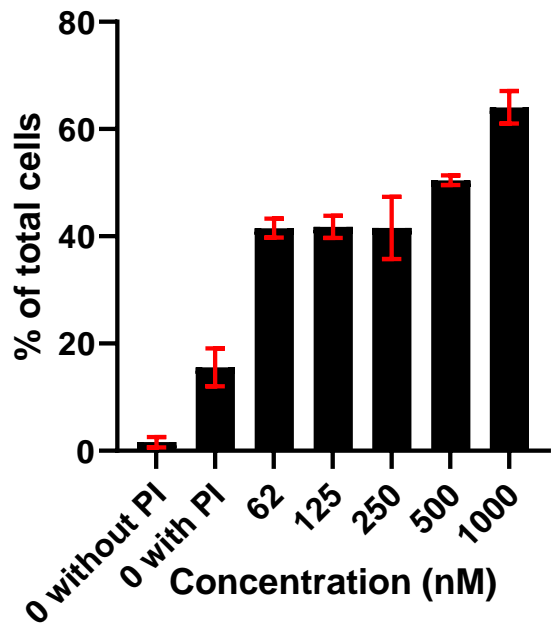

Taxol Dead Cells

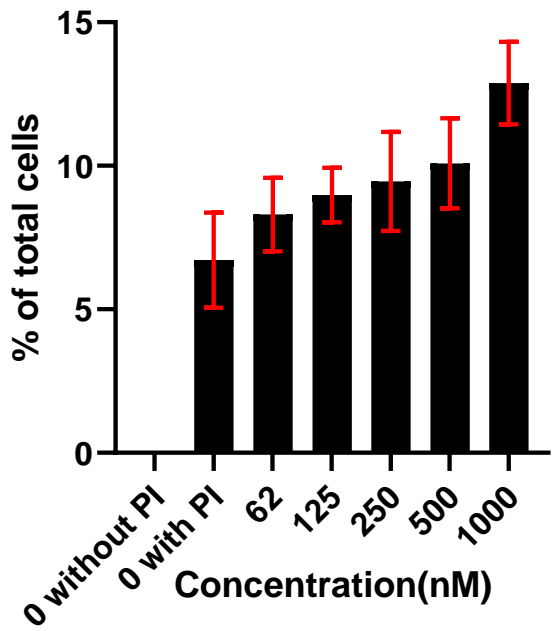

Figure 17. Taxol treatment increased percentage of affected and dead A549 cells in a concentration dependent manner.

Concentration of drug treatment was plotted on the $x$-axis. The percentage of total cells in the analysed sample is plotted on the $y$-axis. 20,000 cells per drug treatment were analysed by flow cytometry with the PEA channel. Results were acquired by flow cytometry. Error bars = SEM; Controls with and without PI were made to assist with gating. 
Zampanolide was tested at a range of concentrations between 6 to $50 \mathrm{nM}$. As the concentration of zampanolide increased, the percentage of dead and affected cells increased (figure 18). This showed that zampanolide is notably cytotoxic at concentrations greater than $50 \mathrm{nM}$ as the SEM bars of the $50 \mathrm{nM}$ treatment no longer overlapped with the SEM bars of the control with PI treatment.

Zampanolide Affected Cells

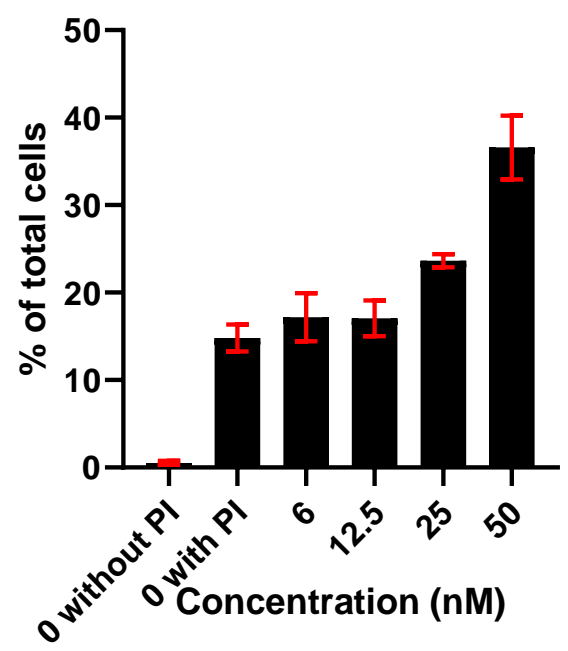

Zampanolide Dead Cells

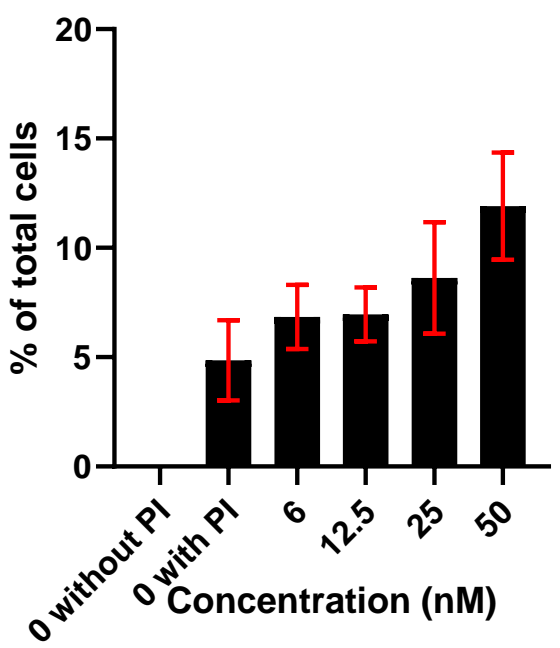

Figure 18. Zampanolide treatment increased percentage of affected and dead A549 cells in a concentration dependent manner.

Concentration of drug treatment was plotted on the $x$-axis. The percentage of total cells in the analysed sample is plotted on the y-axis. 20,000 cells per drug treatment were analysed by flow cytometry with the PEA channel. Results were acquired by flow cytometry. Error bars = SEM; Controls with and without PI were made to assist with gating. 
Doxorubicin was tested at a range of concentrations between 62 to $1 \mu \mathrm{M}$. The cytotoxicity assay (figure 19), showed when concentration of the drug increased so did the percentage of dead and affected cells. However, $1 \mu \mathrm{M}$ reversed this pattern. The percentage of dead cells for the $1 \mu \mathrm{M}$ treatment slightly reduced, compared to the $500 \mathrm{nM}$. It is hypothesised that at this concentration the cells were entirely obliterated into debris, and could not be recognised by the flow cytometer.

Doxorubicin Affected Cells

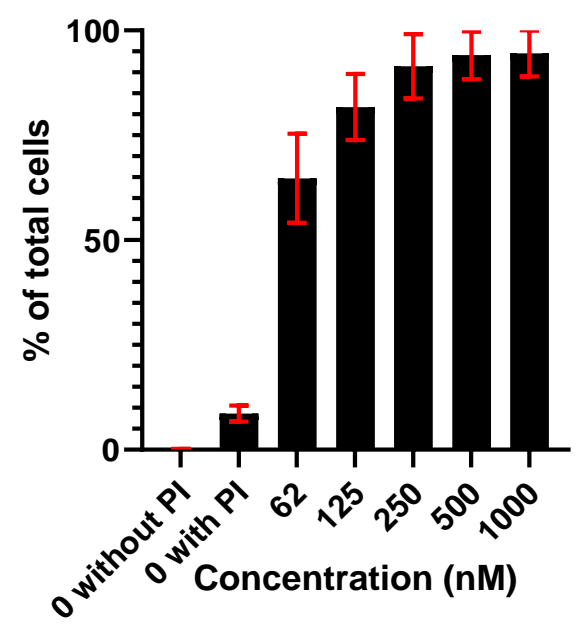

Doxorubicin Dead Cells

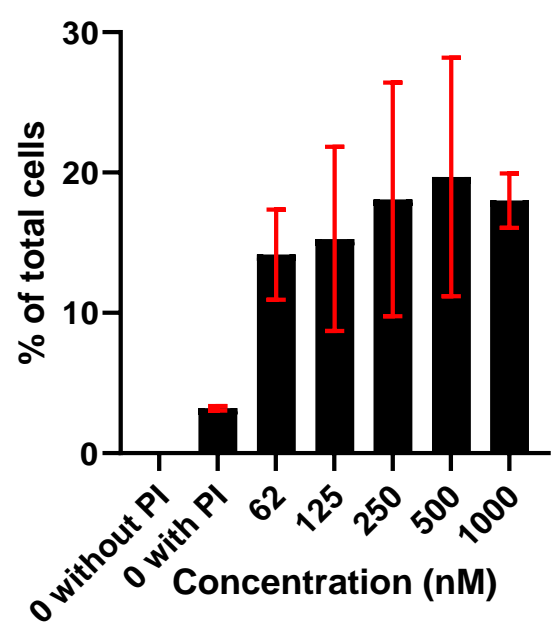

Figure 19. Doxorubicin treatment increased percentage of affected and dead A549 cells in a concentration dependent manner.

Concentration of drug treatment was plotted on the $x$-axis. The percentage of total cells in the analysed sample is plotted on the $y$-axis. 20,000 cells per drug treatment were analysed by flow cytometry with the PEA channel. Results were acquired by flow cytometry. Error bars = SEM; Controls with and without PI were made to assist with gating. 
For BEZ235, a range of concentrations between 62 to $1 \mu \mathrm{M}$ was used. BEZ235 showed when the concentration increased, the percentage of affected cells also increased (figure 20). The percentage of dead cells, however, increased and stagnated from 62 to $250 \mathrm{nM}$ and subsequently decreased for 500 and $1 \mu \mathrm{M}$. The reason for this could be similar to the reason doxorubicin had a decrease in percentage of dead cells at $1 \mu \mathrm{M}$.

BEZ235 Affected Cells

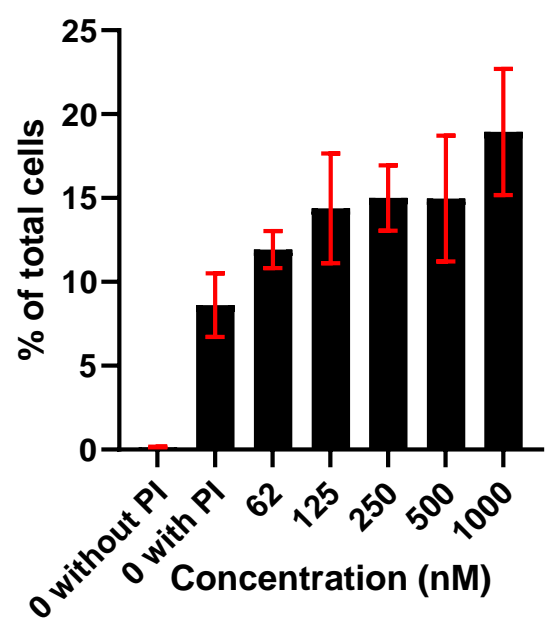

BEZ235 Dead Cells

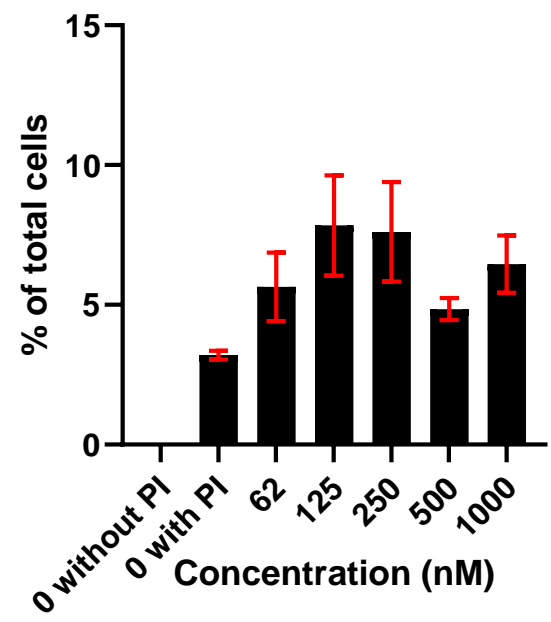

Figure 20. BEZ235 treatment increased percentage of affected and dead A549 cells in a concentration dependent manner.

Concentration of drug treatment was plotted on the $x$-axis. The percentage of total cells in the analysed sample is plotted on the y-axis. 20,000 cells per drug treatment were analysed by flow cytometry with the PEA channel. Results were acquired by flow cytometry. Error bars = SEM; Controls with and without PI were made to assist with gating. 
A66 was also tested at concentrations between 62 and $1 \mu \mathrm{M}$. However, results showed that A66 did not affect or kill the cells in a positive correlation with concentration as seen by previous drugs (figure 21). The percentage of affected and dead cells did not noticeably increase as the concentration increased. The percentage of affected cells may have slightly increased, however, it was indeterminable from this data.

A66 Affected Cells

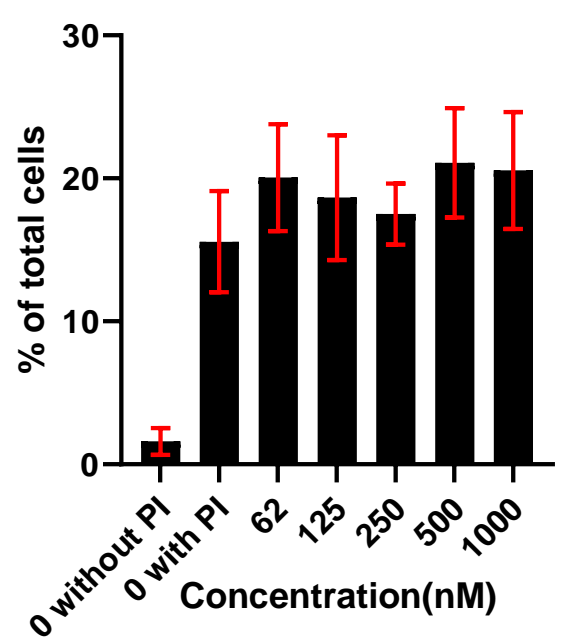

A66 Dead Cells

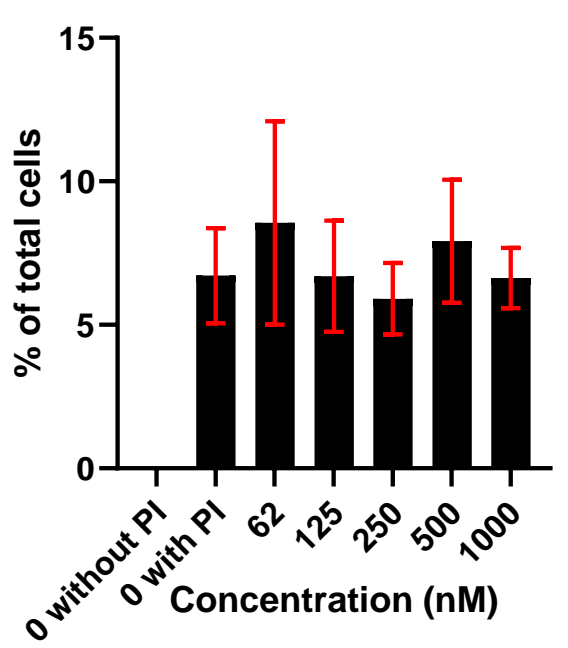

Figure 21. A66 treatment did not affect and or kill A549 cells.

Concentration of drug treatment was plotted on the $x$-axis. The percentage of total cells in the analysed sample is plotted on the y-axis. 20,000 cells per drug treatment were analysed by flow cytometry with the PEA channel. Results were acquired by flow cytometry. Error bars = SEM; Controls with and without PI were made to assist with gating. 
Temozolomide was tested at a range of concentrations between 312 and $5000 \mathrm{nM}$. This data suggested the drug was also inactive, and exemplified how drugs don't necessarily comply with the ' $10 \mu \mathrm{M}$ ' bioactivity rule. As shown in figure 22, there was no change in the percentage of cells affected or killed by all treatments, which deemed it not active.
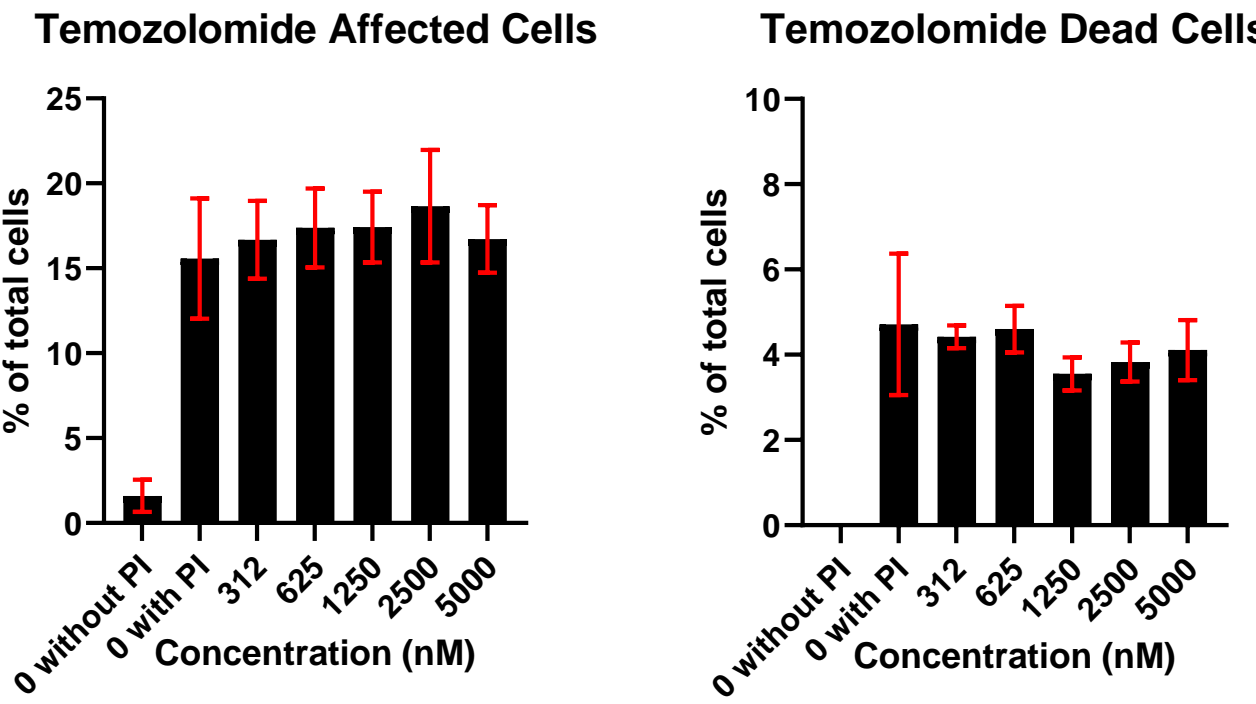

Figure 22. Temozolomide treatment did not affect and or kill A549 cells.

Concentration of drug treatment was plotted on the $x$-axis. The percentage of total cells in the analysed sample is plotted on the y-axis. 20,000 cells per drug treatment were analysed by flow cytometry with the PEA channel. Results were acquired by flow cytometry. Error bars = SEM; Controls with and without PI were made to assist with gating.

\subsubsection{Morphology}

Basic morphological data can be extracted from the same flow cytometry data obtained for the cytotoxicity assay. The parameters include forward scatter, which denotes cell size, and side scatter, which denotes cell granularity. The data were left intentionally ungated to capture the full effect of the drug on the cell population. This data was analysed to further assist addressing the issues found with standardisation of concentration for morphological cell profiling. Specifically, these results attempt to preliminarily understand whether morphological changes still occur when cytotoxic effects are low; that is, a compound gives a signature change corresponding to its bioactive effect. In doing so, it will give further insight into an appropriate Goldilocks concentration for each drug to be used for image-based 
morphological cell profiling assay, and provide certainty that the image-based morphology results are a reflection of the drugs bioactivity. Additionally, drugs within the same class were compared to see if similar activity gave similar morphological effects.

Treatment with Taxol showed morphological changes at all concentrations compared to the control (figure 23). The median of cell size is larger for all Taxol treated samples than untreated. It also increased in variation. In the $500 \mathrm{nM}$ and $1 \mu \mathrm{M}$ treatments, there was a peak with very low forward scatter (figure $23(A)$ ). This indicates debris and reflects the cytotoxicity. The median of cell granularity was larger for all Taxol treated samples than the control. It also increased in variation. A peak with low side scatter was also seen. This again is debris.
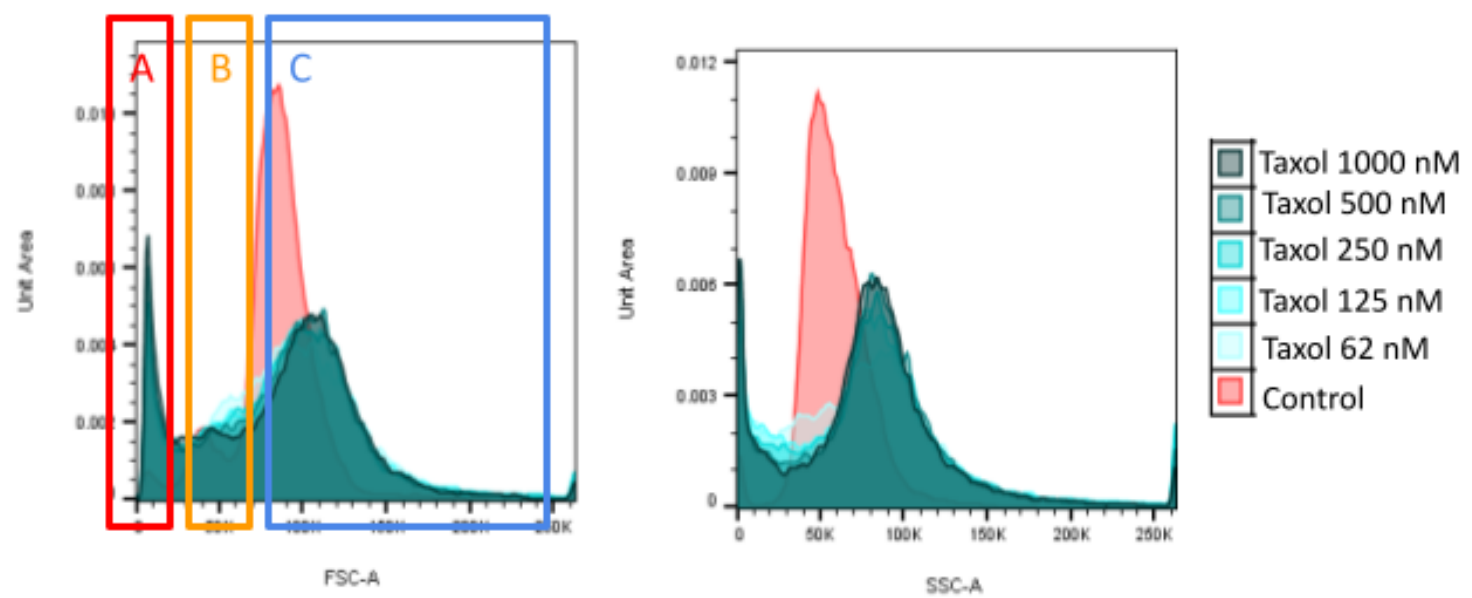

Figure 23. Taxol treatment affected forward scatter (FSC) and cell granularity (SSC).

Results were acquired by flow cytometry synonymous with cytotoxicity data. A (red)= cellular debris, $B$ $($ orange $)=$ small cells with a high proportion of dead cells, $C($ blue $)=$ viable and large cells. Legend labels with treatment concentration are on the right. Control is shown in red and the tone of blue for the treatment samples gets darker as concentration increases. 
Treatment with zampanolide showed morphology changed in parallel to an increase in concentration (figure 24). As the treatment concentration increased so did cell size and granularity. The variation of both parameters also increased as treatment concentration did. This shift was more subtle than Taxol, and not as sudden. This is most likely due to the differences between the range in concentrations tested with respect to its actual bioactive concentration. That is, zampanolide samples were treated at concentrations below and just above its bioactive concentration, whereas all Taxol samples were treated at concentrations above its bioactive concentration. Regardless, morphological changes can be seen in both the 25 and $50 \mathrm{nM}$ treatments suggesting the drug is bioactive. An increase in cellular debris is also seen at 25 and $50 \mathrm{nM}$ treatments.

Interestingly, the overall shift in pattern to morphological changes occurring at bioactive concentrations with both microtubule stabilisers is the same, that is, increased cell size and granularity, as well as increased variation for both parameters.
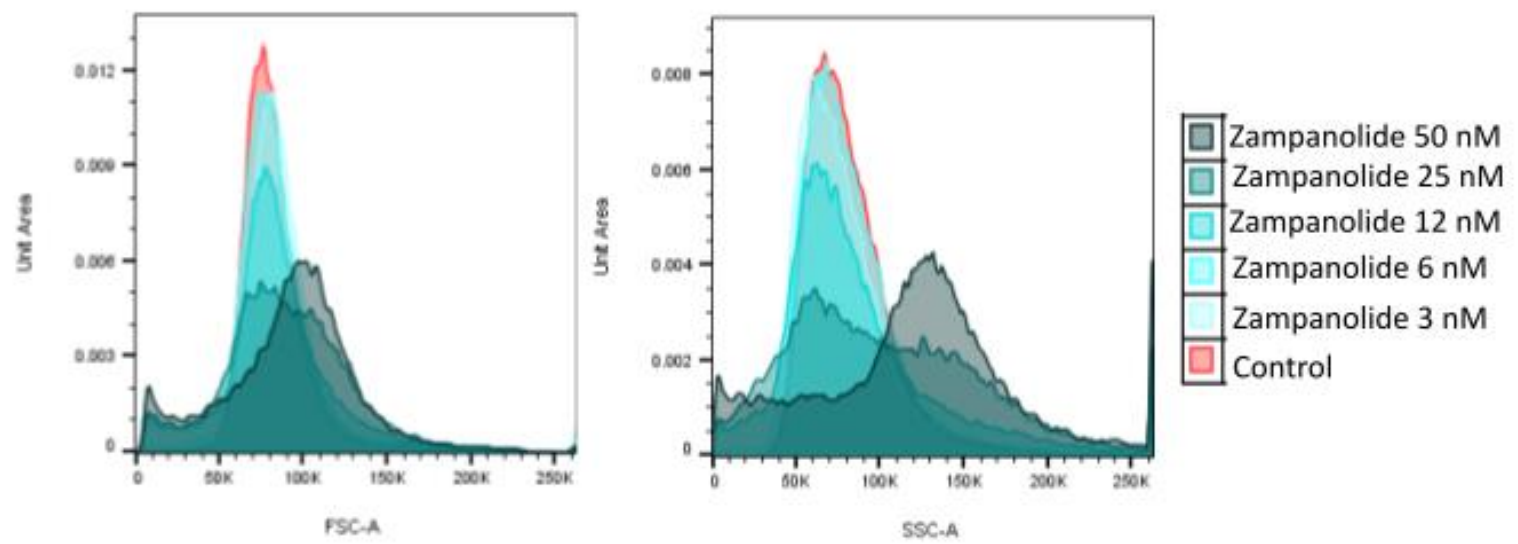

Figure 24. Zampanolide treatment affected forward scatter (FSC) and cell granularity (SSC).

Results were acquired by flow cytometry synonymous with cytotoxicity data. Legend labels with treatment concentration are on the right. Control is shown in red and the tone of blue for the treatment samples gets darker as concentration increases. 
Doxorubicin showed varying changes in morphology as concentration increased (figure 25). Compared to the control, all doxorubicin treated samples changed. At the lowest concentration, $62 \mathrm{nM}$, cell size was the largest out of all samples. Thereafter, cell size decreased as concentration increased. At $1 \mu \mathrm{M}$ median cell size was smaller than in the control and half that of $62 \mathrm{nM}$. This is indicative of a large proportion of cell death as depicted by the orange box in figure $23(B)$. Cell granularity did not follow this relationship. $62 \mathrm{nM}$ was more granular than the control. The largest cell granularity concentration was 125 $\mathrm{nM}$, and thereafter it decreased slightly. At $1 \mu \mathrm{M}$ cell granularity decreased the most. However, unlike the decrease in forward scatter, the reduction in side scatter at $1 \mu \mathrm{M}$ is not indicative of cell death, as shown in appendix 3 (Cii), but rather just a reduction in cell granularity. At the $1 \mu \mathrm{M}$ treatment concentration, a second peak is present in both flow histograms with low cell size and granularity. This is identical to the peaks seen in the Taxol samples at $500 \mathrm{nM}$ and $1 \mu \mathrm{M}$, and once again most likely correlates to cellular debris from obliterated dead cells as a result from a too cytotoxic concentration used.
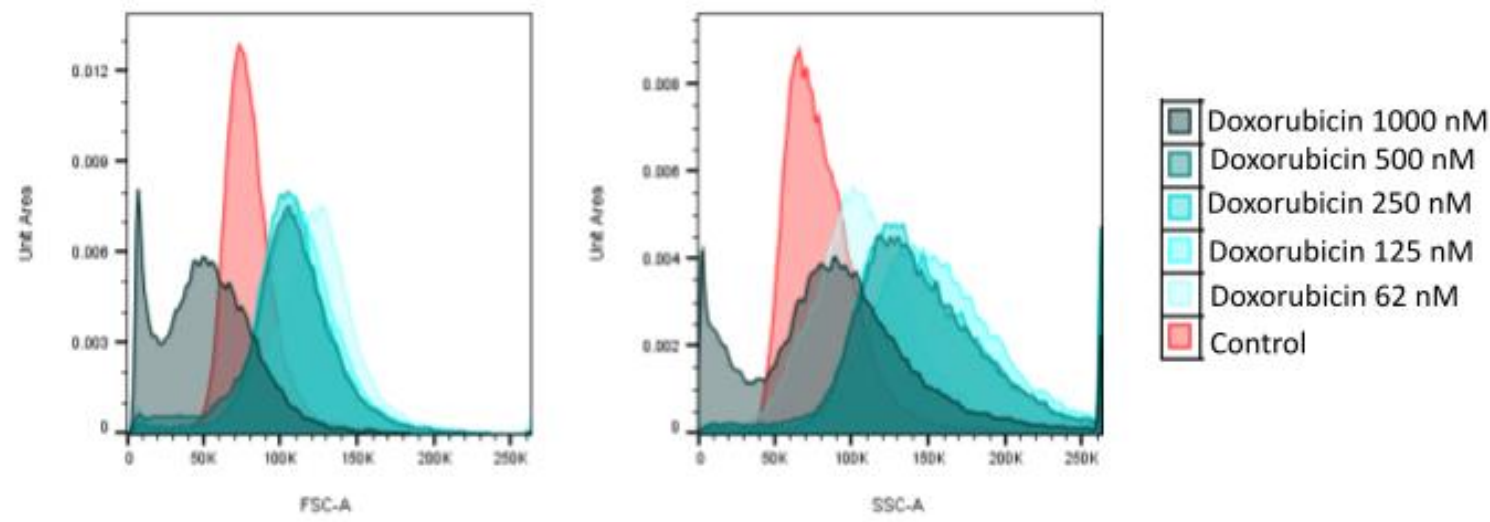

Figure 25. Doxorubicin treatment affected forward scatter (FSC) and cell granularity (SSC).

Results were acquired by flow cytometry synonymous with cytotoxicity data. Legend labels with treatment concentration are on the right. Control is shown in red and the tone of blue for the treatment samples gets darker as concentration increases. 
BEZ235 showed no difference in cell size for treated samples compared to the control (figure 26); as concentration increased there was no change in cell size. Cell granularity, however, did change. As concentration of BEZ235 increased the variation in cell granularity increased, skewing towards a larger granularity. This increase in variation is most likely because the drug increased the granularity within the individual cells by different amounts depending on the stage of the cell cycle the cells were in. Due to this change in side scatter BEZ235 displayed signs of bioactivity. Further, a second peak was present in both flow histograms with low cell size and granularity as concentration increased. This once again is consistent with cellular debris and thus illustrates the cytotoxic effects of BEZ235.
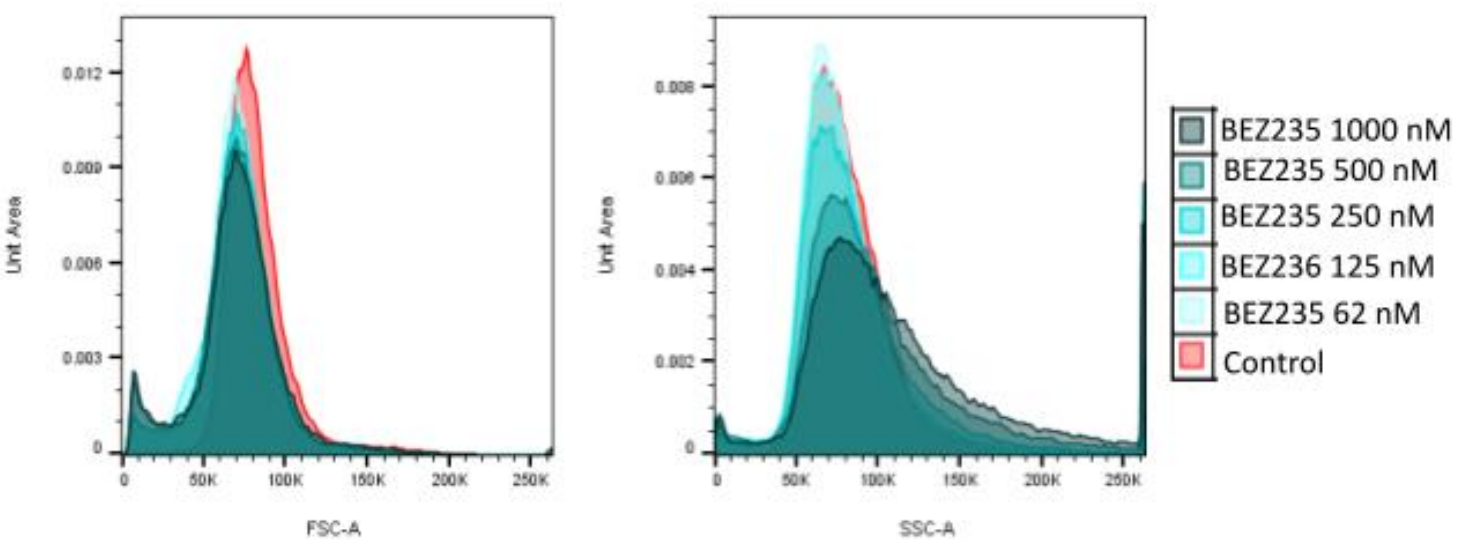

Figure 26. BEZ235 treatment affected forward scatter (FSC) and cell granularity (SSC).

Results were acquired by flow cytometry synonymous with cytotoxicity data. Legend labels with treatment concentration are on the right. Control is shown in red and the tone of blue for the treatment samples gets darker as concentration increases.

A66 showed no significant change in morphology compared to the control (figure 27).

Compared with the cytotoxicity results, which showed not a large amount of toxicity and the cell cycle results which showed minimal changes to cell cycle phases, it is likely no morphological change is observed due to a bioactive concentration not being tested. Concentrations higher than $1 \mu \mathrm{M}$ would need to be tested in order to confirm this. 

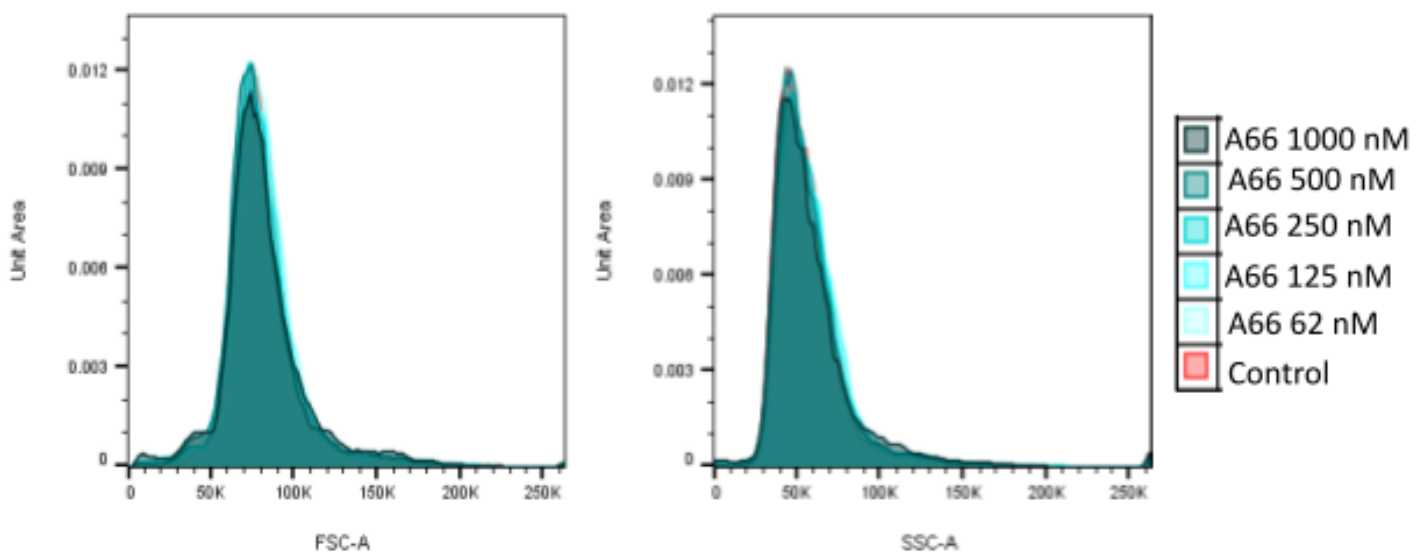

Figure 27. A66 treatment affected forward scatter (FSC) and cell granularity (SSC).

Results were acquired by flow cytometry synonymous with cytotoxicity data. Legend labels with treatment concentration are on the right. Control is shown in red and the tone of blue for the treatment samples gets darker as concentration increases.

Temozolomide also showed no significant change in morphology compared to the control (figure 28). This was expected due to a bioactive concentration of the drug purposely not being tested.
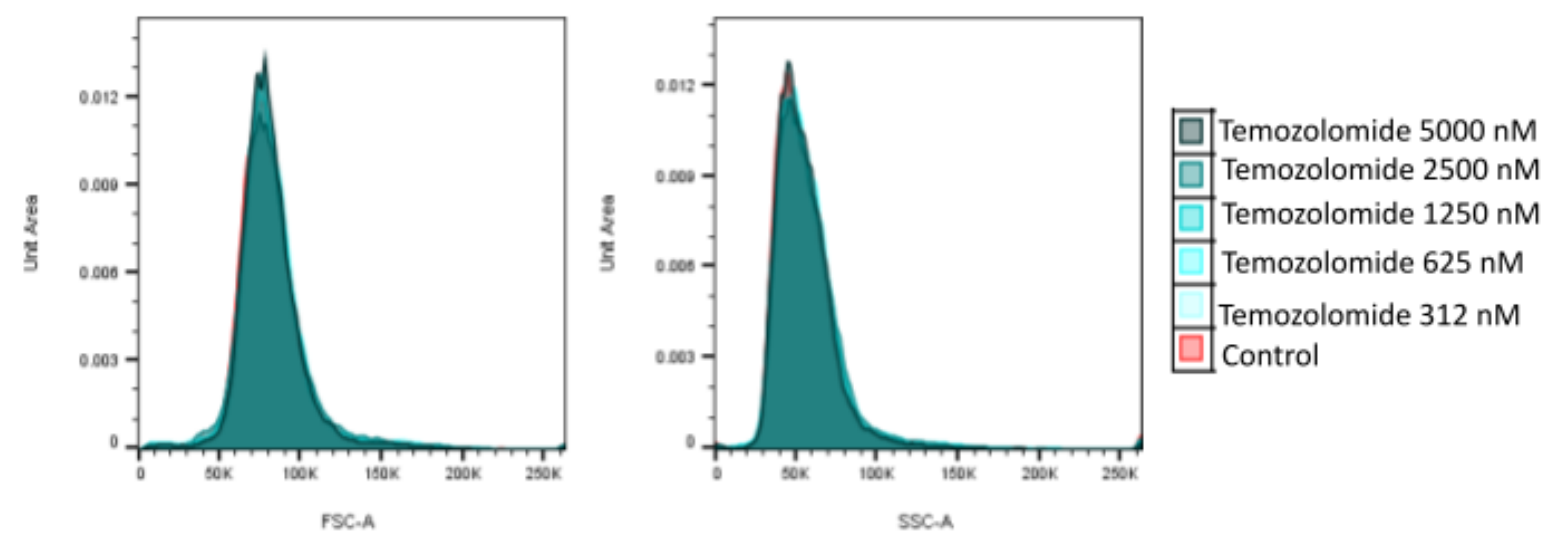

Figure 28. Temozolomide treatment affected forward scatter (FSC) and cell granularity (SSC).

Results were acquired by flow cytometry synonymous with cytotoxicity data. Legend labels with treatment concentration are on the right. Control is shown in red and the tone of blue for the treatment samples gets darker as concentration increases. 


\subsection{Discussion}

The flow cytometry cytotoxicity assay demonstrated the issues with standardisation of compound concentrations for previous morphological cell profiling assays. Specifically, the flow cytometry assay is a semi-quantitative analysis which showed each drug has drastically different bioactive concentrations (figures 17 -22). For example, zampanolide was bioactive at $25 \mathrm{nM}$ (figure 18), but temozolomide showed no bioactivity at $5 \mu \mathrm{M}$ (figure 22). The drugs also showed toxic effects at different concentrations, even with drugs of similar targets. For example, zampanolide showed notable toxic effects on A549 cells at $50 \mathrm{nM}$ (figure 18), but Taxol, the other microtubule stabiliser, showed no notable toxic effects until $500 \mathrm{nM}$ (figure 17). If the standardisation of compound concentrations method used in Svenningsen \& Poulsen (2019) was used here, zampanolide, doxorubicin and BEZ235 would have presented a high proportion of cell morphology representative of cell death for all three concentrations (100 nM, $1 \mu \mathrm{M}$ and $10 \mu \mathrm{M}$ ) (figures 18 - 20). Taxol, however, would have presented morphological changes representative of its microtubule stabilising activity at 100 $\mathrm{nM}$, but cytotoxic morphology at $1 \mu \mathrm{M}$ and $10 \mu \mathrm{M}$ (figure 17). A66 would have presented no activity at all at $100 \mathrm{nM}$, and some bioactive effect at $1 \mu \mathrm{M}$ (figure 21); and temozolomide would have shown no bioactive morphological change at any of the concentrations used (figure 22). Together it highlights the potential erroneous nature of concentration standardisation for MCP assays.

This flow cytometry cytotoxicity and morphology data also provided information as to what concentration each drug should be ideally used at to illustrate activity. For all drugs that should have shown bioactivity below $10 \mu \mathrm{M}$, that is, all except temozolomide, the assay accurately defined a bioactive concentration with limited cytotoxic effects for four out of five exemplar drugs - all except A66. For example, using this protocol, it can be deduced that zampanolide started to become active towards A549 cells by $25 \mathrm{nM}$, but notably toxic at 50 $\mathrm{nM}$ (figure 18). In this study, a notable difference is defined by analysis of the error, as assessed by the standard error of the mean (SEM). If the SEM between treatment and control do not overlap (as denoted by the error bars), it is a notable difference, and if they do, it is not. This does not imply, or require, statistical significance - no claim is being made.

Interestingly, the changes in the morphology data paralleled the cytotoxicity data for zampanolide treatment (figure 24). Changes to cell size and granularity occurred at the same concentrations zampanolide was bioactive at, $25 \mathrm{nM}$ and $50 \mathrm{nM}$. This data collectively 
suggests a zampanolide concentration of $25 \mathrm{nM}$ would be best to image cells at for an MCP. This is because the drug would have elicited a morphological change representative of the drugs bioactivity, and minimal cytotoxic morphological change would be seen.

Taxol was active at all concentrations tested between $62 \mathrm{nM}$ to $1 \mu \mathrm{M}$, but the cytotoxicity subtly increased in a concentration dependent manner (figure 17). Just like zampanolide, the Taxol morphology data is consistent with the cytotoxicity assay data as all bioactive treatments changed morphology (figure 23). At $250 \mathrm{nM}$ the percentage of affected cells is notable, but cell death was not. At $500 \mathrm{nM}$, however, the standard error mean (SEM) bars between the treatment and the control no longer overlap. From this, it was concluded a notable cytotoxic effect was seen at $500 \mathrm{nM}$. Thus, an appropriate Taxol concentration to use for an MCP would be at most the next lowest concentration tested in this cytotoxicity assay, that is, $250 \mathrm{nM}$.

Doxorubicin was effective and cytotoxic at all concentrations tested (figure 19). In parallel, the morphology changed at all the doxorubicin concentrations used (figure 25). From this, concentrations lower than $62 \mathrm{nM}$ would need to be tested to confirm an appropriate concentration for a MCP. Interestingly, the $1 \mu \mathrm{M}$ sample is an anomaly in both the cytotoxicity and morphology data. In the morphology data this can be seen most clearly. Instead of the cell size increasing, consistent with the concentration dependent pattern the other concentrations adhere to, the cell size remarkably decreased at $1 \mu \mathrm{M}$. Within this sample's results there are additional peaks which denote objects that are miniscule in size and granularity. These two patterns suggest $1 \mu \mathrm{M}$ is highly toxic as it corresponds to a high proportion of dead cells and cellular debris respectively.

BEZ235 treatment caused results similar to doxorubicin treatment (figure 20). All concentrations tested affected cells or caused cell death. However, unlike doxorubicin, it did not elicit the same obvious morphological change (figure 26). Instead, only cell granularity increased in a concentration dependent manner, and no changes occurred to cell size. Concentrations lower than $62 \mathrm{nM}$ would need to be tested to confirm an appropriate concentration for a MCP to reduce the morphological features of cell death being imaged. Curiously, the percentage of dead cells decreased at $500 \mathrm{nM}$ and $1 \mu \mathrm{M}$. This goes against the positive concentration-dependent relationship expected with a drug cytotoxicity assay. However, there is a second peak of small objects identified by the flow cytometer. This is cognizant of dead cell debris. It is more reasonable to suggest at these BEZ235 
concentrations the cells are completely obliterated and registered as debris rather than cells by the flow cytometry gating strategy (appendix 2). This is consistent with the morphology data (figures $20 \& 26$ ). Additionally, this aligns with the hypothesis suggested by doxorubicin treatment's morphology data (figure 25), that at concentrations too cytotoxic, cells become debris.

A66 and temozolomide treatments both did not elicit a toxic or morphological effect on cells (figures $21 \& 22$ ). However, suggested by the cell cycle data with different A66 treatments (figure 13), the drug should have a some bioactive effect on cells at $1 \mu \mathrm{M}$. Further testing above $1 \mu \mathrm{M}$ would be necessary in order to discover if the compound is active and which concentration would be best suited for an MCP.

Based on what is learnt from the exemplar drug flow cytometry cytotoxicity and morphology results, the definition and protocol to realise a Goldilocks concentration for a MCP can be rationalised.

The rules for a Goldilocks concentration for an MCP must meet the following criteria:

1. There is a notable difference in the percentage of affected cells, or FSC or SSC changes

2. There is no notable difference in the percentage of dead cells.

Protocol rules to find the Goldilocks concentration of drugs for a morphobase and novel natural product compounds with a flow cytometry cytotoxicity assay is as described below:

1. A549 cells must be plated at $5 \%$ confluency 24 hours before treatment

2. Initial screens of compounds are treated at $1 \mu \mathrm{M}, 500,250$, and $125 \mathrm{nM}$

3. Experiment is done in technical duplicate and biological triplicate to ensure substantial and consistent SEM

4. If there is no bioactivity seen at $1 \mu \mathrm{M}$, retest the compound at $10 \mu \mathrm{M}$

5. If the compound is too cytotoxic at $125 \mathrm{nM}$, retest at $62,31,16,8$ and $4 \mathrm{nM}$.

However, as with any assay, there are some disadvantages with this protocol. 
First, the concentration range chosen in step 2 of the protocol may not be wide enough to encompass the Goldilocks concentration. However, this was deemed the best range from the exemplar drug treatment results. This was because within natural product discovery, the amount of compound isolated is miniscule, within milligrams. Thus, by limiting the top concentration initially tested to $1 \mu \mathrm{M}$, instead of $10 \mu \mathrm{M}$, as carried out by Svenningsen \& Poulsen (2019) and recommended by the Journal of Natural Products Guideline for Authors, it reduces the amount of a novel compound being used and therefore reduces the risk of using it all - which will be needed for the MCP and future experiments. Step 4 was added to safeguard that the cytotoxicity threshold stipulated in Journal of Natural Products Guideline for Authors was adhered to and identify bioactive compounds, such as A66, which are not bioactive at $1 \mu \mathrm{M}$.

The lowest concentration initially tested is $125 \mathrm{nM}$ (Step 2). This was chosen as it optimises the range in which the likelihood of both the compound being active and the Goldilocks concentration being found. To clarify, the hypothesis created was that it is unlikely a novel compound will be as active as the exemplar compounds as they are all well-known active compounds. For example, zampanolide, doxorubicin and BEZ235 would have been too active and cytotoxic at the lowest concentration this protocol initially tests at (step 2). In reality, however, once the MCP assay is established the novel compounds tested may not be as bioactive as these exemplar ones. Thus, in restricting the initial range of concentrations tested from $125 \mathrm{nM}$ to $1 \mu \mathrm{M}$ (step 2), it will not only optimise the laboratory time and resources used, but limits the chance of exhausting the supply of the compound.

Second, this flow cytometry cytotoxicity assay is time intensive. Unlike the concentration standardisation approach in Svenningsen \& Poulsen, additional experiments on flow cytometry must be undertaken before the MCP analysis can begin. However, the protocol to carry out this assay as stated in 2.1.6.2. could be made more automated. The flow cytometer used can run 96-well plates automatically. Therefore, hundreds of samples could be analysed in a day and therefore would speed up data acquisition.

Third, it requires biologists and keen chemists to know how to operate a flow cytometer; if not, learning how is another labour and time intensive process. Despite the cytometer not being particularly hard to operate once learnt, it should not be underestimated when assessing the feasibility of an assay. 
Fourth, the assay may still reject novel compounds with clinical potential. This is shown by temozolomide treatment results whereby no activity was seen at $5 \mu \mathrm{M}$ yet literature states it is active in vitro at concentrations $500 \mathrm{mM}$ and above. ${ }^{78}$ Admittedly, temozolomide is not an ideal drug in vitro, but has activity in vivo.

Another element to consider is some may argue the flow cytometry cytotoxicity and morphology data could be used exclusively as a quantitative non-imaged-based morphological cell profiling assay. Thus, a future direction of this work could be to assess this in more detail. However, an image-based morphological cell profiling assay has the potential to analyse many more morphological parameters than flow cytometry, such as cell roundness and cellular protrusions, as well as specific cellular components and organelles, such as the mitochondria and cytoskeleton. This, in theory, should widen the scope for what the assay can be used for in the future, including the potential to define a mechanism of action.

\subsection{Conclusion}

Using a flow cytometry assay to determine a Goldilocks concentration for compounds is a more relevant approach to determine the concentration of treatment for an MCP than concentration standardisation. It is time intensive, but provides more biologically relevant and accurate information about the drug's bioactivity for an MCP assay, and for that reason it is feasible, even necessary for a researcher to carry out this assay before following through with MCP analysis. The fact it also provides preliminary morphological data is crucial to the benefit of it. 


\section{Chapter 4: Establishing the foundations of an imaged-based MCP at Te Herenga}

Waka 


\subsection{Introduction}

An image-based approach would give more morphological information about the effect of a drug than a flow cytometry approach. This is why this chapter aims to investigate the feasibility of an image-based approach to a MCP assay at Te Herenga Waka.

Flow cytometry analyses cells as individual, floating objects and can measure two basic morphological parameters, cell size and granularity. While this is very useful, as shown in the previous chapter, there are far more morphological characteristics to be seen and measured when cells are stretched out on a flat surface. For example, most information on the shape of the cells - how round or elliptical, and the number and length of protrusions are lost in flow cytometry. Further, the morphology of organelles such the nucleus, and intracellular structures like actin filaments give important information as to cell state, and could also be measured and analysed.

An image-based approach attempts to quantify what the human eye can see. To set up an image-based MCP assay two features are vital. First, a way to capture images of lots of cells with a microscope. Second, a way to quantify and analyse the cell morphology from the image. Fortunately, these two steps are possible in a small chemical biology laboratory, such as the one at Te Herenga Waka. This is because most small chemical biology laboratories have access to a microscope, and the software needed for image analysis is free-access and easily downloaded from the internet.

The software primarily used to identify and quantify cellular components for MCP is CellProfiler. This software is free-access and easily downloaded from the internet. It has extensive morphological parameters it can measure, such as cell roundness, ellipticity, and protrusion length. For this reason, it will be used to carry out MCP.

\subsection{Aims}

1. Establish whether different drugs cause different morphological changes determinable by eye

2. Establish a CellProfiler pipeline to generate morphological data from images

3. Use a CellProfiler pipeline to attempt to establish the foundations of an image-based $\mathrm{MCP}$ at Te Herenga Waka 


\subsection{Results}

\subsubsection{Image acquisition}

\subsubsection{1. $\quad$ Morphology changes in drug treated cells}

To deepen the understanding of the morphological changes seen by flow cytometry, treated cells were imaged by brightfield microscopy to observe the changes by eye. A549 cells were treated with three of the exemplary drugs for 48 hours, based on the data from chapter 3 and then imaged. A variety of cell morphology responses to the drugs were observed. In figure 29, as zampanolide concentration increased the cells that maintained enough viability to still adhere to the plate became rounder and larger. Similarly, as the doxorubicin concentration increased, the adhered cells became even larger than zampanolide treatment (figure 30). On top of this, the nuclei became larger and more elongated. In contrast, as BEZ235 concentration increased, cells became more clumped together and lost their protrusions

(figure 31). These images highlighted how different drugs with different mechanisms cause different morphological changes to cells.

\section{Zampanolide}

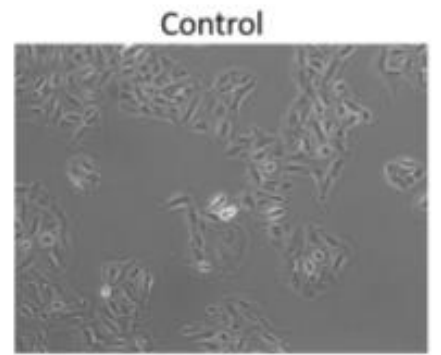

$25 \mathrm{nM}$

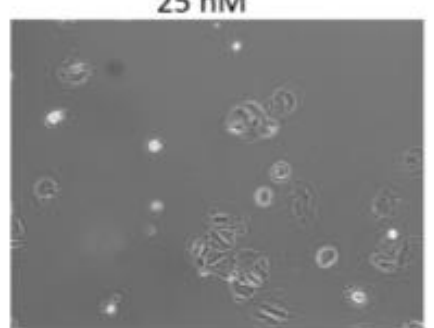

$6.25 \mathrm{nM}$

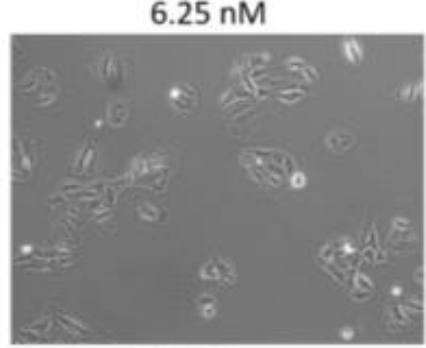

$50 \mathrm{nM}$

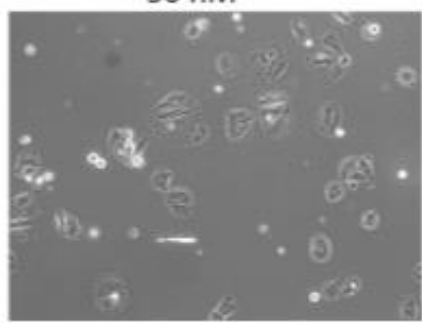

$12.5 \mathrm{nM}$

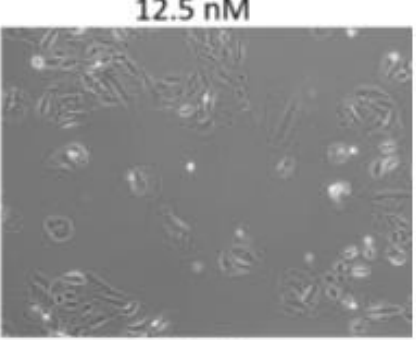

$100 \mathrm{nM}$

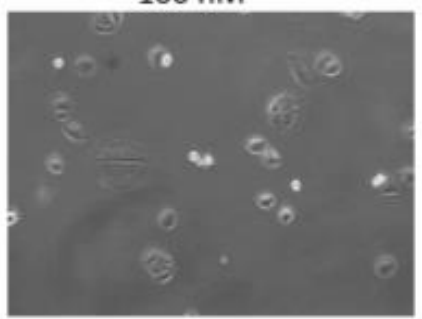

Figure 29. Zampanolide treatment made cells larger and rounder in brightfield.

Images were taken on phase-contrast inverted microscope with a 10x magnification lens of A549 cells following a $48 \mathrm{hr}$ treatment incubation time. Images are representative of treatment conditions from a 6-well plate, $n=4$. 


\section{Doxorubicin}

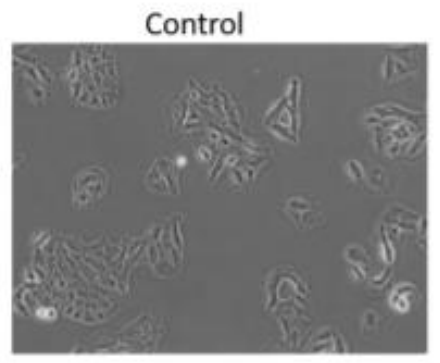

$250 \mathrm{nM}$

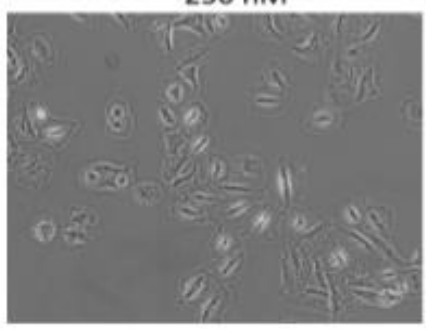

$62.5 \mathrm{nM}$

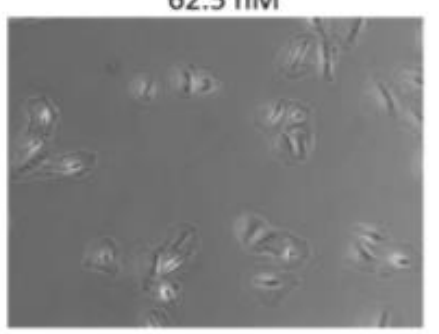

$500 \mathrm{nM}$

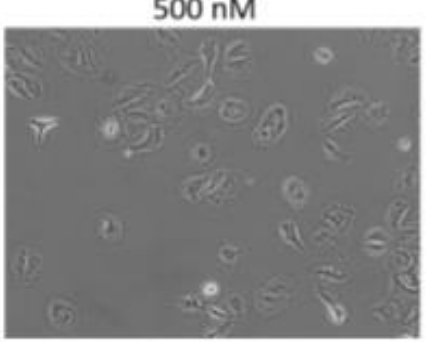

$125 \mathrm{nM}$

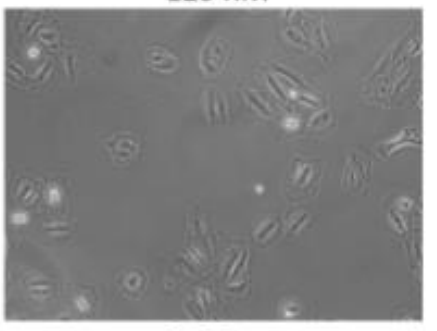

$1 \mathrm{uM}$

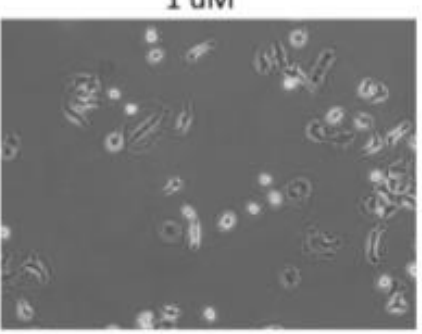

Figure 30. Doxorubicin treatment made cells larger in brightfield.

Images were taken on phase-contrast inverted microscope with a 10x magnification lens of A549 cells following a $48 \mathrm{hr}$ treatment incubation time. Images are representative of treatment conditions from a 6-well plate, $n=4$.

\section{BEZ235}

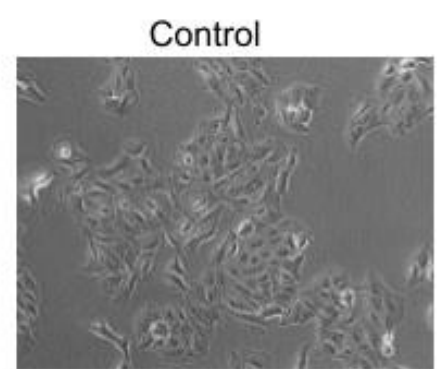

$250 \mathrm{nM}$

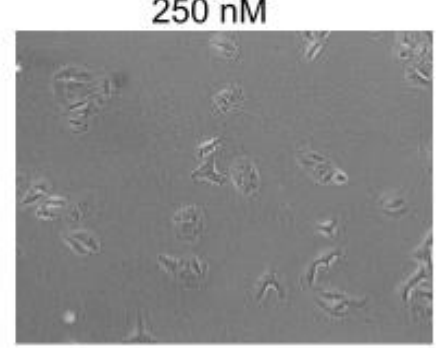

$62.5 \mathrm{nM}$

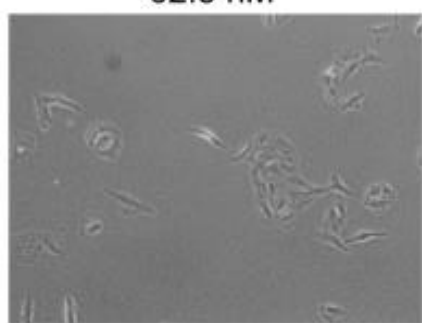

$500 \mathrm{nM}$

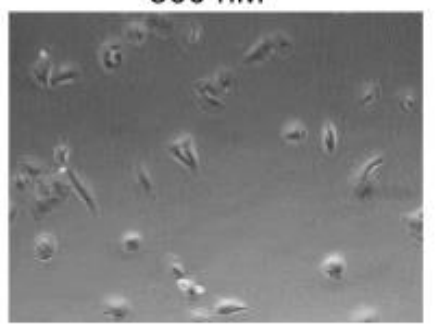

$125 \mathrm{nM}$

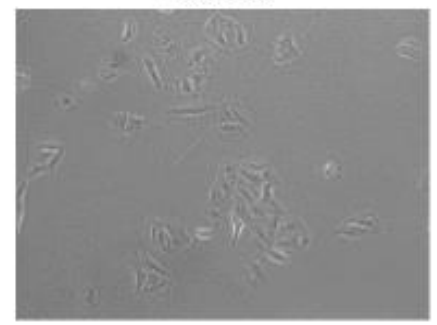

$1 \mathrm{uM}$

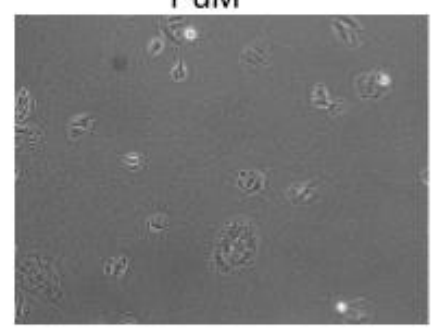

Figure 31. BEZ235 treatment made cells clump together and lose protrusions in brightfield. 
Images were taken on phase-contrast inverted microscope with a 10x magnification lens of A549 cells following a $48 \mathrm{hr}$ treatment incubation time. Images are representative of treatment conditions from a 6-well plate, $n=4$.

\subsubsection{Why brightfield is not enough-CellProfiler needs separate images of cellular compartments}

In order to create an image-based assay, brightfield images cannot be used. This is because although the cytoplasm and nucleus can be seen by eye, software cannot distinguish between the two in order to measure the morphology. Instead the individual components must be fluorescently stained. Additionally, with fluorescence, other intracellular organelles, which cannot be seen by eye, such as actin filaments or mitochondria, can also be observed and measured. Thus, fluorescent stains not only allow for the cell to be identified for morphological analysis, but widen the possibilities of what can be learned about the drugs tested by measuring the morphology of more organelles. In doing so, MCP with an image based approach has the capabilities to see differences that cannot be seen by the human eye with a lab bench microscope.

\subsubsection{Making cells fluorescent}

The fluorescent stains are an essential part to MCP to ensure the most accurate morphological data could be acquired. This is because the fluorescence intensity of the stain is directly used by the CellProfiler to distinguish cellular components for object identification and segmentation. Initially, stains used identified the nucleus and cytoplasm.

As seen in figure $32 \& 33(A)$, DAPI was used to stain the nucleus. This was adequate for nuclei segmentation due to the visible and even saturation fluorescence of the nuclei. Three stains for cytoplasm identification and cell segmentation were tested. They were CFSE (figure 32 \& $33(B)$ ), ActinRed (figure 32 \& $33(C)$ ), and CellMask Deep Red Plasma Membrane (figure $32 \& 33(D)$ ). CFSE binds to cytoplasmic proteins, ActinRed binds to actin filaments, and CellMask Plasma Membrane Far Red binds to the plasma membrane.

Figure $32 \& 33$ show the quality of the stains after images were captured on the highthroughput laser scanning IN Cell Analyser 6500 confocal microscope and the laser scanning Olympus FV3000 confocal microscope respectively. Figure 32 shows that the best stain to use on the IN Cell for cytoplasm segmentation was the CellMask Plasma Membrane Far Red. 
This is because visible and even saturation is necessary for the software to identify the cytoplasm. A comparison between the stains was also carried out with the Olympus FV3000 as an initial image analysis optimisation and analysis protocol was carried out on a few images from this microscope. Figure 33 shows that on the Olympus FV3000 confocal, CellMask Plasma Membrane stain also had satisfactory fluorescence intensity for cytoplasm identification.
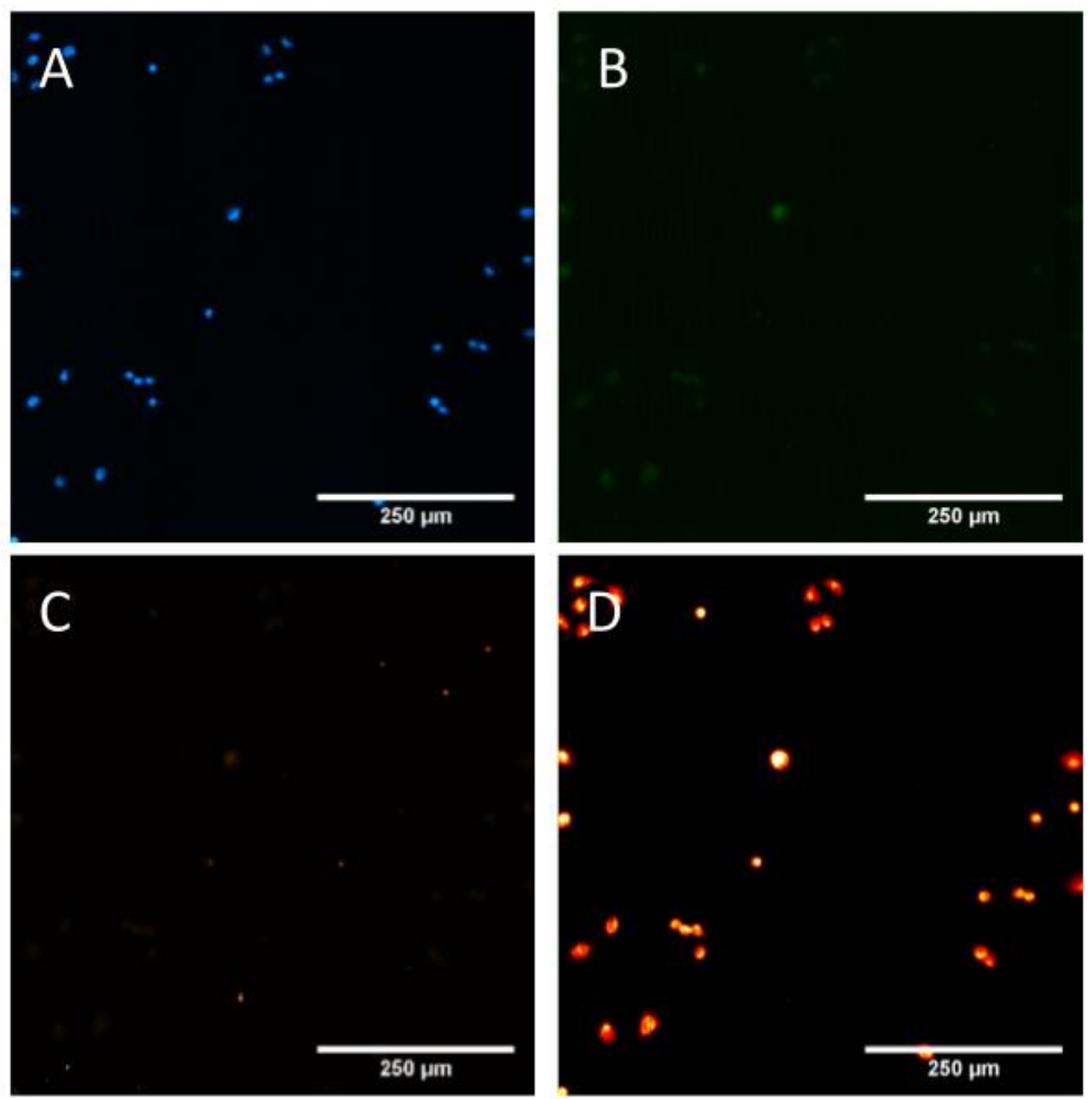

Figure 32. Stained A549 cells imaged on the IN Cell Analyser 6500.

$A=$ nucleus stain, DAPI; $B=$ cytoplasmic protein stain, CFSE; $C=$ actin stain, ActinRed $;$ and D = plasma membrane stain, Cell Mask Deep Red Plasma Membrane. Images are representative from a 6-well plate, $n=$ 4. 

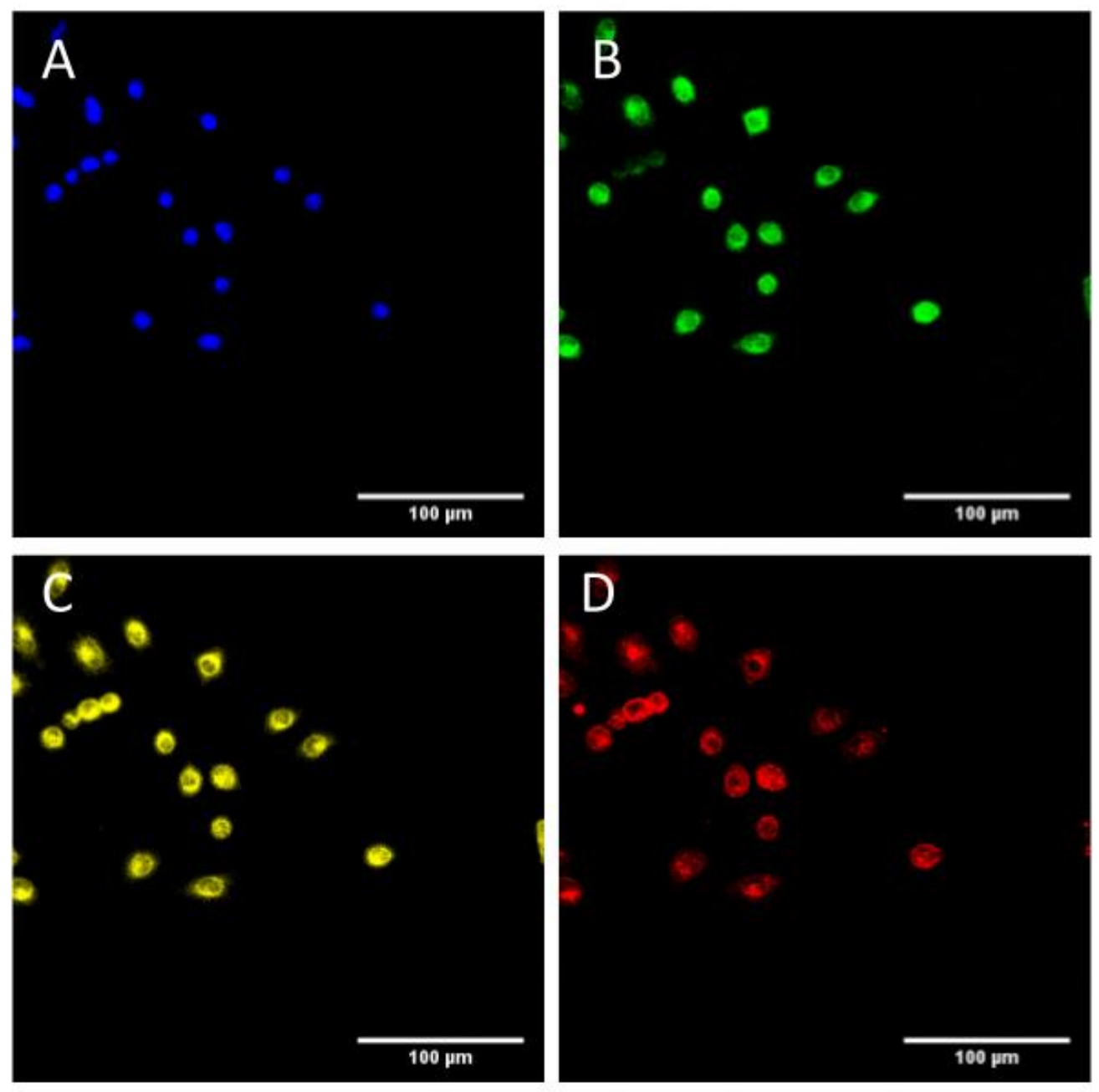

Figure 33. Stained A549 cells imaged on the Olympus FV3000.

$A=$ nucleus stain, DAPI; $B=$ cytoplasmic protein stain, CFSE; $C=$ actin stain, ActinRed $;$ and D = plasma membrane stain, Cell Mask Deep Red Plasma Membrane. Images are representative from a 6-well plate, $n=$ 4.

\subsubsection{Suitability of microscopes for $M C P$}

At Te Herenga Waka there are three microscopes that may have been suitable for image acquisition for MCP. They were the Olympus FV3000, the GE Healthcare/Cytiva IN Cell Analyser 6500 and the Olympus Slide Scanner VS200.

A comparison of the three microscope's abilities can be seen in table 2.

Preliminary data was obtained using the Olympus FV3000, due to its ability to take very good quality images with high resolution. The pixel size resulting from high resolution meant 
that object identification by CellProfiler would be more precise, and up to 8 lasers meant many fluorescent channels and filters could be used simultaneously. This allowed the establishment of cell labelling protocols using multiple fluorophores.

However, image acquisition on the Olympus FV3000 was very time intensive. Each slide was individually imaged, and the microscope had a relatively slow capture time. Due to this, the acquisition of sufficient images for quantitative analysis was not feasible and this microscope could not be used to scale up a MCP assay.

The Slide Scanner VS200 was also trialled. This was designed for high-throughput analysis of multiple slides at once, and had the capacity for imaging up to 7 fluorophores at high magnification and in high resolution. However the image files that were generated were not easily extracted in a format compatible with downstream analysis.

The GE IN Cell Analyser 6500, is a high-throughput microscope with high content capabilities. It has the ability to take hundreds of images across multiple slides within an hour, as well as capture multiple fluorescent cellular stains. This meant in theory, unlike the Olympus FV3000, it has the ability to create the quantity of data required for a MCP assay.

The main issue with this microscope was that the autofocus function was not always accurate and thus produced a lot of defective images. Issues with the defective images were autofluorescent debris and artefacts, over and under saturation, and blur. Examples are shown in

\section{figure 34.}

Table 2. Comparison of the microscopes available to Te Herenga Waka researchers.

*if quality control is applied

\begin{tabular}{|l|l|l|l|l|l|l|}
\hline Microscope & Throughput & Resolution & Content & $\begin{array}{l}\text { Image } \\
\text { Processing }\end{array}$ & $\begin{array}{l}\text { Difficulty of Image } \\
\text { Processing }\end{array}$ & $\begin{array}{l}\text { Robustness } \\
\text { of Data }\end{array}$ \\
\hline Olympus FV3000 & low & high & high & feasible & easy & low \\
\hline $\begin{array}{l}\text { IN Cell Analyser } \\
6500\end{array}$ & high & low & high & feasible & hard & high* \\
\hline $\begin{array}{l}\text { Slide Scanner } \\
\text { VS200 }\end{array}$ & high & high & high & not feasible & - & \\
\hline
\end{tabular}



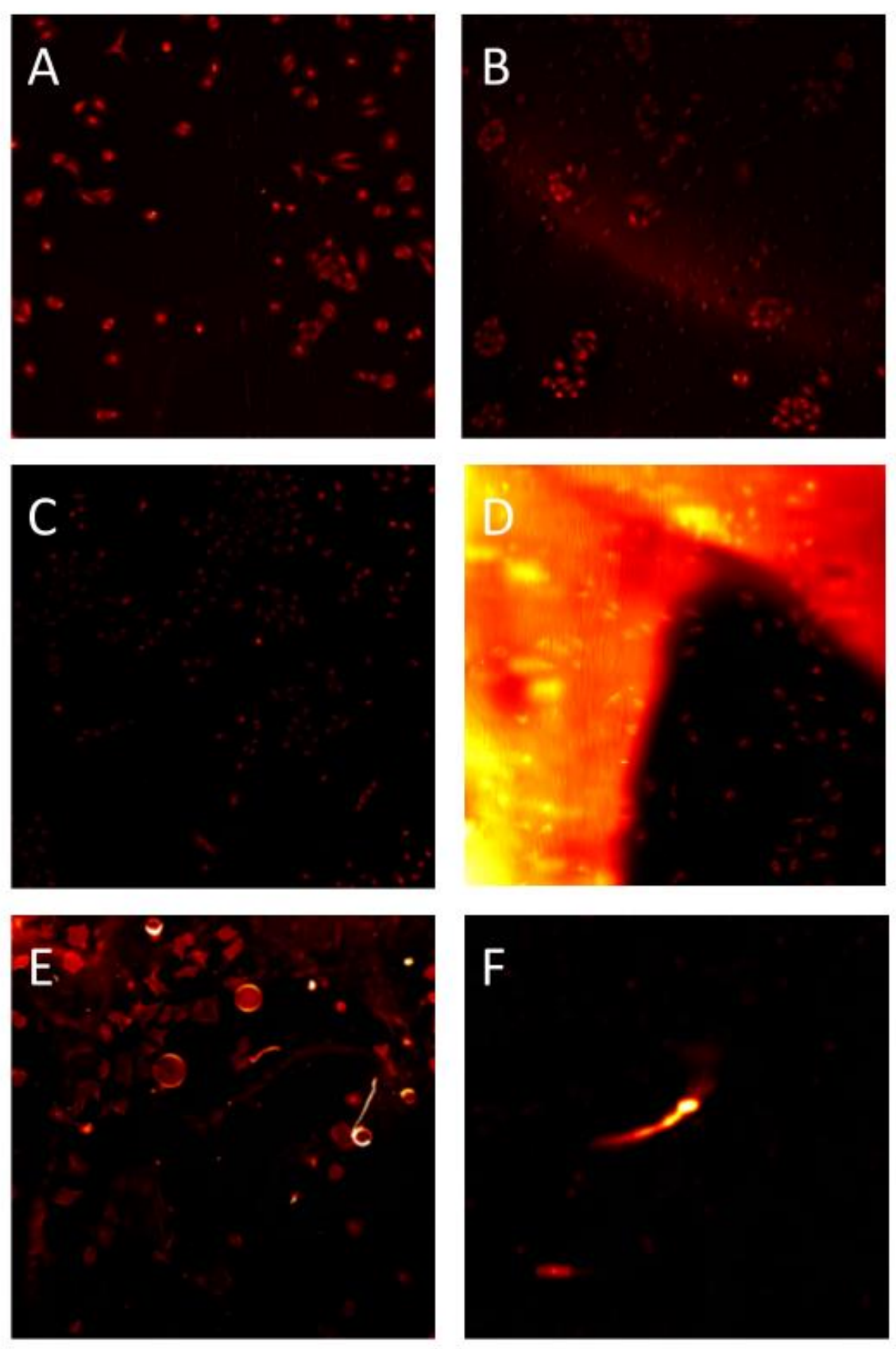

Figure 34. Examples of image aberrations made as a result of the autofocus function on the IN Cell Analyser 6500.

$A=$ blur $; B=$ background illumination; $C=$ under-saturation; $D=$ incorrect focus of over-saturated artefact; $E$ = incorrect focus on auto-fluorescent bubbles; $F$ = incorrect focus of auto-fluorescent debris. 


\subsubsection{Effect of drug treatment on morphology}

Once stains for image acquisition were determined, the ability to visualise the morphological differences was visually assessed. The six drugs which were used in the previous chapter were used to treat A549 cells for imaging, using the 'Goldilocks' concentrations to ensure the morphological differences seen were produced by the drugs' bioactivity. The images captured by the IN Cell Analyser 6500 can be seen in figure 35 - 38 .

The microtubule stabilisers, Taxol and zampanolide changed morphology of the cytoplasm and nuclei. In both treatments the cytoplasm is larger and rounder (figure 35). This is consistent with the zampanolide treated brightfield image, the flow cytometry results in chapter 1 and what is expected from a microtubule stabiliser. Microtubule stabilisers arrest depolymerisation of tubulin filaments, but not polymerisation. This means the cytoskeleton of a cell grows, but cannot retract. Thus, it invokes the cell expansion which can be seen in figure 35 (Aii). The roundness of the cell and lack of protrusions can be accounted for by this cytoskeletal growth as well. The tubulin continues to polymerise in all directions and cannot retract other parts of the cells due to the microtubule stabilisation inhibiting depolymerisation. Thus, a uniform expansion of the cytoskeleton results in this rounder morphology and lack of the individual protrusions. The nuclei are also larger and rounder in the Taxol and zampanolide treated samples compared to the control (figure $35(\mathrm{Ai})$ ). The nuclei from the Taxol treated sample is also notably larger than the zampanolide treated. 

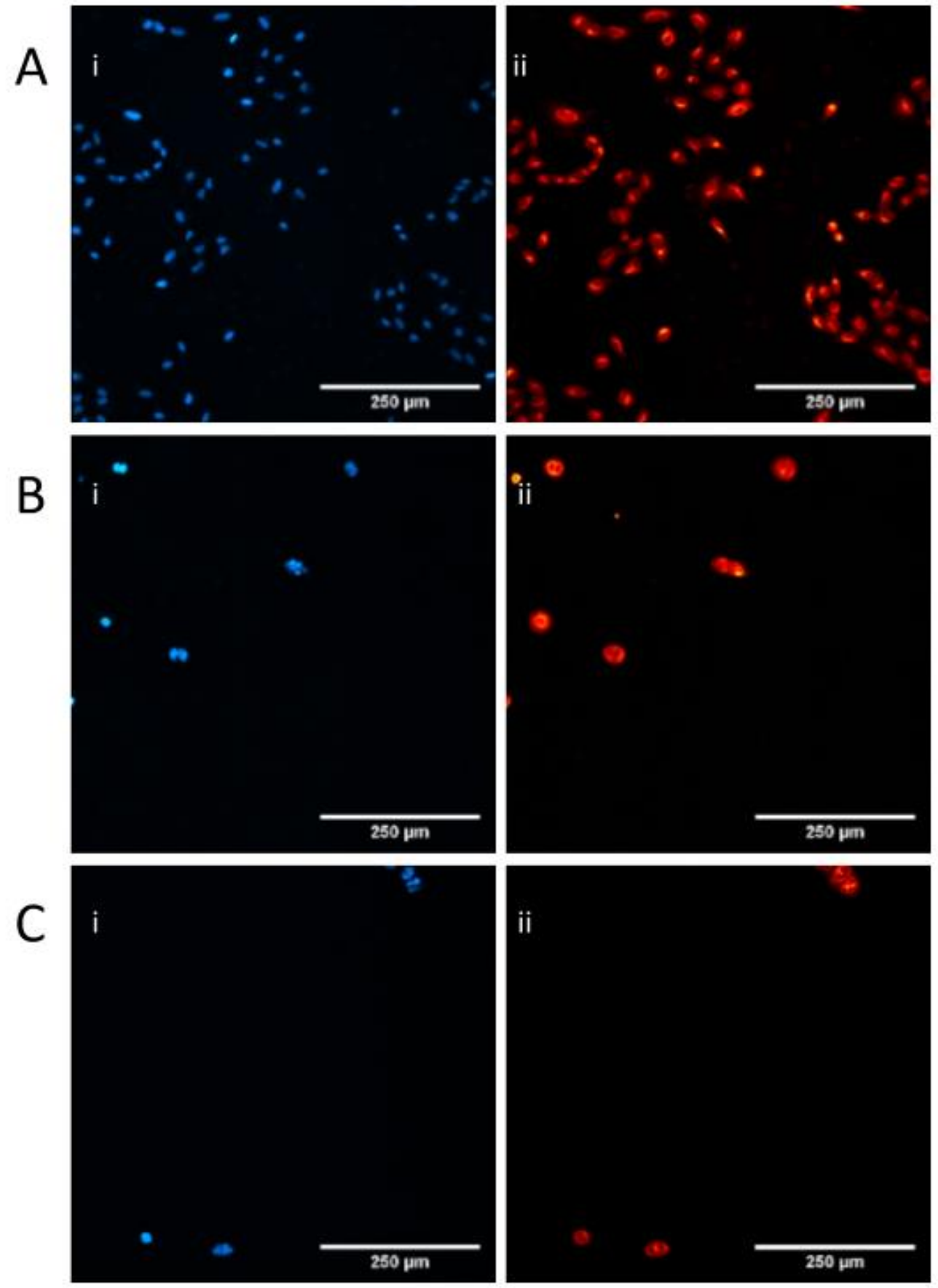

Figure 35. A549 morphology of nucleus and cell after treatment with $250 \mathrm{nM}$ of Taxol and $25 \mathrm{nM}$ of zampanolide.

Treatments: $A=$ Control, $B=$ Taxol, $C=$ zampanolide; Stains: $i=$ DAPI - nucleus stain, $i i=$ Cell Mask Deep Red Plasma Membrane. Images were taken 48 hours after treatment incubation. A549 cells were fixed and adhered to slides, and imaged on the IN Cell Analyser 6500. Images are representative of treatment conditions from a 6-well plate, $n=4$. 
The topoisomerase II inhibitor doxorubicin also caused a morphological change to cells.

Cells were larger, but appear to have retained protrusions. This is consistent with previous results seen from our laboratory, and the flow cytometry results in figure 36. However, it is interesting to note, there appears to be no elongation to the nuclei as observed in the brightfield image in figure 30. The nuclei also appear to be larger and retain their shape. This increase in cytoplasm and nuclei size can be attributed to doxorubicin's bioactivity. As discussed in chapter 1, the DNA damage a topoisomerase II inhibitor would cause arrests the cell at the G2/M checkpoint in the cell cycle. At this checkpoint the cells are prepared to divide therefore there is an increased amount of cellular content within cell cytoplasm. Additionally, the cells have undergone $S$ phase and have twice the amount of DNA content at the G2/M checkpoint too. This accounts for the increased cytoplasm and nuclei size seen in figure 36.

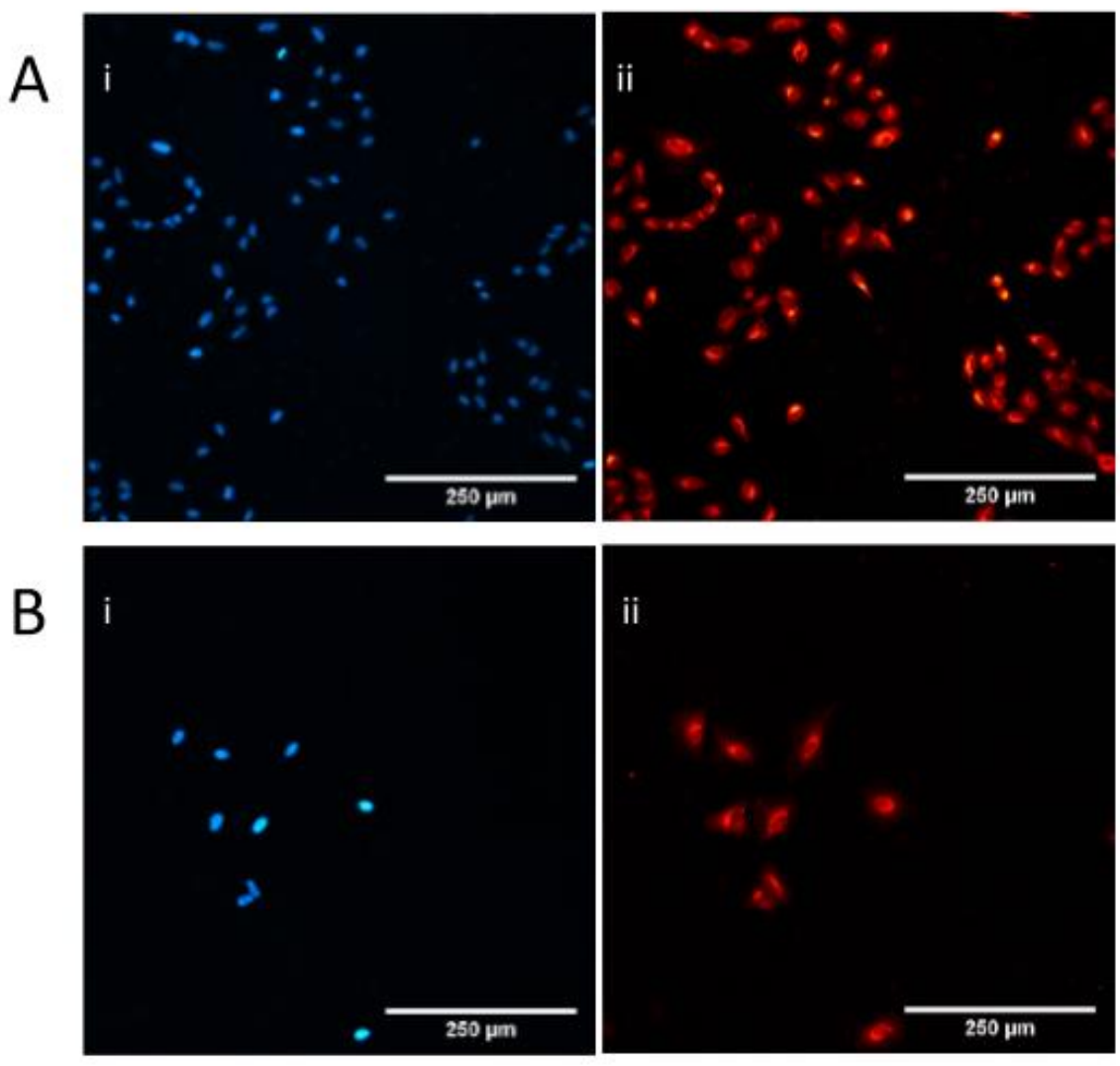

Figure 36. A549 morphology of nucleus and cell after treatment with $62 \mathrm{nM}$ of doxorubicin.

Treatments: $A=$ Control, $B=$ doxorubicin; Stains: $i=$ DAPI - nucleus stain, $i i=$ Cell Mask Deep Red Plasma Membrane. Images were taken 48 hours after treatment incubation. A549 cells were fixed and adhered to 
slides, and imaged on the IN Cell Analyser 6500. Images are representative of treatment conditions from a 6well plate, $n=4$.

BEZ235 and A66 both caused morphological changes. However, despite both being PI3K inhibitors, caused different morphological changes. BEZ235 was consistent with brightfield images (figure 37). The variability of the size and shape increased. As seen in figure 36, there is potentially some cells which have an elongated cytoplasm. The nuclei morphology did not change with BEZ235 treatment, however, there was potentially an increase in the nucleation of some cells. A66 treatment caused cells to clump (figure 36). The cytoplasm became larger, but nuclei size and shape was unchanged. 

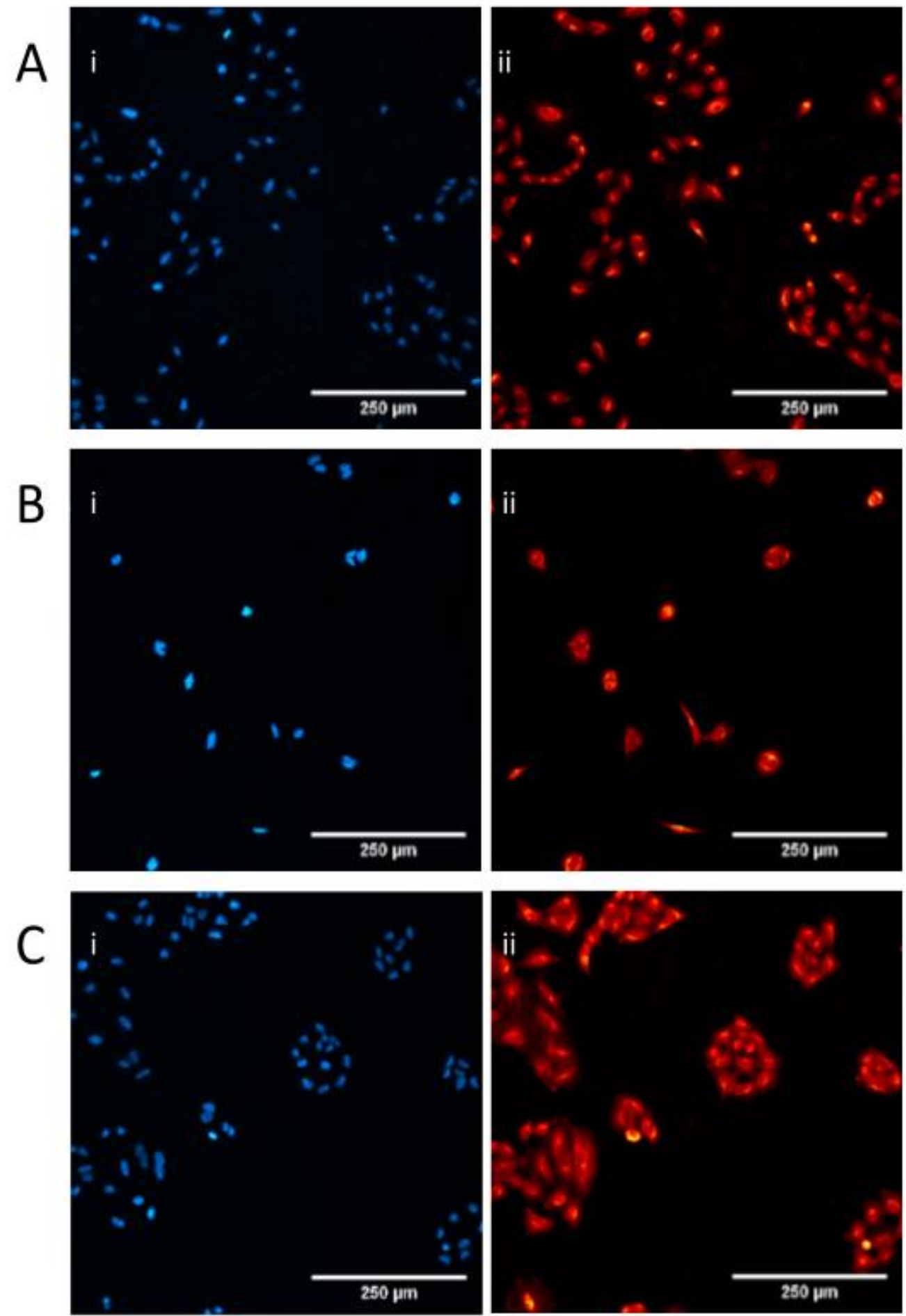

Figure 35. A549 morphology of nucleus and cell after treatment with $62 \mathrm{nM}$ of BEZ235 and $1 \mu \mathrm{M}$ of A66. Treatments: $A=$ Control, $B=$ BEZ235, $C=$ A66; Stains: $i=$ DAPI - nucleus stain, $i i=$ Cell Mask Deep Red Plasma Membrane. Images were taken 48 hours after treatment incubation. A549 cells were fixed and adhered to slides, and imaged on the IN Cell Analyser 6500. Images are representative of treatment conditions from a 6-well plate, $n=4$. 
Temozolomide, as expected, showed no morphological change compared to the control as a bioactive concentration was not used (figure 38). Once again, this exemplifies not all bioactive drugs will be used at appropriate concentration.

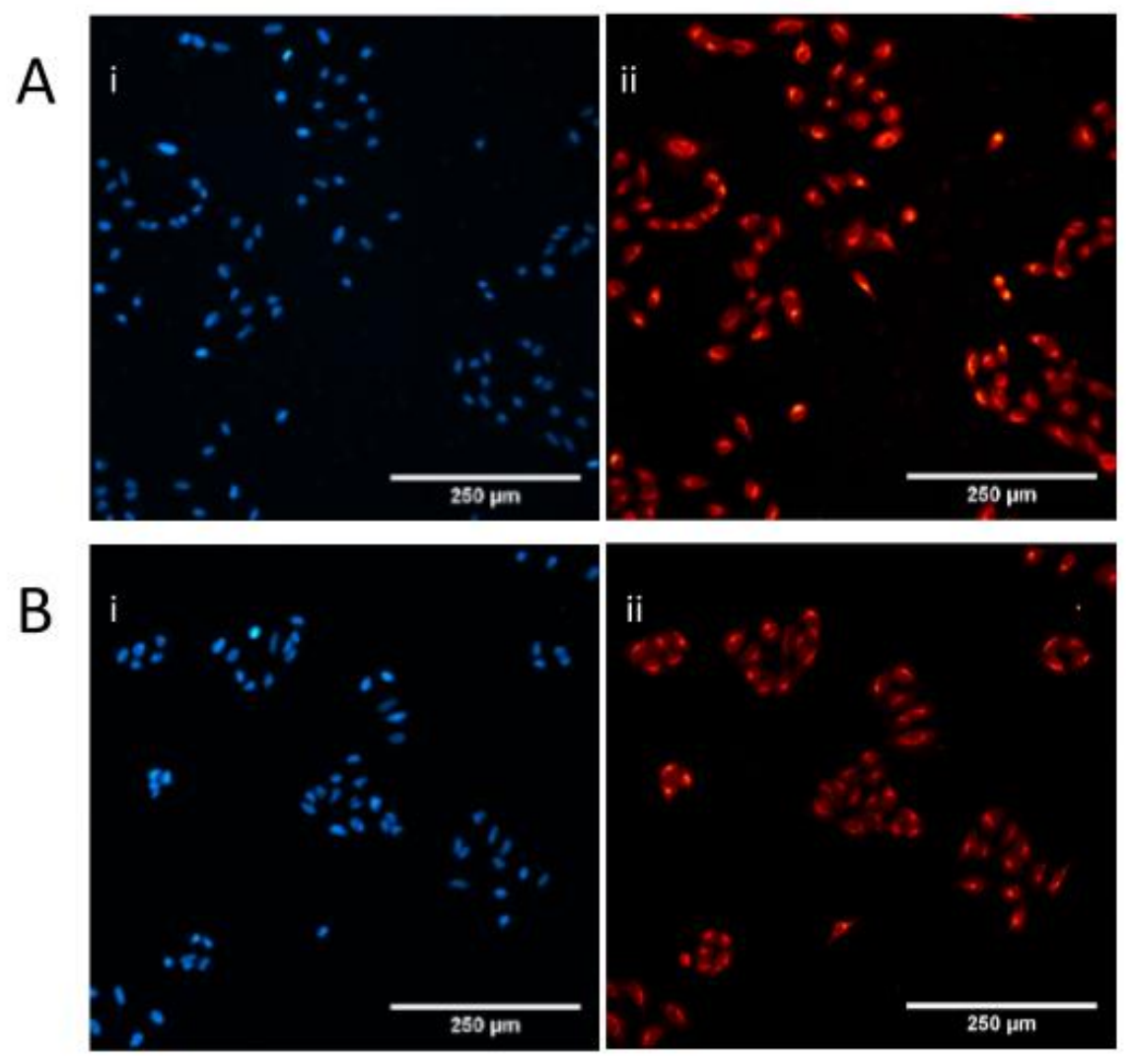

Figure 35. A549 morphology of nucleus and cell after treatment with $10 \mu \mathrm{M}$ of temozolomide.

Treatments: $A=$ Control, $B$ =temozolomide; Stains: $i=$ DAPI - nucleus stain, $i i=$ Cell Mask Deep Red Plasma Membrane. Images were taken 48 hours after treatment incubation. A549 cells were fixed and adhered to slides, and imaged on the IN Cell Analyser 6500. Images are representative of treatment conditions from a 6-well plate, $n=4$.

\subsubsection{Image analysis}

\subsubsection{CellProfiler}

The image analysis software works in a modular pipeline fashion, as shown in figure 39. Each module seen on the left hand side of the screen will process the image. The first four modules are compulsory. In these modules images are inputted, the file names are read and 
processed into groups based on what the image contains and what the sample is. The remaining modules in the pipeline are independent and alterable for the user's needs. It is here that objects, such as cells are identified and measured. Within all of the modules, parameters such as fluorescent intensity thresholds can be adjusted to assist in object identification. Once the pipeline is optimised, the images can be processed to give data output. In this case, the quantified morphological parameters of cellular components.

To quantify the morphological changes seen by eye and therefore create the MCP assay, the general method seen in figure 40 was used. As seen, CellProfiler is a crucial part to this method.

The optimised pipelines were created and assisted with the help from previous publications, ${ }^{57,60,95}$ tutorials on the CellProfiler website, ${ }^{59}$ and members of the Wellington Microscopy Group. For example, the ColorToGray module was inserted after difficulty splitting the channels from the same image file. As the module title does not allude to its function, specialist knowledge about the module would not have been discovered without assistance. The rest of the morphological quantification pipeline was created with tips described in Carpenter et al. (2006) and Lamprecht et al. (2007), as well as the help tools on CellProfiler and module explanations on the CellProfiler website. ${ }^{50,58}$ Also, the quality control CellProfiler pipeline would not have been possible without the methods described in Bray \& Carpenter (2018) and Bray et al. (2012). The first publication explicitly walks through step-by-step necessary modules and how CellProfiler Analyst would work with machine learning to categorise defective vs viable images if a database could be created. In doing so, every modules function and parameters could be understood and informed adjustments could be made if needed. 

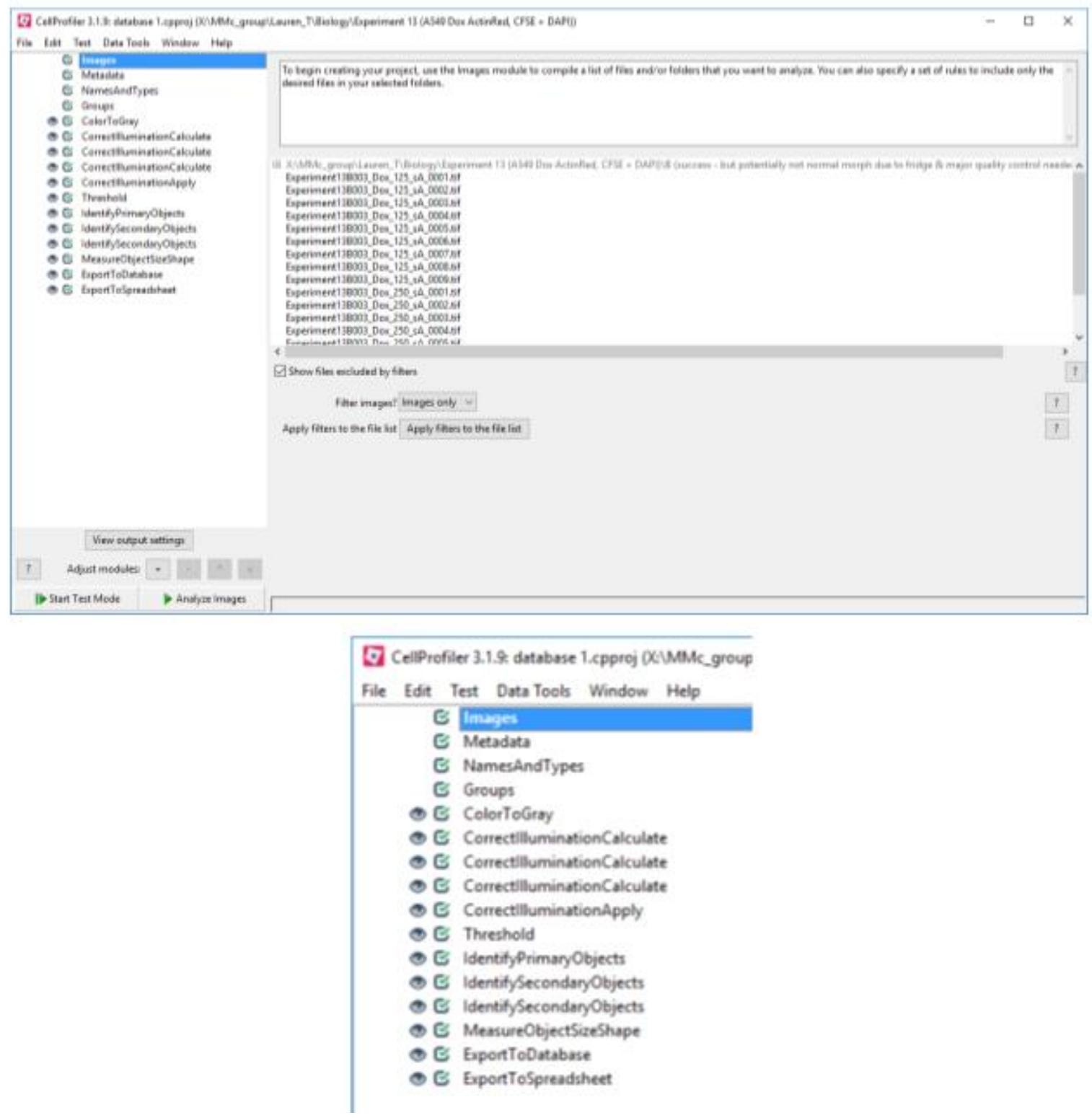

Figure 39. User Interface of CellProfiler with close up of modules.

Pipeline is displayed on left hand of screen. Modules are displayed when it is selected from the pipeline list. The "Images" module is shown here. 


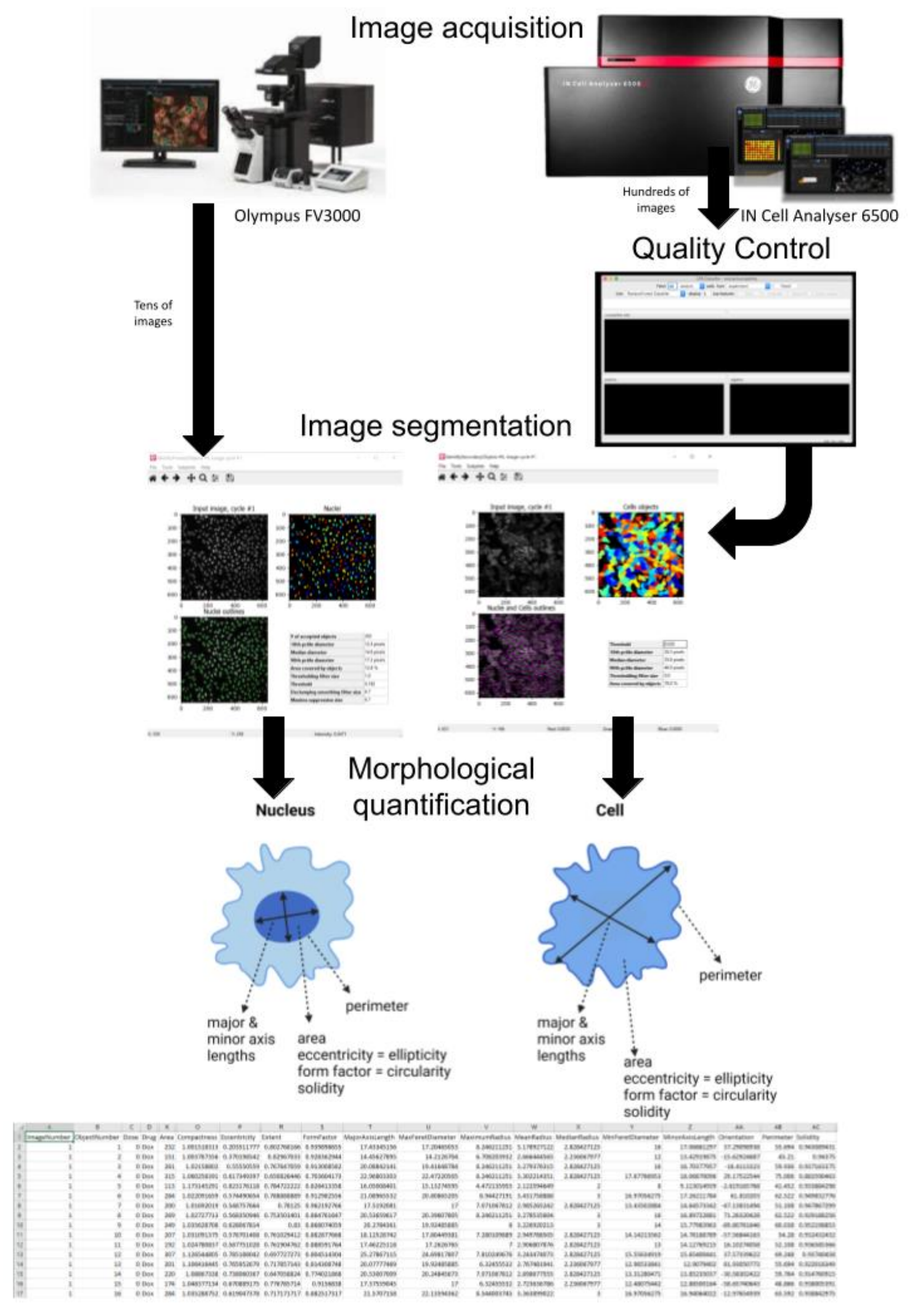

Figure 40. General method of MCP specific to Te Herenga Waka. 


\subsubsection{Preliminary pipeline optimisation}

A small number of high quality images were generated on the confocal and used to optimise the CellProfiler pipeline. The optimised pipeline can be seen in appendix 6.

An important aspect to using this software is every image fed into the pipeline must have the ability to have the fluorescent channels split by the ColorToGray module or only have one fluorescent channel shown per image. This is because in order to segment and identify specific cellular components, such as the nucleus and cytoplasm, the software measures the fluorescent intensity of the stain in the image to segment that particular cellular component it cannot distinguish between different colours within the same image.

Important modules for segmentation/object identification were IdentifyPrimaryObjects and IdentifySecondaryObjects. Their primary function was to identify and segment nuclei and cytoplasm respectively from fluorescence intensity in each image. In figure $\mathbf{4 1}$ it can be seen how these modules do this. Specifically, in the IdentifyPrimaryObjects and IdentifySecondaryObjects windows, the bottom left images show green and purple pixels. Objects circled in green pixels denote identified cell nuclei, cytoplasm and actin. The purple pixels denote objects which have been recognised in the image, but are not identified as the object of interest. The segmentation of the objects that have been recognised as nuclei and cytoplasm can be seen in the top right images of the window and denoted by the multicoloured solid blocks. When parameters within the modules changed, the objects in green and purple, as well as the solid blocks would also change. These images could be used and compared to the original image to determine if the identification and segmentation was accurate. This established confidence in the accuracy of the outputted morphological data from the MeasureObjectSizeShape and ExportToSpreadsheet modules. Particularly Important parameters within the modules were object size, thresholding bounds, and thresholding strategy and method within the Identification modules. These parameters made considerable differences to the accuracy of object identification and segmentation. Specific parameter conditions for these modules are seen in appendix 6. Increased accuracy was also seen when CorrectIlluminationCalculate, CorrectIlluminationApply and Threshold modules were added. The CorrectIllumination modules calculated and removed background illumination so the object's fluorescent intensity could be recognised more easily by the software. The Threshold module set a fluorescent intensity range in which that the object's fluorescent intensity were within so further specification to improve object identification accuracy could be made. 


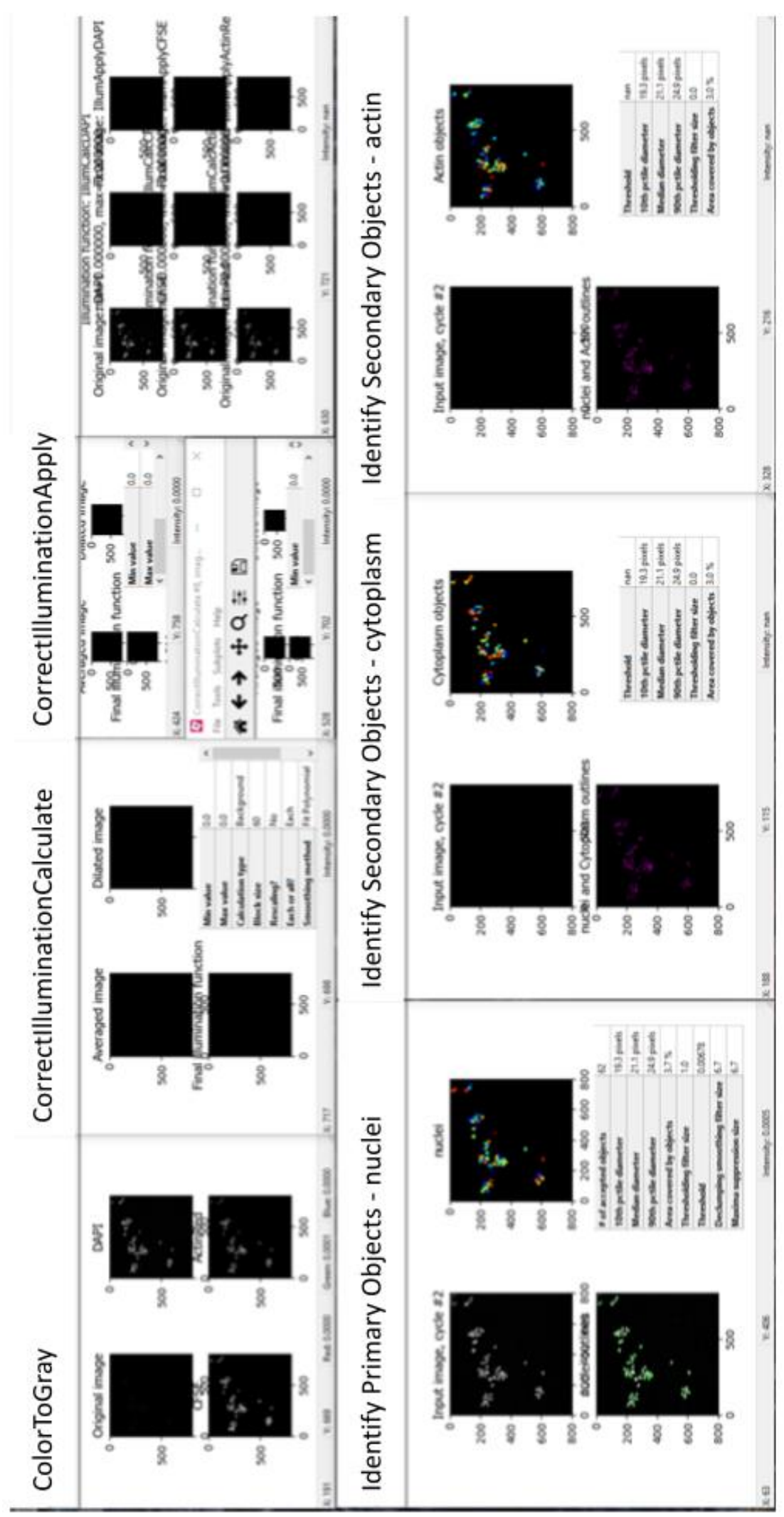

Figure 41. Data output from CellProfiler pipeline depicting image segmentation of nuclei, cytoplasm and actin. 
The pipeline's segmentation was deemed accurate through observation on the effect adjustments of the module parameters had. Twelve image sets from the Olympus FV3000 confocal were used to do this and overall, it was reasonably straight forward. Once it was deemed accurate, then approximately 70 images were analysed with the pipeline to create a spreadsheet of quantified morphological parameters of the nuclei and cytoplasm of each drug treatment's conditions. RStudio was used to create boxplots of the morphological data from the spreadsheet (figure 42). The morphological parameter measured is specified in the title of the boxplot and every dot seen constituted an object's quantified morphology. This was also reasonably straight forward and it was postulated that these boxplots could be used to create the morphobase.

With these boxplots, the robustness of the data could be determined. As seen in figure 42 the $125 \mathrm{nM}$ doxorubicin treatment (third box) had skewed results compared to the other doxorubicin treated samples. In this sample there was a reduced number of images taken from each slide and analysed. This highlighted the need for a large number of images from multiple biological replicates.
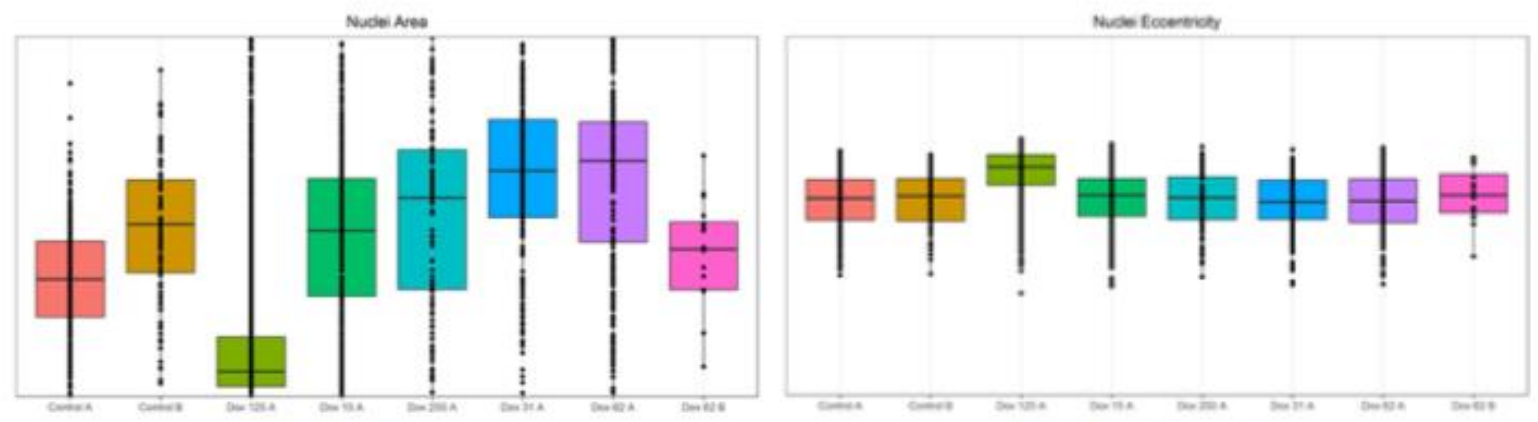

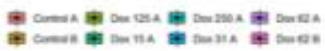

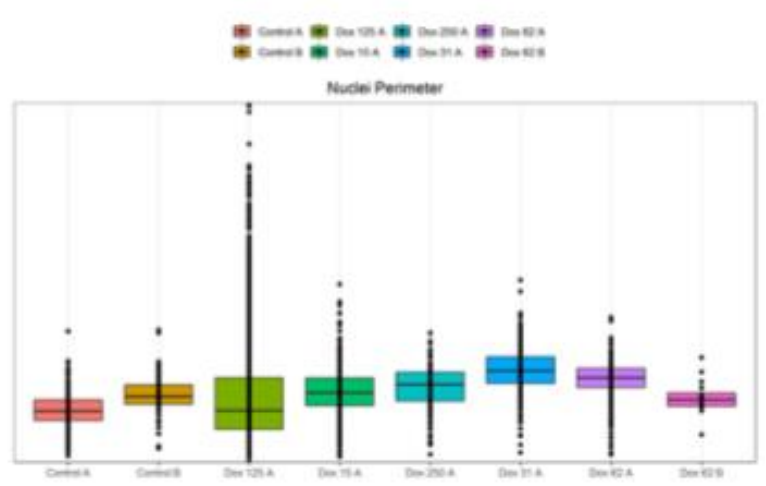

Nuces Forminactor

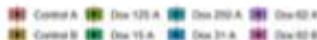

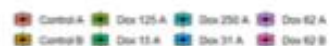

Figure 42. Examples of boxplots generated from morphological data generated by the optimised CellProfiler pipeline with images of a range of doxorubicin treated A549 cells from the Olympus FV3000. 


\subsubsection{Application of the pipeline}

Quantification of morphological features was straightforward with the few images from the Olympus FV3000. However, this data was not robust enough for a MCP assay, thus the IN Cell Analyser 6500 was used instead to increase the throughput and the number of images generated. But, from manual observation of the images, this microscope produced $\sim 30 \%$ defective images which would compromise the accuracy of the morphological parameters measured by CellProfiler, and produce unreliable data such as that shown in figure 42. To combat this, a quality control method was attempted. The adjusted general protocol taken with the IN Cell microscope can be seen in figure 40. Quality control involved the addition of a second CellProfiler pipeline to measure image quality (appendix 7) and the sister software to CellProfiler, CellProfiler Analyst (figure 43). When the pipeline is run, the database created at the end of the pipeline is then inputted into CellProfiler Analyst. This software uses a machine learning approach to separate high quality from poor quality images. Thus, would ensure the quantified morphological data generated is accurate.

At this stage, the pipeline failed to process due to the sheer quantity of images, approximately 7000 , to be analysed. The pipeline was attempted on three separate computer processors readily available to researchers. They were the humble Windows computer desktop, a Windows 10 Workstation running 2 Intel Xeon $2.2 \mathrm{GHz}$ processors with 64 GB RAM and 64 GB shared GPU memory, and Windows 2016 Server running 2 Intel Xeon $2.3 \mathrm{GHz}$ processors with 64 GB RAM. Despite this, CellProfiler crashed at every attempt. A reduction in the number of images was attempted too, and the a Windows 10 Workstation running 2 Intel Xeon $2.2 \mathrm{GHz}$ processors with 64 GB RAM and 64 GB shared GPU memory could handle processing image numbers in the tens, but not the hundreds. This ultimately led to quality control not being completed. The 7000 IN Cell images also crashed CellProfiler during an attempt to run them through the optimised morphology pipeline without quality control. This was presumably due to lack of computing power and is further discussed in the discussion. 

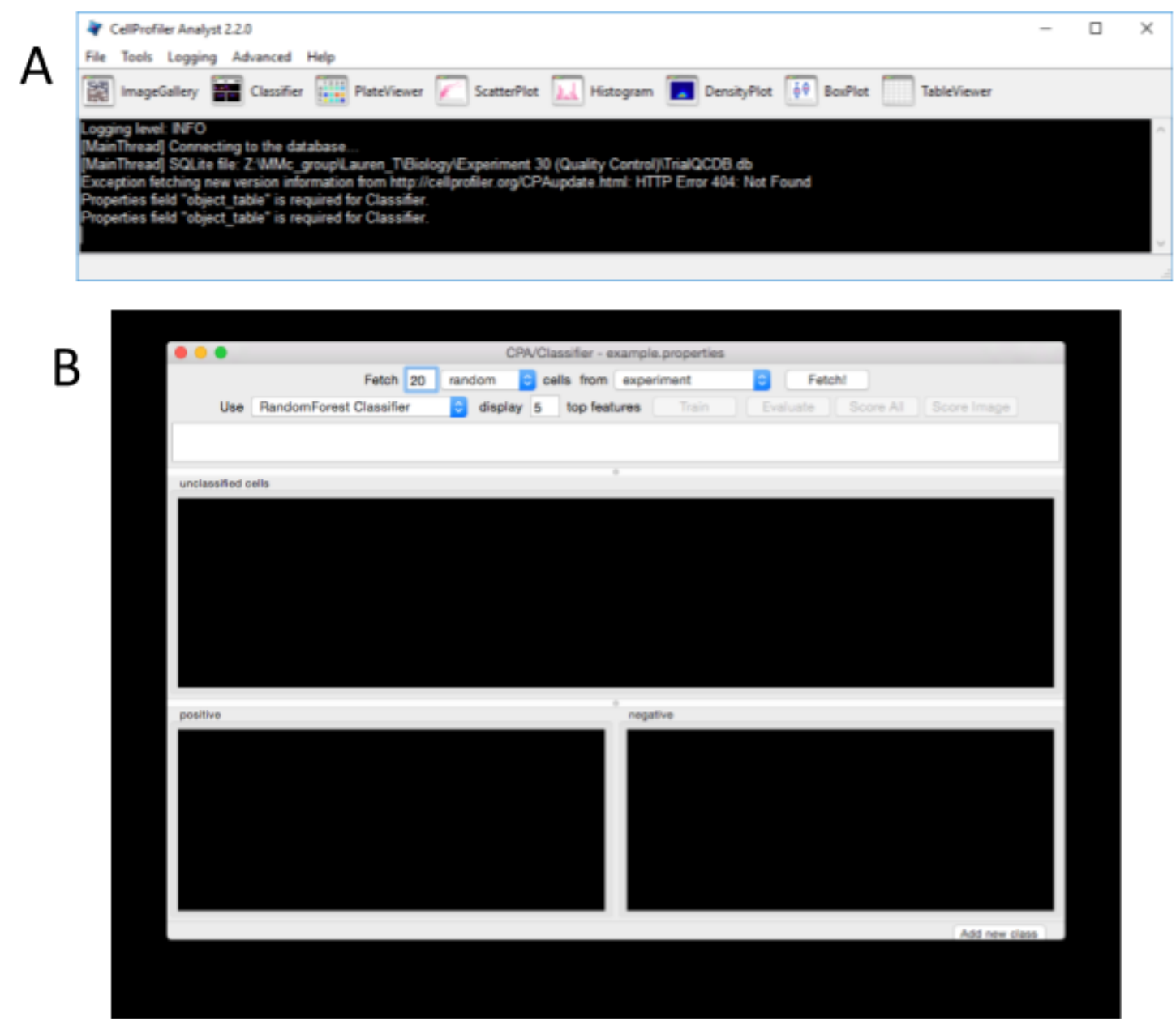

Figure 43. CellProfiler Analyst user interface.

A depicts the software home page, and B depicts the Classifier which assists with machine learning image quality. 


\subsection{Discussion}

To establish a quantitative morphological assay with CellProfiler, segmentation/object identification accuracy is crucial. As discussed in the results, segmentation was ultimately carried out by IdentifyPrimaryObjects and IdentifySecondaryObjects modules. There were two main elements that perturbed segmentation accuracy. That was cell behaviour, and image quality.

Three cell behaviours particularly perturbed accuracy, despite the A549 cell line being a well-established cell line to conduct microscopy research on. The behaviours were nucleation, too much variation in size of the nucleus, and clumping. A549 cells are shown to be mono-nucleated, have uniform morphology and be well spread apart in the control images. However, when the cells are treated with drugs, these characteristics of the cell line change. For example, BEZ235 treatment increased the number of nuclei in some cells, A66 treatment leads to condensed spacing so that the cytoplasm are overlapping more. Also, some drugs cause dramatic changes to the size of cells compared to the control. For example, doxorubicin treatment made the nuclei and cytoplasm of the cells considerably larger than the control cells.

Poly-nucleated cells are an issue to CellProfiler object identification as it will identify each nuclei from within the same cell as individual objects. Whilst this isn't an issue to the morphology results of the nucleus, it does disrupt object identification of the cytoplasm. The software identifies secondary objects, that is the cytoplasm, in a 1:1 relationship to the primary objects. Thus, if there are multiple nuclei within one cell it will try to segment one cell cytoplasm into the corresponding number of cytoplasm objects. This will disrupt the accuracy of the morphology results as the cells will be made out to be smaller than they are in reality. This issue in theory could be overcome by identifying cell cytoplasm with a IdentifyPrimaryObjects module instead so there is less reliance on mono-nucleated cells to segment the cytoplasm accurately, however, less accurate segmentation of the cytoplasm was seen when this was attempted.

Clumping or overlapping cells is an issue for segmentation as the software distinguishes one cell from another by identifying areas with low fluorescent intensity between high fluorescent intensity area. When cells clump or overlap, the fluorescent intensity doesn't reduce enough for the software to know where one cell stops and another starts. This can be improved with higher image resolution, but ultimately there are limitations even with this. 
Another concern is too much variation in size of the nucleus. As previously described segment identification relies on the nucleus being identified correctly. Part of the IdentifyPrimaryObjects module parameters that had to be specified was a rough estimate of the range of the number of pixels the objects width is. As A549 cells had uniform morphology this was not an initial concern, however when drug treatment made the cell nuclei considerably smaller or larger it was. It led to the exclusion of the nucleus and subsequently the cytoplasm from being identified if the pixel width of some nuclei were out of the specified range. Consequently, exclusion of certain cell sizes would introduce bias into the data. This pixel range was optimised, however, if the range was increased to include too low number of pixel width objects, background illumination was incorrectly identified as cell nuclei, and if this number increased to include too high number of pixel width objects, cells which were close to each other would merge and be identified as one.

Image quality perturbed the accuracy of segmentation/object identification as autofluorescent artefacts from out of focus images such as bubbles, or debris in the image could have been identified as cells. This issue however is only specific to the high-throughput IN Cell Analyser 6500 confocal as the autofocus function which makes it high-throughput isn't accurate and from manual observation, $\sim 30 \%$ of images were defective, even if a researcher has experience making slides. Understandably, this leads to inaccurate morphological data. Thus, a quality control protocol to remove these defective images had to be created to ensure accuracy of the morphological data. This is consistent with most literature which undertake a high-throughput approach to this assay. ${ }^{57,61,95}$ Protocol stated in Bray \& Carpenter (2018) and Bray et al. (2012) was used to do so, and involved creating an additional CellProfiler quality control pipeline and using CellProfiler Analyst. ${ }^{57,95}$ This in theory should increase the accuracy of the morphological data once executed.

However, the computing power to process the number of images produced by the IN Cell Analyser 6500 confocal on CellProfiler is large. Multiple efforts to adjust CellProfiler settings to process the images, but inevitably every run errored. It was concluded there was not enough computing power on these particular computers to run a pipeline due to the sheer number of images needing processing for an MCP morphobase. Multiple publications alluded to a high computing power being required, however the results from this research suggest they all massively understated this. ${ }^{57,60,61,95}$ For example, Svenningsen \& Poulsen (2019) stated "computational requirements for feature extraction are quite heavy." 61 Bray \& Carpenter (2018) also mention how only a few hundred images can be processed on a stand- 
alone desktops, but larger computing power is necessary for larger datasets. ${ }^{57}$ These were the only comments on computer processing power in these publications. One solution to this issue is Te Herenga Waka has a high performance computing cluster called Rāpoi. This was not attempted in this research, but if further experiments were conducted, the next step would be to attempt to run the CellProfiler pipelines with this computing cluster.

There were also multiple steps which required significant computational programming skills. For example, coding on RStudio. Code was written in R on RStudio by Jeffrey Sheridan, VUW to automate changing file name syntax generated by the IN Cell Analyser 6500 to one which the regular expression on CellProfiler could recognise so data could be properly organised into drug treatments and concentrations. Then to execute this code in RStudio the researcher must know how to operate RStudio, that is, set up the files necessary for the new file name data to be put into and old file name data to be extracted from; and to change parts of the code specific for the experiment. No literature cited code was necessary to assist in CellProfiler pipeline analysis. This is most likely because different microscopes were used and most likely had different automated image file syntax and the regular expression made in the Metadata module which reads the syntax was altered to fit that of the file name syntax from the microscope.

\subsection{Conclusion}

The research conducted in this chapter provides a solid foundation for an MCP to be fully established in the future at the university. The general protocol is described, the pipeline has been optimised for the microscopes at the university and code has been written to assist with the process being executed. In order to establish the assay a researcher(s) in future only needs to get the CellProfiler pipelines run on a computer cluster with large enough computer processing power, and quality control the images on CellProfiler Analyst. 
Chapter 5: Isolate compounds from

Latrunculia kaakaariki to assess if the protocol established in this project is fit for purpose 


\subsection{Introduction}

The work described in this chapter aimed to provide proof of principle that the protocol illustrated in chapters three and four is robust enough to assess the biological activity of novel natural products.

As established in the previous chapter, cell morphological changes elicited by drugs can be observed visually with fluorescence stained images, therefore bioactive compounds can be recognised without computational morphological quantification. Thus, instead of a full quantitative MCP approach, it may be more feasible and equally as informative at this current time to carry out flow cytometry and fluorescence imaging of cells at Te Herenga Waka to assess bioactivity of novel natural products.

To assess the approach, the marine sponge Latrunculia kaakaariki was investigated for natural products compounds. The genus Latrunculia has been reported to contain many bioactive compounds, for example, pyrroloiminoquinone alkaloids such as discorhabdins. ${ }^{31,39,96,97}$ In this part of the project, traditional natural product isolation and structure elucidation was carried out to supply "novel" compounds to assess the robustness of the protocols established in chapters three and four. This chapter confirms whether this project has provided an alternative protocol to assess the bioactivity of novel natural product compounds.

\subsection{Aims}

1. Isolate and elucidate the chemical structure of compounds from Latrunculia kaakaariki

2. Carry out flow cytometry and imaging of the isolates using the protocol described in chapters three and four and assess whether it is fit for purpose. 


\subsection{Results}

\subsubsection{Isolation of discorhabdin $\mathrm{E}$}

The New Zealand marine sponge Latrunculia kaakaariki was investigated for natural products. As this species is known to contain bioactive pyrroloiminoquinone alkaloids, a class of biologically active and highly coloured pigments, attentiveness towards compounds within this structural class was given throughout the isolation process. ${ }^{39-41,47,97-99}$ A depiction of the extraction and isolation method can be seen in figure 44. The sponge was extracted with methanol twice after which the extracts were passed through a reversed-phase polymeric (poly-styrene-divinyl benzene copolymer, HP20®) column. Reversed-phase columns have a non-polar stationary phase and use a polar mobile phase. Pyrroloiminoquinone alkaloids are polar compounds, therefore, their binding affinity to the non-polar stationary phase is weak. Thus, a polar mobile phase, such as a low acetone to $\mathrm{H}_{2} \mathrm{O}$ solvent mixture ratio, should elute the pyrroloiminoquinones from the column. Three fractions of decreasing polarity were collected; $30 \%, 75 \%$ and $100 \%$ acetone: $\mathrm{H}_{2} \mathrm{O}$. The $30 \%$ and $75 \%$ acetone: $\mathrm{H}_{2} \mathrm{O}$ fractions were estimated to contain pyrroloiminoquinones due to the rich colour of the fraction and the polarity of the compound structure, therefore these fractions were assessed by positive ion mass spectrometry. The most abundant ions detected in these samples were then searched in the MarinLit marine natural product database. ${ }^{100}$ MarinLit corroborated that the $30 \%$ acetone fraction contained pyrroloiminoquinone alkaloids. This $30 \%$ aqueous acetone fraction was then passed through a C18 reversed-phase semi-preparative column using HPLC. Five fractions were collected. Upon further mass spectrometry analysis, fractions A and B, and C and $\mathrm{D}$, were combined as they contained mixtures of the same ions. The combined A/B fraction's most abundant ion was detected at $\mathrm{m} / \mathrm{z} 384$. The combined C/D fraction contained two abundant ions, $\mathrm{m} / \mathrm{z} 463$ and $\mathrm{m} / \mathrm{z}$ 521. The ion $\mathrm{m} / \mathrm{z}$ 521, when compared to the MarinLit database did not correspond with any known pyrroloiminoquinone alkaloids suggesting it is possibly a new compound. However, only the combined A/B fraction was further investigated due to time restrictions and ease of purification. 


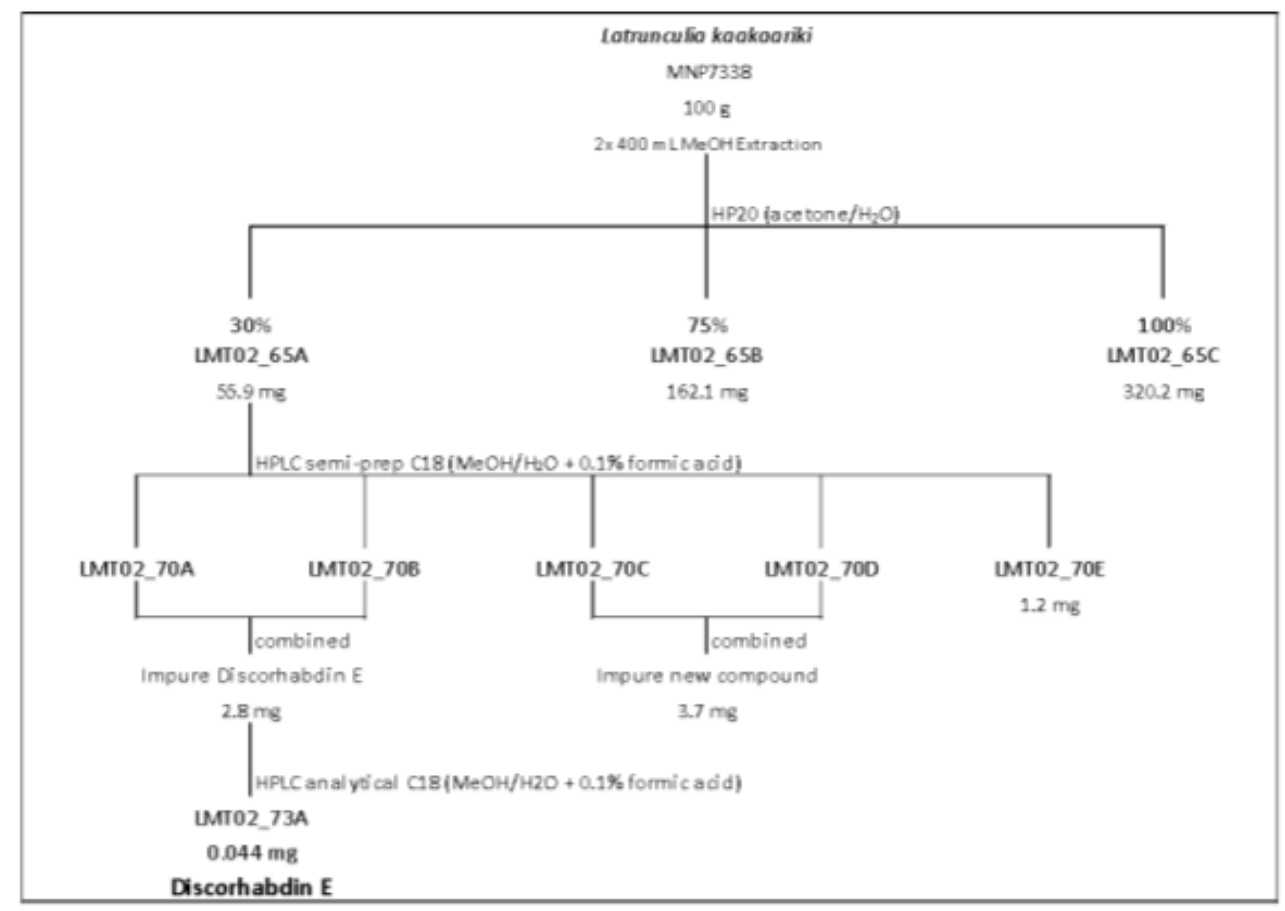

Figure 44. Extraction and isolation of Latrunculia kaakaariki.

\subsubsection{Structure elucidation of discorhabdin $\mathrm{E}$}

The combined A/B sample was a purple solid. Mass spectrometry detected a protonated ion at $m / z$ 384.037, which is consistent with the molecular formula $\mathrm{C}_{18} \mathrm{H}_{14} \mathrm{BrN}_{3} \mathrm{O}_{2}\left([\mathrm{M}+\mathrm{H}]^{+}\right.$ calculated $m / z$ 384.037) and a corresponding to an index of hydrogen deficiency (IHD) of 13. ${ }^{1} \mathrm{H}$ NMR and ${ }^{13} \mathrm{C}$ NMR spectra of the sample were obtained with $\mathrm{CD}_{3} \mathrm{OD}$ and d6-DMSO, but for the purposes of structure elucidation, the data obtained in d6-DMSO was used (table 3). This was because higher resolution was gained. The ${ }^{1} \mathrm{H}$ NMR spectrum detected 8 resonances and the ${ }^{13} \mathrm{C}$ spectrum, with the assistance of 2D-NMR HSQC and HMBC, confirmed 17 resonances. One carbon resonance remained undetected relative to the molecular formula. Of the $17{ }^{13} \mathrm{C}$ resonances, twelve were $\mathrm{sp}^{2}$ carbons, of which four were methines: $\delta_{\mathrm{C}} 178.6,158.1,157.6,157.5,154.9,124.9,124.8,122.9 ., 122.2,122.0,117.8$, 101.4. The carbon resonances at $\delta_{\mathrm{C}} 124.9(\mathrm{C}-4)$ and $157.5(\mathrm{C}-5)$ and $178.6(\mathrm{C}-3)$ with the assistance of a COSY correlation between $\delta_{\mathrm{H}} 6.22(\mathrm{~d}, \mathrm{H}-4)$ and $7.22(\mathrm{dd}, \mathrm{H}-5)$ shown in figure 45, suggested an $\alpha, \beta$-unsaturated ketone structure, accounting for two methines. The resonances of a sp ${ }^{2}$ methine $\delta_{\mathrm{C}} 157.6 / \delta_{\mathrm{H}} 7.70(\mathrm{~d}, \mathrm{H}-1)$, and quaternary $\delta_{\mathrm{c}} 122.0(\mathrm{C}-2)$ suggested a $\mathrm{C}=\mathrm{C}$ bond between one halogenated and one protonated carbon. Two HMBC 
correlations shown in figure 45, from the $\alpha$ carbon's hydrogen in the $\alpha, \beta$-unsaturated ketone to $\mathrm{C}-2$, and to quaternary $\delta_{\mathrm{C}} 44.5$ (C-6), suggested a ring system. Together this entire ring system accounted for four degrees of saturation.

Two methylene bridges were observed. One was accounted for by $\delta_{\mathrm{c}} 34.0 / \delta_{\mathrm{H}} 1.82\left(\mathrm{~m}, \mathrm{CH}_{2}-\right.$ 7) and $\delta_{\mathrm{C}} 37.5 / \delta_{\mathrm{H}}, 3.42\left(\mathrm{t}, \mathrm{CH}_{2}-8\right)$, linked by a mutual strong COSY correlation (figure 46). The other was accounted for by $\delta_{\mathrm{C}} 49.9 / \delta_{\mathrm{H}} 3.86\left(\mathrm{dd}, \mathrm{CH}_{2}-17\right)$ and $\delta_{\mathrm{C}} 18.4 / \delta_{\mathrm{H}} 2.54\left(\mathrm{dd}, \mathrm{CH}_{2}-\right.$ 16), with a corresponding COSY correlation (figure 45).

Shifts $\delta_{\mathrm{C}} 124.8, \delta_{\mathrm{H}} 7.05$ (s, CH-14); $\delta_{\mathrm{c}} 117.8(\mathrm{C}-15), 122.9(\mathrm{C}-21)$, and $\delta_{\mathrm{c}} 122.2(\mathrm{C}-12)$ implied a pyrrole structure, and therefore accounted for the fourth and final methine. HMBC correlations from $\delta_{\mathrm{H}} 7.05$ to $\delta_{\mathrm{C}} 122.9$ and $\delta_{\mathrm{C}} 117.9$ confirmed this (figure 45). There was also a carbon resonance at $\delta_{\mathrm{C}} 158.1$, which suggested a double bond to an electronegative heteroatom, consistent with an imine function. $\mathrm{HMBC}$ correlations from $\delta_{\mathrm{H}} 3.86\left(\mathrm{H}_{2}-17\right)$ to $\delta_{\mathrm{C}} 158.1\left(\mathrm{C}-19\right.$ and to $\delta_{\mathrm{C}} 117.8(\mathrm{C}-15)$ were also detected that linked the pyrrole to the imine (figure 45). Overall, five degrees of saturation are accounted for by these shifts and correlations.

Together this spectral analysis suggested the presence of a pyrroloiminoquinone alkaloid structure. When both the NMR and MS data was compared to MarinLit, two constitutional pyrroloiminoquinone isomers were possible, discorhabdin E and discorhabdin G. The remaining degrees of saturation are satisfied by these two compounds. From this structure elucidation and the presence of an $\alpha, \beta$-unsaturated ketone structure, it was deduced that combined fraction A/ B was discorhabdin E. 
Table 3. NMR data of discorhabdin E (d $\left.6^{-D M S O}\right)$.

\begin{tabular}{|c|c|c|c|c|c|c|c|c|}
\hline \multirow[b]{2}{*}{ Position } & \multirow{2}{*}{$\frac{{ }^{13} \mathrm{C}}{\delta(\mathrm{ppm})}$} & \multirow[b]{2}{*}{ HSQC } & \multicolumn{3}{|c|}{${ }^{1} \mathrm{H}$} & \multirow[b]{2}{*}{ cosy } & \multirow[b]{2}{*}{ HMBC } & \multirow[b]{2}{*}{ function $\left({ }^{13} \mathrm{C}\right)$} \\
\hline & & & $\delta(\mathrm{ppm})$ & mult & $\mathrm{J}(\mathrm{Hz})$ & & & \\
\hline 1 & 157.6 & $\mathrm{CH}$ & 7.70 & $d$ & 2.7 & 5 & - & $\mathrm{C}=\mathrm{C}$ \\
\hline 2 & 122.0 & - & - & - & - & - & 1,4 & $\mathrm{C}=\mathrm{C}, \mathrm{C}-\mathrm{Br}$ \\
\hline 3 & 178.6 & - & - & - & - & - & 1 & $C=O$ \\
\hline 4 & 124.9 & $\mathrm{CH}$ & 6.22 & $d$ & 9.7 & 5 & - & $C=C$ \\
\hline 5 & 157.5 & $\mathrm{CH}$ & 7.22 & dd & $10.0,2.7$ & 4,1 & 7 & $C=C$ \\
\hline 6 & 44.5 & - & - & - & - & - & 4,7 & C-C \\
\hline 7 & 34.0 & $\mathrm{CH} 2$ & 1.82 & $\mathrm{~m}$ & - & 8 & - & $\mathrm{CH} 2$ \\
\hline 8 & 37.5 & $\mathrm{CH} 2$ & 3.42 & $\mathrm{~m}$ & - & 7 & - & $\mathrm{CH} 2, \mathrm{C}-\mathrm{N}$ \\
\hline 9 & - & - & - & - & - & - & - & - \\
\hline 10 & 154.9 & - & - & - & - & - & 8 & $C=C$ \\
\hline 11 & - & - & - & - & - & - & - & - \\
\hline 12 & 122.2 & - & - & - & - & - & - & $C=C$ \\
\hline 13 & - & - & - & - & - & - & - & - \\
\hline 14 & 124.8 & $\mathrm{CH}$ & 7.05 & $\mathrm{~s}$ & - & - & - & $C=C$ \\
\hline 15 & 117.8 & - & - & - & - & - & 14,17 & $C=C$ \\
\hline 16 & 18.4 & $\mathrm{CH} 2$ & 2.54 & $\mathrm{dt}$ & $9.8,3.2$ & 17 & 17 & $\mathrm{CH} 2$ \\
\hline 17 & 49.9 & $\mathrm{CH} 2$ & 3.86 & dd & $14.9,8.8$ & 16 & - & $\mathrm{C}-\mathrm{N}, \mathrm{C}-\mathrm{H}$ \\
\hline 18 & - & - & - & - & - & - & - & - \\
\hline 19 & 158.1 & - & - & - & - & - & 1 & $\mathrm{C}=\mathrm{N} ?$ \\
\hline 20 & 101.4 & - & - & - & - & - & 7 & $\mathrm{C}=\mathrm{C}$ \\
\hline 21 & 122.9 & - & - & - & - & - & 14 & $\mathrm{C}=\mathrm{C}$ \\
\hline
\end{tabular}



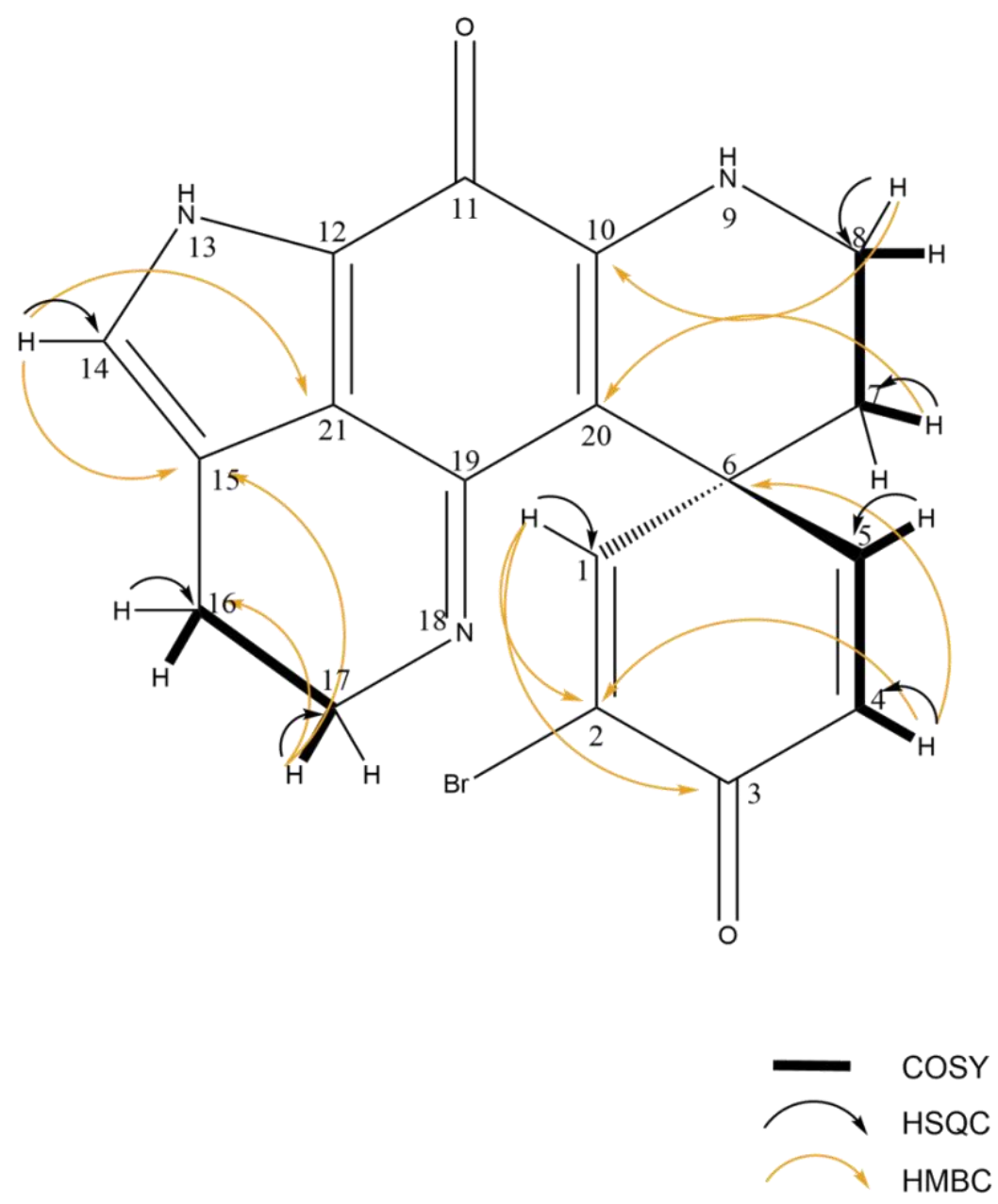

Figure 45. Schematic of COSY, HSQC and HMBC correlations superimposed onto discorhabdin E.

\subsubsection{Discussion to confirm discorhabdin $\mathrm{E}$, not discorhabdin $\mathrm{G}$}

To confirm the sample was discorhabdin E more investigations to compare the structures of discorhabdin $\mathrm{E}$ and $\mathrm{G}$ to the NMR dataset obtained and previous literature were made.

The molecular formulae of discorhabdins $\mathrm{E}$ and $\mathrm{G}$ are identical, hence the same potential ion $\mathrm{m} / \mathrm{z} 384.037$ would be observed using MS for either compound. The two compounds only differ by the placement of an alkene function (figure 46); in discorhabdin E, the double bond is placed between carbons 4 and 5, whilst in discorhabdin $\mathrm{G}$ the double bond is between carbons 7 and 8 . In theory, and as supported in the structure elucidation above, this structural difference is observable by NMR. 


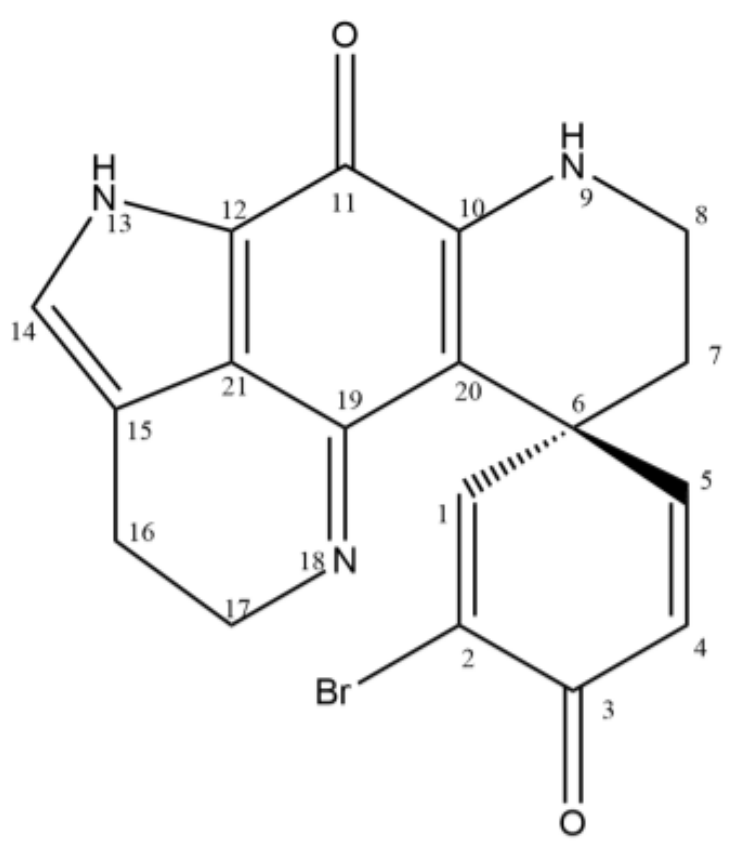

Discorhabdin E

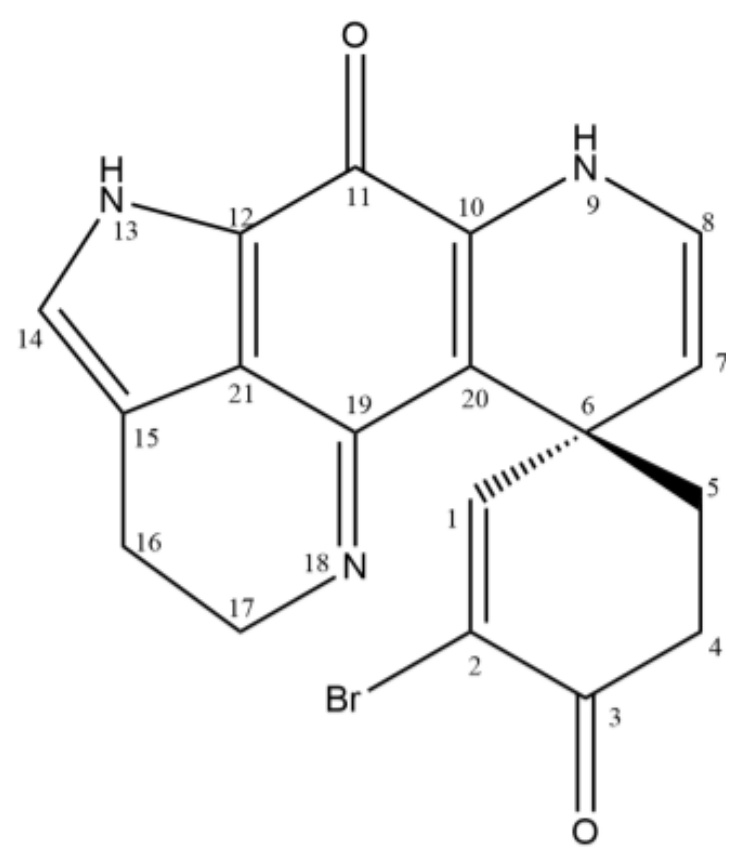

Discorhabdin G

Figure 46. Chemical structures of discorhabdin E and G.

Modified versions of the ${ }^{1} \mathrm{H}$ NMR and ${ }^{13} \mathrm{C}$ NMR characterisation tables of discorhabdin $\mathrm{E}$ and $\mathrm{G}$ from literature are seen in tables $4 \& 5$. In the literature ${ }^{13} \mathrm{C} N \mathrm{NM}$ data, there are expected notable differences in chemical shifts for carbon's 3, 4, 5, 7, 8 and 10 between discorhabdin $\mathrm{E}$ and $\mathrm{G}$. The most recognisable difference is the shifts of the carbon resonances of the distinctive double bond; that is carbons 4 and 5 in E, and 7 and 8 in $\mathrm{G}$. The placement of this double bond deshields the ${ }^{13} \mathrm{C}$ NMR chemical shift to a higher frequency. However, this alone cannot distinguish which discorhabdin the sample is as both species observe this effect with their respective double bonds. Further analysis of the shift disparities due to the effect neighbouring atoms would have is necessary. Discorhabdin E contains a doubly $\alpha, \beta-$ unsaturated ketone, including the $\Delta 4,5$ double bond, whereas discorhabdin G's alkene function is placed beside a secondary amine function. As discussed above, the shifts of C-4 ( $\left.\delta_{\mathrm{C}} 124.9\right)$, C-5 $(\delta \mathrm{c} 157.5)$ and C-3 ( $\left.\delta_{\mathrm{C}} 178.6\right)$, with the assistance of the COSY correlation between H-4 and $\mathrm{H}-5$ suggested an $\alpha, \beta$-unsaturated ketone structure, confirming discorhabdin $\mathrm{E}$. This is because a neighbouring carbonyl elicits more deshielding of the $\beta$ carbon in an alkene bond 
than a secondary amine would, due to conjugation effects. Accordingly, C-5 in discorhabdin E has a more deshielded chemical shift, at approximately $154 \mathrm{ppm}$. This is higher than any alkene carbon chemical shift in discorhabdin $\mathrm{G}$, the highest being the C-8 chemical shift at approximately $124 \mathrm{ppm}$. The NMR data for the combined fraction A/B sample was obtained in $d_{6}$-DMSO. Previous literature NMR data were obtained in $\mathrm{CD}_{3} \mathrm{OD}$, thus it was expected the shifts for the sample would be slightly different to literature values, albeit the chemical shifts would still be comparable as the compound was subject to the same deshielding effects.

Once the compound was fully purified (except minor contamination with formic acid), a second ${ }^{1} \mathrm{H}$ NMR spectrum was collected in $\mathrm{CD}_{3} \mathrm{OD}$. It not only affirmed purity of the sample, but also provided NMR data that was consistent with discorhabdin E's characterisation in the literature. A paucity of isolated pure discorhabdin E $(0.044 \mathrm{mg})$ prevented acquiring any ${ }^{13} \mathrm{C}$ NMR data for additional comparison. 
Table 4. Experimental and literature ${ }^{1} \mathrm{H}(600 \mathrm{MHz})$ and ${ }^{13} \mathrm{C} N \mathrm{NR}$ data of discorhabdin $\mathrm{E}$ and $\mathrm{G}$ in $\mathrm{CD}_{3} \mathrm{OD}$, and compared to Fraction $70 \mathrm{~A} / \mathrm{B}$.

Tables were adapted from Copp et al. (1994) and Yang \& Baker (1995).

\begin{tabular}{|c|c|c|c|c|c|c|c|c|c|c|c|}
\hline \multicolumn{4}{|c|}{ Discorhabdin E literature NMR in MeOD } & \multicolumn{4}{|c|}{ Discorhabdin $\mathrm{G}$ literature NMR in MeOD } & \multicolumn{4}{|c|}{ Fraction $70 \mathrm{~A} / \mathrm{B}$ NMR in $\mathrm{d}_{6}$-DMSO } \\
\hline \multirow[b]{2}{*}{ Position } & \multirow{2}{*}{$\begin{array}{c}{ }^{13} \mathrm{C} \\
\delta(\mathrm{ppm})\end{array}$} & \multicolumn{2}{|c|}{${ }^{1} \mathrm{H}$} & \multirow[b]{2}{*}{ Position } & \multirow{2}{*}{$\begin{array}{c}{ }^{13} \mathrm{C} \\
\delta(\mathrm{ppm})\end{array}$} & \multicolumn{2}{|c|}{${ }^{1} \mathrm{H}$} & \multirow[b]{2}{*}{ Position } & \multirow{2}{*}{$\begin{array}{c}{ }^{13} \mathrm{C} \\
\delta(\mathrm{ppm})\end{array}$} & \multicolumn{2}{|c|}{${ }^{1} \mathrm{H}$} \\
\hline & & $\delta(\mathrm{ppm})$ & mult & & & $\delta(\mathrm{ppm})$ & mult & & & $\delta(\mathrm{ppm})$ & mult \\
\hline 1 & 152.8 & 7.70 & d & 1 & 153.1 & 7.64 & s & 1 & 157.6 & 7.70 & d \\
\hline 2 & 127.0 & - & - & 2 & 125.3 & - & - & 2 & 122.0 & - & - \\
\hline 3 & 179.0 & - & - & 3 & 190.3 & - & - & 3 & 178.6 & - & - \\
\hline 4 & 129.9 & 6.55 & d & 4 & 32.8 & $2.5,3.0$ & ddd, $m$ & 4 & 124.9 & 6.22 & d \\
\hline 5 & 153.2 & 7.19 & dd & 5 & 36.4 & $\begin{array}{l}2.31 \\
2.68\end{array}$ & $\mathrm{~m}, \mathrm{br} d \mathrm{dt}$ & 5 & 157.5 & 7.22 & dd \\
\hline 6 & 44.2 & - & - & 6 & 43.0 & - & - & 6 & 44.5 & - & - \\
\hline 7 & 35.7 & 2.05 & $\mathrm{~m}$ & 7 & 113.2 & 5.40 & d & 7 & 34.0 & 1.82 & $\mathrm{~m}$ \\
\hline 8 & 39.9 & 3.70 & $t$ & 8 & 124.7 & 6.38 & d & 8 & 37.5 & 3.42 & $\mathrm{~m}$ \\
\hline 9 & - & - & - & 9 & - & - & - & 9 & - & - & - \\
\hline 10 & 154.1 & - & - & 10 & 146.7 & - & - & 10 & 154.9 & - & - \\
\hline 11 & 166.9 & - & - & 11 & 167.5 & - & - & 11 & - & - & - \\
\hline 12 & 125.1 & - & - & 12 & 122.9 & - & - & 12 & 122.2 & - & - \\
\hline 13 & - & - & - & 13 & - & - & - & 13 & - & - & - \\
\hline 14 & 128.0 & 7.18 & $\mathrm{~s}$ & 14 & 127.4 & 7.16 & $\mathrm{~s}$ & 14 & 124.8 & 7,05 & $\mathrm{~s}$ \\
\hline 15 & 121.6 & - & - & 15 & 121.0 & - & - & 15 & 117.8 & - & - \\
\hline 16 & 19.8 & 2.87 & $t$ & 16 & 19.3 & 2.94 & $t$ & 16 & 18.4 & 2.54 & dt \\
\hline 17 & 45.5 & 3.77 & $\mathrm{t}$ & 17 & 45.9 & 3.89 & $\mathrm{t}$ & 17 & 49.9 & 3.86 & dd \\
\hline 18 & - & - & - & 18 & - & - & - & 18 & - & - & - \\
\hline 19 & 156.6 & - & - & 19 & 160.0 & - & - & 19 & 158.1 & - & - \\
\hline 20 & 94.0 & - & - & 20 & 100.7 & - & - & 20 & 101.4 & - & - \\
\hline 21 & 125.5 & - & - & 21 & 123.7 & - & - & 21 & 122.9 & - & - \\
\hline
\end{tabular}


So precise concentrations could be made for biological experiments, quantification of the discorhabdin E sample using qNMR was completed. There appeared to be residual formic acid in the sample from the HPLC purification so formic acid was also quantified such that it could be controlled for in subsequent biological experiments.

\subsubsection{Bioactivity assessment of discorhabdin E by flow cytometry}

Despite discorhabdin E being a known compound, the following experiments were attempted as if it were a new, biologically uncharacterised compound. The rules to find the Goldilocks concentration as stated in chapter three were followed. Cytotoxicity assays were completed at a range of concentrations of discorhabdin E between $125 \mathrm{nM}$ to $1 \mu \mathrm{M}$ first (figure 47). The results showed no change in the percentage of affected cells or cell death as concentration increased. A slight increase of affected cells and cell death can be seen at $1 \mu \mathrm{M}$, but whether this is a notable change is not evident. Due to this, the compound was retested at $10 \mu \mathrm{M}$, as per the rules (figure 47). The compound was toxic at this concentration with almost all cells affected, and $\sim 70 \%$ of them dead.

The formic acid contamination was controlled for at $1 \mu \mathrm{M}$ and $10 \mu \mathrm{M}$ discorhabdin $\mathrm{E}$ equivalent amounts. That is, the amount of formic acid that would have been transferred across from the sample at the specified concentration of discorhabdin E was tested separately. The formic acid alone showed no increased level of toxicity compared to the controls (figure 47). This suggested the amount of formic acid that was present in the discorhabdin E sample did not have an effect on the cells, and that discorhabdin E alone was toxic. 


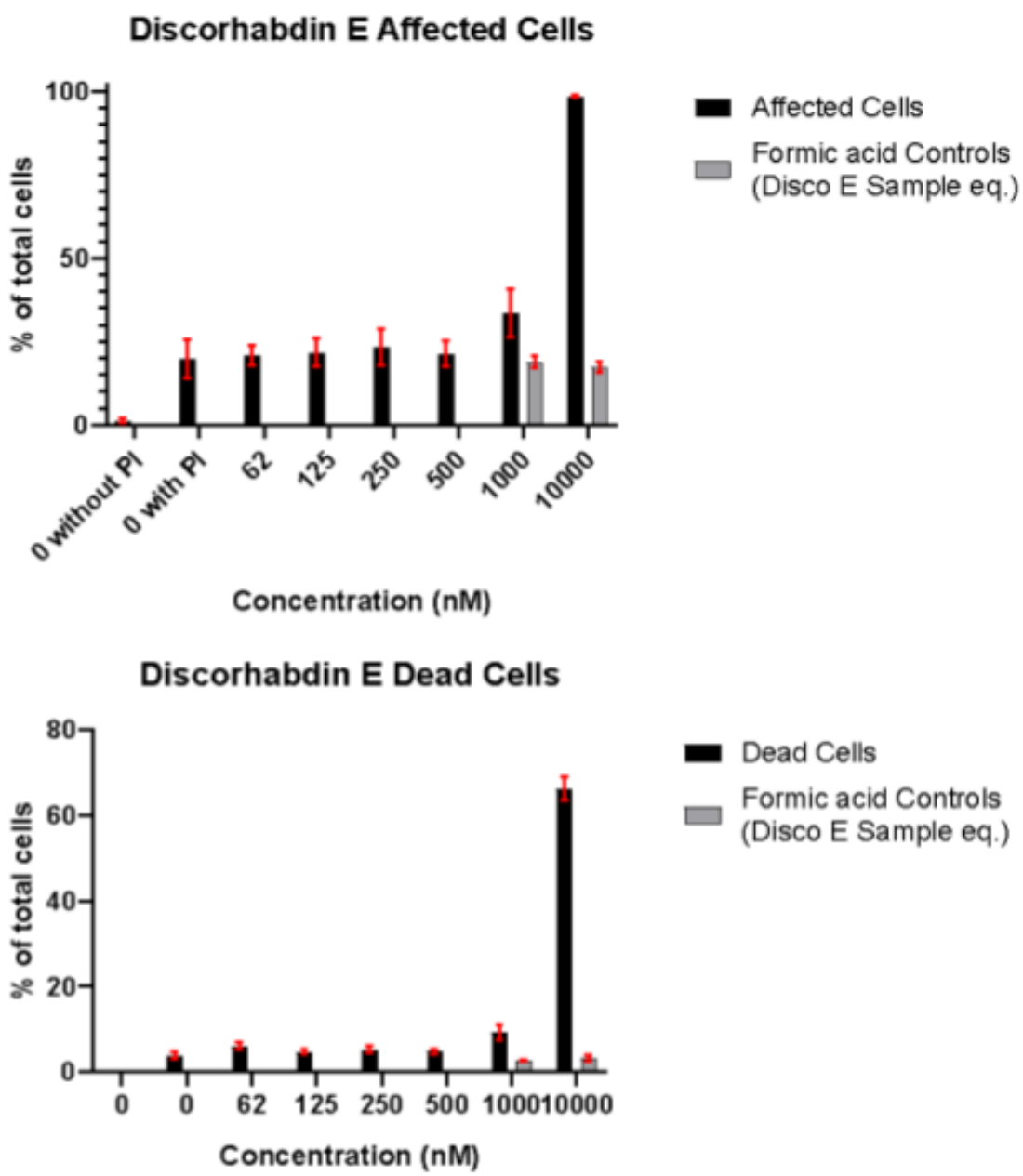

Figure 47. Discorhabdin E treatment affected and killed A549 cells.

Results were acquired by flow cytometry. Error bars $=$ SEM; Controls with and without PI were made to assist with gating. Formic acid controls were added to control for the residual amounts present within the discorhabdin treatments.

The flow cytometry cell morphology parameters were also analysed, and results are shown in figure 48. This was done as per the protocol in chapter three to supplement the cytotoxicity results (figure 47). The lower concentrations of discorhabdin E were tested, $125 \mathrm{nM}$ to 500 $\mathrm{nM}$ were the same as untreated cells - no change in morphology was observed. However, at 1 $\mu \mathrm{M}$, there was a morphological change. Cell size became biphasic. A proportion of cells became larger, whilst a smaller proportion became smaller. This smaller cell size population is consistent with a higher proportion of dead cells, as seen with the previous exemplary drugs. Cell granularity increased. In the $10 \mu \mathrm{M}$ treatment results, most cells decreased in size. 
Again this is consistent with a higher proportion of cell death, thus consistent with cytotoxicity results showing a large proportion of cell death occurred. Cell granularity had also increased at this concentration. In all samples no cellular debris was observed which shows at all concentrations no cells were completely obliterated. Together these results suggest at $1 \mu \mathrm{M}$ discorhabdin $\mathrm{E}$ is bioactive, and at $10 \mu \mathrm{M}$ it is cytotoxic.

The formic acid control samples showed no change in cell size or granularity, reinforcing that formic acid was not bioactive at the concentrations of formic acid that would have been present in the $1 \mu \mathrm{M}$ and $10 \mu \mathrm{M}$ discorhabdin E samples.
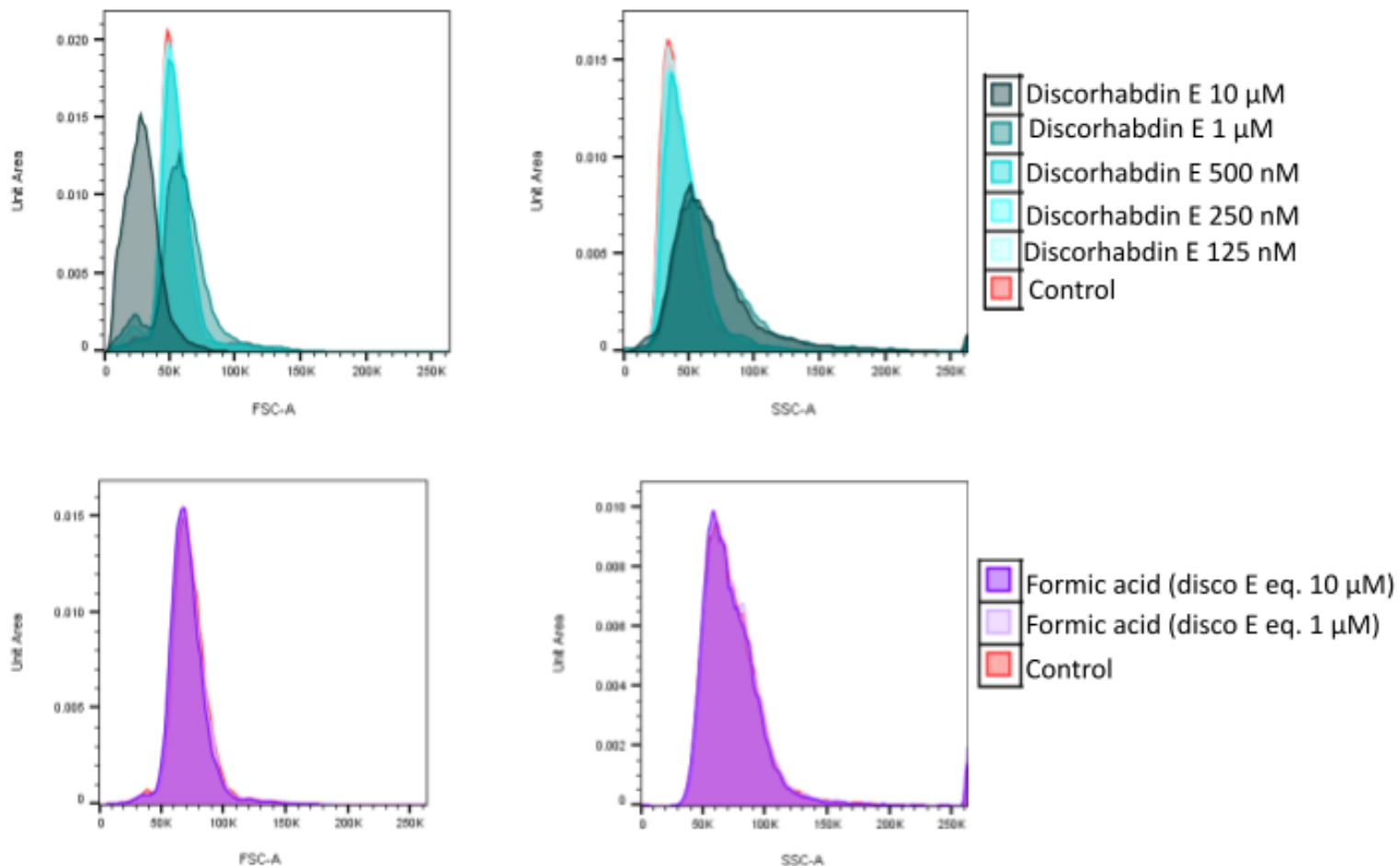

Figure 48. Discorhabdin E treatment affected forward scatter (FSC) and cell granularity (SSC).

Results were acquired by flow cytometry synonymous with cytotoxicity data. Legend labels with treatment concentration are on the right. Control is shown in red and the tone of blue and purple for the treatment samples of discorhabdins and formic acid gets darker as concentration increases respectively. 
As discorhabdin $\mathrm{E}$ was shown to be bioactive and not notably cytotoxic at $1 \mu \mathrm{M}$ in the flow cytometry morphology results, images of cells at discorhabdin $\mathrm{E}$ treatment concentrations of $1 \mu \mathrm{M}$ and $2 \mu \mathrm{M}$ were taken (figure 49). Formic acid discorhabdin E equivalents at these concentrations were also made (figure 50). In both $1 \mu \mathrm{M}$ and $2 \mu \mathrm{M}$ discorhabdin $\mathrm{E}$ treatments, a biphasic cell size can be seen, a small proportion of them are small and large proportion of them are similar in size to the control cells. This is consistent with flow cytometry morphology results. It also suggests there is a population of small cells which had not died. This is because dead cells do not usually remain adhered to the cover slip for imaging. There was also a larger proportion of small cells in the $2 \mu \mathrm{M}$ treatment images. This suggests the proportion of the small cell size population increases in a concentration dependent manner. Additionally, nuclei size can be seen in these images. Notably, the cells which have a small size also have a small nucleus.

As expected, there was no change in cell morphology for the formic acid controls. Once again, this confirms formic acid had no considerable effect at concentrations equivalent that would have been present in the discorhabdin E samples. 

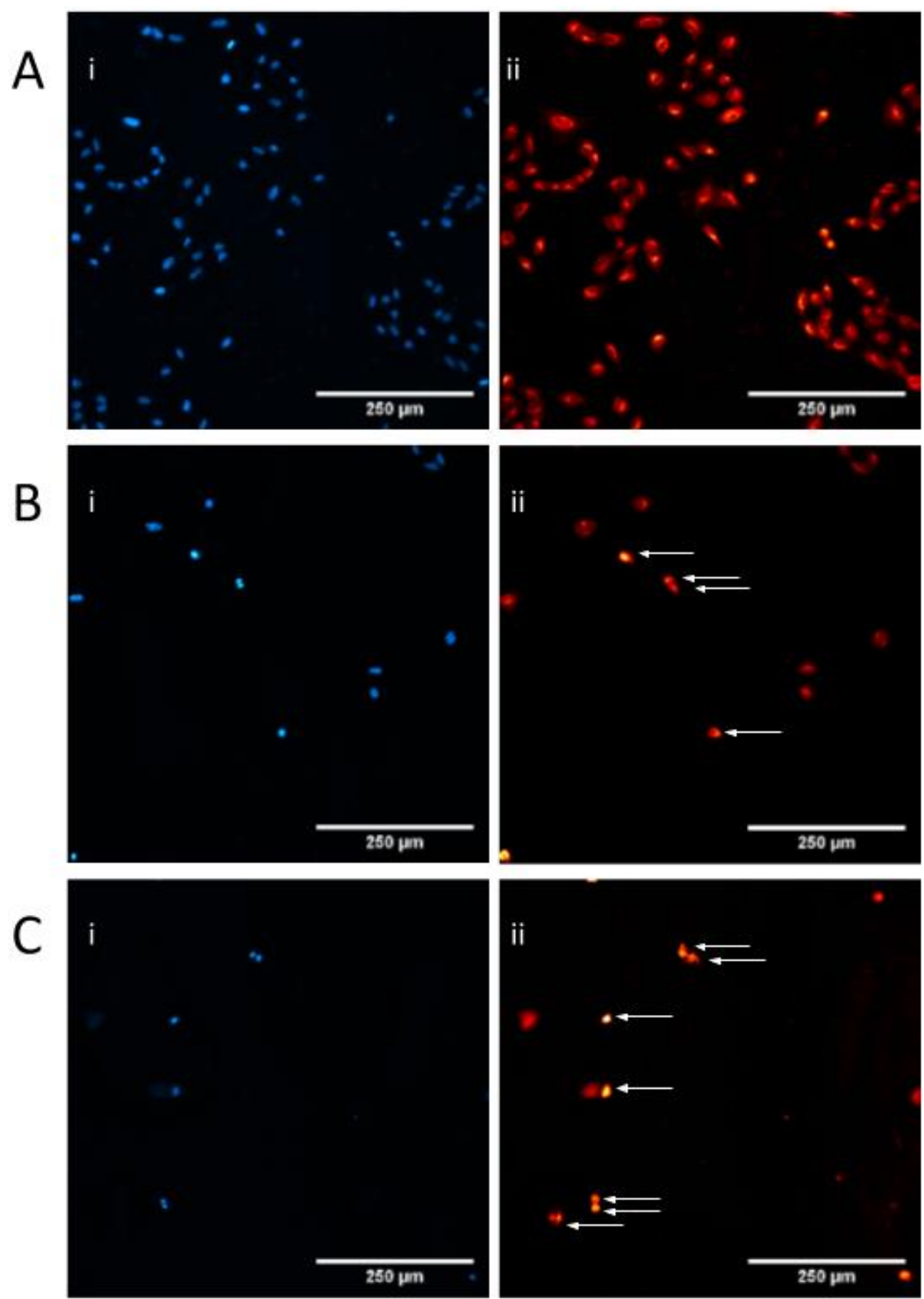

Figure 49. A549 morphology of nucleus and cell after treatment with $1 \mu M$ and $2 \mu M$ of Discorhabdin $E$.

Treatments: $A=$ Control, $B=$ Discorhabdin $E(1 \mu M), C=$ Discorhabdin $E(2 \mu M)$; Stains: $i=D A P I-$ nucleus stain, ii = Cell Mask Deep Red Plasma Membrane. Small cells are labelled with an arrow. Images were taken 48 hours after treatment incubation. A549 cells were fixed and adhered to slides, and imaged on the IN Cell Analyser 6500. Images are representative of treatment conditions from a 6 -well plate, $n=4$. 


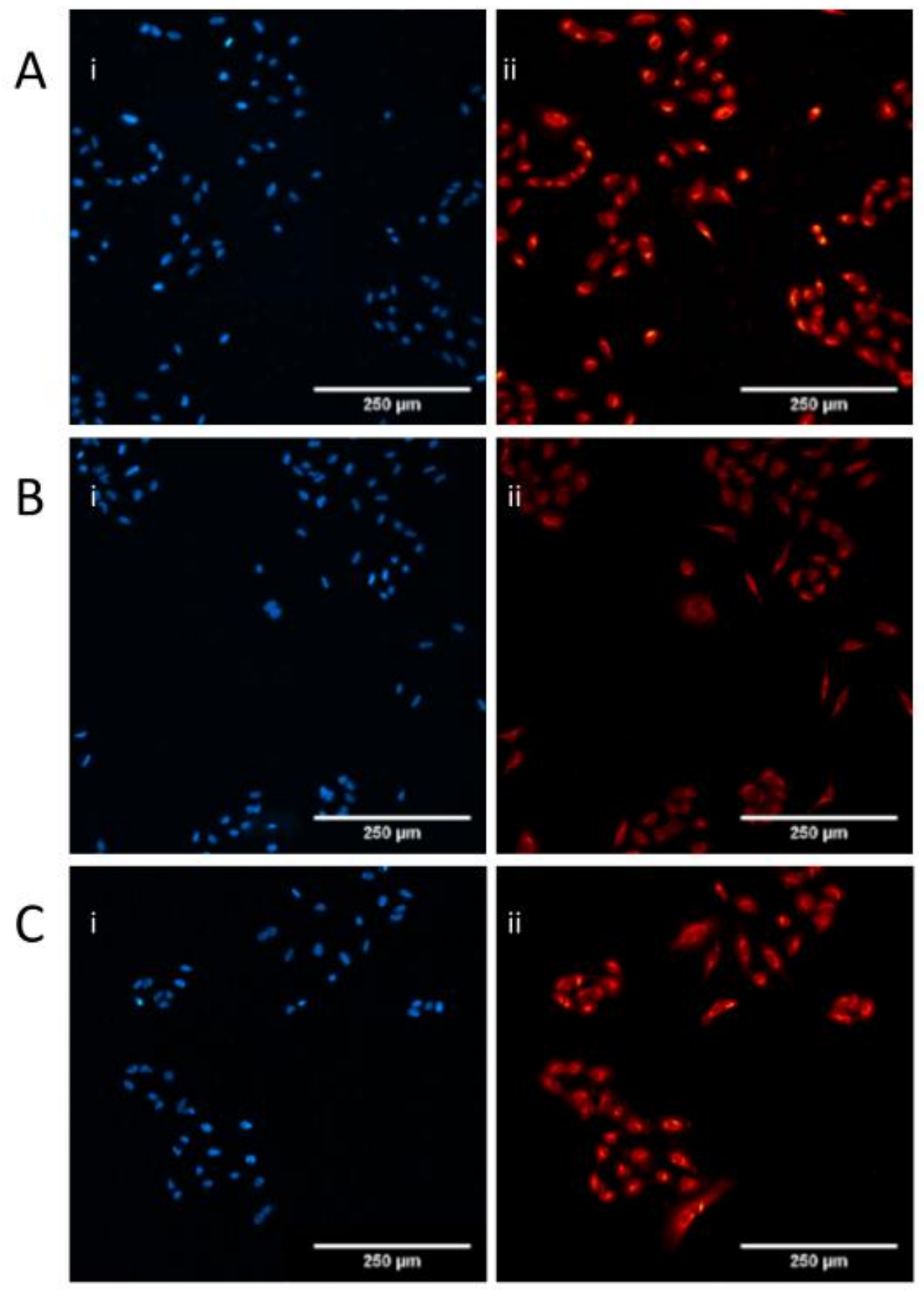

Figure 50. A549 morphology of nucleus and cell after treatment with formic acid at concentrations equivalent to the residual amounts in the $1 \mu M$ and $2 \mu M$ Discorhabdin $E$ samples.

Treatments: $A=$ Control, $B=$ formic acid (disco $E 1 \mu M$ equivalent), $C=$ formic acid (disco $E 2 \mu M$ equivalent); Stains: $i=$ DAPI - nucleus stain, $i i=$ Cell Mask Deep Red Plasma Membrane. Images were taken 48 hours after treatment incubation. A549 cells were fixed and adhered to slides, and imaged on the IN Cell Analyser 6500. Images are representative of treatment conditions from a 6-well plate, $n=4$. 


\subsection{Discussion}

The protocol established in chapter three and four is robust enough to assess the bioactivity of novel natural product compounds. This was shown by discorhabdin E flow cytometry results. From following the protocol as if the compound was novel, it was seen that the compound was active and changed the morphology of cells even at a concentration when cell death was minimal, that is, $1 \mu \mathrm{M}$. At the higher concentration of $10 \mu \mathrm{M}$, the compound was shown to be cytotoxic, with almost all cells affected, and $\sim 70 \%$ of cells dead (figure 47). However, the cytotoxicity results illustrate there was too large of a shift from testing at $1 \mu \mathrm{M}$ to $10 \mu \mathrm{M}$. A better approach would be to retest at 10, 7.5, 5 and $2.5 \mu \mathrm{M}$. This would provide more suitable concentration intervals which would increase the likelihood of finding the Goldilocks concentration.

Interestingly, the flow cytometry morphology results (figure 48) imply bioactivity is occurring $<1 \mu \mathrm{M}$. The cell size is minimally different from the control, but cell granularity increased at this treatment concentration. In both the $1 \mu \mathrm{M}$ and $10 \mu \mathrm{M}$ treatment data, there is a population with low forward scatter, PI negative, indicating that cells were not dead, but rather just small. Combined with the increased SSC, these results are suggestive of autophagy. ${ }^{101}$ Autophagy is a cellular process which occurs as a result of cellular stress. Specifically, the following events happen: (1) the development of phagophores, (2) the envelopment of intracellular contents to form an autophagosome which then (3) becomes fused with a lysosome. ${ }^{101-105}$ The enzymes in the lysosome digest the intracellular contents from the autophagosome. The formation of lots of vesicles such as the autophagosome and lysosomes would ultimately increase the cell granularity as seen by flow cytometry results (figure 48). Autophagy is commonly said to lead to cell death as it occurs as a result of cellular stress, however, successful autophagy occurs to prevent cell death. In certain circumstances the cell has cell signalling pathways which lead to autophagic responses that allow the cell to survive under these stressful conditions, for example, under hypoxic conditions when cells do not have enough oxygen to respire. To cope with the lack of oxygen, a cell signalling pathway with HIF-1 is activated to promote angiogenesis, erythropoiesis and reduce mitochondrial biogenesis and respiration. ${ }^{104}$ Specifically, this pathway clears mitochondria by a specific autophagic process called mitophagy to allow the cell to continue to survive without respiration. It is commonly seen in the hypoxic environments of tumours. 
The main known target of the discorhabdin family is HIF-1, specifically inhibition of HIF$1 \boldsymbol{\alpha} .^{106,107}$ As previously mentioned, this pathway is involved in regulation of mitophagy. This reveals that the autophagic response hypothesised from figures $48 \& 49$ is consistent with the target discorhabdins are reported to have. ${ }^{107,108}$ Ultimately, this strengthens the argument that autophagy occurred. Further experimentation is required to validate this observation.

These results highlight a potential issue. Autophagy could be induced by the direct effect of a compound or an indirect result of cells being highly stressed. ${ }^{104}$ Thus, it poses the question whether the autophagy seen by discorhabdin $\mathrm{E}$ is a an effect from the compound targeting a cell signalling pathway which induces autophagy or a cytotoxic drug response. Further tests of discorhabdin E treatment at more concentration intervals between $1 \mu \mathrm{M}$ and $10 \mu \mathrm{M}$, such as the ones suggested above and other cytotoxic drugs may be able to distinguish this.

Lastly, as suggested in the isolation results, a new compound, which had a corresponding ion detected at $m / z 521$ was potentially found by mass spectrometry. A future experiment could be conducted to elucidate the structure and confirm if it is novel.

\subsection{Conclusion}

This chapter exemplified the flow cytometry cytotoxicity and morphology assay protocol created in chapter three, together with images of the cells is effective at suggesting the compound is bioactive. Issues with the original assay rules with the initial concentration cytotoxicity tests arose. The cytotoxicity results for discorhabdin E suggested minor alterations are needed to include testing at more concentrations between $1 \mu \mathrm{M}$ and $10 \mu \mathrm{M}$. This would hopefully allow more dynamic results on a novel compound's cytotoxicity so a stronger representative cell morphology of the compound's bioactivity can be achieved, and not estimated. However, the very purpose of the assay was still met despite this. The aim was to assess the bioactivity of any novel compound, whatever its bioactivity may be - this was accomplished at the concentration chosen to carry out cell morphology analysis for discorhabdin E. 
Chapter 6: Final Discussion 


\subsection{Semi-quantitative approach established}

This project set up the foundations for a new pipeline for screening novel compounds from the natural products laboratory at Te Herenga Waka. It has provided an alternative, more informative approach than the simple cytotoxicity screen commonly carried out. The semiquantitative flow cytometry assay which was initially proposed to find a "Goldilocks" concentration for a MCP, together with fluorescent stained images, is a useful tool alone. This is because it showed it can deduce the presence of bioactivity of compounds without the need for full quantitative MCP analysis and the acquisition or construction of a morphobase. The flow cytometry cytotoxicity data suggested when a drug had a cytotoxic effect, if any, and the flow cytometry morphology data effectively supplemented it, by highlighting changes in morphology before the compound became notably cytotoxic. Images of A549 cells also provided additional information about morphological changes that could not be provided by the two morphological parameters, cell size and granularity, measured by flow cytometry. That is, information about the other cellular components, such as nuclei morphology, and also cell roundness and protrusions.

It is well within the scope of this screening technique going forward to stain with additional fluorescent dyes for other intracellular organelles to gain more information about how compounds affect the morphology of specific organelles. This would begin to suggest potential targets for compounds, and mechanism of action. Previous literature which established a cell painting assay for this purpose has demonstrated this. ${ }^{60-62}$ Further, this cell painting assay was used for a full quantitative MCP assay.

\subsection{Feasibility of a quantitative MCP approach}

This project demonstrated the feasibility of currently establishing an MCP at Te Herenga Waka. As aforementioned, fluorescently stained images were obtained and illustrated that compounds changed the morphology of cells, at a level detectable by the human eye. This phenomenon can be measured quantitatively. There is a small number of publications which detail how software packages can identify and segment cellular objects and then quantify parameters from these objects, such as size, roundness and ellipticity. If many drugs with known targets are put through this protocol, the quantified data can then be collated to form a morphobase. Ultimately, it establishes a screening technique known as an MCP to deduce the target of drug candidates with unknown targets. Additionally, a full MCP assay removes both 
technical and human bias completely. Thus, the applicability of this assay would be very useful for academic laboratories.

As discovered in chapter four, there are a number of skill requirements necessary to establish and carry out an MCP, which collectively make the approach infeasible for one person alone. For example, researchers must know how to carry out flow cytometry, operate highthroughput microscopes, use multiple software and machine learning packages that require heavy computational processing power, and analyse and visualize the very large datasets that result.

As highlighted by this project, the computational skills required to carry out many of these processes is large. One example of this is the competency required to process the thousands of images on CellProfiler using a computer such as a high performance computing cluster. As discussed in chapter four, multiple efforts with different computers, image file numbers inputted, and different CellProfiler setting parameters were attempted, but all errored and made it infeasible without computing power higher than the ones used here.

It also should not be understated the skill required to operate CellProfiler. It is likely further optimisation will need to occur to the pipeline as different staining techniques or imaging on different days also impacted on segmentation quality. For this reason, a researcher(s) in the future will need to have or gain experience on how to operate the software. Without it the underlying rationale for the use of each module is lost. For example, the software needs to read the fluorescent intensity of one stain at a time in order to identify cellular objects, but image files do not necessarily come with the channels split. To do this, the ColorToGray module was inserted to split the channels into separate images - as the module title does not allude to its function, specialist knowledge about the module would not have been discovered without other researchers with expertise in the software.

\subsection{Future directions}

As a consequence of assessing the feasibility of this approach, the foundations to implement this assay were established in chapter four. Thus, in the future, there is the opportunity to create a full quantitative MCP. Many of the protocol described in this project will apply to an MCP. For example, the optimised CellProfiler pipeline, which microscope is best, which stains should be used to segment cells, code to transform file names from the IN Cell Analyser 6500 so CellProfiler can read its metadata, code to observe the accuracy of the 
morphological data output from CellProfiler as boxplots, and a CellProfiler pipeline for image quality control to improve accuracy was established.

\subsection{Concluding remarks}

Improved characterisation of novel natural product compounds will increase the chance of discovering an approval worthy drug. The results from this thesis augment a morphological approach within the drug discovery and development pipeline. Further, an extension into a morphological cell profiling approach will lead to rapid target identification of novel compounds. In doing so it further condenses the early stages of the drug discovery and development pipeline, thus overall contributing to a reduction in the time and resources required to assess drug candidates at an institutional level. 


\section{Appendix}

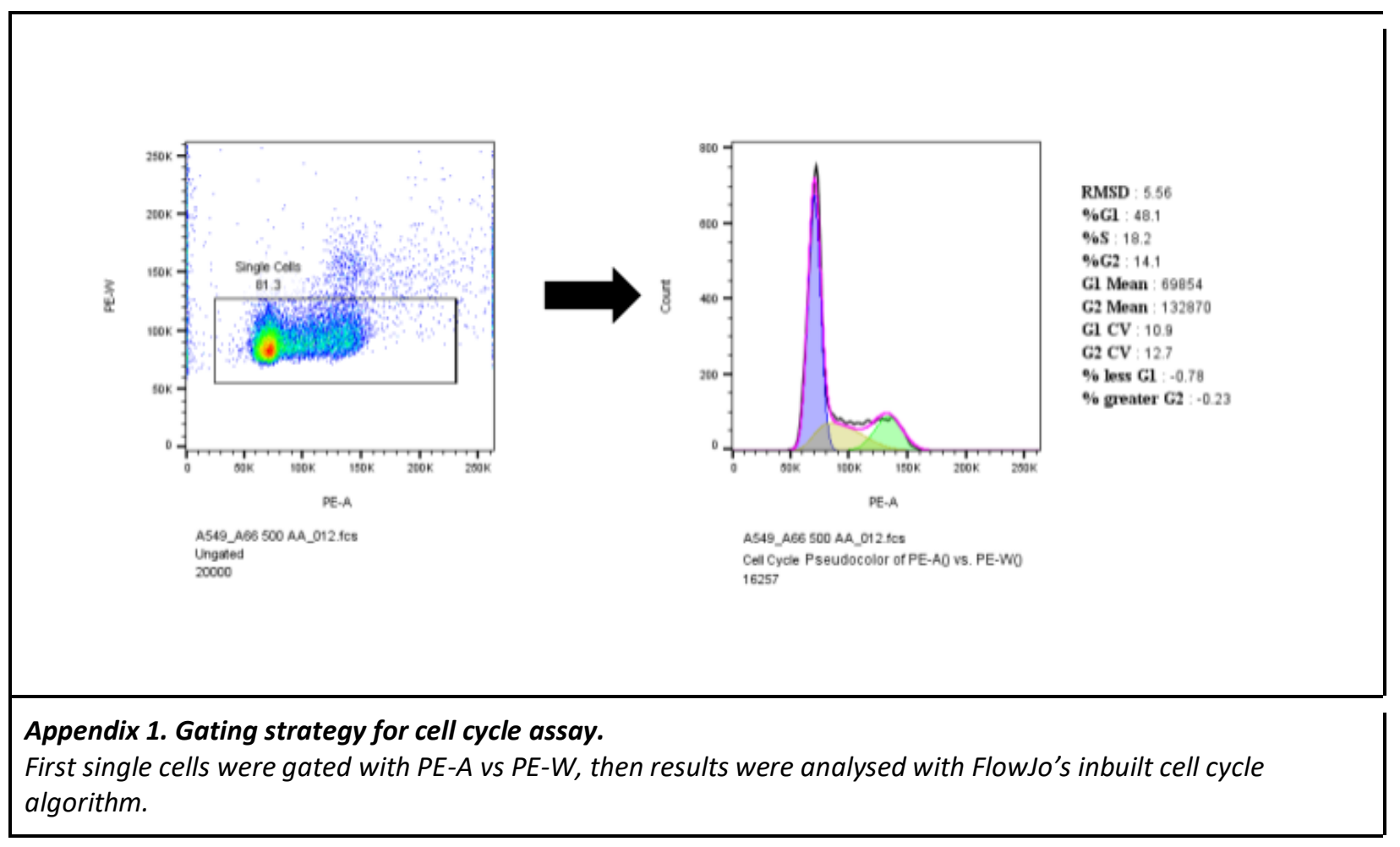




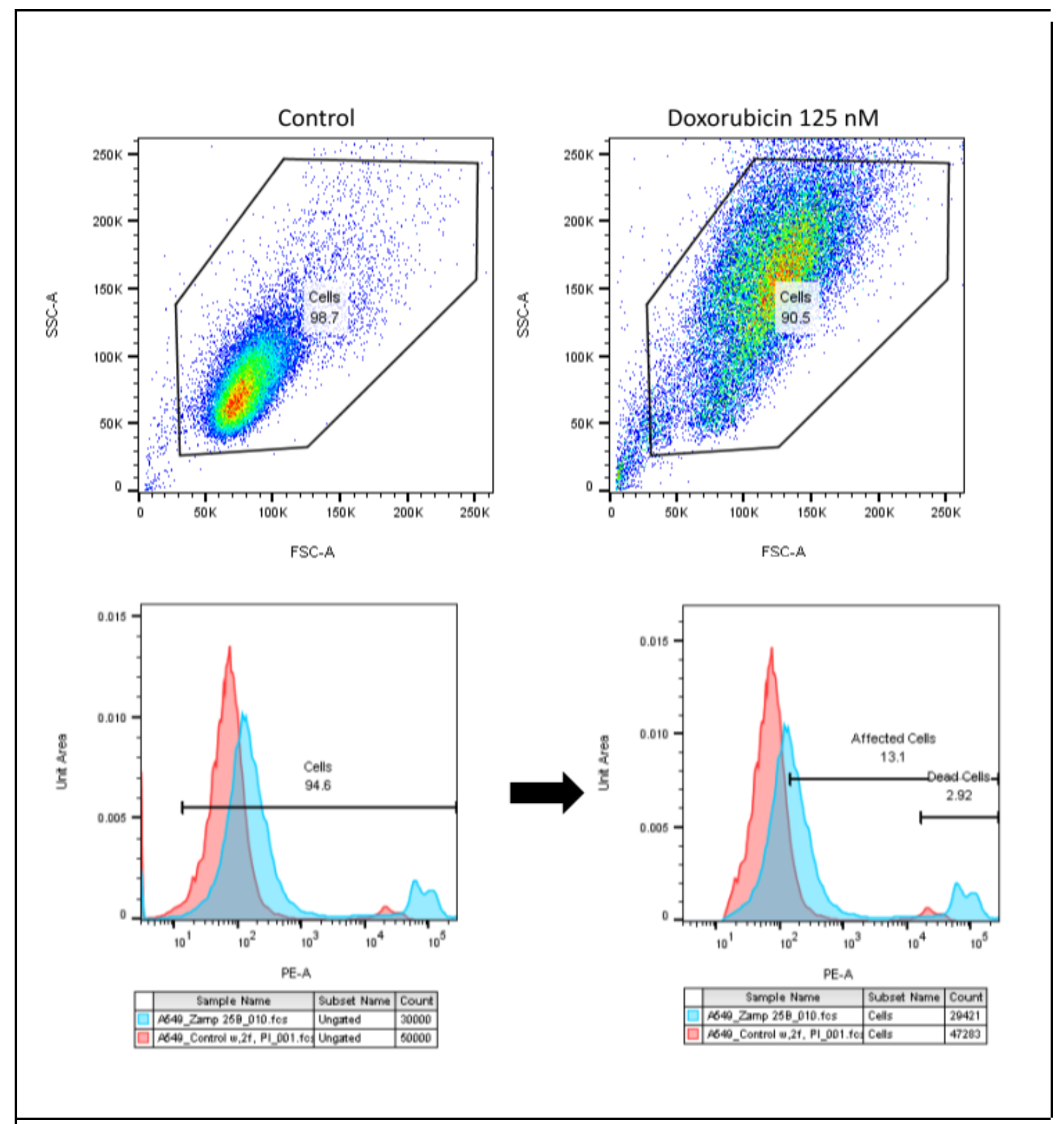

Appendix 2. Gating strategy for cytotoxicity assay.

Top = how cells were gated for, exemplified in control and doxorubicin $125 \mathrm{nM}$ treatment samples.

Bottom = Gating strategy schematic. First cells were gated for to exclude debris, then affected and dead cells were gated based on PE-A intensity against a control 


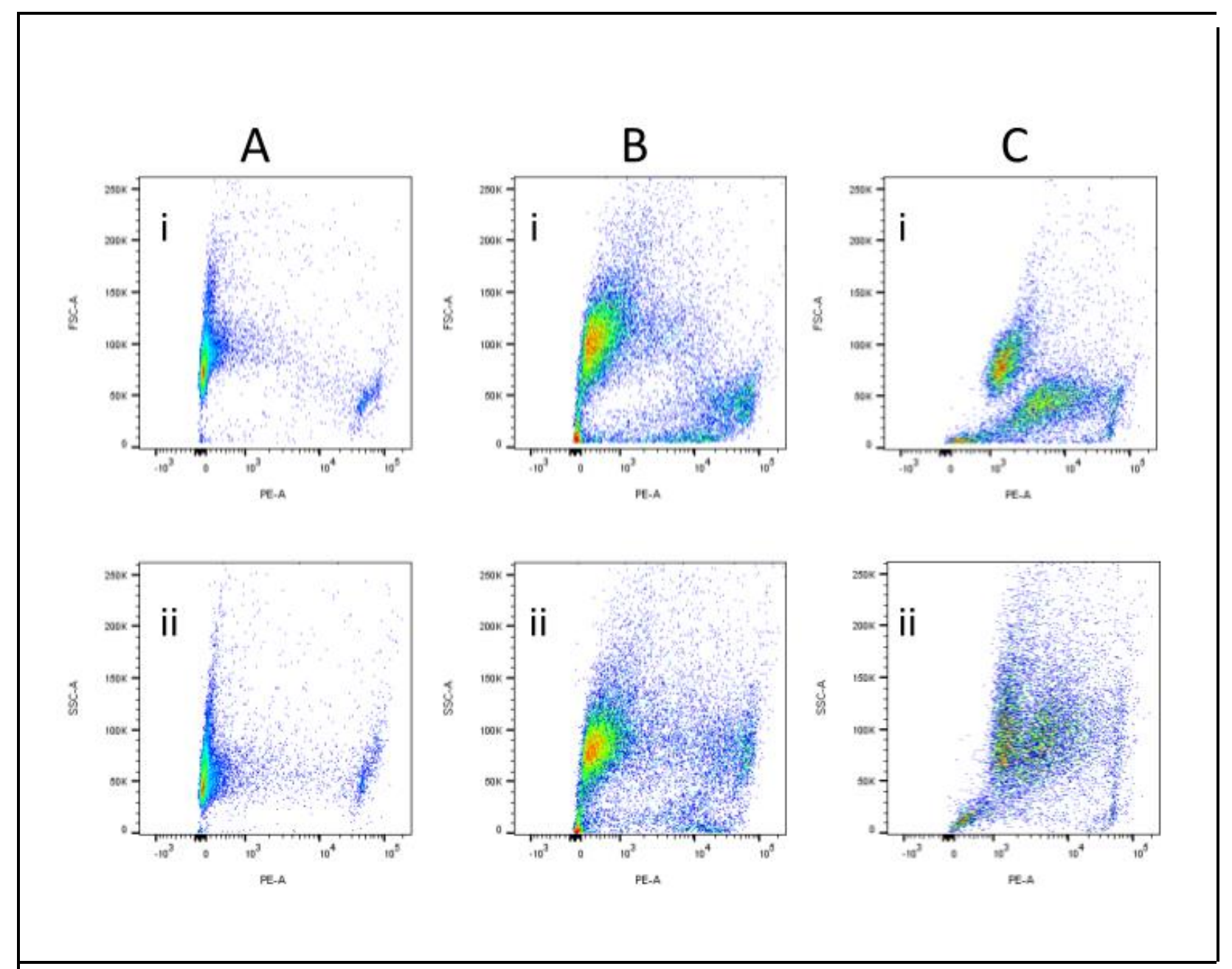

Appendix 3. Morphology parameters compared to PI content to understand how cytotoxicity related to morphological change.

$A=$ untreated, $B=$ Taxol $(1 \mu M), C=$ doxorubicin $(1 \mu M) . i=$ Forward scatter (FSC) vs PE- $A$ (PI content), ii = side scatter (SSC) vs PE-A. Figures show a low forward scatter has a higher proportion of dead cells, but a low side scatter does not correlate to a higher proportion of dead cells. 
$x=$ which(names(laurendataset)=="AreaShape_Area") \#This is where the graphs will start from

$y=$ ncol(laurendataset) \#Number of columns it will run to

$v=$ which(names(laurendataset)=="Metadata_Drug...6")

$\mathrm{s}=$ paste(laurendataset[[1,v]], "', sep = " ") \#This gets the name of the drug you're looking at

laurendataset\$Metadata_Dose....3 <- as.character(laurendataset\$Metadata_Dose...3)

laurendataset\$Metadata_Dose...3<- factor(laurendataset\$Metadata_Dose...3, levels = c("0", "15", "31", "62", "125", "250"))

\#This sorts them into numeric order

for(i in 14:ncol(laurendataset)-3) \{

yvar $=$ names $($ laurendataset $)[i]$

plotname <- trimws(gsub("AreaShape_", s, yvar))

my_col <- sym(names(laurendataset)[i])

p1 <- eval(substitute(

ggplot(laurendataset, aes(x= Metadata_Dose...3,y=!!my_col, fill=Metadata_Dose...3)) +

+ geom_boxplot(aes(fill=Metadata_Dose...3), alpha=1) + scale_x_discrete(name = "'") + ggtitle(plotname) + theme $($ plot.title $=$ element_text $($ face $=$ "bold" $))+$ theme $($ plot.title $=$ element_text $($ hjust $=0.5))$

+ theme(legend.position="bottom") +

theme $($ legend.title = element_blank ()$)+$ scale_y_discrete $($ name $=$ "')

,list( $(\mathrm{i}=\mathrm{i})))$

$\operatorname{print}(p 1)$

myplots[[i]] <- p1

for(i in 1:length(myplots))

\{

file_name = paste(s,"_", myplots[[i]]\$labels\$y, ".tiff", sep="")

tiff(file_name)

$\operatorname{print}($ myplots[[i]])

dev.off()

\}

Appendix 4. Code made by Danyl MacLaughlan for RStudio to transform CellProfiler quantified morphology data spreadsheet into box plots. 
\# Script to change names of InCell $6500 \mathrm{HS}$ image names

\# Need to set working directory to source

\# Defining the experimental prefix

prefix <- 'Experiment34B001'

key <- read.csv('key_real2.csv',header $=$ TRUE, sep $=$ ",", as.is = !StringAsFactors)

for (row in 1:nrow(key))\{

\# Defining everything from the key

drug <- key[row, 'Drug']

folder <- file.path(getwd(), key[row, 'Folder'])

slide.number <- as.character(key[row, 'Slide'])

slide.position <- paste0('A - ', slide.number)

\# Creating folder to save new images to

save.dir <- file.path(folder, 'Renamed8')

print(folder)

dir.create(save.dir, showWarnings $=$ FALSE)

\# Getting list of all the images in a folder based on the slide

images <- list.files $($ folder, pattern = c(slide.position, '.tif' $))$

\# Looping over each image to change the name

for (image in images)\{

\# print(image)

\# Changing the laser and channel name

if (grepl('405 - Blue', image))\{

channel <- 'Blu'

\}

else\{ 


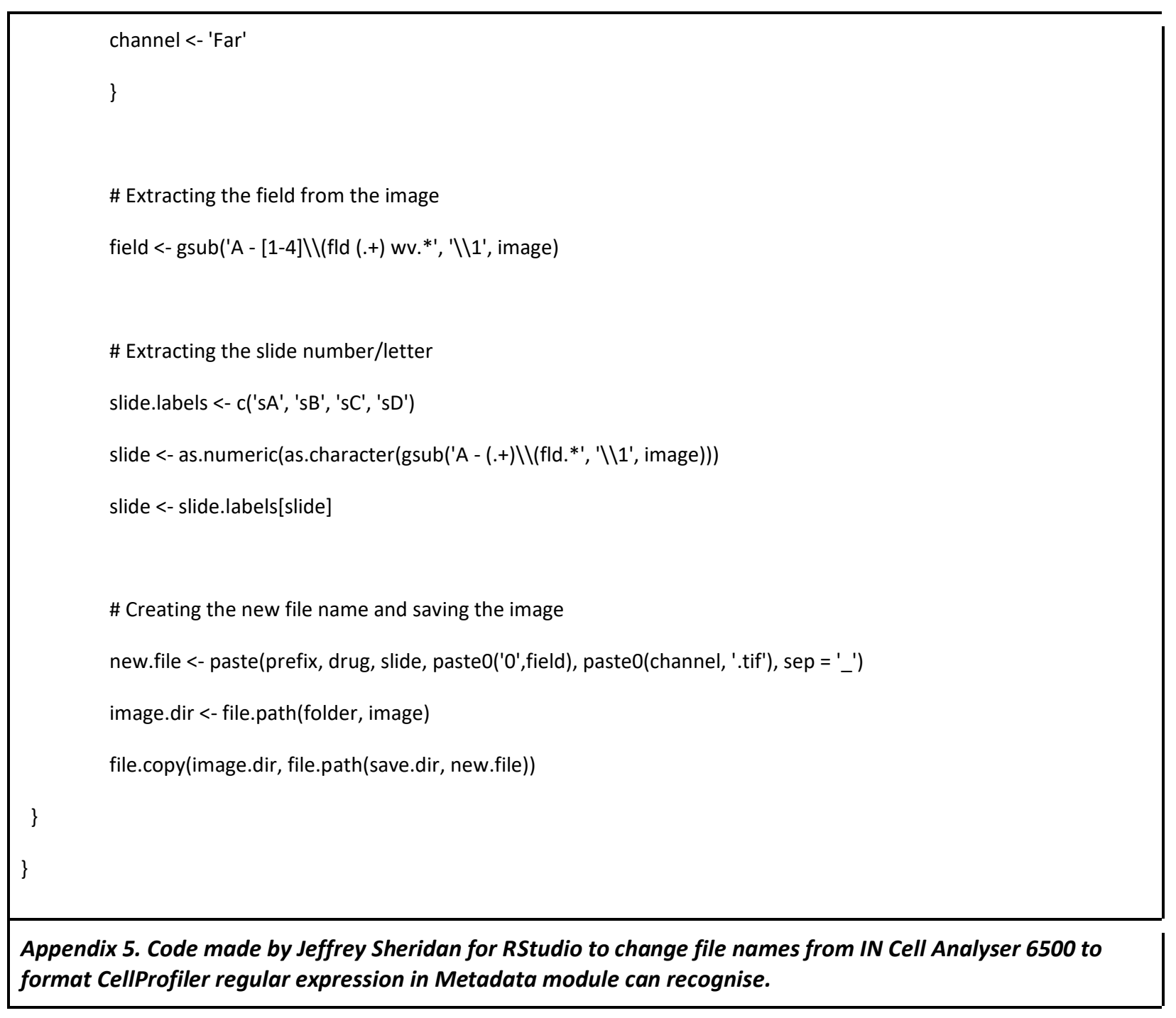




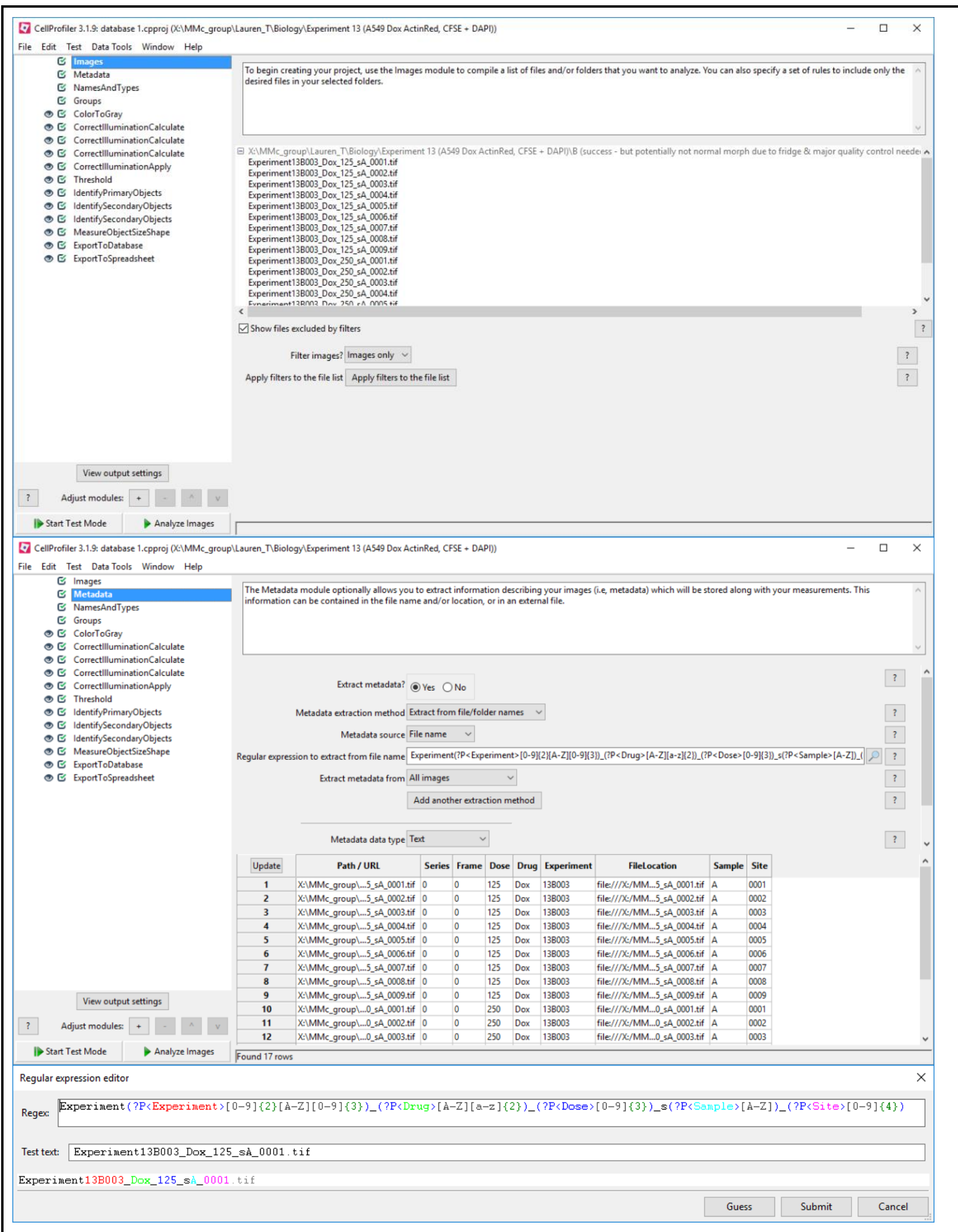




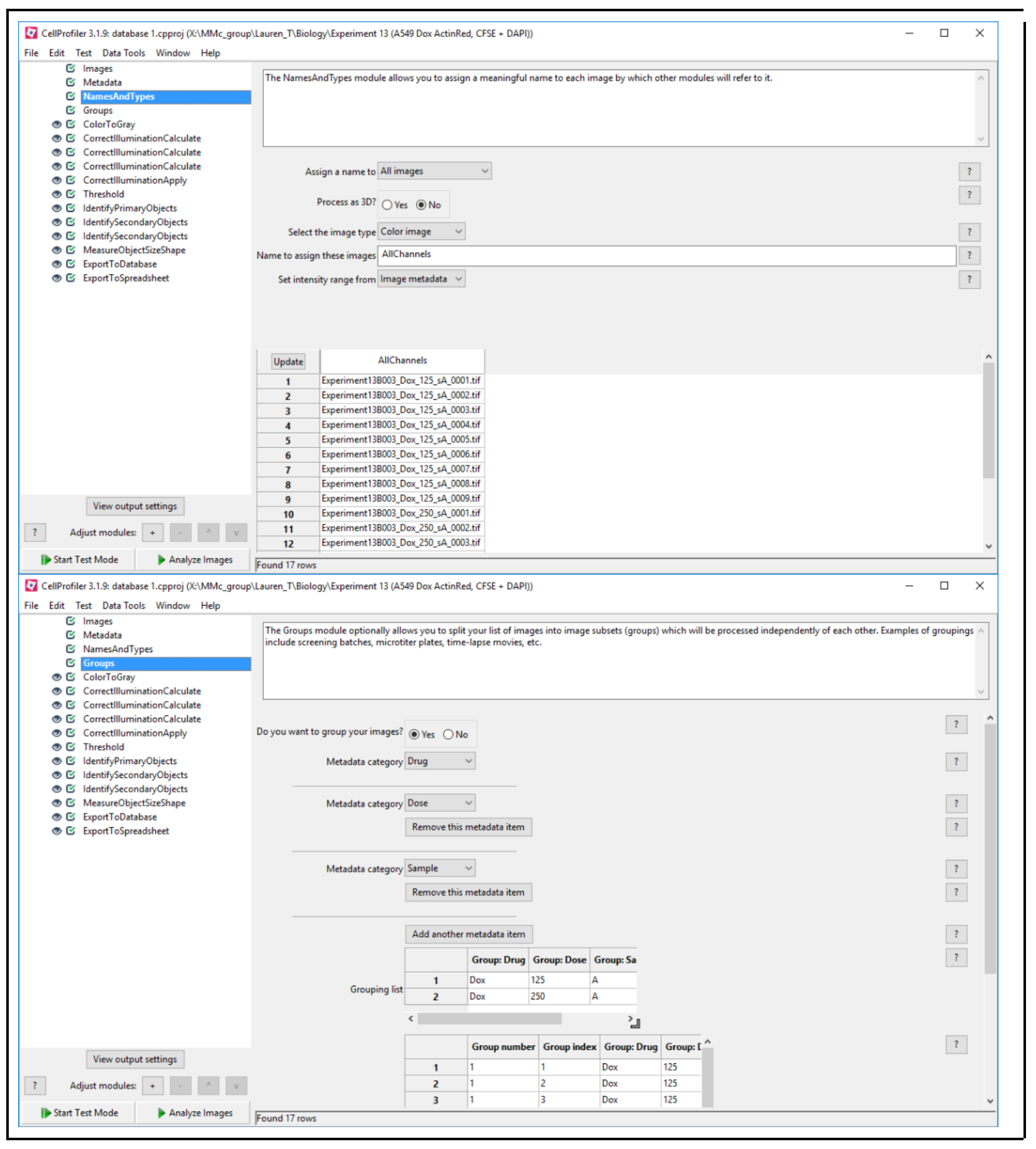




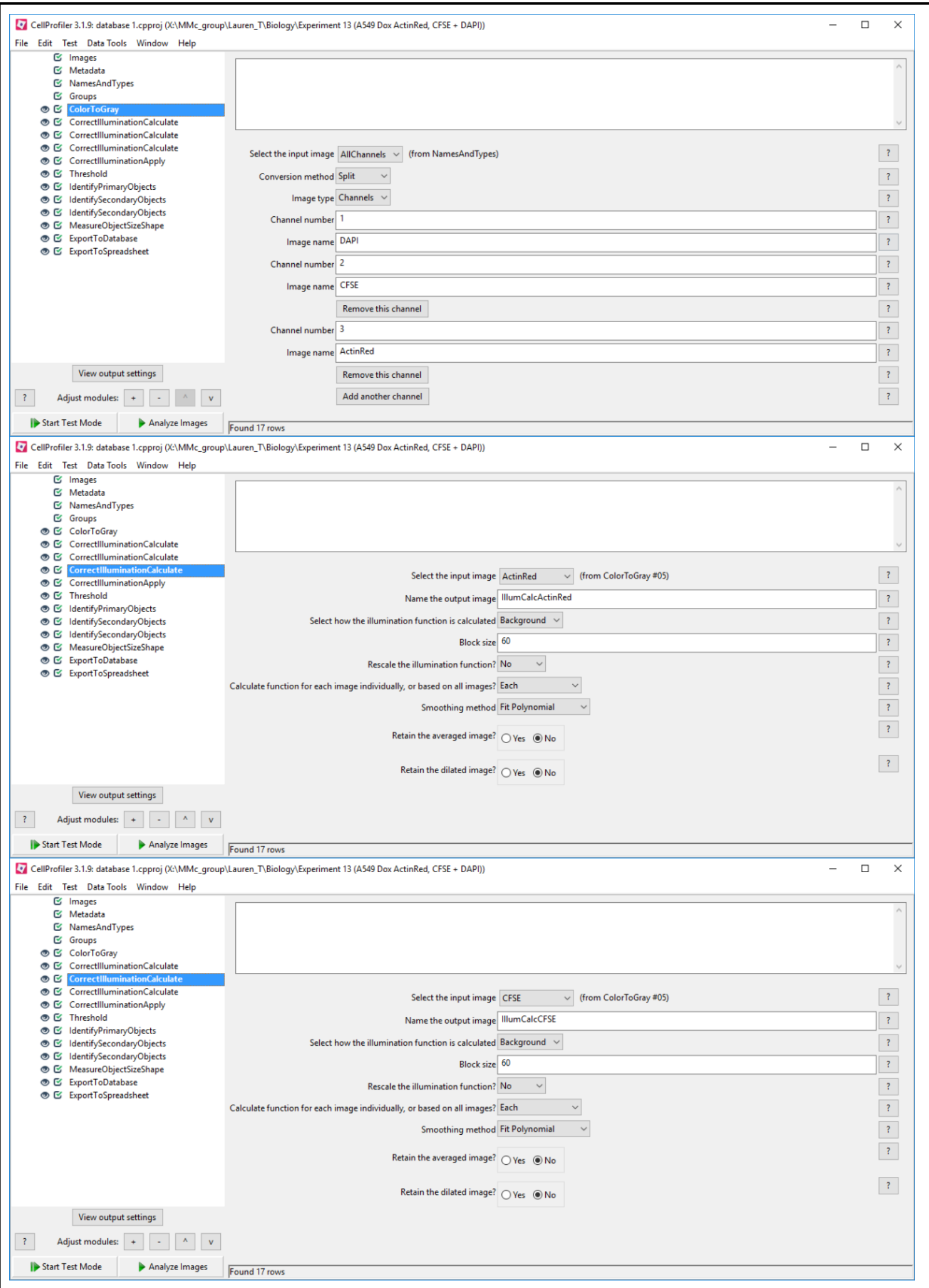




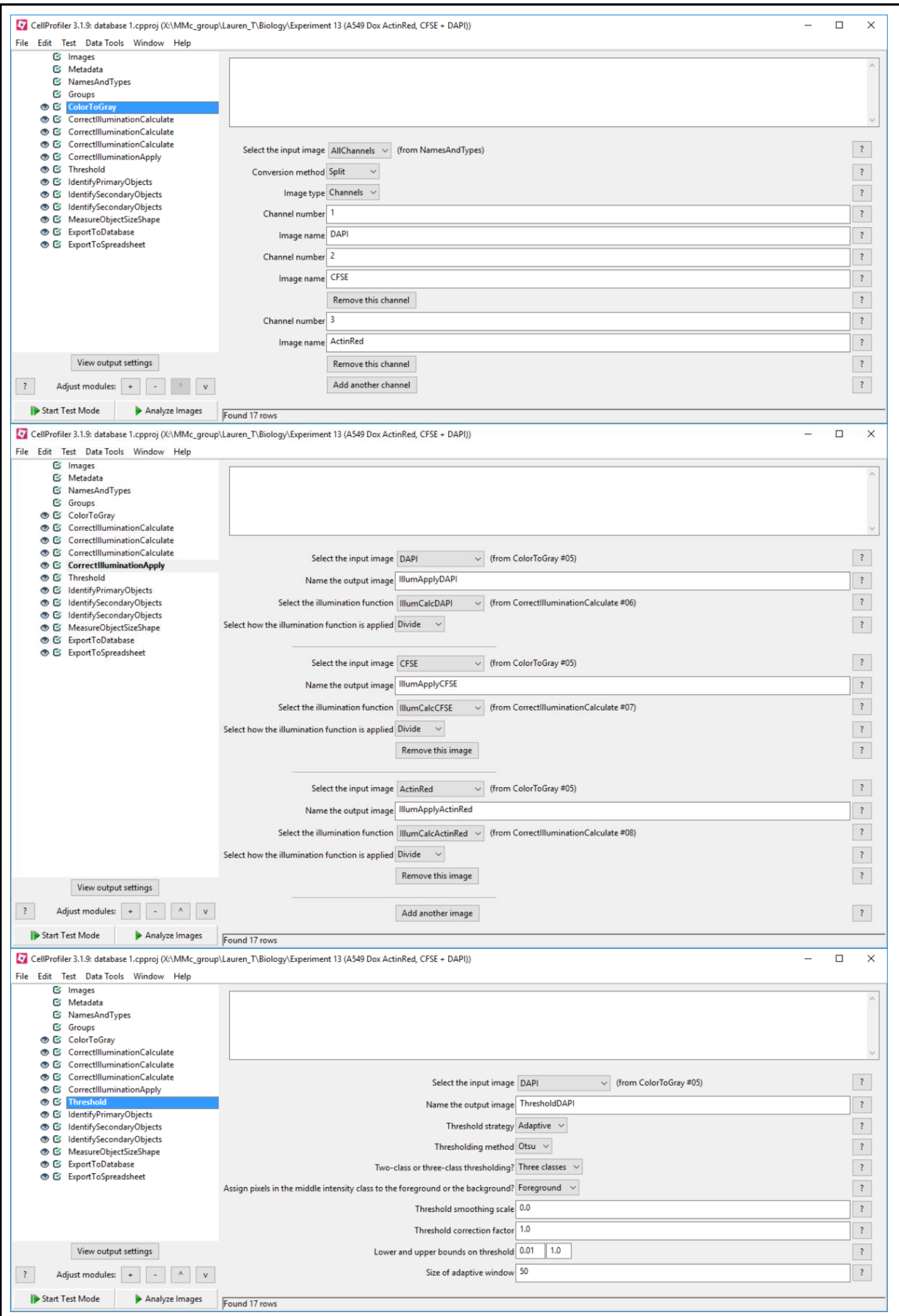




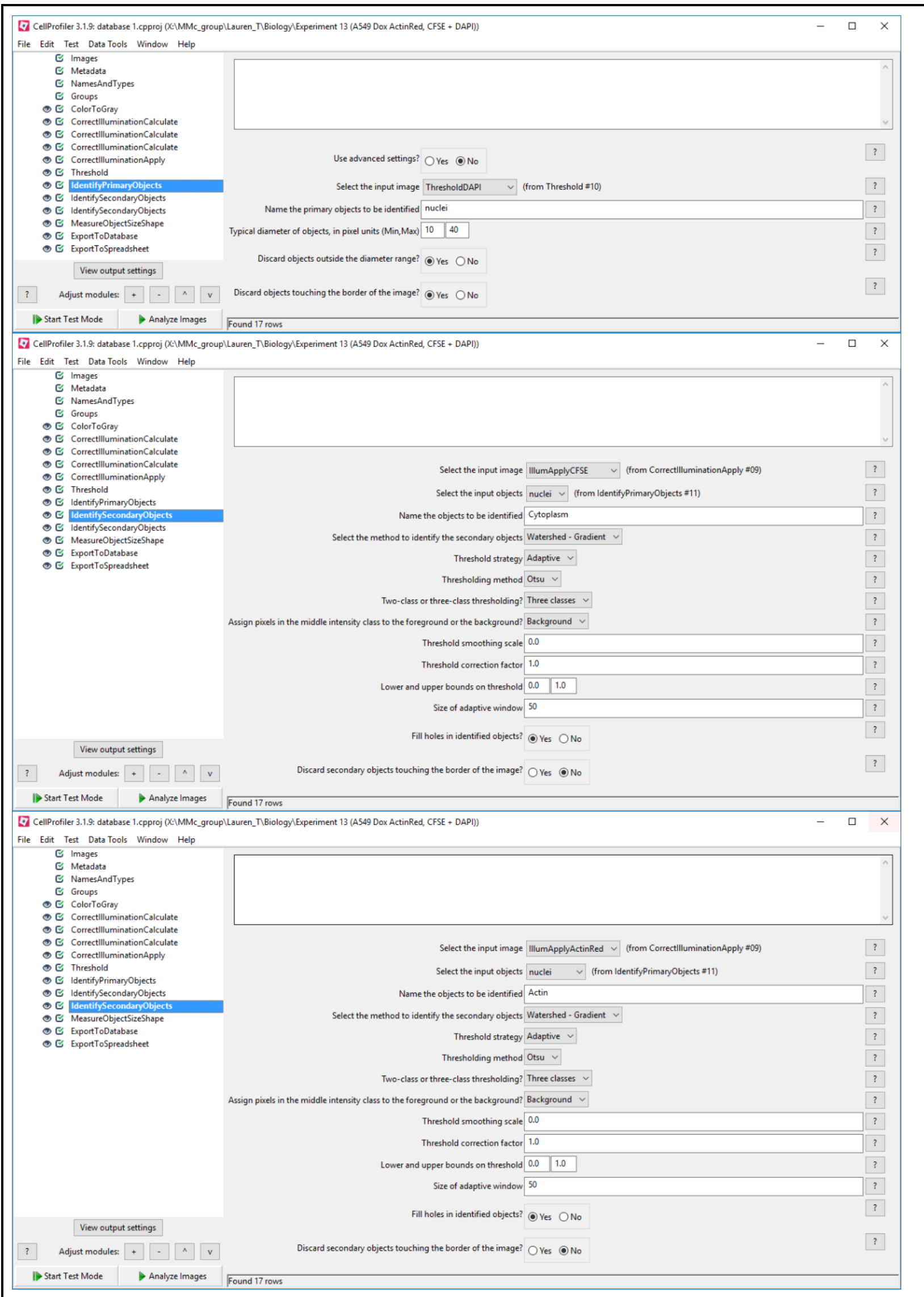




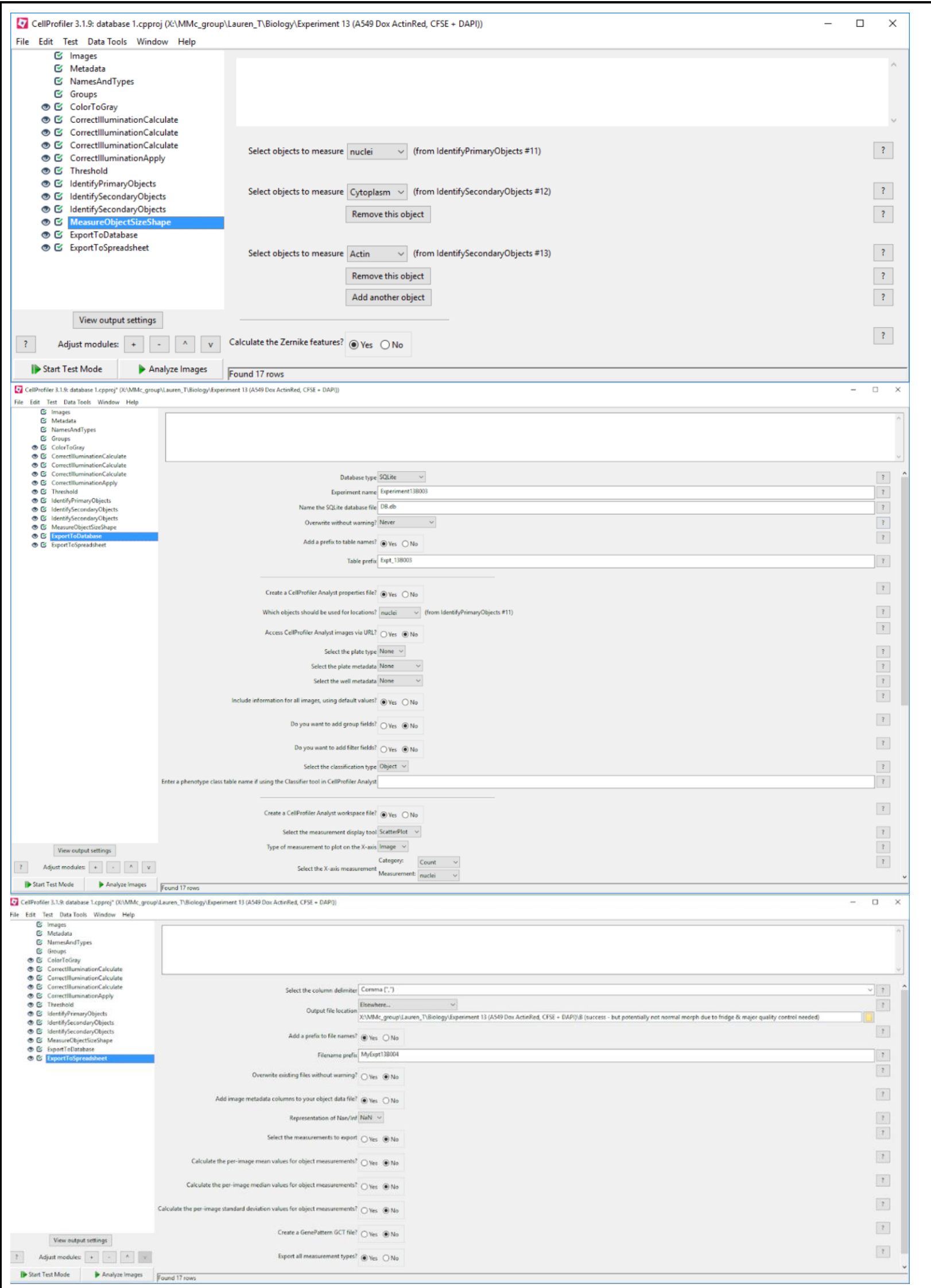

Appendix 6. CellProfiler module parameters for morphology quantification. 


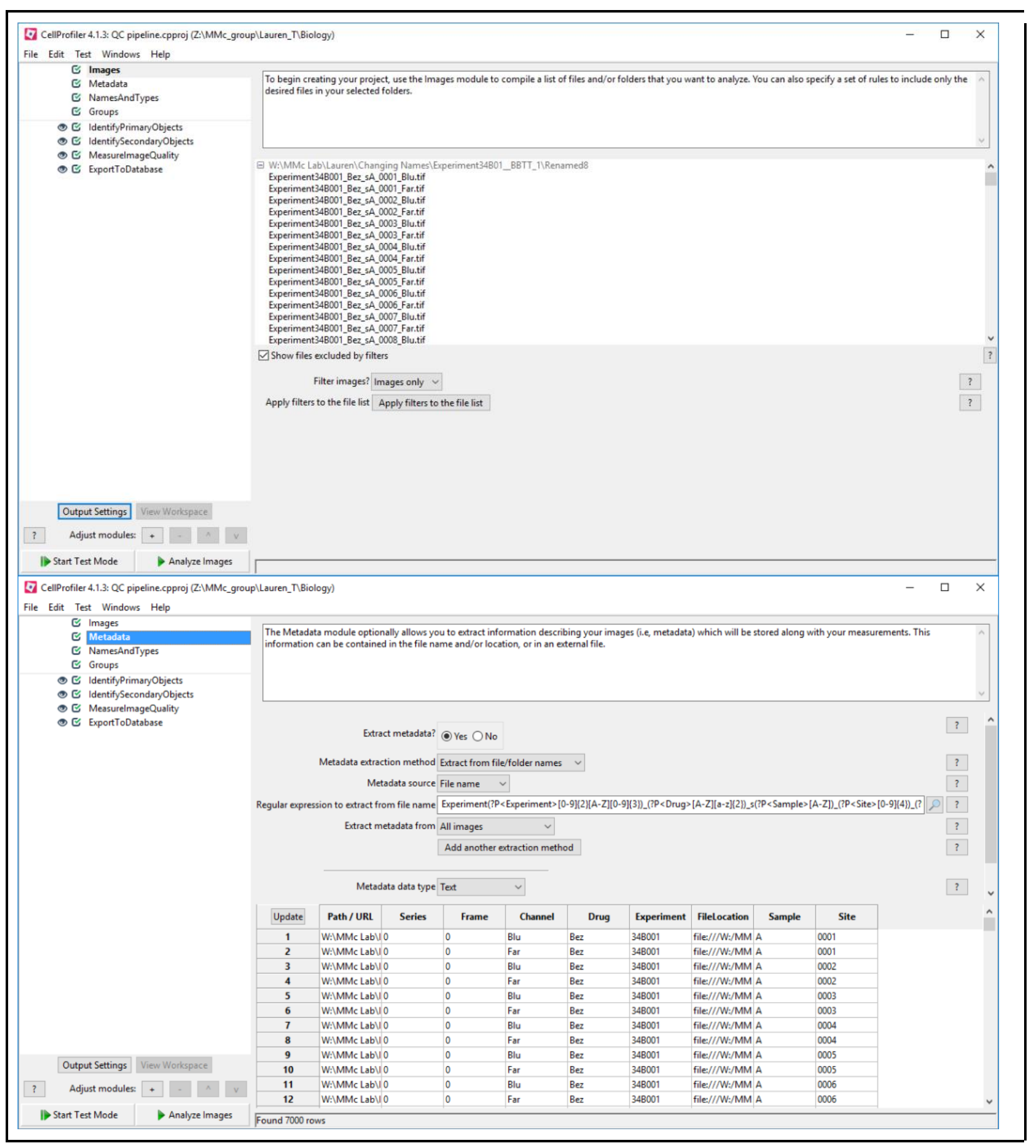




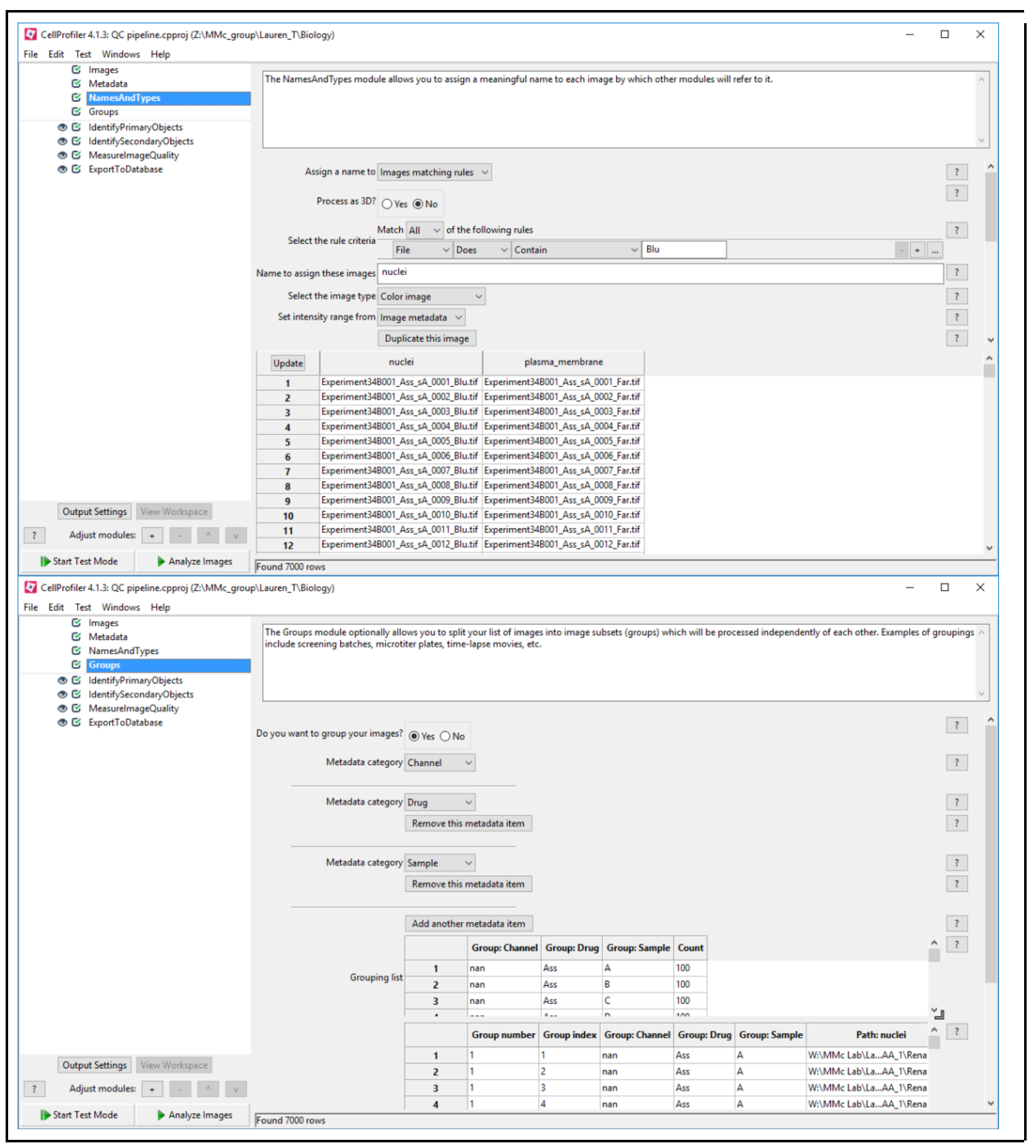




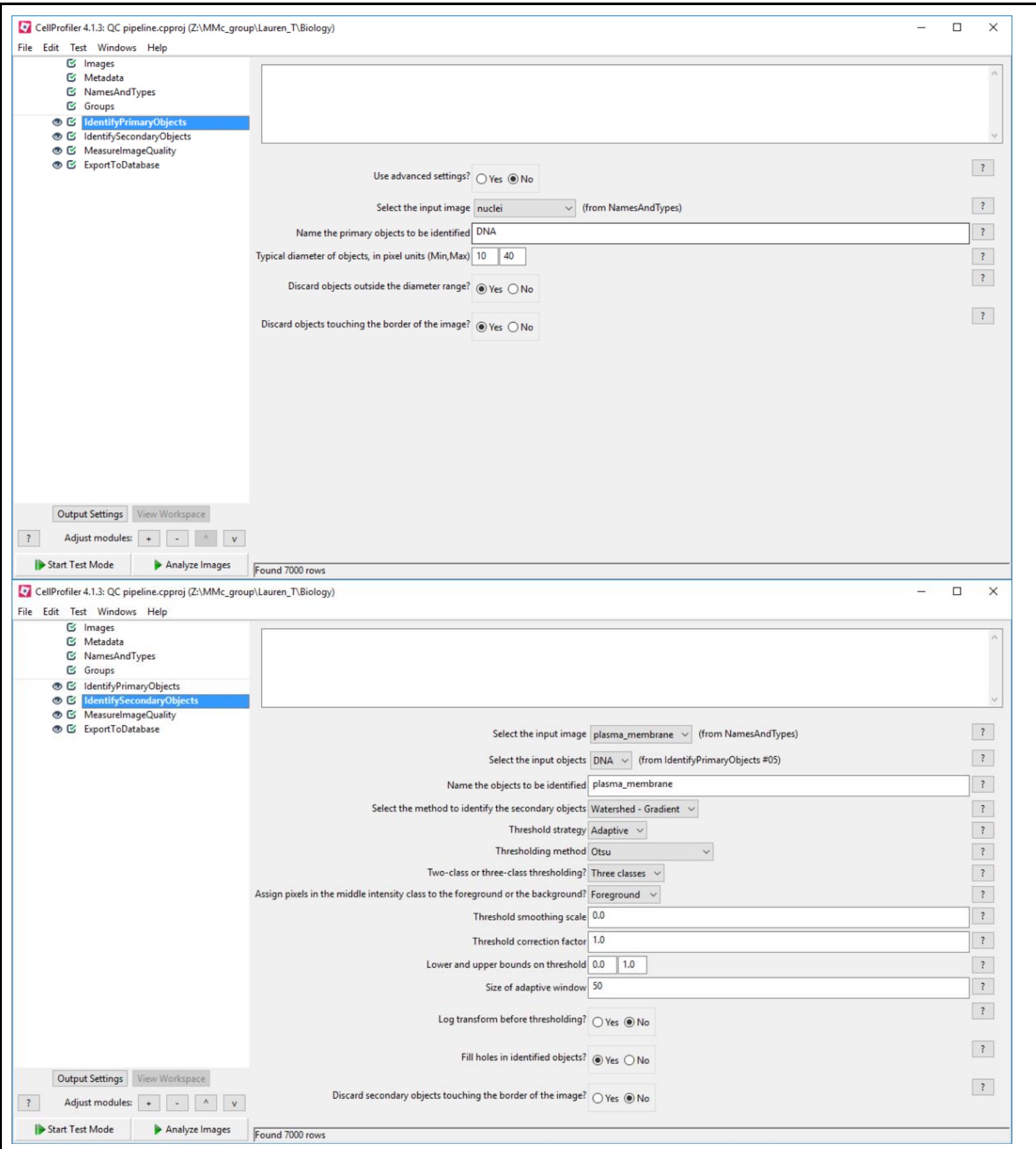




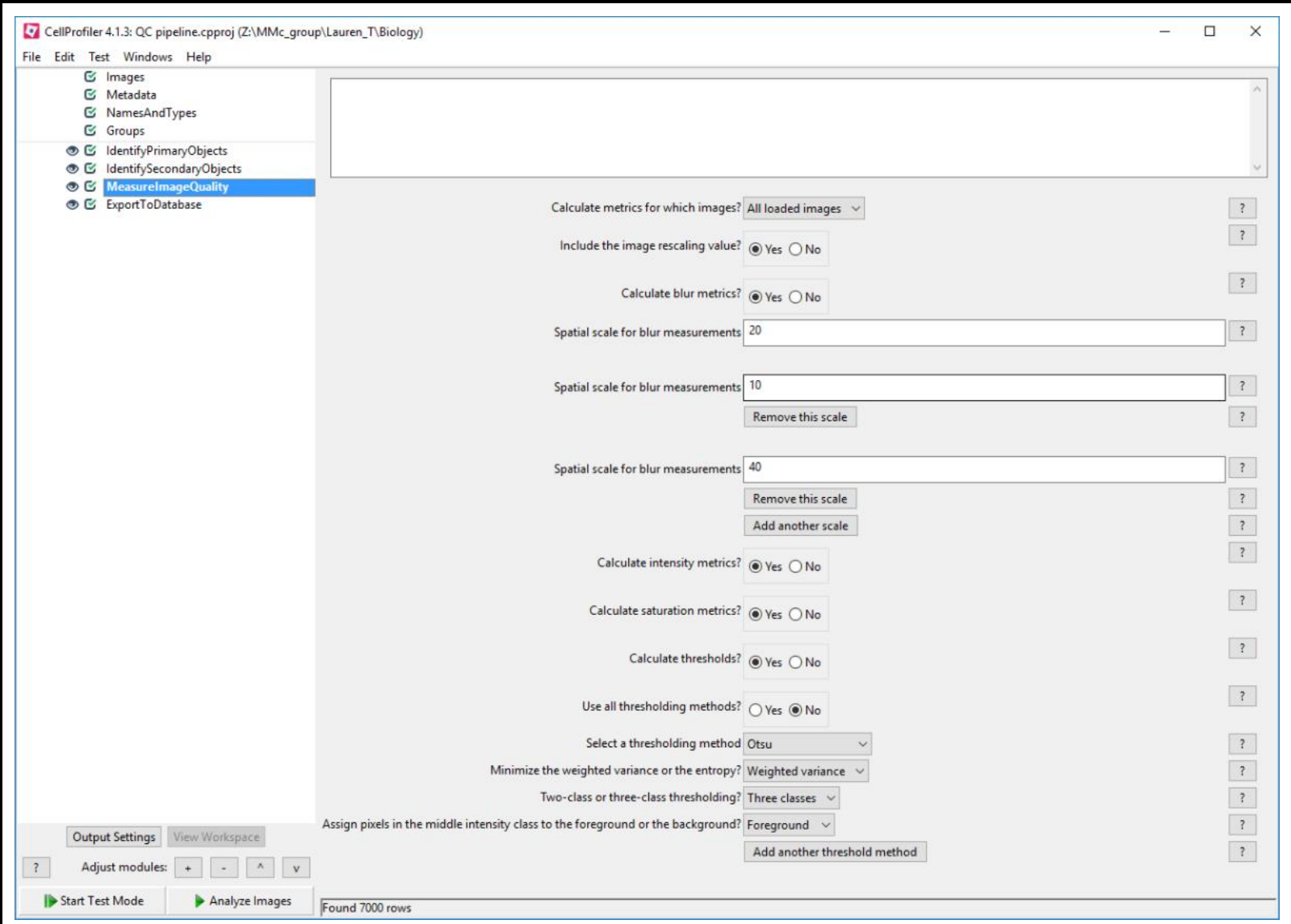




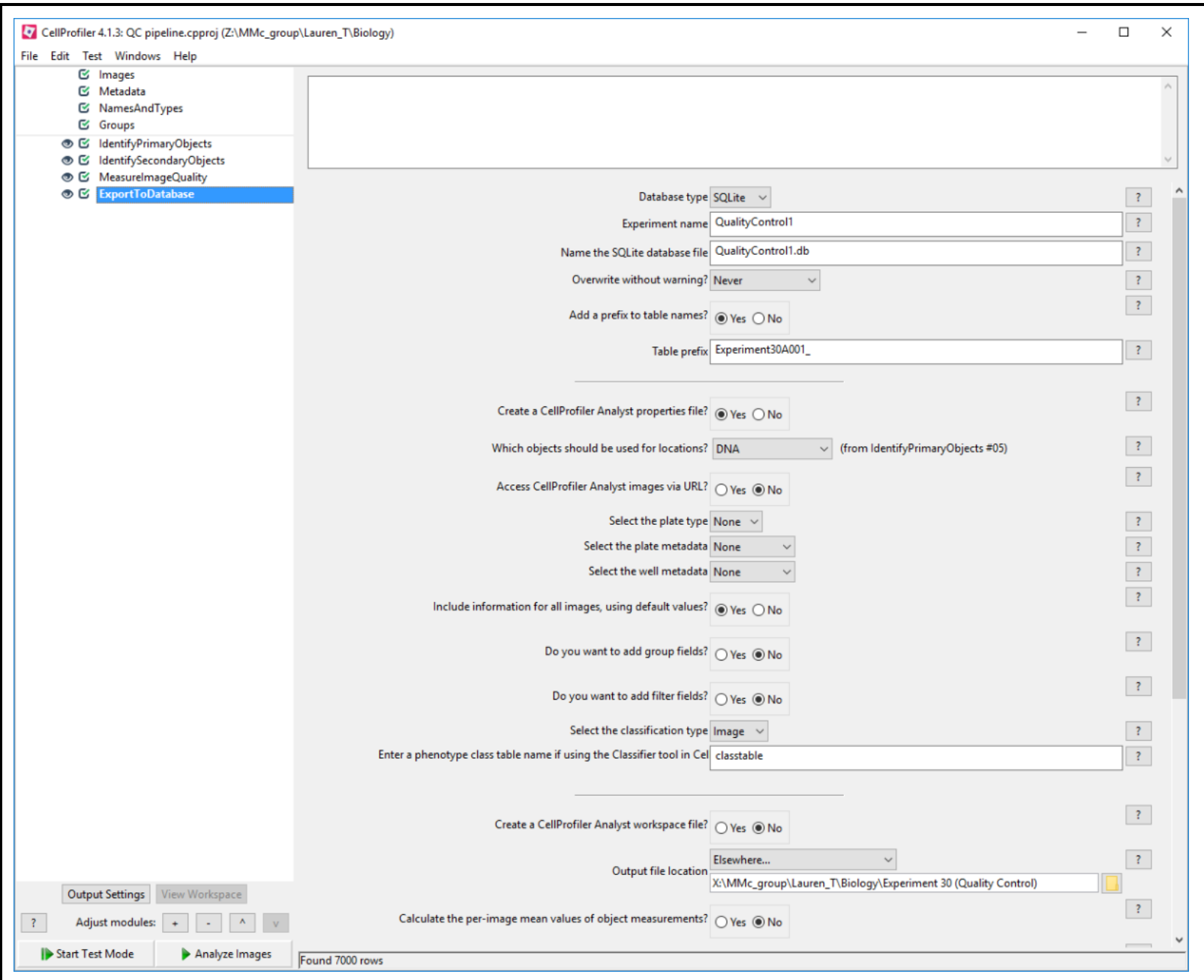




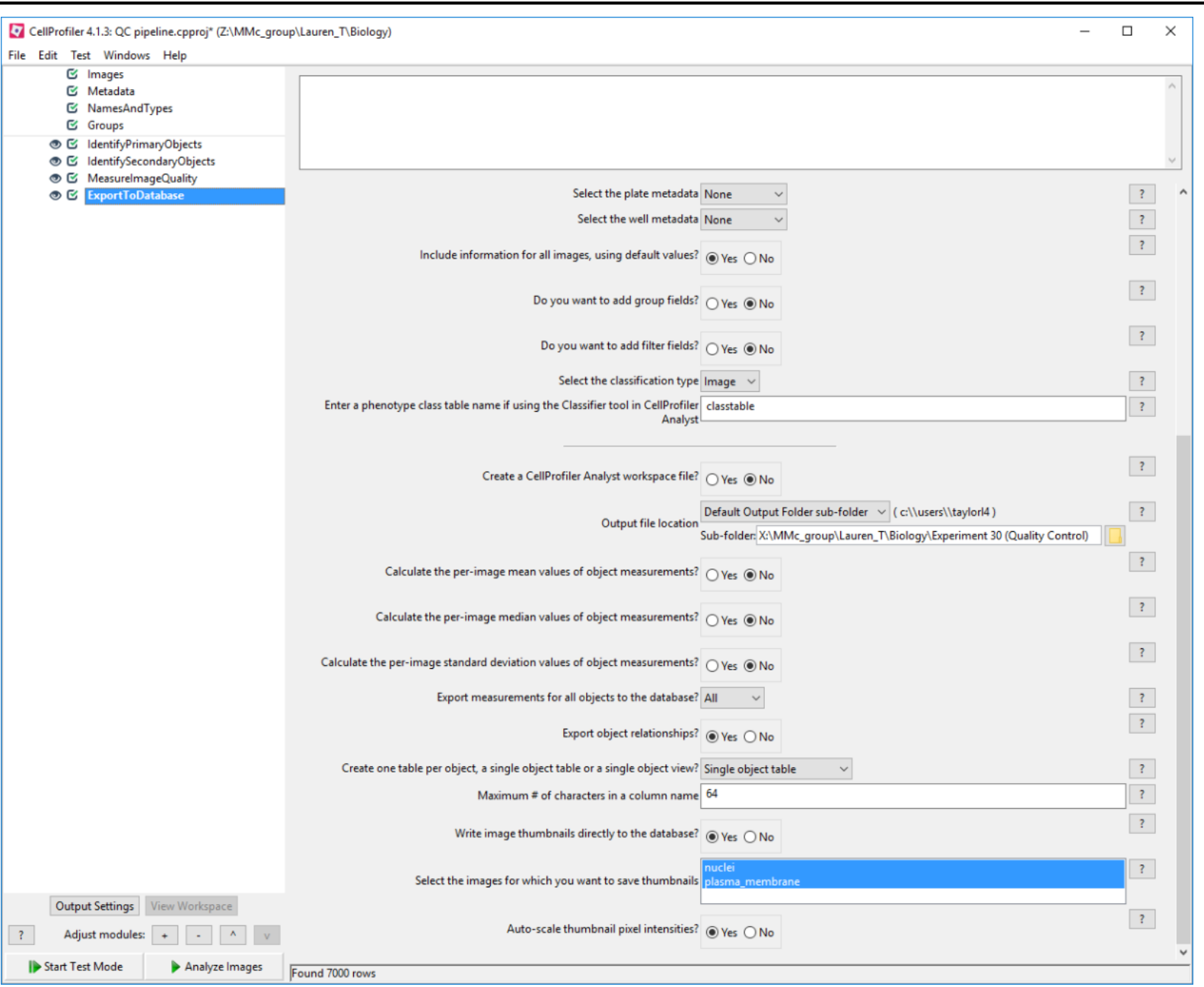

Appendix 7. CellProfiler module parameters for quality control pipeline. 


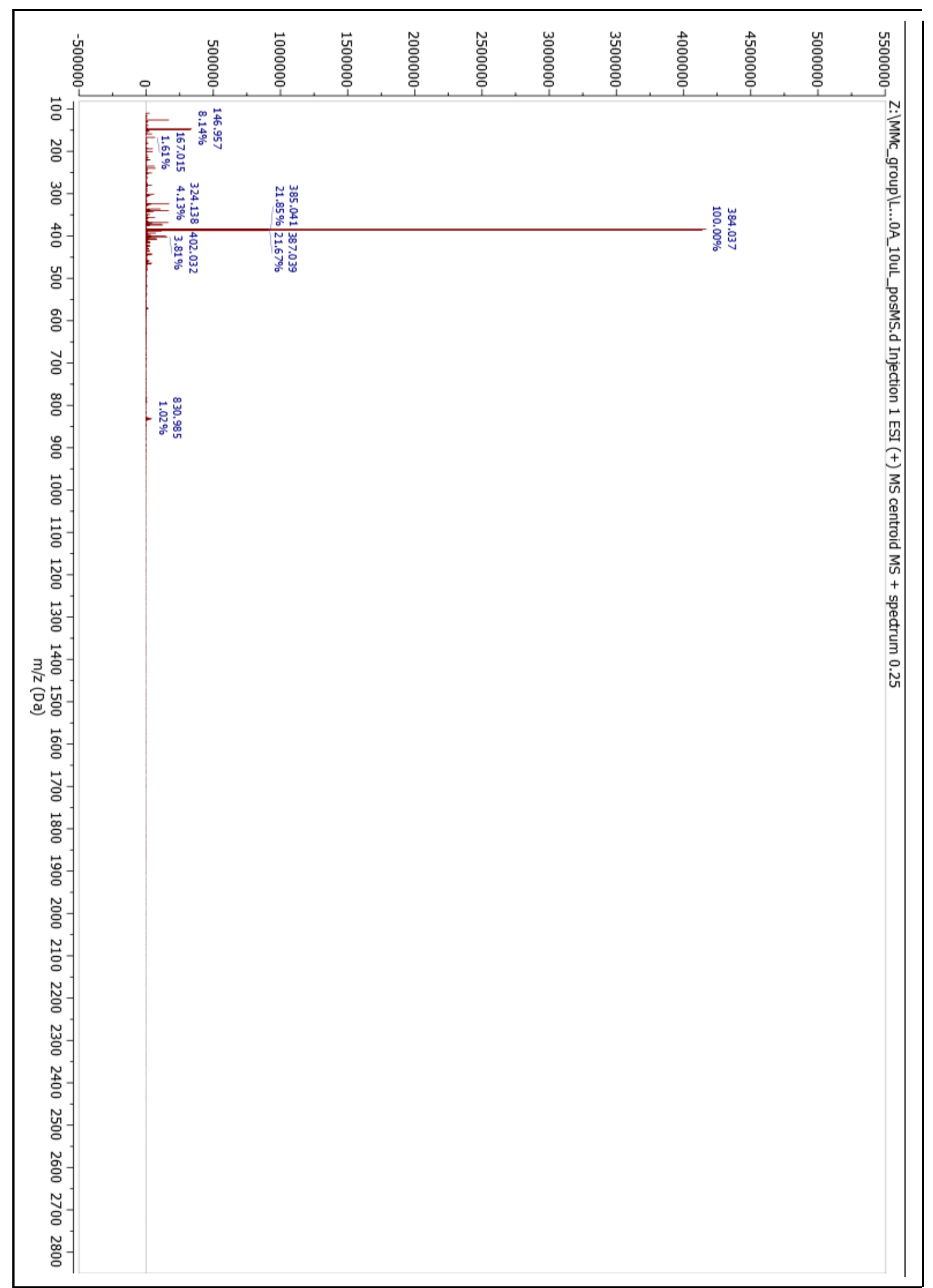

Appendix 8. Positive ion mass spectrometry data - Fraction 70A. 


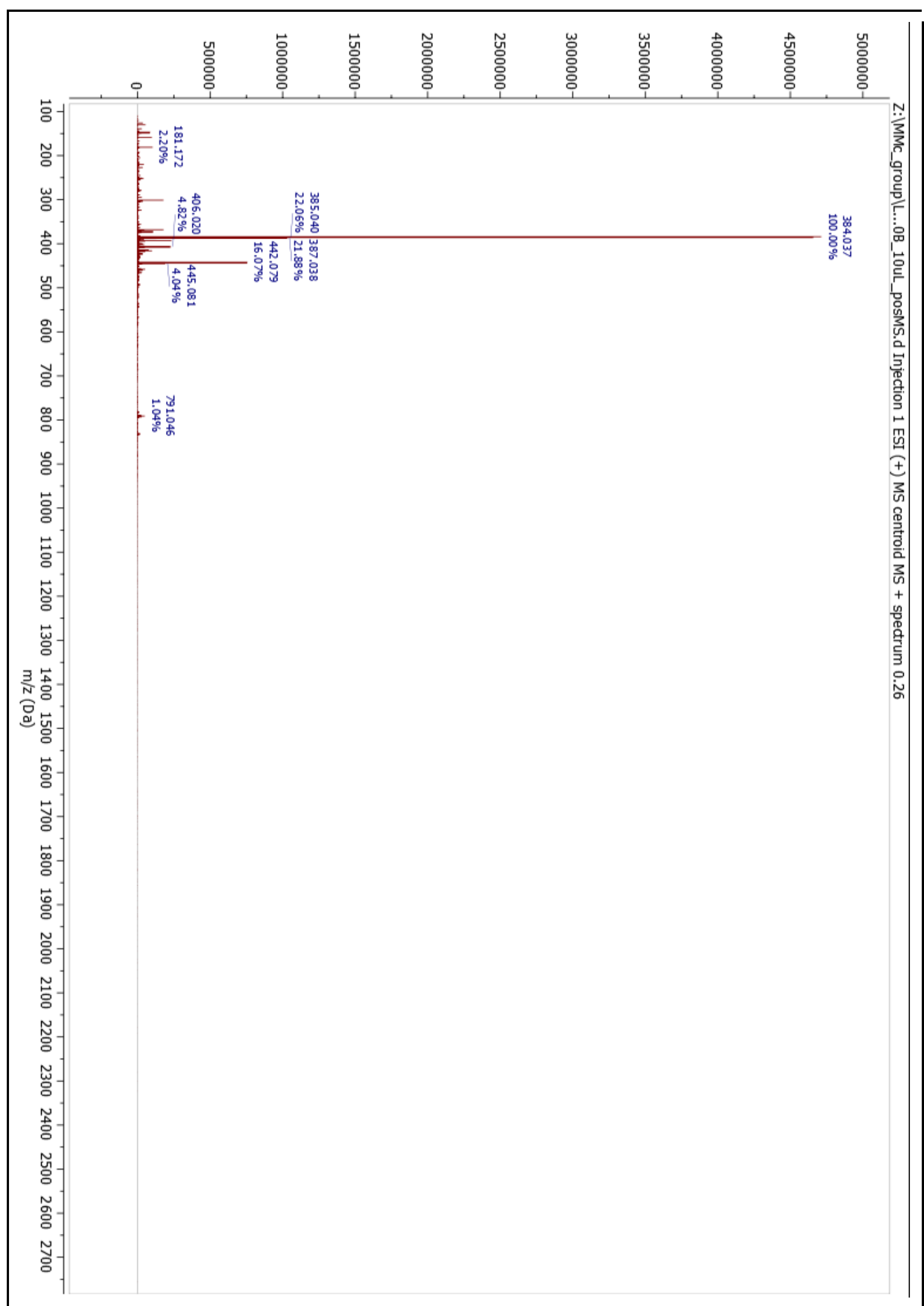

Appendix 9. Positive ion mass spectrometry data - Fraction 70B. 


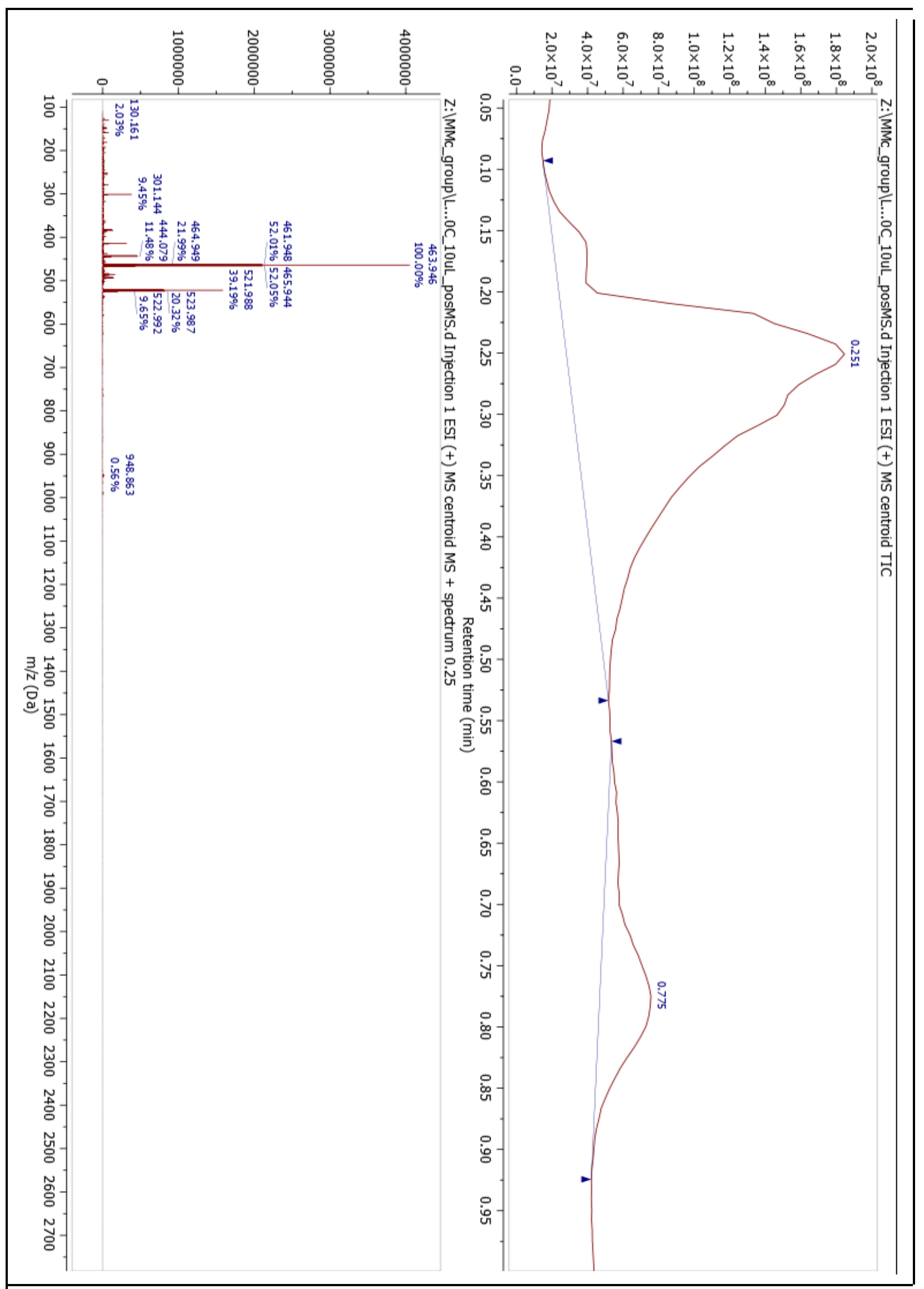

Appendix 10. Positive ion mass spectrometry data - Fraction $70 C$. 


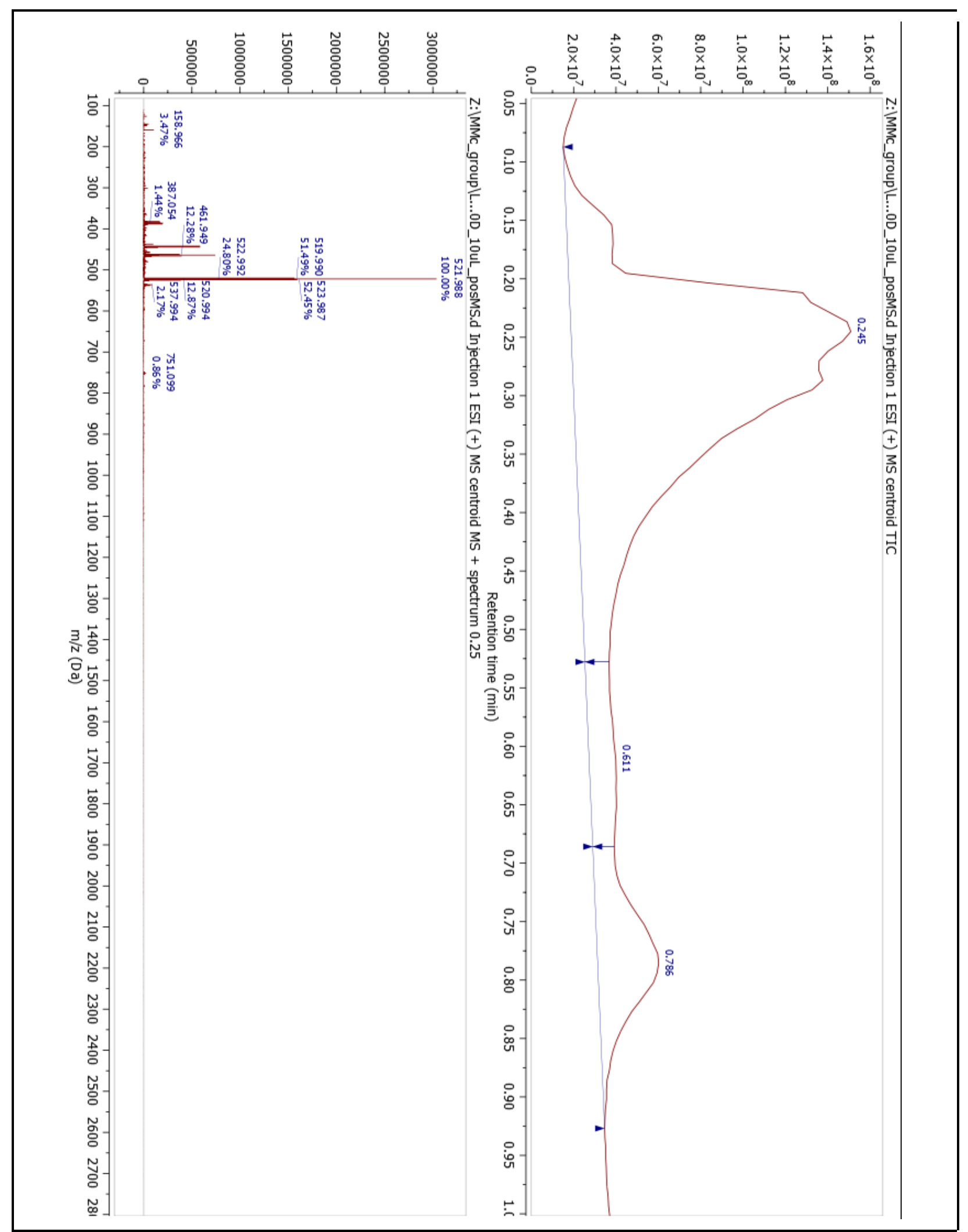

Appendix 11. Positive ion mass spectrometry data - Fraction 70D. 


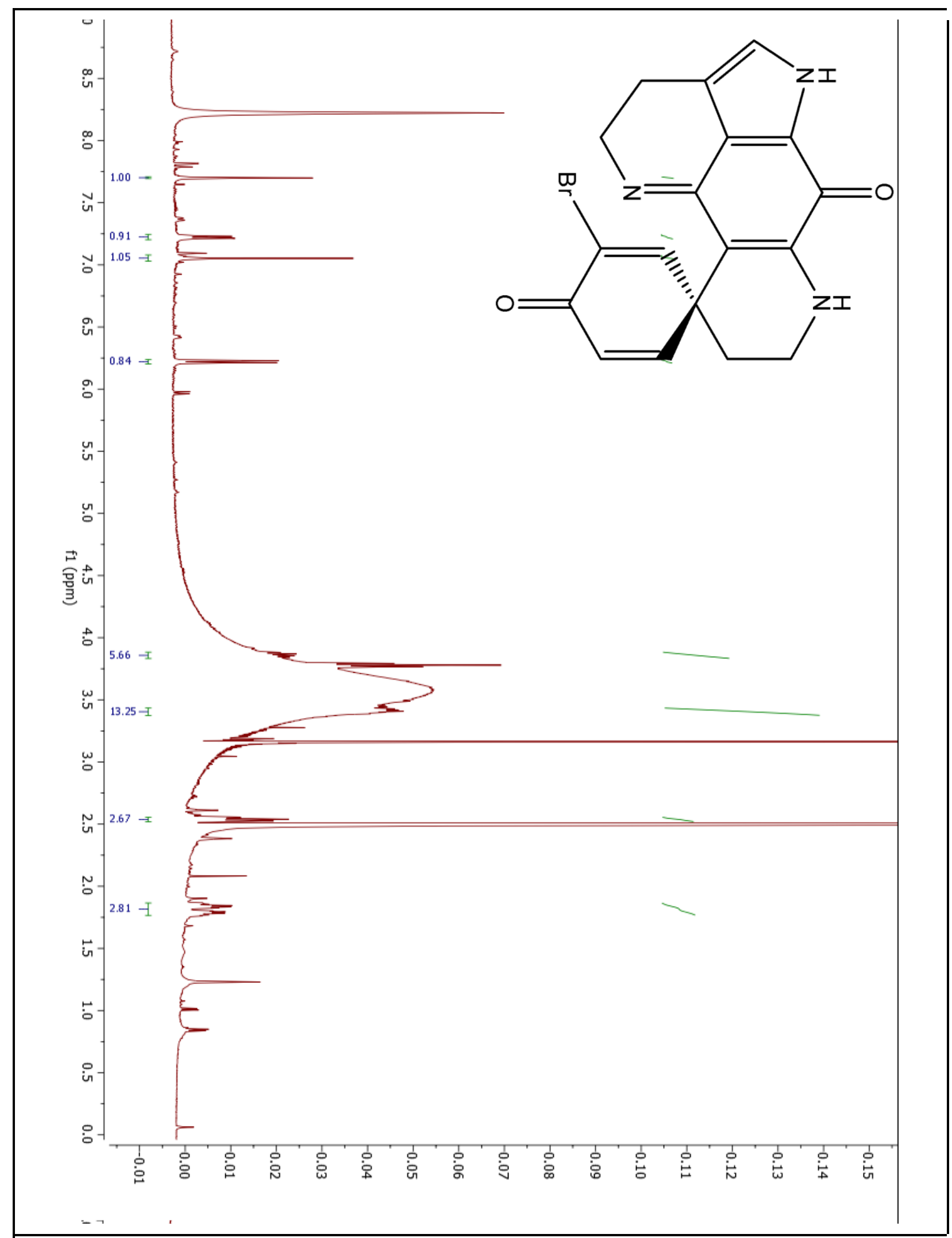

Appendix 12. ${ }^{\text {H } H}$ NMR of impure discorhabdin $E$. 


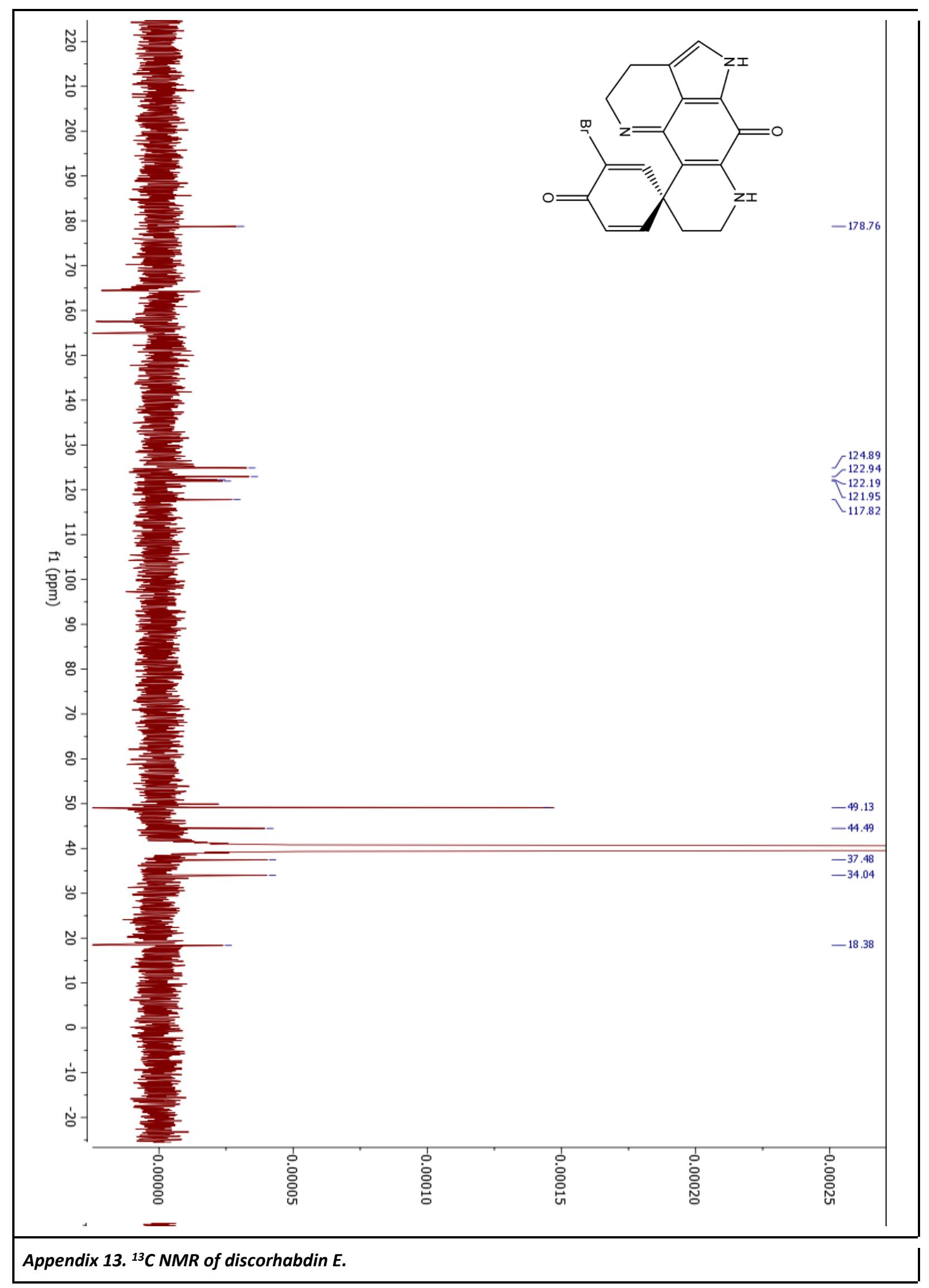




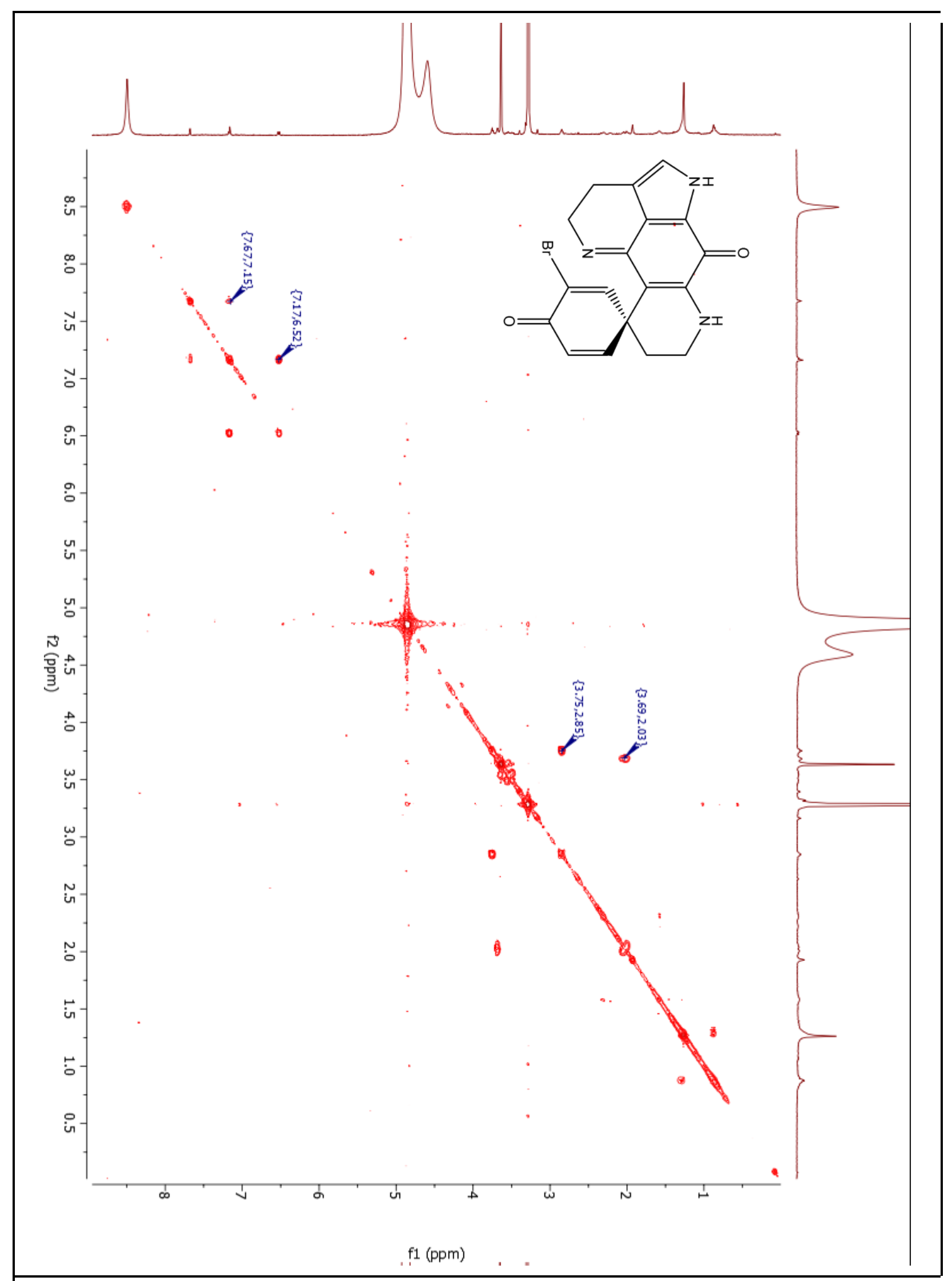

Appendix 14. COSY spectrum of discorhabdin E. 


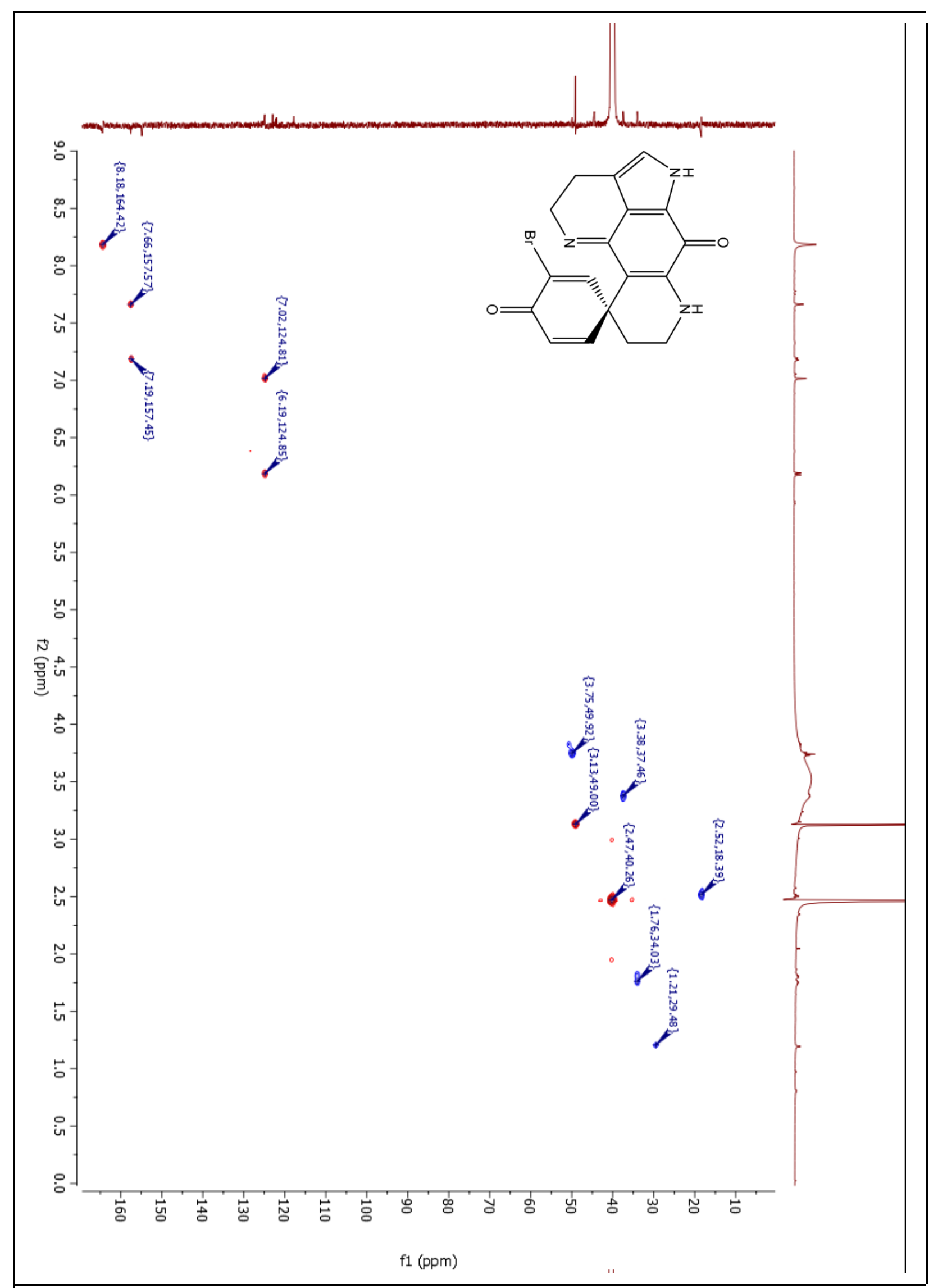

Appendix 15. HSQC spectrum of discorhabdin E. 


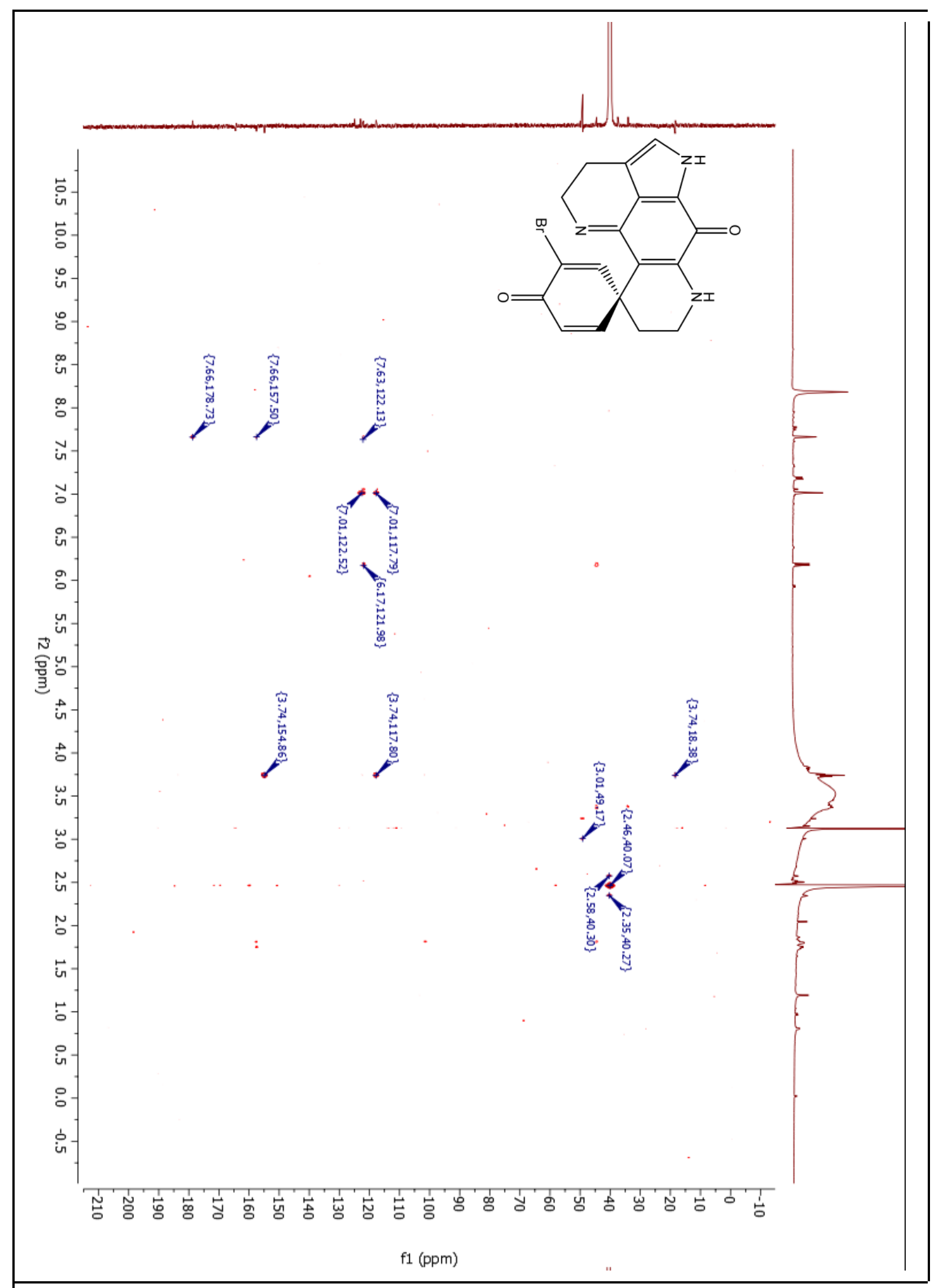

Appendix 16. HMBC spectrum of discorhabdin E. 


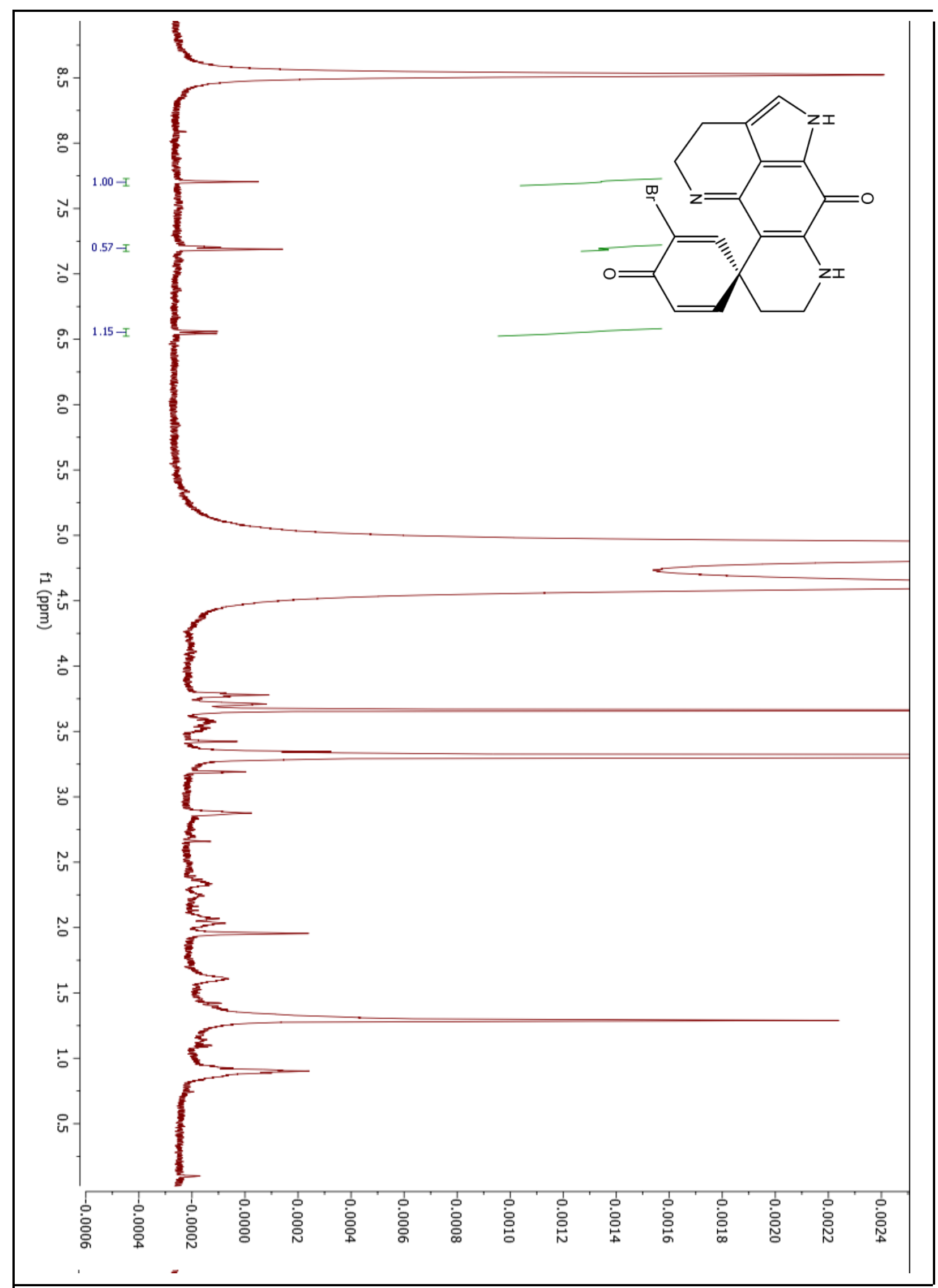

Appendix 17. ${ }^{1} \mathrm{H}$ NMR spectrum of pure discorhabdin $E$. 


\section{References}

1. Mohs, R. C. \& Greig, N. H. Drug discovery and development: Role of basic biological research. Alzheimer's Dement. (New York, N. Y.) 3, 651-657 (2017).

2. Takebe, T., Imai, R. \& Ono, S. The Current Status of Drug Discovery and Development as Originated in United States Academia: The Influence of Industrial and Academic Collaboration on Drug Discovery and Development. Clin. Transl. Sci. 11, 597-606 (2018).

3. Paul, S. M. et al. How to improve R\&D productivity: the pharmaceutical industry's grand challenge. Nature reviews. Drug discovery 9, 203-214 (2010).

4. Munos, B. Lessons from 60 years of pharmaceutical innovation. Nature reviews. Drug discovery 8, 959-968 (2009).

5. Arrowsmith, J. Phase II failures: 2008-2010. Nat. Rev. Drug Discov. 10, 328-329 (2011).

6. Arrowsmith, J. Phase III and submission failures: 2007-2010. Nat. Rev. Drug Discov. 10, 87 (2011).

7. Hughes, J., Rees, S., Kalindjian, S. \& Philpott, K. Principles of early drug discovery. Br. J. Pharmacol. 162, 1239-1249 (2011).

8. Newman, D. J. \& Cragg, G. M. Natural Products as Sources of New Drugs over the Nearly Four Decades from 01/1981 to 09/2019. J. Nat. Prod. 83, 770-803 (2020).

9. Stratton, C. F., Newman, D. J. \& Tan, D. S. Cheminformatic comparison of approved drugs from natural product versus synthetic origins. Bioorg. Med. Chem. Lett. 25, 4802-4807 (2015).

10. Zhang, Q.-W., Lin, L.-G. \& Ye, W.-C. Techniques for extraction and isolation of natural products: a comprehensive review. Chin. Med. 13, 20 (2018).

11. Blunt, J. W. et al. Marine natural products. Nat. Prod. Rep. 35, 8-53 (2018).

12. Sehgal, S. N., Baker, H. \& Vézina, C. Rapamycin (AY-22,989), a new antifungal antibiotic. II. Fermentation, isolation and characterization. J. Antibiot. (Tokyo). 28, 727-732 (1975).

13. Dutta, S., Basak, B., Bhunia, B., Chakraborty, S. \& Dey, A. Kinetics of rapamycin production by Streptomyces hygroscopicus MTCC 4003. 3 Biotech 4, 523-531 (2014).

14. Zou, X. \& Li, J. Precursor engineering and cell physiological regulation for high level rapamycin production by Streptomyces hygroscopicus. Ann. Microbiol. 63, 1371-1378 (2013).

15. Childs, B. G. et al. Senescent cells: an emerging target for diseases of ageing. Nat. Rev. Drug Discov. 16, 718-735 (2017).

16. Van Meter, M., Seluanov, A. \& Gorbunova, V. Forever young? Exploring the link between rapamycin, longevity and cancer. Cell Cycle 11, 4296-4297 (2012). 
17. Bracegirdle, J. et al. Hydrated Rubrolides from the New Zealand Tunicate Synoicum kuranui. J. Nat. Prod. 84, 544-547 (2021).

18. Bracegirdle, J., Gordon, D. P., Harvey, J. E. \& Keyzers, R. A. Kinase-Inhibitory Nucleoside Derivatives from the Pacific Bryozoan Nelliella nelliiformis. J. Nat. Prod. 83, 547-551 (2020).

19. Bracegirdle, J., Stevenson, L. J., Page, M. J., Owen, J. G. \& Keyzers, R. A. Targeted Isolation of Rubrolides from the New Zealand Marine Tunicate Synoicum kuranui. Mar. Drugs 18, (2020).

20. Bracegirdle, J. \& Keyzers, R. A. Marine-derived Polyaromatic Butenolides - Isolation, Synthesis and Biological Evaluations. Curr. Pharm. Des. 26, 4351-4361 (2020).

21. Woolner, V. H. et al. Polyhalogenated Indoles from the Red Alga Rhodophyllis membranacea: The First Isolation of Bromo-Chloro-Iodo Secondary Metabolites. $J$. Nat. Prod. 79, 463-469 (2016).

22. Woolner, V. H. et al. Halogenated Meroditerpenoids from a South Pacific Collection of the Red Alga Callophycus serratus. J. Nat. Prod. 81, 2446-2454 (2018).

23. Taufa, T. et al. Pyrroloquinoline derivatives from a Tongan specimen of the marine sponge Strongylodesma tongaensis. Tetrahedron Lett. 60, 1825-1829 (2019).

24. Taufa, T. et al. Zampanolides B-E from the Marine Sponge Cacospongia mycofijiensis: Potent Cytotoxic Macrolides with Microtubule-Stabilizing Activity. $J$. Nat. Prod. 81, 2539-2544 (2018).

25. Taufa, T., Subramani, R., Northcote, P. T. \& Keyzers, R. A. Natural Products from Tongan Marine Organisms. Molecules 26, (2021).

26. Thorpe, S. A. Oceanography. (CRC Press, 1996).

27. Sandwell, D. T., Müller, R. D., Smith, W. H. F., Garcia, E. \& Francis, R. New global marine gravity model from CryoSat-2 and Jason-1 reveals buried tectonic structure. Science (80-. ). 346, 65-67 (2014).

28. Altmann, K.-H. Drugs from the Oceans: Marine Natural Products as Leads for Drug Discovery. Chim. Int. J. Chem. 71, 646-652 (2017).

29. Carroll, A. R., Copp, B. R., Davis, R. A., Keyzers, R. A. \& Prinsep, M. R. Marine natural products. Nat. Prod. Rep. 38, 362-413 (2021).

30. Han, B.-N. et al. Natural Products from Sponges. in Symbiotic Microbiomes of Coral Reefs Sponges and Corals 329-463 (Springer Netherlands, 2019). doi:10.1007/978-94024-1612-1_15

31. Bergquist, P. Sponges. (University of California Press, 1978).

32. Proksch, P. Defensive roles for secondary metabolites from marine sponges and sponge-feeding nudibranchs. Toxicon 32, 639-655 (1994).

33. El Sayed, K. A., Youssef, D. T. A. \& Marchetti, D. Bioactive natural and semisynthetic latrunculins. J. Nat. Prod. 69, 219-223 (2006). 
34. Li, F., Peifer, C., Janussen, D. \& Tasdemir, D. New Discorhabdin Alkaloids from the Antarctic Deep-Sea Sponge Latrunculia biformis. Mar. Drugs 17, (2019).

35. Li, F., Janussen, D., Peifer, C., Pérez-Victoria, I. \& Tasdemir, D. Targeted Isolation of Tsitsikammamines from the Antarctic Deep-Sea Sponge Latrunculia biformis by Molecular Networking and Anticancer Activity. Marine Drugs 16, (2018).

36. Woolner, V. H. The Isolation and Structure Elucidation of Secondary Metabolites from Tongan Marine Invertebrates. (Victoria University of Wellington, Te Herenga Waka, 2012).

37. Keyzers, R. A., Northcote, P. T. \& Berridge, M. V. Clathriol B, a New 14\&\#946; Marine Sterol from the New Zealand Sponge Clathria lissosclera. Aust. J. Chem. 56, 279-282 (2003).

38. Lee, Y.-K., Lee, J.-H. \& Lee, H.-K. Microbial symbiosis in marine sponges. J. Microbiol. 39, 254-264 (2001).

39. Perry, N. B., Blunt, J. W. \& Munro, M. H. G. Cytotoxic pigments from new zealand sponges of the genus latrunculia : discorhabdins a, b and c. Tetrahedron 44, 17271734 (1988).

40. Yang, A., Baker, B. J., Grimwade, J., Leonard, A. \& McClintock, J. B. Discorhabdin Alkaloids from the Antarctic Sponge Latrunculia apicalis. J. Nat. Prod. 58, 1596-1599 (1995).

41. Nèeman, I., Fishelson, L. \& Kashman, Y. Isolation of a new toxin from the sponge Latrunculia magnifica in the Gulf of Aquaba (Red Sea). Mar. Biol. 30, 293-296 (1975).

42. Kashman, Y., Groweiss, A. \& Shmueli, U. Latrunculin, a new 2-thiazolidinone macrolide from the marine sponge latrunculia magnifica. Tetrahedron Lett. 21, 36293632 (1980).

43. Coué, M., Brenner, S. L., Spector, I. \& Korn, E. D. Inhibition of actin polymerization by latrunculin A. FEBS Lett. 213, 316-318 (1987).

44. Sayed, K. A. El et al. Latrunculin A and its C-17-O-carbamates inhibit prostate tumor cell invasion and HIF-1 activation in breast tumor cells. J. Nat. Prod. 71, 396-402 (2008).

45. Harayama, Y. \& Kita, Y. Pyrroloiminoquinone Alkaloids: Discorhabdins and Makaluvamines. Curr. Org. Chem. 9, 1567-1588 (2005).

46. Alvarez, B., Bergquist, P. R. \& Battershill, C. N. Taxonomic revision of the genus Latrunculia Du Bocage (Porifera: Demospongiae: Latrunculiidae) in New Zealand. New Zeal. J. Mar. Freshw. Res. 36, 151-184 (2002).

47. Copp, B. R., Fulton, K. F., Perry, N. B., Blunt, J. W. \& Munro, M. H. G. Natural and Synthetic Derivatives of Discorhabdin C, a Cytotoxic Pigment from the New Zealand Sponge Latrunculia cf. bocagei. J. Org. Chem. 59, 8233-8238 (1994).

48. Hu, J. F., Fan, H., Xiong, J. \& Wu, S. B. Discorhabdins and pyrroloiminoquinonerelated alkaloids. Chem. Rev. 111, 5465-5491 (2011). 
49. Willis, C., Nyffeler, J. \& Harrill, J. Phenotypic Profiling of Reference Chemicals across Biologically Diverse Cell Types Using the Cell Painting Assay. SLAS Discov. 25, 755-769 (2020).

50. Carpenter, A. E. et al. CellProfiler: image analysis software for identifying and quantifying cell phenotypes. Genome Biol. 7, R100 (2006).

51. Ljosa, V. et al. Comparison of Methods for Image-Based Profiling of Cellular Morphological Responses to Small-Molecule Treatment. J. Biomol. Screen. 18, 13211329 (2013).

52. Futamura, Y. et al. Morphobase, an Encyclopedic Cell Morphology Database, and Its Use for Drug Target Identification. Chem. Biol. 19, 1620-1630 (2012).

53. Rämö, P., Sacher, R., Snijder, B., Begemann, B. \& Pelkmans, L. CellClassifier: supervised learning of cellular phenotypes. Bioinformatics 25, 3028-3030 (2009).

54. Sumiya, E. et al. Cell-Morphology Profiling of a Natural Product Library Identifies Bisebromoamide and Miuraenamide A as Actin Filament Stabilizers. ACS Chem. Biol. 6, 425-431 (2011).

55. Loo, L.-H., Wu, L. F. \& Altschuler, S. J. Image-based multivariate profiling of drug responses from single cells. Nat. Methods 4, 445-453 (2007).

56. McQuin, C. et al. CellProfiler 3.0: Next-generation image processing for biology. PLOS Biol. 16, e2005970 (2018).

57. Bray, M.-A. \& Carpenter, A. E. Quality Control for High-Throughput Imaging Experiments Using Machine Learning in Cellprofiler. Methods Mol. Biol. 1683, 89112 (2018).

58. Lamprecht, M. R., Sabatini, D. M. \& Carpenter, A. E. CellProfiler ${ }^{\text {TM}}$ : free, versatile software for automated biological image analysis. Biotechniques 42, 71-75 (2007).

59. Institute, B. CellProfiler Tutorials. (2021). Available at: https://cellprofiler.org/tutorials.

60. Bray, M.-A. et al. Cell Painting, a high-content image-based assay for morphological profiling using multiplexed fluorescent dyes. Nat. Protoc. 11, 1757-1774 (2016).

61. Svenningsen, E. B. \& Poulsen, T. B. Establishing cell painting in a smaller chemical biology lab - A report from the frontier. Bioorg. Med. Chem. 27, 2609-2615 (2019).

62. Gustafsdottir, S. M. et al. Multiplex Cytological Profiling Assay to Measure Diverse Cellular States. PLoS One 8, e80999 (2013).

63. West, L. M. The Isolation of Secondary Metabolites from New Zealand Marine Sponges. (Victoria University of Wellington, 2001).

64. Chen, Q.-H. \& Kingston, D. G. I. Zampanolide and dactylolide: cytotoxic tubulinassembly agents and promising anticancer leads. Nat. Prod. Rep. 31, 1202-1226 (2014).

65. Miller, J. H., Singh, A. J. \& Northcote, P. T. Microtubule-Stabilizing Drugs from 
Marine Sponges: Focus on Peloruside A and Zampanolide. Mar. Drugs 8, 1059-1079 (2010).

66. Field, J. J. et al. Microtubule-Stabilizing Activity of Zampanolide, a Potent Macrolide Isolated from the Tongan Marine Sponge Cacospongia mycofijiensis. J. Med. Chem. 52, 7328-7332 (2009).

67. Field, J. et al. Zampanolide, a Microtubule-Stabilizing Agent, Is Active in Resistant Cancer Cells and Inhibits Cell Migration. Int. J. Mol. Sci. 18, 971 (2017).

68. Zurwerra, D. et al. Total Synthesis of (-)-Zampanolide and Structure-Activity Relationship Studies on (-)-Dactylolide Derivatives. Chem. - A Eur. J. 18, 1686816883 (2012).

69. Gupta, M. L., Bode, C. J., Georg, G. I. \& Himes, R. H. Understanding tubulin-Taxol interactions: Mutations that impart Taxol binding to yeast tubulin. Proc. Natl. Acad. Sci. 100, 6394-6397 (2003).

70. Wall, M. E. \& Wani, M. C. Camptothecin and taxol: from discovery to clinic. $J$. Ethnopharmacol. 51, 239-254 (1996).

71. Wani, M. C., Taylor, H. L., Wall, M. E., Coggon, P. \& McPhail, A. T. Plant antitumor agents. VI. Isolation and structure of taxol, a novel antileukemic and antitumor agent from Taxus brevifolia. J. Am. Chem. Soc. 93, 2325-2327 (1971).

72. Wall, M. E. \& Wani, M. C. Paclitaxel: From Discovery to Clinic. in 18-30 (1994). doi:10.1021/bk-1995-0583.ch002

73. Kellogg, E. H. et al. Insights into the Distinct Mechanisms of Action of Taxane and Non-Taxane Microtubule Stabilizers from Cryo-EM Structures. J. Mol. Biol. 429, 633-646 (2017).

74. Pommier, Y., Leo, E., Zhang, H. \& Marchand, C. DNA Topoisomerases and Their Poisoning by Anticancer and Antibacterial Drugs. Chem. Biol. 17, 421-433 (2010).

75. David Foglesong, P., Reckord, C. \& Swink, S. Doxorubicin inhibits human DNA topoisomerase I. Cancer Chemother. Pharmacol. 30, 123-125 (1992).

76. Punia, R., Raina, K., Agarwal, R. \& Singh, R. P. Acacetin enhances the therapeutic efficacy of doxorubicin in non-small-cell lung carcinoma cells. PLoS One 12, e0182870 (2017).

77. Denny, B. J., Wheelhouse, R. T., Stevens, M. F. G., Tsang, L. L. H. \& Slack, J. A. NMR and Molecular Modeling Investigation of the Mechanism of Activation of the Antitumor Drug Temozolomide and Its Interaction with DNA. Biochemistry 33, 90459051 (1994).

78. Fabre, M.-S. et al. The oncogene BCL6 is up-regulated in glioblastoma in response to DNA damage, and drives survival after therapy. PLoS One 15, e0231470 (2020).

79. Xin, P. et al. Efficacy of the dual PI3K and mTOR inhibitor NVP-BEZ235 in combination with imatinib mesylate against chronic myelogenous leukemia cell lines. Drug Des. Devel. Ther. Volume11, 1115-1126 (2017). 
80. Shi, F. et al. The dual PI3K/mTOR inhibitor dactolisib elicits anti-tumor activity in vitro and in vivo. Oncotarget $\mathbf{9},(2018)$.

81. Uitdehaag, J. C. M. et al. Cell Panel Profiling Reveals Conserved Therapeutic Clusters and Differentiates the Mechanism of Action of Different PI3K/mTOR, Aurora Kinase and EZH2 Inhibitors. Mol. Cancer Ther. 15, 3097-3109 (2016).

82. Xia, A., Li, H., Li, R., Lu, L. \& Wu, X. Co-treatment with BEZ235 enhances chemosensitivity of A549/DDP cells to cisplatin via inhibition of PI3K/Akt/mTOR signaling and downregulation of ERCC1 expression. Oncol. Rep. (2018). doi:10.3892/or.2018.6583

83. Stamatkin, C., Ratermann, K. L., Overley, C. W. \& Black, E. P. Inhibition of class IA PI3K enzymes in non-small cell lung cancer cells uncovers functional compensation among isoforms. Cancer Biol. Ther. 16, 1341-1352 (2015).

84. TONG, N. et al. Berberine sensitizes mutliple human cancer cells to the anticancer effects of doxorubicin in vitro. Oncol. Lett. 3, 1263-1267 (2012).

85. Wang, J. Studies on the Synthesis of Marine Natural Product (-)-Zampanolide and its Analogues. (Te Herenga Waka - Victoria University of Wellington, 2016).

86. He, S. et al. Comparison of active and passive targeting of doxorubicin for somatostatin receptor 2 positive tumor models by octreotide-modified HPMA copolymer-doxorubicin conjugates. Drug Deliv. 23, 285-296 (2016).

87. Jiang, L., Li, X., Liu, L. \& Zhang, Q. Thiolated chitosan-modified PLA-PCL-TPGS nanoparticles for oral chemotherapy of lung cancer. Nanoscale Res. Lett. 8, 66 (2013).

88. ACS Publications Journal of Natural Products Author Guidelines.

89. Li, J. et al. Involvement of Cdc $25 \mathrm{c}$ in Cell Cycle Alteration of a Radioresistant Lung Cancer Cell Line Established with Fractionated Ionizing Radiation. Asian Pacific J. Cancer Prev. 14, 5725-5730 (2013).

90. Field, J. J. et al. Zampanolide, a Potent New Microtubule-Stabilizing Agent, Covalently Reacts with the Taxane Luminal Site in Tubulin $\alpha, \beta$-Heterodimers and Microtubules. Chem. Biol. 19, 686-698 (2012).

91. Mikhailov, A., Shinohara, M. \& Rieder, C. L. Topoisomerase II and histone deacetylase inhibitors delay the G2/M transition by triggering the p38 MAPK checkpoint pathway. J. Cell Biol. 166, 517-526 (2004).

92. D`Arcy, N. \& Gabrielli, B. Topoisomerase II Inhibitors and Poisons, and the Influence of Cell Cycle Checkpoints. Curr. Med. Chem. 24, (2017).

93. Shi, F. et al. The dual PI3K/mTOR inhibitor dactolisib elicits anti-tumor activity in vitro and in vivo. Oncotarget 9, 706-717 (2018).

94. Xu, N., Lao, Y., Zhang, Y. \& Gillespie, D. A. Akt: A Double-Edged Sword in Cell Proliferation and Genome Stability. J. Oncol. 2012, 1-15 (2012).

95. Bray, M.-A., Fraser, A. N., Hasaka, T. P. \& Carpenter, A. E. Workflow and Metrics for Image Quality Control in Large-Scale High-Content Screens. J. Biomol. Screen. 
17, 266-274 (2012).

96. Yang, A., Baker, B. J., Grimwade, J., Leonard, A. \& McClintock, J. B. Discorhabdin Alkaloids from the Antarctic Sponge Latrunculia apicalis. J. Nat. Prod. 58, 1596-1599 (1995).

97. Li, F., Janussen, D. \& Tasdemir, D. New Discorhabdin B Dimers with Anticancer Activity from the Antarctic Deep-Sea Sponge Latrunculia biformis. Marine Drugs 18, (2020).

98. Keyzers, R. A., Samaai, T. \& Davies-Coleman, M. T. Novel pyrroloquinoline ribosides from the South African latrunculid sponge Strongylodesma aliwaliensis. Tetrahedron Lett. 45, 9415-9418 (2004).

99. Gunasekera, S. P., Zuleta, I. A., Longley, R. E., Wright, A. E. \& Pomponi, S. A. Discorhabdins S, T, and U, New Cytotoxic Pyrroloiminoquinones from a Deep-Water Caribbean Sponge of the Genus Batzella. J. Nat. Prod. 66, 1615-1617 (2003).

100. Blunt, JW. ; Munro, M. MarinLit: A database of the literature on marine natural products. (2017).

101. Klionsky, D. J. et al. Guidelines for the use and interpretation of assays for monitoring autophagy. Autophagy 8, 445-544 (2012).

102. Kocaturk, N. M. et al. Autophagy as a molecular target for cancer treatment. Eur. J. Pharm. Sci. 134, 116-137 (2019).

103. Liu, B., Wen, X. \& Cheng, Y. Survival or death: disequilibrating the oncogenic and tumor suppressive autophagy in cancer. Cell Death Dis. 4, e892-e892 (2013).

104. He, C. \& Klionsky, D. J. Regulation mechanisms and signaling pathways of autophagy. Annu. Rev. Genet. 43, 67-93 (2009).

105. Kainz, K. et al. Methods to Assess Autophagy and Chronological Aging in Yeast. in 367-394 (2017). doi:10.1016/bs.mie.2016.09.086

106. Wada, Y. et al. The synthetic and biological studies of discorhabdins and related compounds. Org. Biomol. Chem. 9, 4959 (2011).

107. Goey, A. K. L. et al. Screening and Biological Effects of Marine Pyrroloiminoquinone Alkaloids: Potential Inhibitors of the HIF-1 $\alpha /$ p300 Interaction. J. Nat. Prod. 79, $1267-$ 1275 (2016).

108. Lee, S., Hallis, S. P., Jung, K.-A., Ryu, D. \& Kwak, M.-K. Impairment of HIF-1 $\alpha-$ mediated metabolic adaption by NRF2-silencing in breast cancer cells. Redox Biol. 24, 101210 (2019). 